\title{
EFFECTS OF BIO-COMPOSITES IN CORRUGATED SANDWICH PANELS UNDER EDGEWISE COMPRESSION LOADING
}

\author{
A Thesis \\ presented to \\ the Faculty of California Polytechnic State University, \\ San Luis Obispo
}

\author{
In Partial Fulfillment \\ of the Requirements for the Degree \\ Master of Science in Mechanical Engineering
}

By

Jalen C. Mano

May 2019 
(C) 2019

Jalen C. Mano

ALL RIGHTS RESERVED 


\section{COMMITTEE MEMBERSHIP}

TITLE: Effects of Bio-Composites in Corrugated Sandwich

Panels Under Edgewise Compression Loading

\section{AUTHOR: Jalen C. Mano}

DATE SUBMITTED: June 2019

COMMITTEE CHAIR: $\quad$ Eltahry Elghandour, Ph. D.

Associate Professor of Mechanical Engineering

COMMITTEE MEMBER: Mohammed Noori, Ph. D.

Professor of Mechanical Engineering

COMMITTEE MEMBER: Joseph Mello Ph. D.

Professor of Mechanical Engineering 


\begin{abstract}
Effects of Bio-Composites in Corrugated Sandwich Panels Under Edgewise Compression Loading

Jalen C. Mano
\end{abstract}

Present day composite sandwich panels provide incredible strength. Their largest problem, however, is early bonding failure between the core and the skin. This is due to the low bonding surface area of present cores like honeycomb. Corrugated structures could provide a remedy for this with their much larger bonding surface area. Corrugated structures have extreme mechanical properties deeming them particularly useful in aerospace and automotive applications. However, previous research has shown that the stiffness of carbon fiber causes debonding and drastic failure when used as both a core and a skin. Bio-composites have properties that could strengthen the corrugated sandwich panel against such debonding and increase the strength of the structure while making it cheaper and more environmentally friendly.

This thesis presents the optimum design, manufacturing, and testing of corrugated sandwich panel structures with integrated bio-composites under edgewise compression loading. To do this, optimum corrugation geometry was identified using theoretical analysis of the moment and bonding area of the shape. Control tests with carbon fiber and hemp were conducted. The bio-composite was integrated in both the core and the skin individually in corrugated sandwich panels. The cases tested were all-carbon fiber, hemp skin with carbon fiber core, carbon fiber skin with hemp core, and all-hemp. These corrugated structures were analyzed by conducting compression loading tests on varying lengths of single-ligament panels utilizing trapezoidal corrugation as the core and a flat plate as the skin. The lengths tested were 1,2, 3, and 4 inches. As many samples as possible were manufactured out of limited material with heavier focus on creating the shorter samples. The goal of this testing was, first, to determine if hemp fibers were viable as a substitute for certain sections of the traditional composite structure, and second, to see if integrating hemp fibers would solve the problems of debonding seen in the all-carbon fiber samples seen in previous research. To determine mechanical property viability, the ultimate load and stiffness were investigated for each sample, as well as investigation of the failure modes seen in the test. Secondary goals were to see at what length buckling behavior became an issue and to see if this corrugated structure and all its failure modes could be simulated in finite element analysis.

At the 1-inch and 2-inch lengths where minimal buckling was encountered, the hemp core-carbon skin samples showed better results than both the all-carbon fiber and the all-hemp samples with a $4 \%$ and $6 \%$ increase in average ultimate load and a $11 \%$ and $47 \%$ increase in stiffness, respectively. From these results, it was concluded that hybrid biocomposite structures can have comparable mechanical properties to traditional composites and can solve bonding failure. 


\section{ACKNOWLEDGMENTS}

I would first like to thank my advisor, Dr. Eltahry Elghandour, who has spent countless hours in the composites lab mentoring and guiding me through much of my composites journey, culminating in this thesis. Dr. Elghandour consistently challenged me to work harder and to deepen my understanding of not only composites, but mechanical engineering and design principles in general. In no small part to him, this thesis was made possible, in addition to the vast and diverse mechanical engineering knowledge I gained in the past several years.

I would also like to thank Dr. Mohammed Noori and Dr. Joseph Mello, my thesis committee members. They provided valuable insight and expertise in their respective areas of expertise and in general. In addition, I would like to thank Dr. Kim Shollenberger, who, as my graduate coordinator, helped me greatly with logistics and deadlines.

I would next like to thank my mom, Elena, my dad, Jim, and my sister, Audrey, who provided unbelievable patience, support, and advice throughout my entire life and especially throughout my time as a graduate student. I know they will continue to do so unconditionally, and I only hope I can do the same for them as I move into the next stage of life.

In addition, I would like to thank my internship mentor at Sandia National Labs, Corinne Hagan, a fellow Cal Poly graduate. In such a short time, she taught me an incredible amount about thesis writing, composites manufacture and testing, and career success. I would also like to thank my fellow graduate students Jacob Chess and Thomas Rohrbach, who provided camaraderie and friendship amidst challenging classes and projects. They also proved to be great examples of successful graduate students. I would also like to thank Josh DiMaggio who spent hours with me in the composites lab helping me with testing. I would also like to thank all my friends and roommates throughout college for making it such a great experience and for what I know will be a great many years of friendship to come. 


\section{TABLE OF CONTENTS}

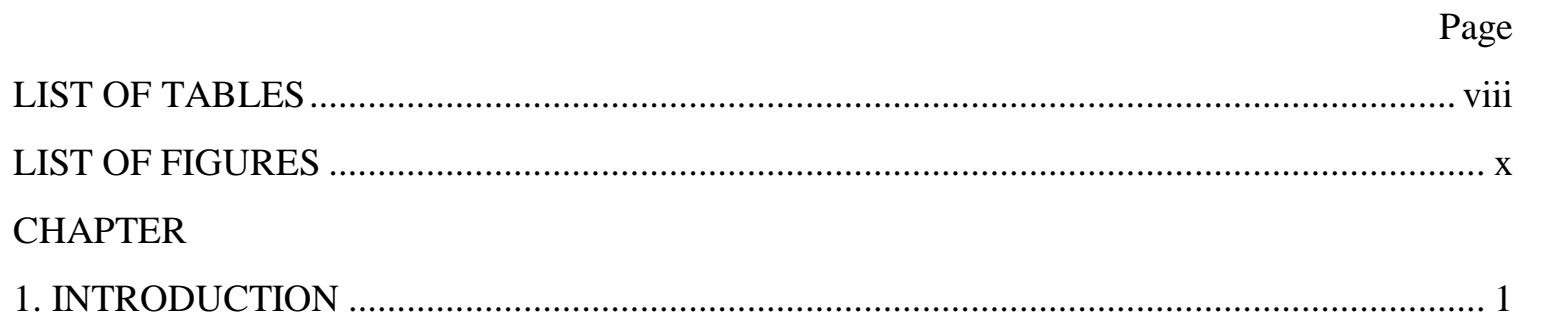

1.1 An Overview of Traditional Composites ..................................................................... 1

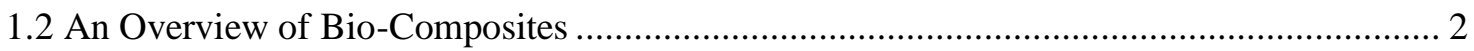

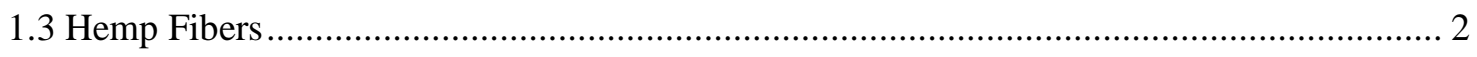

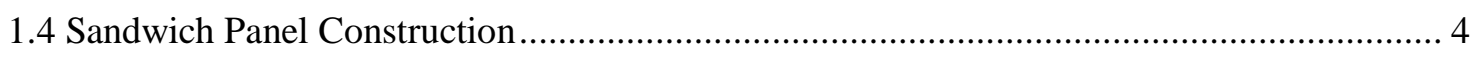

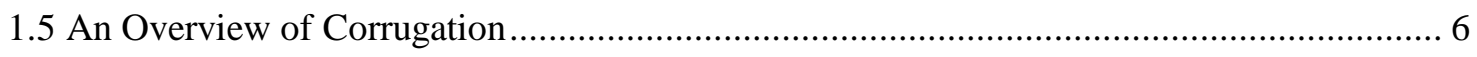

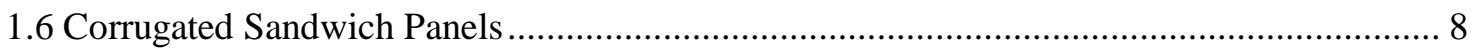

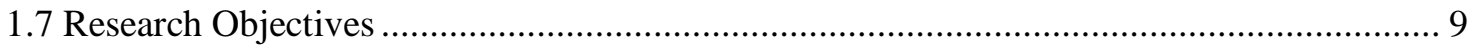

2. CORRUGATION GEOMETRY THEORETICAL ANALYSIS ........................................ 11

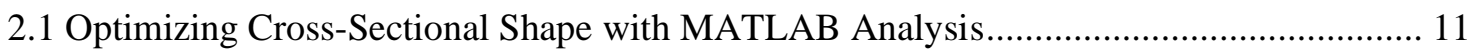

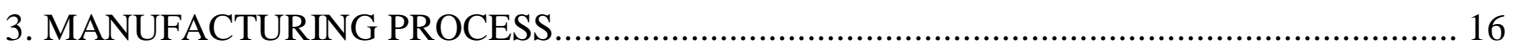

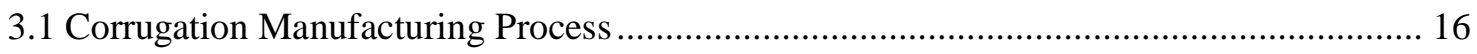

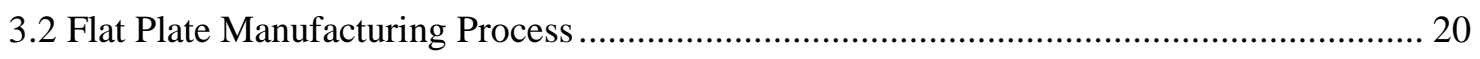

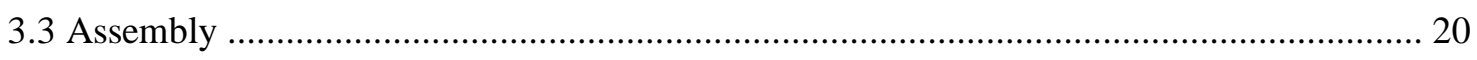

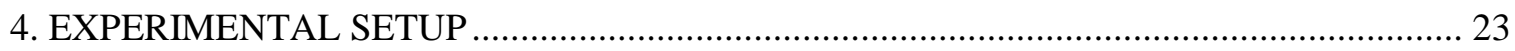

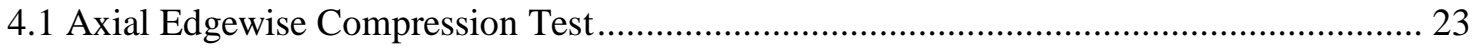

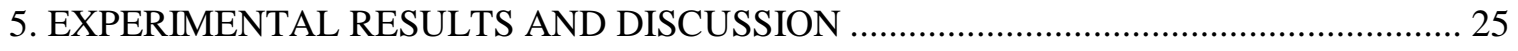

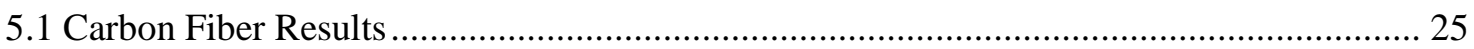

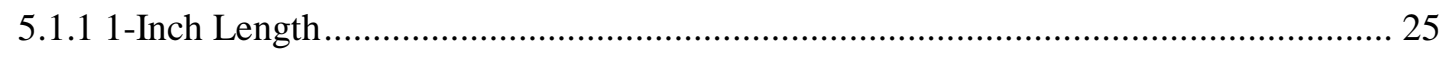

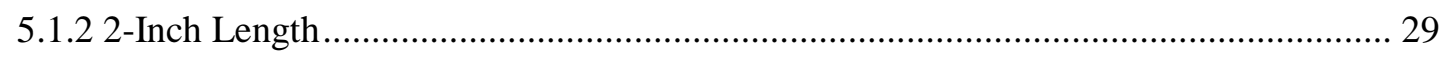

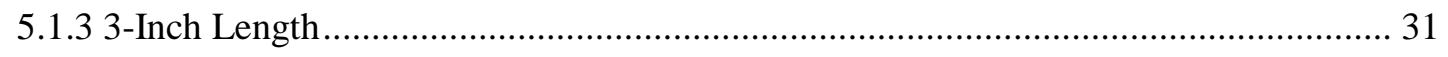

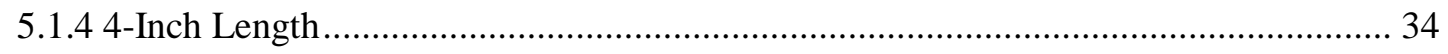

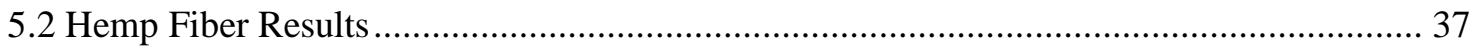

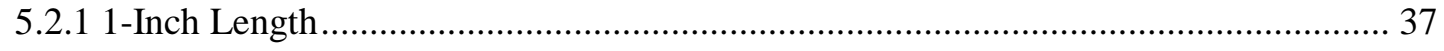

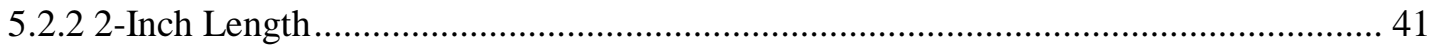

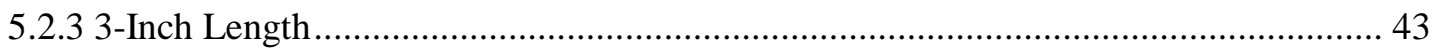

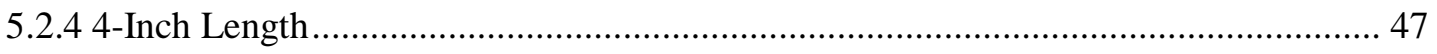




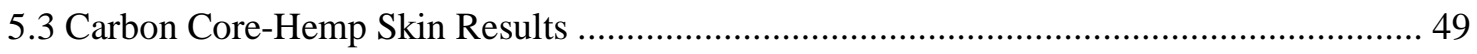

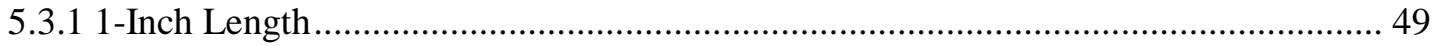

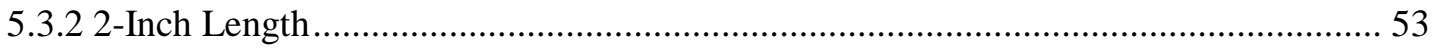

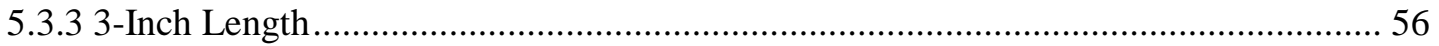

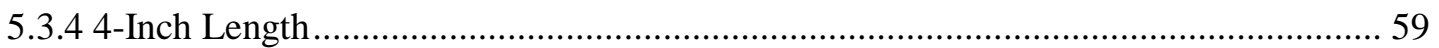

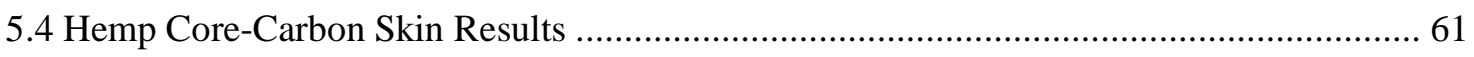

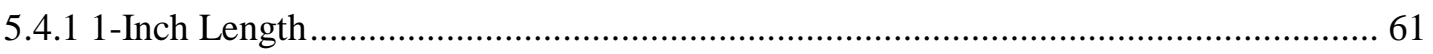

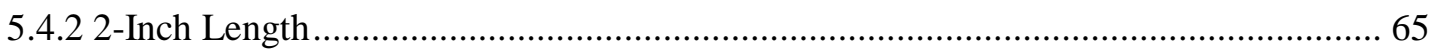

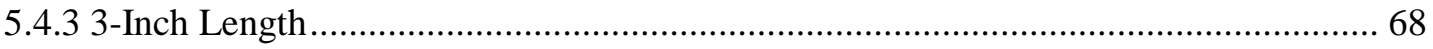

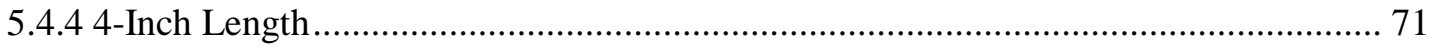

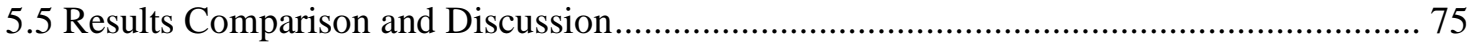

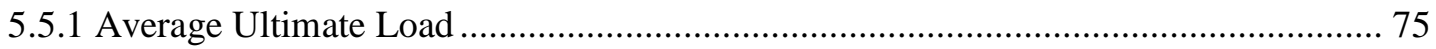

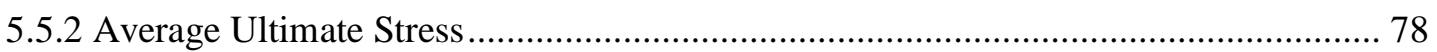

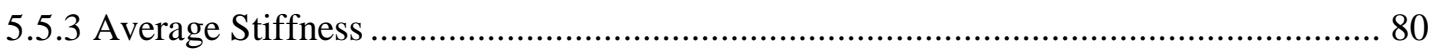

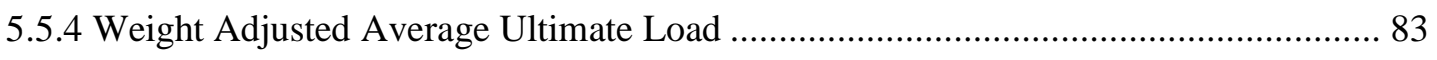

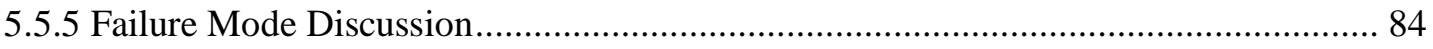

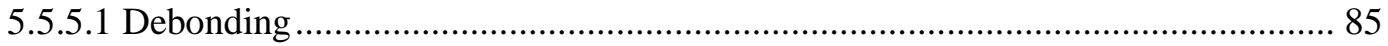

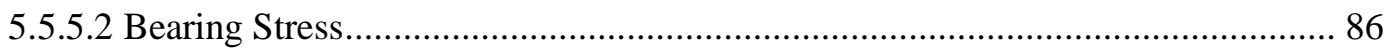

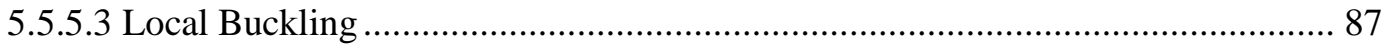

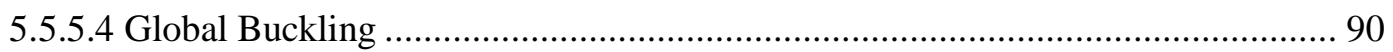

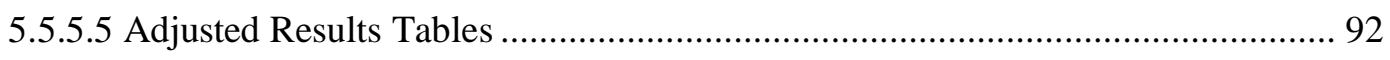

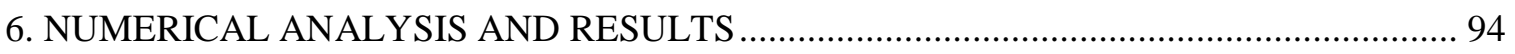

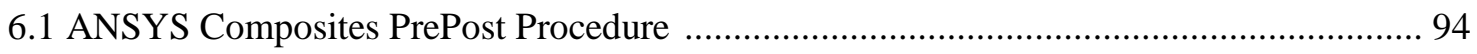

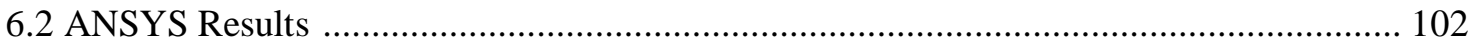

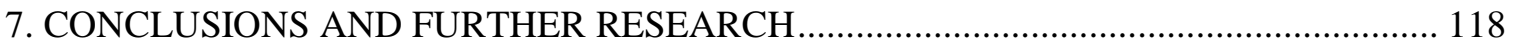

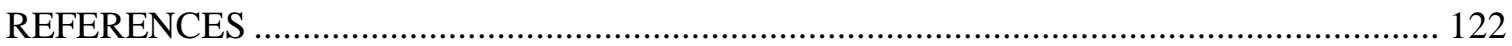




\section{LIST OF TABLES}

Table

Page

1: $\quad$ Trapezoidal Cross-Sectional Area Moment of Inertia ................................................. 13

2: $\quad$ Rectangular Cross-Sectional Area Moment of Inertia ..................................................... 13

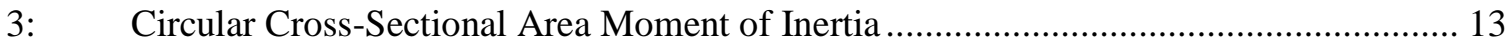

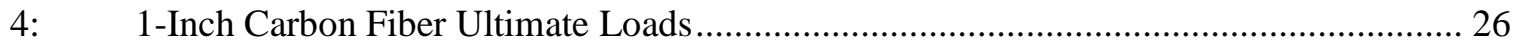

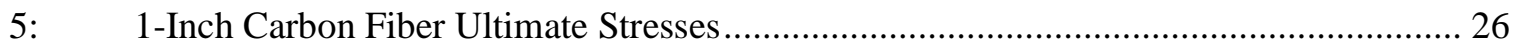

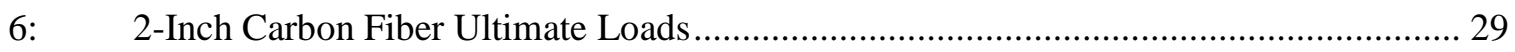

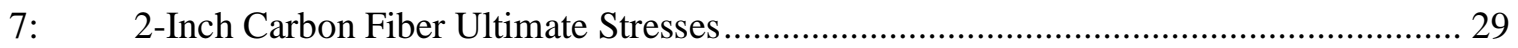

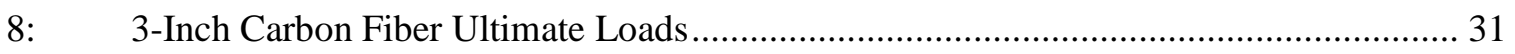

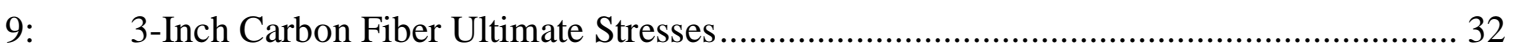

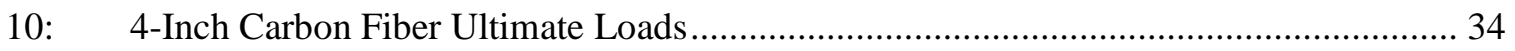

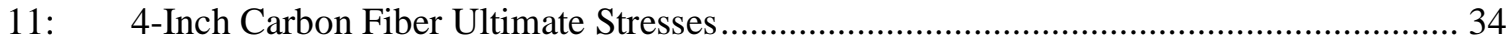

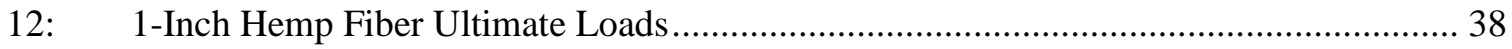

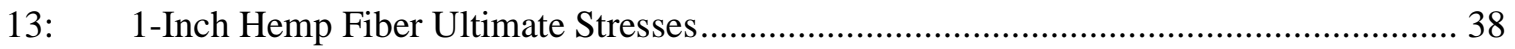

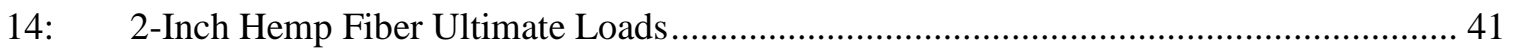

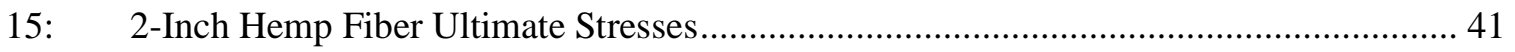

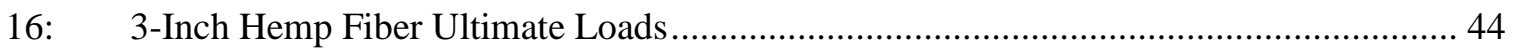

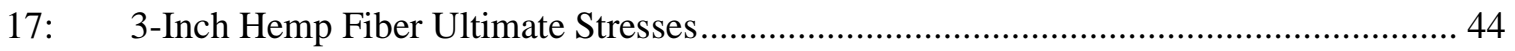

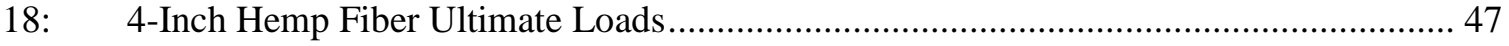

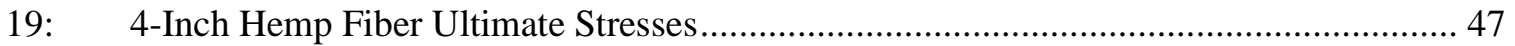

20: $\quad$ 1-Inch Carbon Core-Hemp Skin Ultimate Loads .......................................................... 50

21: 1-Inch Carbon Core-Hemp Skin Ultimate Stresses ...................................................... 50

22: $\quad$ 2-Inch Carbon Core-Hemp Skin Ultimate Loads ........................................................... 53

23: 2-Inch Carbon Core-Hemp Skin Ultimate Stresses ..................................................... 53

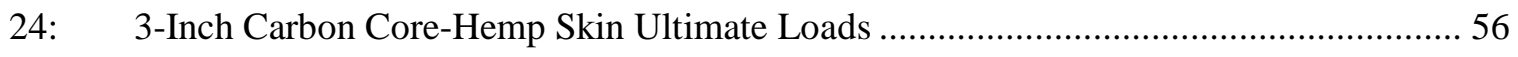

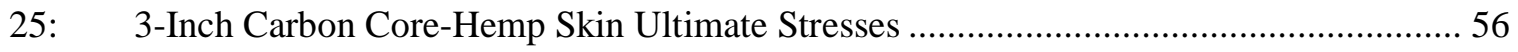

26: $\quad$ 4-Inch Carbon Core-Hemp Skin Ultimate Loads .......................................................... 59

27: $\quad$ 4-Inch Carbon Core-Hemp Skin Ultimate Stresses ....................................................... 59

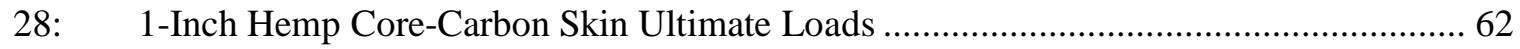

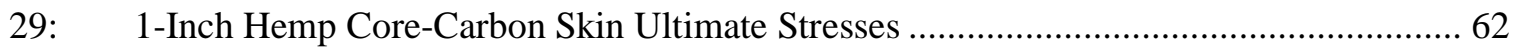

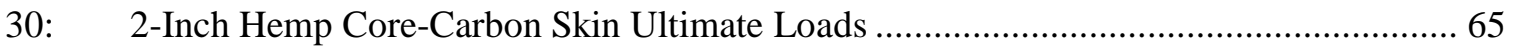




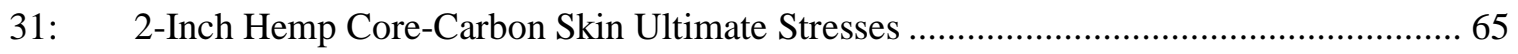

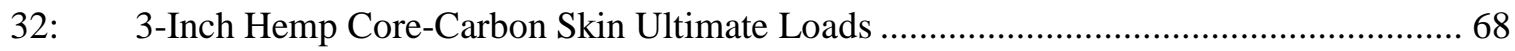

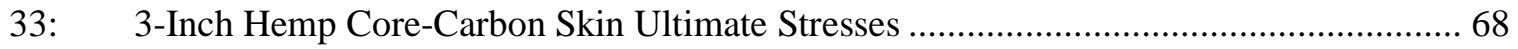

34: 3-Inch Hemp Core-Carbon Skin Sample Properties ................................................... 71

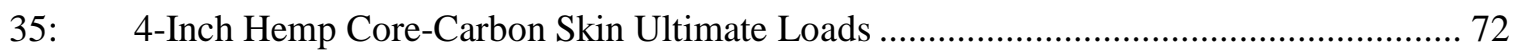

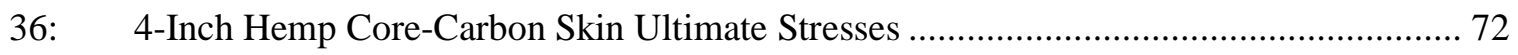

37: Average Ultimate Load Of Different Composite Configurations and Lengths................ 76

38: Average Ultimate Load Percent Differences Compared to All-Carbon Samples ............ 77

39: Average Ultimate Load Percent Differences Compared to All-Hemp Samples .............. 77

40: Average Ultimate Stress of Different Composite Configurations and Lengths ............... 79

41: $\quad$ Average Stiffness of Different Composite Configurations and Lengths.......................... 81

42: $\quad$ Average Stiffness Percent Differences Compared to All-Carbon Samples ..................... 82

43: Average Stiffness Percent Differences Compared to All-Hemp Sample ........................ 82

44: Average Ultimate Load-Over-Weight of Composite Configurations and Lengths ........... 84

45: 1- and 2-Inch Ultimate Load of Different Composite Configurations ............................ 93

46: 1- and 2-Inch Stiffness of Different Composite Configurations ................................... 93

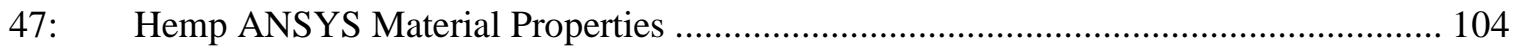

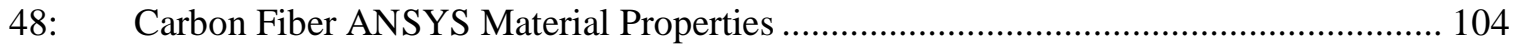




\section{LIST OF FIGURES}

Figure Page

1: $\quad$ Fiber Direction Variations in Composites ................................................................. 1

2: $\quad$ Injection Molded Bast Fiber Composite Part .............................................................. 3

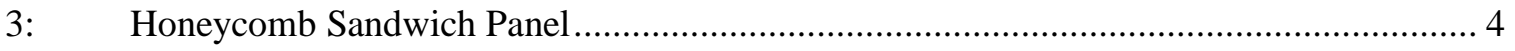

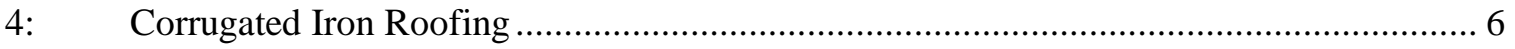

5: $\quad$ Single Layer Corrugated Sandwich Panel Construction with 5 Cells ................................ 8

6: $\quad$ Fiberglass and Carbon Fiber Corrugated Sandwich Panel Boxes ..................................... 9

7: $\quad$ Trapezoidal Corrugated Sandwich Panel, Skin Debonding Under Axial Compression ..... 9

8: $\quad$ Original Axis and Sections Used in Analysis by Section .............................................. 11

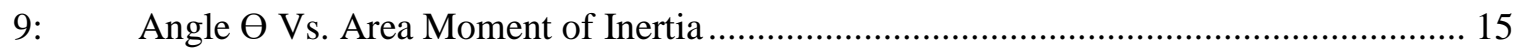

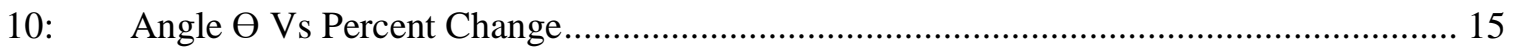

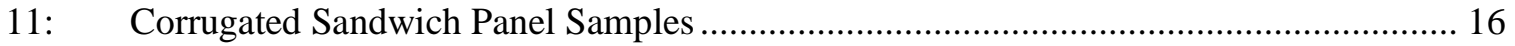

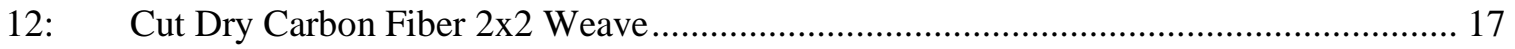

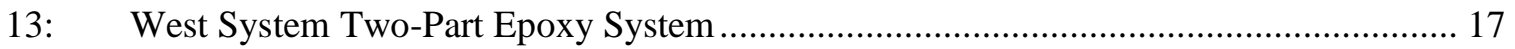

14: Corrugation Room-Temperature Vacuum Curing Setup ................................................ 18

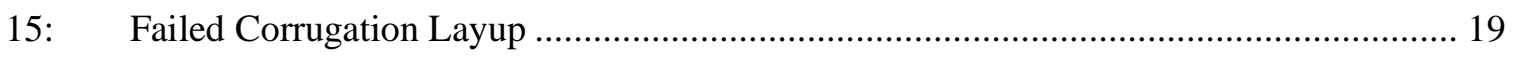

16: $\quad$ Cured Corrugated Carbon Fiber Sections ............................................................... 19

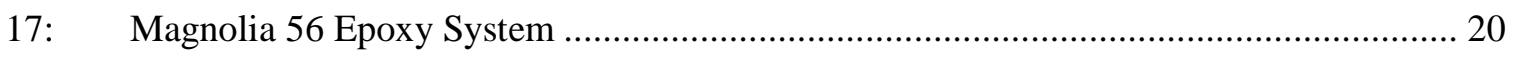

18: $\quad$ Pre-Cut and Completed 1-Inch And 2-Inch Test Samples ............................................ 22

19: $\quad$ 4-Inch Long Hemp Sample Axial Compression Test ................................................... 23

20: 1-Inch Hemp Compression Test Progression with Cardboard Buffer.............................. 24

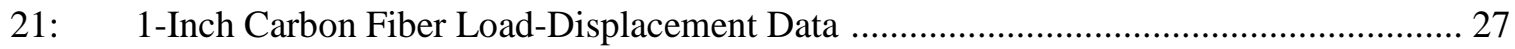

22: 1-Inch Carbon Fiber Compression Test with Edge Buckling ....................................... 27

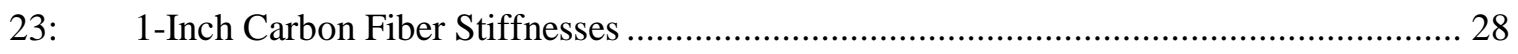

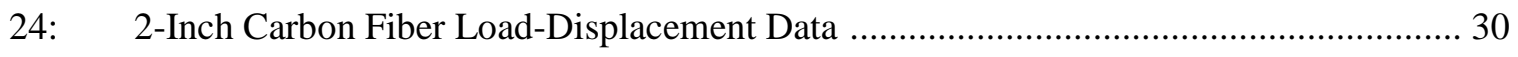

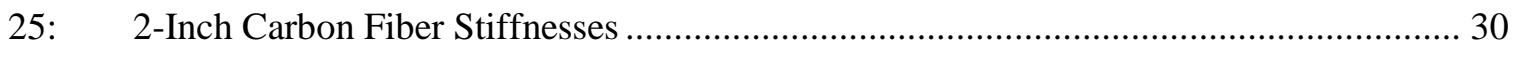

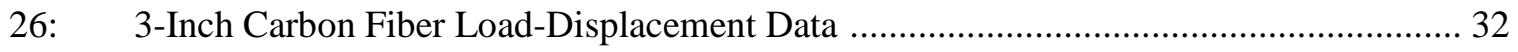

27: $\quad 3-$ Inch All-Carbon Fiber Compressed Samples ............................................................ 33

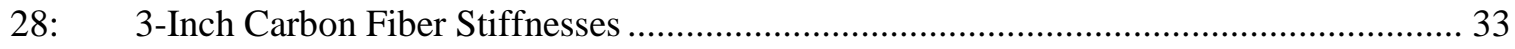

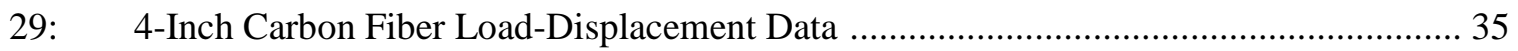

30: $\quad$ 4-Inch Carbon Fiber Samples After Axial Compression Test ....................................... 36 


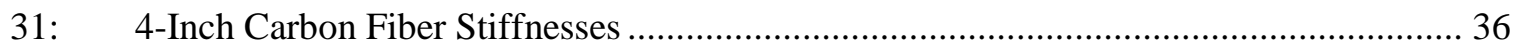

32: $\quad$ 1-Inch Hemp Fiber Compression Test with Front Face Buckling .................................. 39

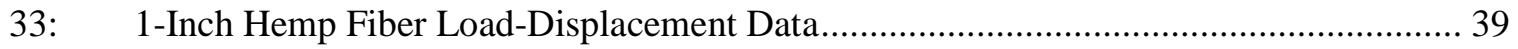

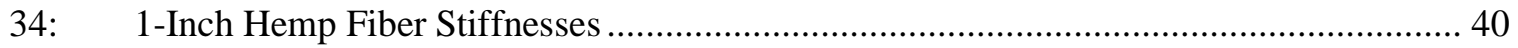

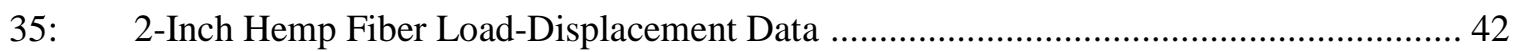

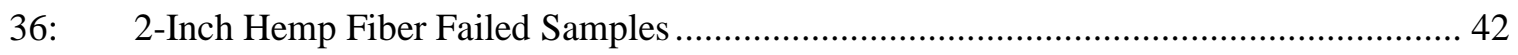

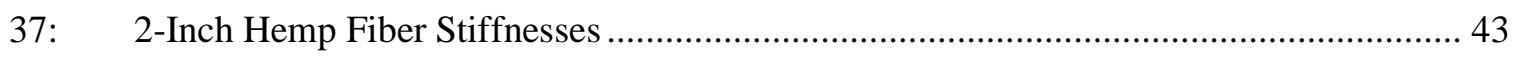

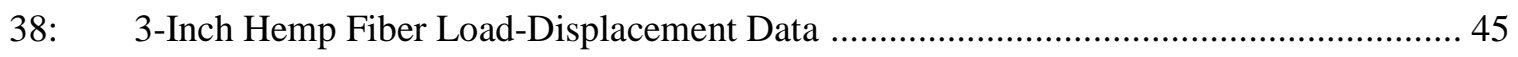

39: $\quad 3-I n c h$ Hemp Fiber Compression Test with Front Face Buckling ................................. 45

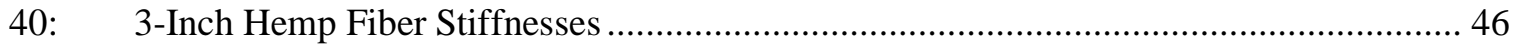

41: $\quad$ 4-Inch Hemp Fiber Load-Displacement Data …............................................................ 47

42: $\quad$ 4-Inch Hemp Fiber Compression Test with Whole Structure Buckling ......................... 48

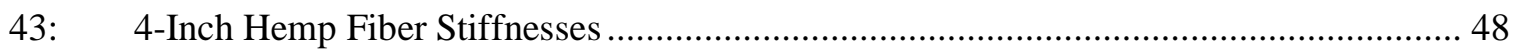

44: 1 -Inch Carbon Core-Hemp Skin Samples (1 and 2 on Bottom, 3 and 4 on Top)............ 49

45: 1-Inch Carbon Core-Hemp Skin Samples Buckling Progression Side View ................... 51

46: $\quad$ 1-Inch Carbon Core-Hemp Skin Load-Displacement Data ......................................... 51

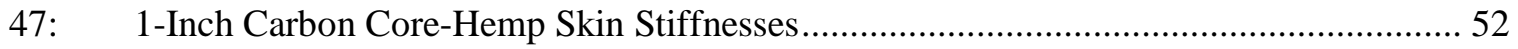

48: $\quad$ 2-Inch Carbon Core-Hemp Skin Load-Displacement Data ........................................... 54

49: 2-Inch Carbon Core-Hemp Skin Compression Test Failed Samples .............................. 55

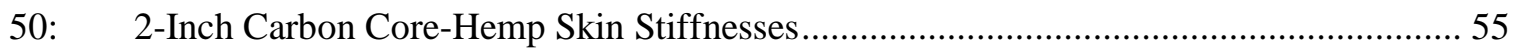

51: 3-Inch Carbon Core-Hemp Skin Load-Displacement Data ........................................... 57

52: 3-Inch Carbon Core-Hemp Skin Compression Test Failed Samples ............................... 57

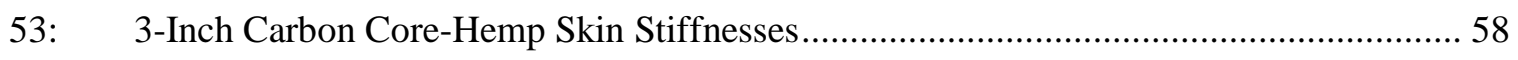

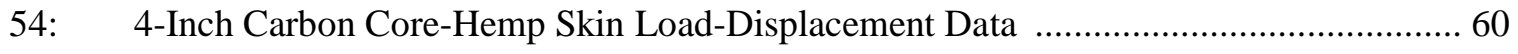

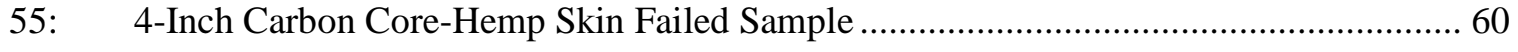

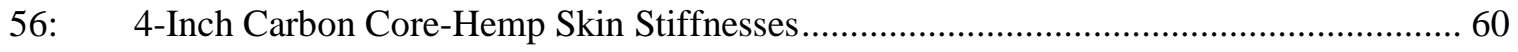

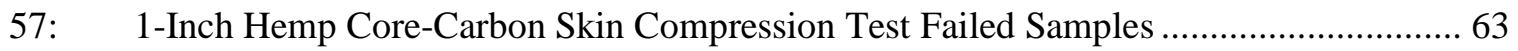

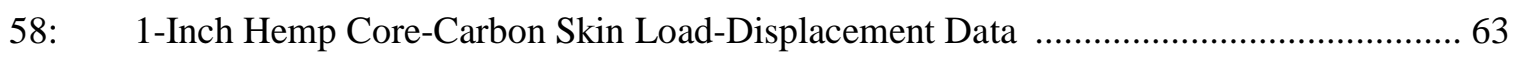

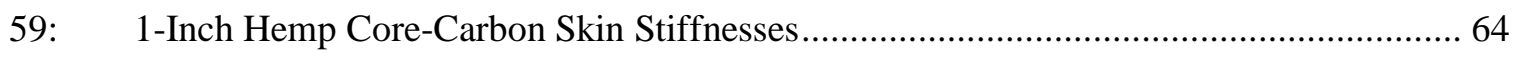

60: 2-Inch Hemp Core-Carbon Skin Compression Test Failed Samples .............................. 65

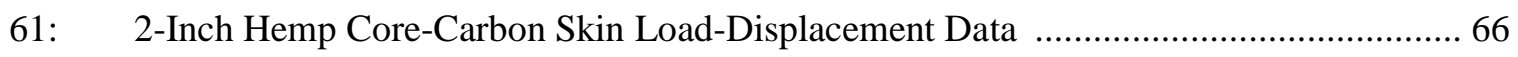

62: 2-Inch Hemp Core-Carbon Skin Compression Test Failed Samples .............................. 67

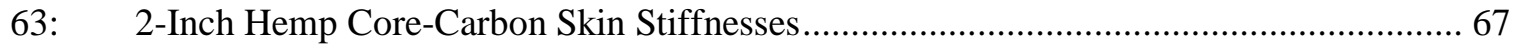




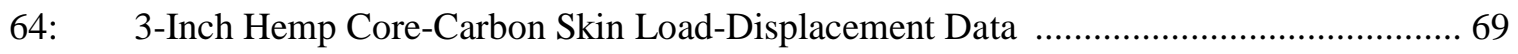

65: 3-Inch Hemp Core-Carbon Skin Compression Test Failed Samples ............................... 70

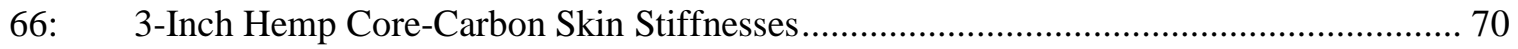

67: 4-Inch Hemp Core-Carbon Skin Compression Test Failed Samples ….......................... 72

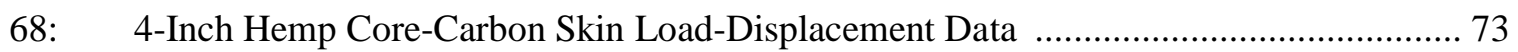

69: 4-Inch Hemp Core-Carbon Skin Compression Test Failed Samples ............................... 74

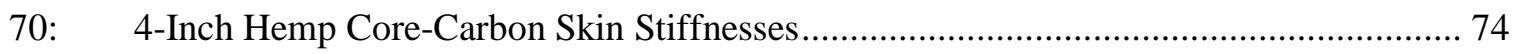

71: Average Ultimate Load Cross-Material and Cross-Length Comparison Plot ................. 78

72: Average Ultimate Stress Cross-Material and Cross-Length Comparison Plot.................. 80

73: Average Stiffness Cross-Material and Cross-Length Comparison Plot ............................ 83

74: 1-Inch All-Carbon Fiber Samples 1 And 3 With Skin-Core Debonding Failure .............. 85

75: 1-Inch Hemp Core-Carbon Skin Samples 1 And 2 Without Skin-Core Debonding ........ 86

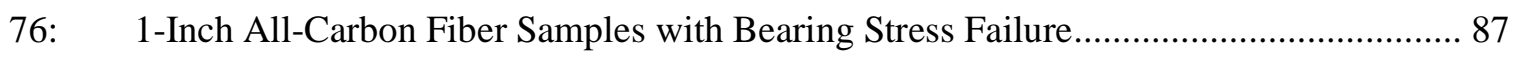

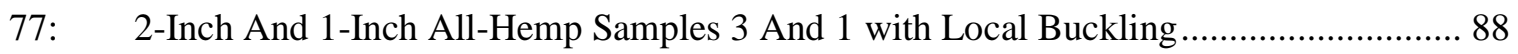

78: $\quad$ 1-Inch Hemp Core-Carbon Skin Samples 1 And 2 with Local Buckling ....................... 88

79: $\quad$ 4- And 3-Inch All-Carbon Samples with Local Buckling and Bearing Stress ................. 89

80: 3-Inch Carbon Core-Hemp Skin Samples with Local Buckling and Bearing Stress........ 90

81: 4-Inch All-Hemp And Hemp Core-Carbon Skin Samples With Global Buckling ........... 90

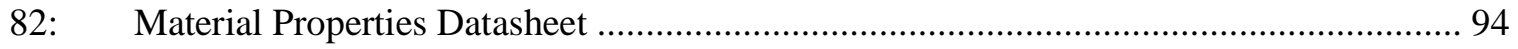

83: ANSYS Composites PrePost Static Structural and Eigenvalue Buckling Setup .............. 95

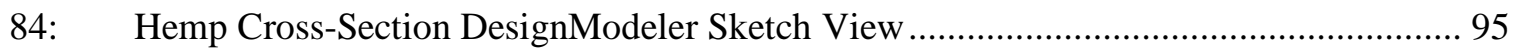

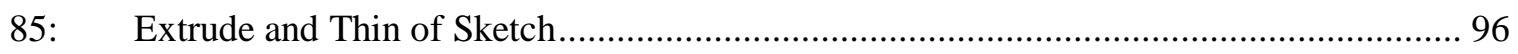

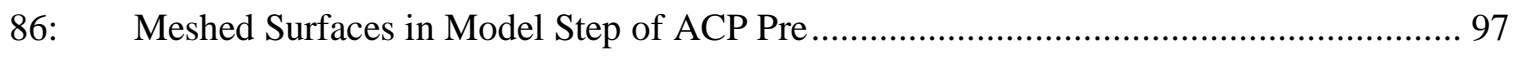

87: Assigning Material and Thickness to a Fabric in ACP Pre Setup ................................... 98

88: $\quad$ Oriented Selection Set Direction and Three Plies Laid Up From Green Surface............. 98

89: Modeling Ply Configuration in Modeling Group …................................................... 99

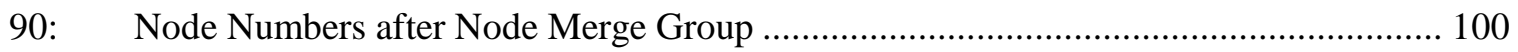

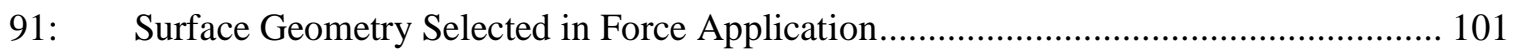

92: Surface Geometry Selected in Force Application........................................................ 102

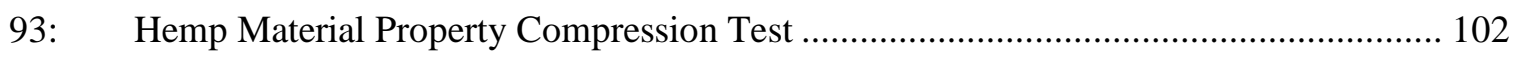

94: Hemp Compression Test for Young's Modulus (Pre-Buckling Region Outlined in Red)

95: 1-inch hemp ANSYS and Experimental Results 105 


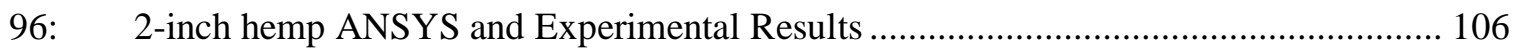

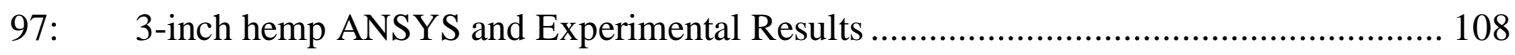

98: $\quad$ 4-inch Hemp ANSYS and Experimental Results ….................................................... 109

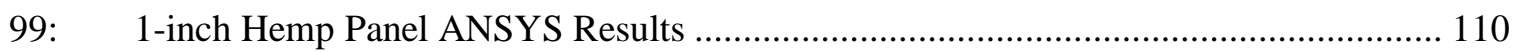

100: 1-inch Carbon Fiber ANSYS and Experimental Results ........................................... 111

101: 2-inch Carbon Fiber ANSYS and Experimental Results ............................................ 113

102: 3-inch Carbon Fiber ANSYS and Experimental Results .......................................... 114

103: 4-inch Carbon Fiber ANSYS and Experimental Results ............................................ 114

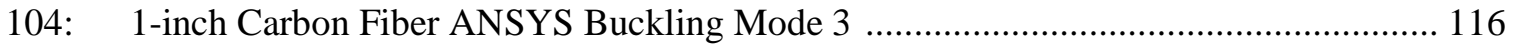

105: 1-inch Hemp Experimental Local Buckling Results................................................... 116

106: 1 -inch Carbon Fiber ANSYS Buckling Mode 1 ....................................................... 116

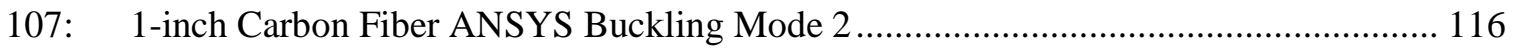

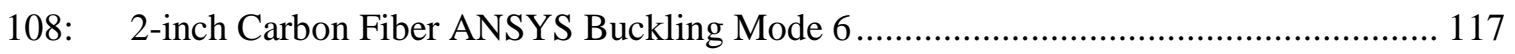

109: 2-inch Hemp Core-Carbon Skin and All-Hemp Samples with Local Buckling............. 117

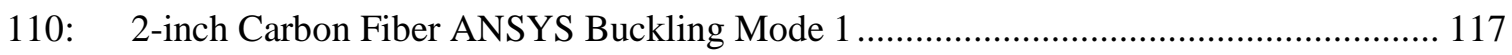




\section{INTRODUCTION}

\subsection{An Overview of Traditional Composites}

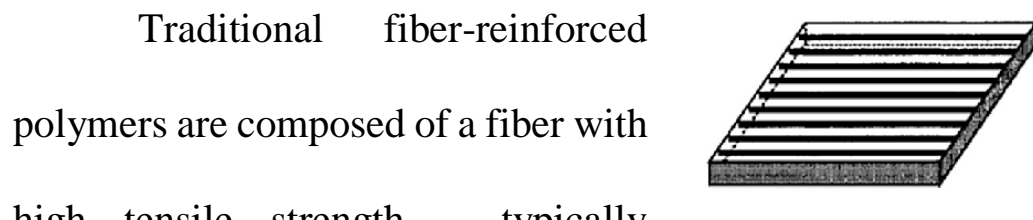

high tensile strength - typically

carbon, glass, or even steel rebar - and

a matrix which holds the fiber in

alignment - typically a polymer, like

epoxy, or concrete. This results in a

mixture of discrete materials with the (a) Unidirectional

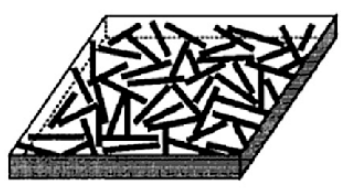

(c) Discontinuous fiber

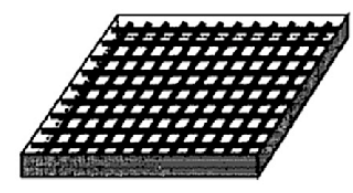

(b) Bi-directional

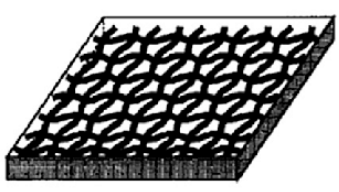

(d) Woven

Figure 1: Fiber Direction Variations in Composites

desirable properties of both. These properties can be altered not only by using different combinations of fibers and matrix, but also by changing the orientation of the fibers or the percentage of the fibers in comparison to the matrix.

The reorientation of the fibers can take the form of turning the fibers in relation to the force direction, using randomly oriented fibers, or using one of the various types of woven patterns. Some basic configurations can be seen diagrammed in Fig. 1 (Tawfik).

Fiber-reinforced polymer composites like carbon fiber and fiberglass are currently used in many applications that require low weight and high tensile strength. These include - but are not limited to - aircraft, automobiles, marine vehicles, and construction. 


\subsection{An Overview of Bio-Composites}

Like traditional fiber-reinforced polymer composites, bio-composites are composed of a load bearing fiber and a cohesive matrix. The difference, however, is the incorporation of a natural fiber as a replacement to man-made fibers like carbon, fiberglass, and aramids. Most of these-made fibers have a large ecological footprint and are expensive to manufacture, especially carbon fiber, which costs 14 times as much energy as steel to make, according to Sujit Das in "Life Cycle Assessment of Carbon Fiber-Reinforced Polymer Composites," published in The International Journal of Life Cycle Assessment. Bio-composites, on the other hand, are often byproducts of plants which results in a much smaller ecological footprint and much lower costs. They are currently used in the construction industry as "scaffolding, formwork, flooring, walls and for many other applications within buildings, as well as temporary construction" (Cristian, 1), and in the automotive industry as "reinforcement of the door panels, passenger rear decks, pillars, and boot linings" (Zhakal). Natural fiber reinforced composites, however, still have not been widely used in aerospace applications. This is partly due to the slightly diminished tensile strength, increased variability, and high moisture absorption compared to precision manufactured fibers and partly due to a resistance to the idea of and stigma attached to more widely used natural fibers like hemp.

\subsection{Hemp Fibers}

Hemp fibers, also known as bast, are the fibers on the outside of the hemp plant's stalk. These fibers provide the stalk the strength it needs to be held upright. The fibers, typically anywhere from 3 to 15 feet in length, are harvested using a series of rollers. These 
separate the bast from the woody core. Then, the fibers are cleaned of excess particles, measured and categorized for fineness, and sometimes cut to meet specific length requirements, like the short cut, randomly oriented fibers seen in Fig. 2, from the Canadian Hemp Trade Alliance.

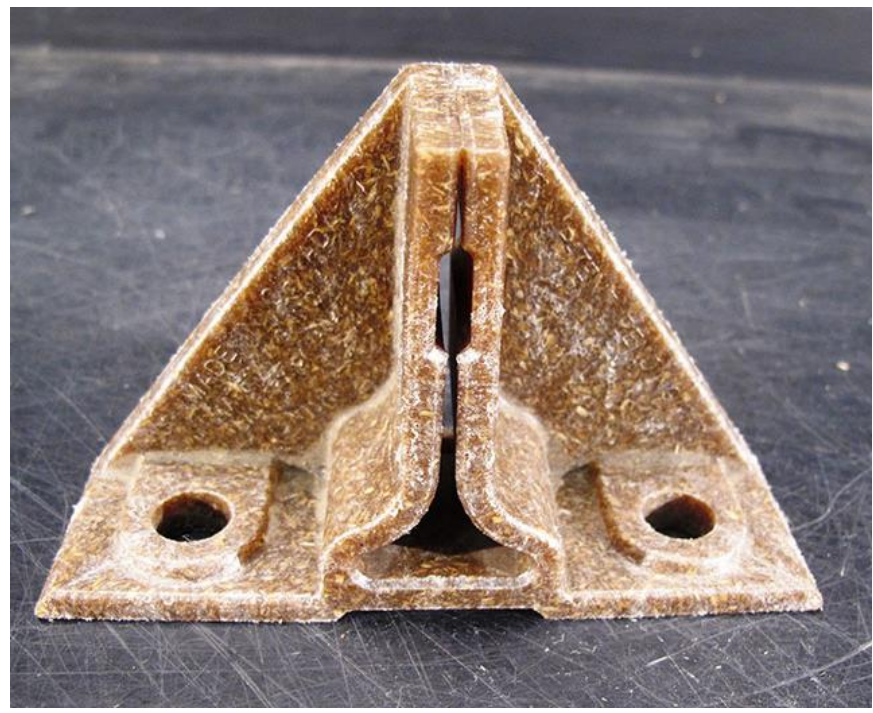

Figure 2: Injection Molded Bast Fiber Composite Part

Postprocessing, in the form of chemical treatment or matting, of these fibers is required in order to form them into tows and eventually weaves. This chemical treatment removes the natural binders between individual fibers and allows them to be separated into specific amounts to form uniform tows.

As of February 2019, 9 states do not allow the cultivation of industrial hemp, which is much different than marijuana, according to the National Conference of State Legislatures. While both are types of the cannabis plant, there are stark differences between the two, mainly the tetrahydrocannabinol (THC) - the ingredient that produces a high when smoked - and fiber contents. While marijuana can have a THC concentration of around 20 percent, most states define industrial hemp of having a concentration of less than 0.3 percent. Industrial hemp also has a very high fiber content in comparison. Despite these distinct differences and the degree to which they separate each plant's uses, they are often still confused. Industrial hemp is stigmatized, causing it to be overlooked as a useful, environmentally friendly, and cheap material. 
The use of industrial hemp fibers in reinforced polymer composites has been gaining traction in North America's scientific community since Canada legalized hemp production and sale in 1998 and as industrial hemp laws gradually relax in the United States. Globally, industrial hemp has been researched for longer as it has been legalized in many countries and it has been discovered to have many desirable material properties, in addition to the low cost and net environmental impact it boasts. It is currently being incorporated as a replacement to glass fibers as the tensile strength and stiffness it has is comparable.

\subsection{Sandwich Panel Construction}

Beyond typical
composite plates, the
construction of sandwich
panels can improve
characteristics for specific
types of loading and can help
enhance the performance of

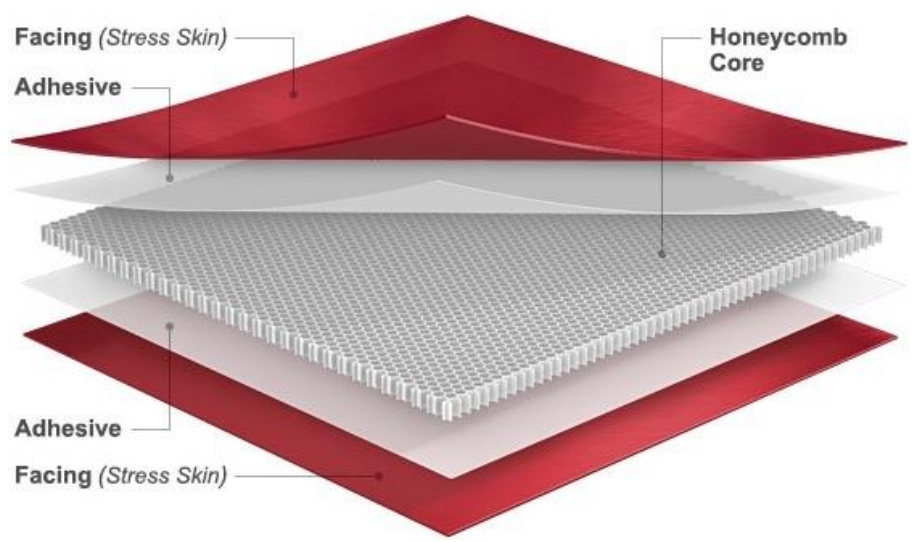

Figure 3: Honeycomb Sandwich Panel

the material for a desired application. Sandwich panel construction has been used for more than 50 years to construct lighter structures capable of bearing heavier loads. There are typically two stiff, strong skins with a lightweight core separating the two skins that can be the same or different than the two skins. Open and closed cell structured foam, aluminum, polystyrene, balsa wood, syntactic foam, honeycomb and Nomex® are common core materials. Common skin materials include glass or carbon fiber reinforced laminates. 
Separating the skins which carry the main loads of the structure with a low-density core increases the moment of inertia of the structure with slight increase in weight. This tradeoff between a higher moment of inertia and a small addition to weight produces a very efficient composite structure. A typical honeycomb sandwich panel diagram from Plascore can be seen in Fig. 3.

Sandwich panels offer higher strength-to-weight ratios than solid composite laminates alone. A common example of a composite sandwich panels are snow skis. They consist of a carbon fiber or fiberglass skin bonded to and separated by balsa wood or a rigid polyurethane foam. Sandwich panels also have applications in thermal and acoustic management of structures as well as home and building construction, boat construction, spacecraft and aircraft structures, and even automobiles. The core material acts as an insulator between the two skins, resisting changes in heat and absorbing sound energy.

However, sandwich panels made using traditional cores like those above are subject to delamination due to bonding failure between two dissimilar materials lacking in bonding surface area and thus need to have more surface area, resulting a denser core. This problem is mitigated when using corrugated structures that run parallel to the skin. Corrugated structures are noted for their exhibition of extreme anisotropic stiffness properties deeming them particularly useful in structural applications. As stated earlier, the addition of a core between two composite skins increases the structures moment of inertia and strength-toweight ratio but having a stronger bond between skin and core allows a lighter core to be used, further decreasing the overall weight. The proven advantage of corrugated sandwich panels lies in the decreased weight and decreased weakness to delamination. 
Again, biomaterials such as hemp, corn husks, and bamboo have been given much more weight in the scientific community lately due to their comparable strength to weight ratio to manufactured, non-sustainable materials, and their extremely low cost. In considering usage of a biomaterial in a sandwich panel, there are three distinct options usage in the skin, the core, or the whole sandwich panel construction. All will be discussed further in this research.

\subsection{An Overview of Corrugation}

Corrugation has long been used to increase the structural properties of thin sheets. From "History of Corrugated Iron" in World Archaeology, the first form of corrugation was first patented in 1829, when "indented or corrugated metallic sheets" of iron were used as a lightweight, yet strong, roofing solution for warehouses at the London Docks (Miles). These corrugated iron sheets were manufactured by pressing iron through fluted rollers. This can be seen in Fig. 4, from Robot Building Supplies.

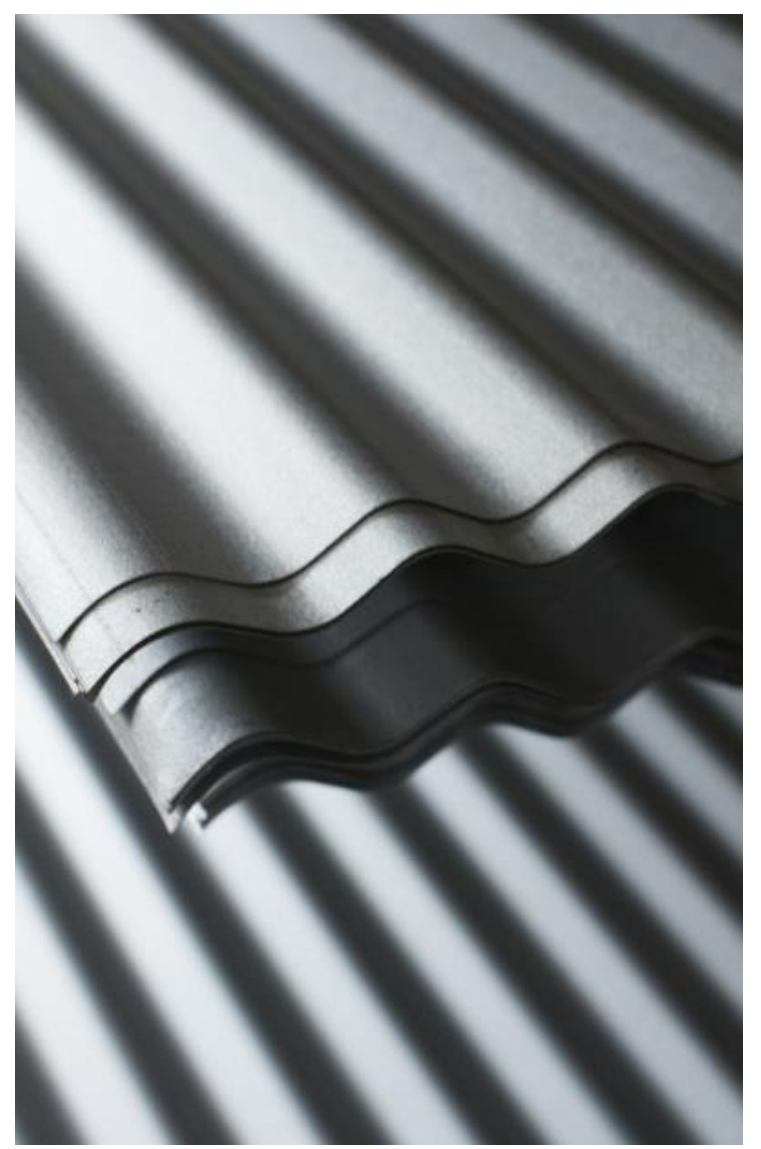

Figure 4: Corrugated Iron Roofing 
Compared to a thin flat plate, corrugation has a much higher area moment of inertia along the vertical axis. A thin flat plate almost has no dispersal of the cross-sectional area in the vertical direction, but corrugating the plate adds groves in the material, dispersing some of the cross-sectional area up and down the vertical axis. This increases the area moment of inertia, or the capacity of the cross section to resist bending. The equation for the area moment of inertia about the horizontal axis can be seen below.

$$
\text { (1) } I_{x}=\int_{A} y^{2} d A
$$

As more cross-sectional area moves away from the centroidal axis, the area moment of inertia - and the bending and buckling resistance - increases.

The strength of corrugation also can be explained through the theorem of Gaussian curvature. By curving the plate in one direction or curving it many times in one direction for the case of corrugation, it becomes stiff in the other direction. In order to bend the corrugated panel about the axis perpendicular to the corrugation, a section of the corrugation would have to lie flat about the bending axis or the plate would have to deform or fracture. 


\subsection{Corrugated Sandwich Panels}

Corrugated sandwich panels take advantage of the stiffness and strength-to-weight ratio of both sandwich panels and corrugation by replacing the traditional edge-bonded honeycomb core with a face-bonded corrugated core. This method of construction is most commonly seen in cardboard but has recently become more widely investigated as having usage in the automotive and aerospace industries. While much like other sandwich panels, corrugated sandwich panels excel in having a much higher bonding surface area - and thus core-skin bond strength - than honeycomb panels and can utilize composite materials like carbon fiber and fiberglass as the core, as well as the skin. Typical sandwich panel construction with trapezoidal corrugation can be seen below in Fig. 5, from "Crashworthiness optimization of corrugated sandwich panels" by Hou. An example of trapezoidal sandwich panels can be seen in Fig. 6. Using identical materials in both the skin and the core can be advantageous, as these materials bond well to themselves. This bond strength is important in bending resistance but, as discovered in previous research, can be a key factor in ultimate load reduction under edgewise compression.

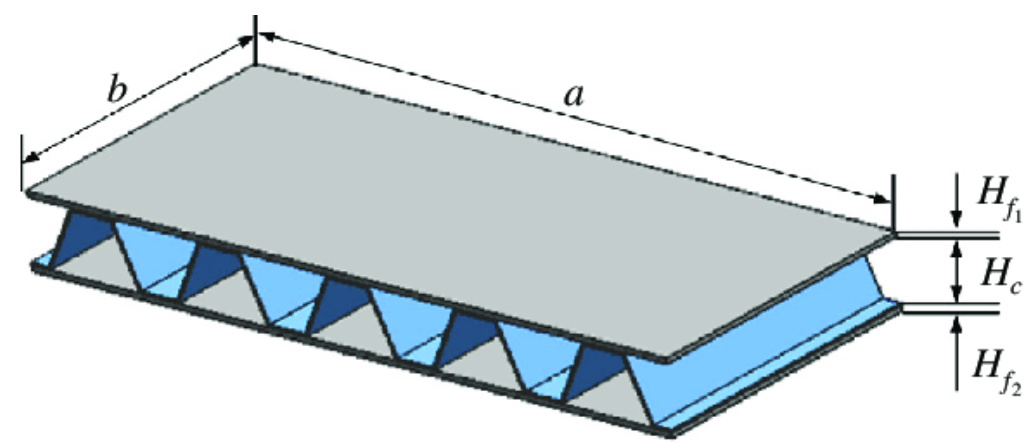

Figure 5: Single Layer Corrugated Sandwich Panel Construction with 5 Cells 


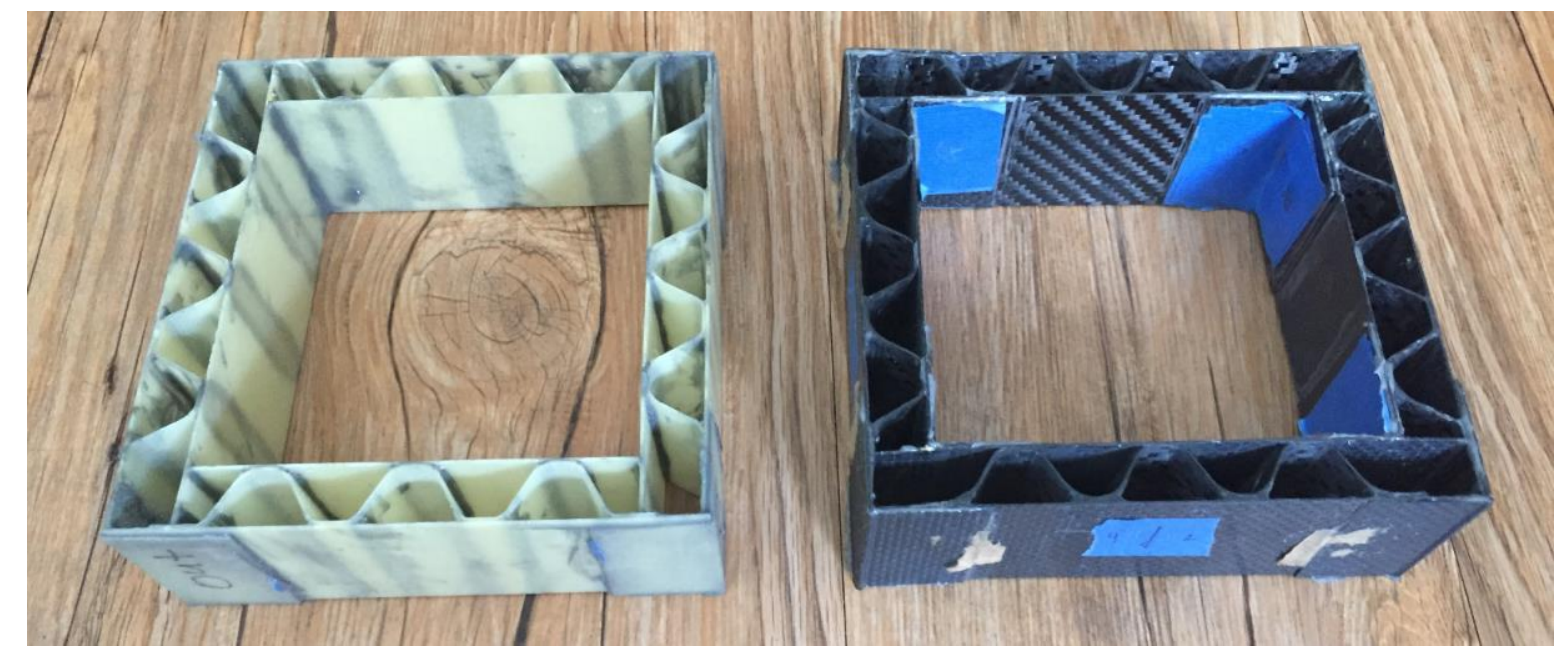

Figure 6: Fiberglass and Carbon Fiber Corrugated Sandwich Panel Boxes

\subsection{Research Objectives}

In previous research, carbon fiber and fiberglass corrugated structures subjected to edgewise compression testing experienced several different early failure modes as the skins debond from the core. This is thought to be due to the massive amount of tension put on the bond as the identical stiffness of the two faces causes buckling in opposite directions. This debonding, seen in Fig. 7 on a 2inch carbon fiber weave sample, results in complete separation of the

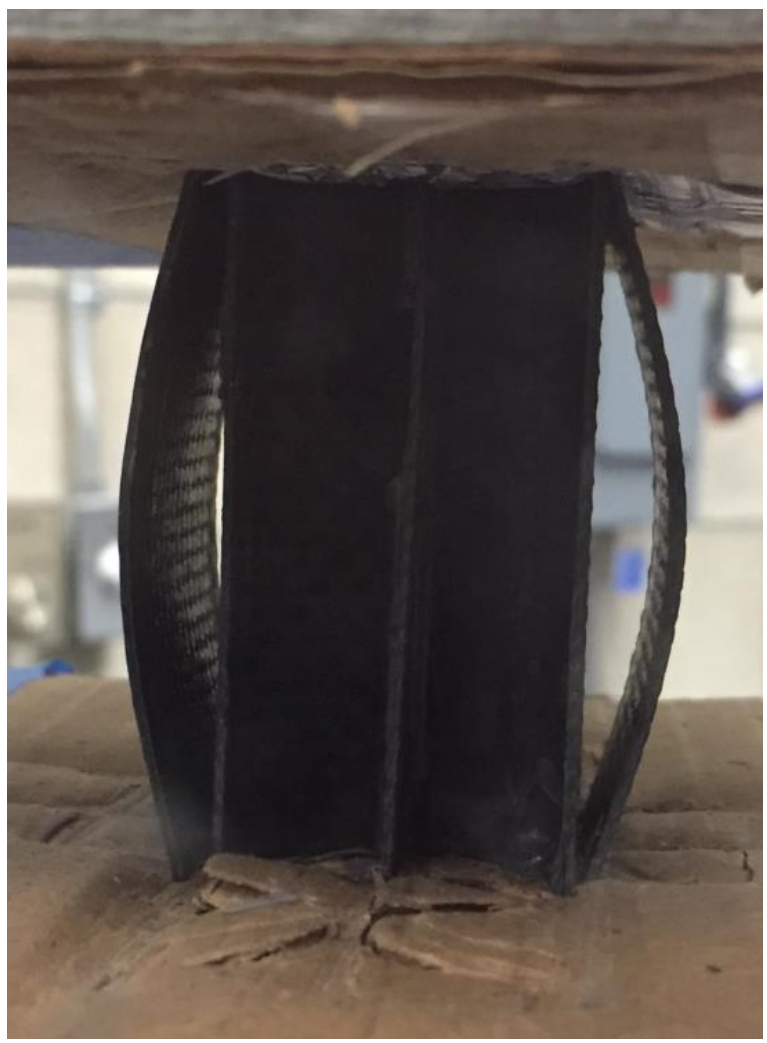

Figure 7: Trapezoidal Corrugated Sandwich Panel, Skin Debonding Under Axial Compression 
sample into parts and diminished strength.

In order to prevent early failure, integrated hemp composites would allow for different stiffnesses and less tension on the bond. This would allow the structure to withstand greater axial compression while making the structure cheaper and more environmentally friendly.

Thus, the primary goals of this research are determining hemp has comparable enough mechanical behavior to traditional composites to serve as a viable replacement and if integrating hemp sections into the sandwich panel solve this debonding problem. The secondary goals are to see at what length buckling behavior became an issue and to see if this corrugated structure and all its failure modes could be simulated in finite element analysis. To accomplish this, edgewise compression testing will be performed on carbon fiber, hemp, and mixed corrugated sandwich panels of varying lengths from 1 to 4 inches.

It is hypothesized that corrugation will help sustain high loads for a significant amount of time post-failure, even further with the usage of hemp fiber composites. Hemp fibers will also increase the resilience of the structure to debonding failure modes due to the greater ductility of the material. 


\section{CORRUGATION GEOMETRY THEORETICAL ANALYSIS}

\subsection{Optimizing Cross-Sectional Shape with MATLAB Analysis}

Choosing geometry was a large decision in this experiment since each geometry has different mechanical and bonding properties that must all be considered. For this reason, three main categories of geometries were selected, and shapes were chosen based on a constant cross-sectional area. The three major considerations for this experiment were stability in buckling, which can be measured to some degree by the area moment of inertia, the resistance to delamination between corrugated sections and plates, which can be measured by an approximation of the bonding surface area, and the susceptibility of the geometry to stress concentrations.

The first form of analysis was of the area moment of inertia of each shape. For this, the depth, thickness, and area were held constant, in this case at 8.66025 units, 0.1 units, and 4 units. Theoretically, this allows change in geometry without any change in the mass of the final test sample. First, the original axis locations were chosen, seen below in Fig. 8 . Then, for each shape, measurements of sections were used, with the constants defined above, and the area moments of inertia were calculated using the parallel axis theorem.

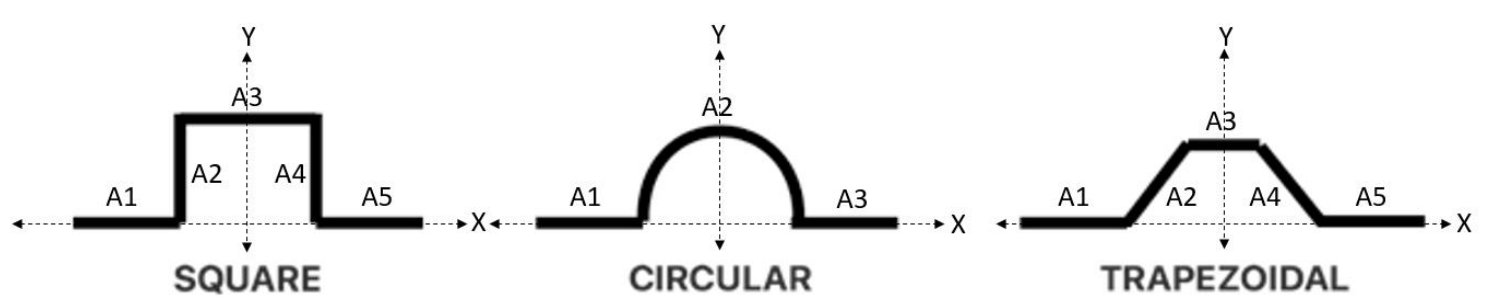

Figure 8: Original Axis and Sections Used in Analysis by Section 
These measurements can be seen below in Tables 1,2, and 3, where all the numbers used in area moment of inertia calculations for each of the considered geometry possibilities are displayed. From this data, the geometry with the highest total area moment of inertia was valued. The sections of each shape are labelled on Fig. 8 and correspond to the values in Tables 1,2, and 3. For this process, the moment of inertia about the shown $\mathrm{x}$ axis was first calculated and then the parallel axis theorem was used to determine the moment of inertia about the centroid.

In these tables, $I_{x \_} b a r$ is the area moment of inertia about the centroidal $\mathrm{x}$-axis of each section of a cross-sectional shape, as labeled by the ID and seen in the Fig. 8 above. Then, this value for each section is added to the quantity of the area of the section multiplied by the centroid location of the section in regards to the original $\mathrm{x}$-axis along the bottom of the cross-section, also seen in Fig. 8. These values can be added up to calculate the total moment of inertia about the original $\mathrm{x}$-axis and then converted to the total moment of inertia about the centroid of the entire cross section.

While the bonding surfaces of the trapezoidal and the rectangular can be closely matched. However, the circular cross section could not. While the arch has proven structural stability along the vertical axis, it would give in to delamination under compression very easily due to the nearly non-existent flat portion on the top of the semicircle. Thus, the circular cross section was eliminated. 
Tables 1, 2, \& 3: Trapezoidal, Rectangular, and Circular Cross-Sectional Area Moments of Inertia

\begin{tabular}{|c|c|c|c|c|c|c|}
\hline \multicolumn{7}{|c|}{ Trapezoidal (60 degrees) } \\
\hline ID & Area & $y_{i}$ & $y_{i}^{*}$ area & $I_{x}$ bar & $A^{*} y_{i}{ }^{2}$ & $I_{x}$ bar $+A^{*} y_{i}^{2}$ \\
\hline A1 & 0.5 & 0 & 0 & 0.000833 & 0 & 0.000833333 \\
\hline A2 & 1 & 4.330127 & 4.330127 & 5.412182 & 18.75 & 24.16218247 \\
\hline A3 & 1 & 8.660254 & 8.660254 & 0.000833 & 75 & 75.00083333 \\
\hline A4 & 1 & 4.330127 & 4.330127 & 5.412182 & 18.75 & 24.16218247 \\
\hline A5 & 0.5 & 0 & 0 & 0.000833 & 0 & 0.000833333 \\
\hline Totals & 4 & 17.32051 & 17.32051 & & & \\
\hline \multicolumn{3}{|c|}{ Y-position of centroidal X-Axis } & 4.330127 & & & \\
\hline \multicolumn{3}{|c|}{$I_{x}$ (Inertia about original X-Axis) } & 123.3269 & & & \\
\hline \multicolumn{3}{|c|}{$\mathrm{I}_{\mathrm{x} \_ \text {bar (Inertia about centroid) }}$} & 48.32686 & & & \\
\hline
\end{tabular}

\begin{tabular}{|c|c|c|c|c|c|c|}
\hline \multicolumn{7}{|c|}{ Rectangular } \\
\hline ID & Area & $y_{i}$ & $\mathrm{y}_{\mathrm{i}}^{*}$ area & $I_{x}$ bar & $A^{*} y_{i}^{2}$ & $I_{x \_}$bar $+A^{*} y_{i}^{2}$ \\
\hline $\mathrm{A} 1$ & 0.56699 & 0 & 0 & 0.000833 & 0 & 0.000833333 \\
\hline $\mathrm{A} 2$ & 0.866025 & 4.330127 & 3.75 & 5.412659 & 16.23798 & 21.65063509 \\
\hline A3 & 1.13397 & 8.660254 & 9.820468 & 0.000945 & 85.04775 & 85.04869498 \\
\hline A4 & 0.866025 & 4.330127 & 3.75 & 5.412659 & 16.23798 & 21.65063509 \\
\hline A5 & 0.56699 & 0 & 0 & 0.000833 & 0 & 0.000833333 \\
\hline Totals & 4.000001 & 17.32051 & 17.32047 & & & \\
\hline \multicolumn{3}{|c|}{ Y-position of centroidal X-Axis } & 4.330116 & & & \\
\hline \multicolumn{3}{|c|}{$I_{x}$ (Inertia about original $\left.X-A x i s\right)$} & 128.3516 & & & \\
\hline \multicolumn{3}{|c|}{$\mathrm{I}_{\mathrm{x}}$ bar (Inertia about centroid) } & 53.35199 & & & \\
\hline
\end{tabular}

\begin{tabular}{|c|c|c|c|c|c|c|}
\hline \multicolumn{7}{|c|}{ Circular (Half Arc) } \\
\hline ID & Area & $y_{i}$ & $\mathrm{y}_{\mathrm{i}}^{*}$ area & $I_{x}$ bar & $A^{*} y_{i}^{2}$ & $I_{x \_}$bar $+A^{*} y_{i}^{2}$ \\
\hline $\mathrm{A} 1$ & 0.647544 & 0 & 0 & 0.000833 & 0 & 0.000833333 \\
\hline $\mathrm{A} 2$ & 2.704891 & 5.48152 & 14.82691 & 18.84988 & 81.27401 & 100.1238936 \\
\hline$A 3$ & 0.647544 & 0 & 0 & 0.000833 & 0 & 0.000833333 \\
\hline Totals & 4 & 5.48152 & 14.82691 & & & \\
\hline \multicolumn{3}{|c|}{ Y-position of centroidal X-Axis } & 3.706728 & & & \\
\hline \multicolumn{3}{|c|}{$I_{x}$ (Inertia about original $X$-Axis) } & 100.1256 & & & \\
\hline \multicolumn{3}{|c|}{$\mathrm{I}_{\mathrm{x}}$ bar (Inertia about centroid) } & 45.16622 & & & \\
\hline
\end{tabular}


The final point of analysis on these cross sections was the susceptibility to stress concentrations. This was much more qualitative than quantitative as it is commonly known that sharp bends and angles in materials create higher stress concentrations. Seeing as these cross sections would be made of layers of carbon fiber, these concentrations would be greater in the rectangular cross section than the trapezoidal cross section due to the high angle at which the fibers are being bent. Also, if pressure applied along the vertical axis is accompanied by even a slight amount of lateral force, the whole rectangular corrugated shape would be susceptible to collapse, as the vertical sections would be very unstable. These two points of analysis ultimately led to the selection of the trapezoidal cross section as the ideal geometry.

After determining the optimum corrugation shape, the trapezoid acute angles needed to be determined. This was done based on three criteria: the $2^{\text {nd }}$ area moment of inertia about the cross-section's centroid, the bonding surface area, and the severity of stress concentrations in bends. One current mold that had worked well in previous testing was an aluminum trapezoidal corrugation mold with interior angles of 63 degrees. Aluminum, along with thorough cleaning and the use of a release agent, had worked very well in previous research for wet layups, as opposed to using a treated foam or fiberboard.

For this, MATLAB analysis was done on trapezoidal angles, seen in Fig. 9 and 10 as $\Theta$, ranging from 20 to 90 degrees while holding both area, thickness, and depth constant. Fig. 9 shows the change in the area moment of inertia when varying the shown angle, $\Theta$. Fig. 10 Shows the change of the moment of inertia from degree to degree as a percentage of the max moment of inertia at $\Theta=90^{\circ}$. The goal of this was to find at which angle the increase in area moment of inertia of the trapezoidal section start to level off while still 
maintaining ample bonding surface area atop the trapezoid. This would be the point at which the trapezoidal angle has the maximum area moment of inertia while maintaining low stress concentrations. This angle was determined to be at 63 degrees. At this point, the change in area moment of inertia dips below $0.6 \%$ of the max per degree. This rate was seen to be as the point where there is minimal gain from increasing the angle. The molds for manufacture were then created with this angle.

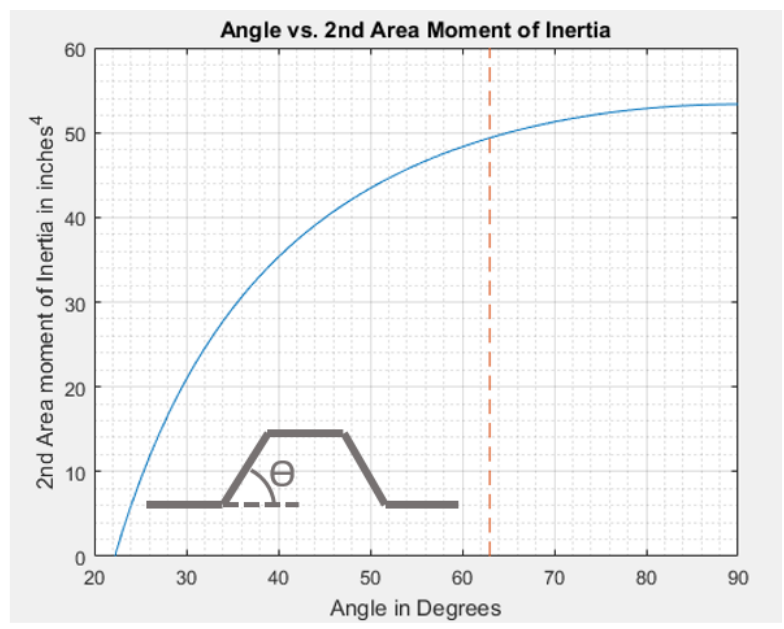

Figure 9: Angle $\Theta$ vs. Area Moment of Inertia

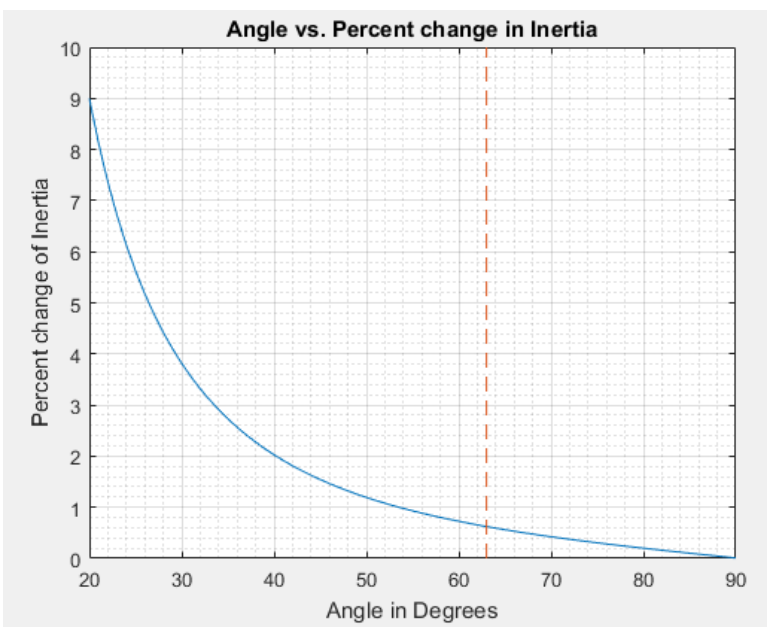

Figure 10: Angle $\Theta$ vs Percent Change 


\section{MANUFACTURING PROCESS}

Following preliminary analysis and design, the manufacturing process could start for the structures to be tested. The preliminary analysis and design phases were completed to help justify the design decisions regarding material selection, corrugation type, and manufacturing process. The corrugated test samples were varied as seen in Fig. 11. These combinations were the controls - the all carbon fiber and all hemp constructions - and the experimental variations - the hemp skin, carbon core and the carbon skin, hemp core constructions. The corrugated samples to be manufactured were to be 1 inch to 4 inches tall (along the direction of the corrugation) to investigate buckling behavior or lack thereof. Test lengths were stopped at 4 inches in due to the inability to control and mitigate eccentric loading behavior throughout the test.

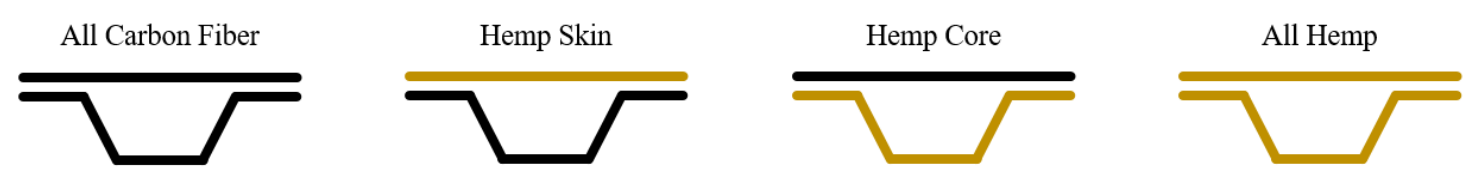

Figure 11: Corrugated Sandwich Panel Samples

\subsection{Corrugation Manufacturing Process}

Fabrication began with utilizing the dry material and room temperature vacuum pump process to form the corrugated plates. First, the molds were cleaned with acetone to help remove any oil or resin, and then prepped with a release agent to ensure the wet fabric would not stick to the mold. Next, the dry fabric, either hemp or carbon fiber, was sized and cut to fit in the mold with ample extra material to cut, seen in Fig. 12. Then, the two- 
part epoxy resin, seen in Fig. 13 was mixed and applied to the material which was then stacked, each laminate having 3 plies. This was determined to be a viable thickness while still maintaining drapability over the angles in the corrugation mold. These sheets were placed into the molds, vacuum bagged, and vacuumed for at least 24 hours. It was critical to ensure that the sheets properly conformed to the mold, so the male mold was used in tandem with a weight to assist conformity, seen in Fig. 14.

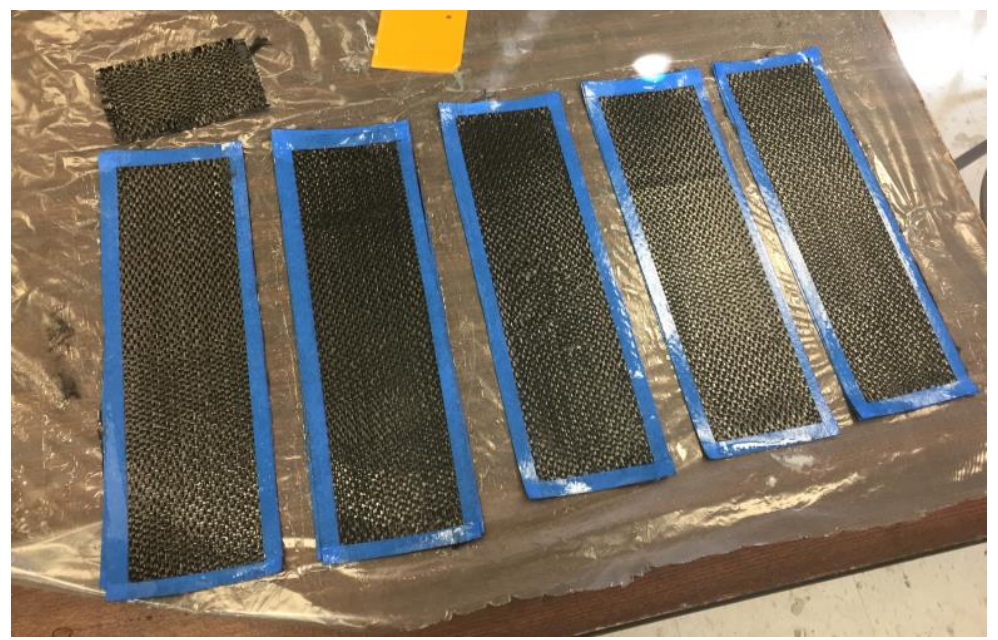

Figure 12: Cut Dry Carbon Fiber 2x2 Weave

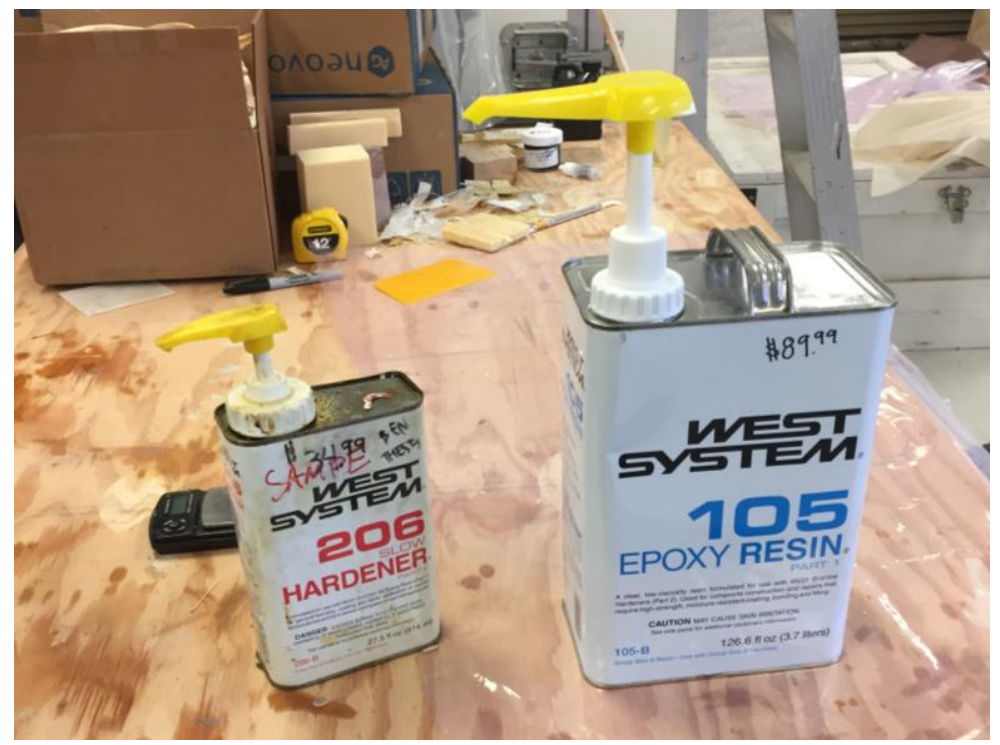

Figure 13: West System Two-Part Epoxy System 


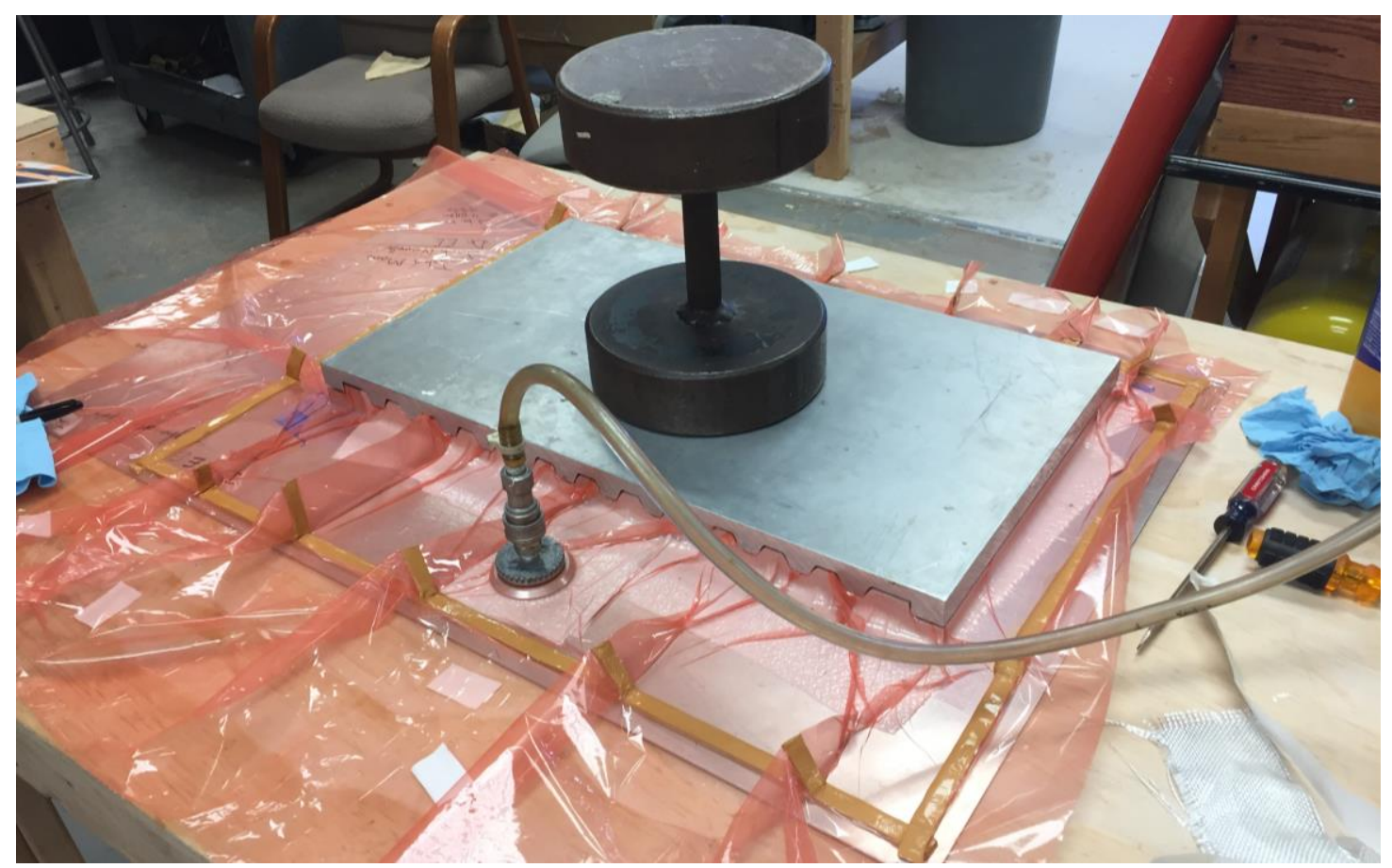

Figure 14: Corrugation Room-Temperature Vacuum Curing Setup

The first manufacture of the corrugated sections was done with a three-ply plate that covered the majority of the mold. Due to the tension in the laminate caused by the corrugation and the continuous nature of the plate, the carbon fiber had very poor conformity and various dry spots formed throughout the corrugation. This uneven distribution of resin, combined with the lack of a distinct trapezoidal shape, caused this corrugation to become unusable.

The round shape of the unconfirmed lower sections of the corrugation would not allow the right amount of bonding surface area as well. This can all be seen in Fig. 15 . After it was confirmed that the conformity was negatively being affected by the single laminate approach, the layup was changed to use strips instead of a sheet, seen in Fig. 16. This allowed the material to conform much more easily and the resin distribution and overall shape was much better. 


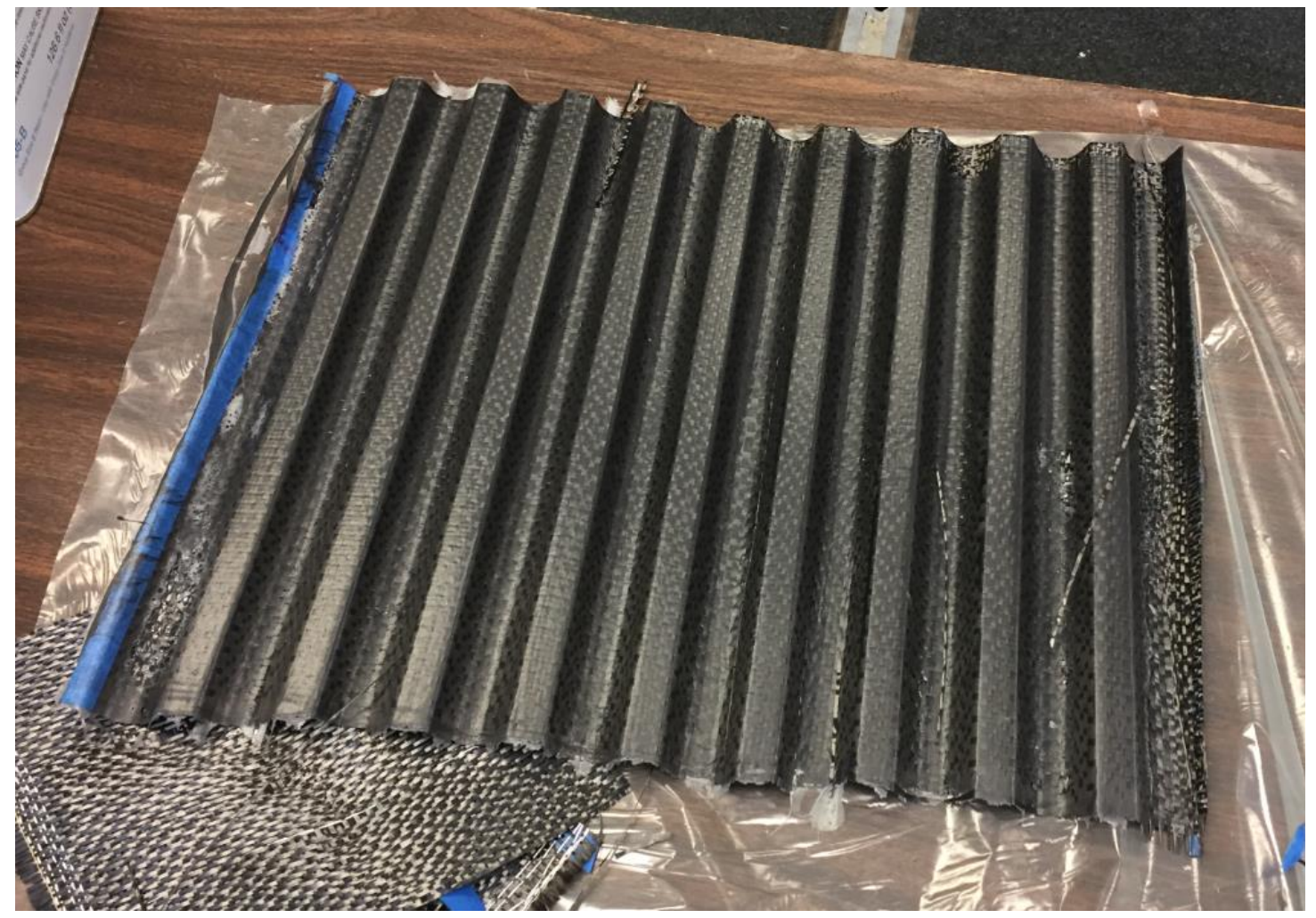

Figure 15: Failed Corrugation Layup

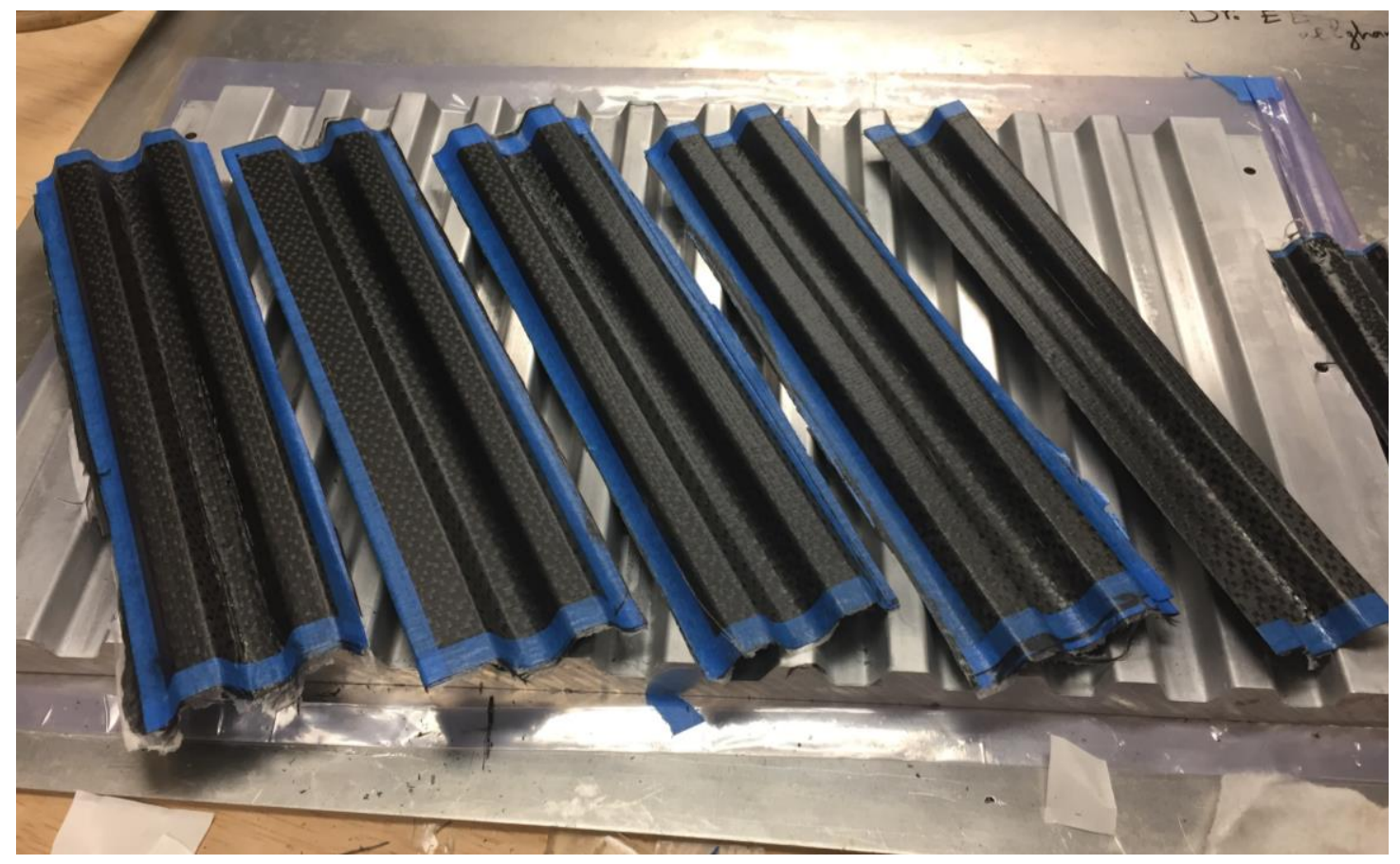

Figure 16: Cured Corrugated Carbon Fiber Sections 


\subsection{Flat Plate Manufacturing Process}

After creating the corrugation, the flat panels were made by a simpler method. Stacks of the same number of layers for each carbon and hemp were cut and impregnated with the same two-part resin. These were then cured using the same vacuum setup on a prepared flat tool plate. This included using the same layup schedule as seen in Fig. 14 the same amount of bleeder and breather - to ensure similar fiber volume fractions. This also included ensuring no bag leaks and a vacuum held for at least 24 hours.

\subsection{Assembly}

To attain the desired geometry, these flat panels were then bonded to the corrugated sections. This was done using epoxy that provides high shear strength, seen in Fig. 17. This high shear strength epoxy was used due to previous studies showing multiple failure modes

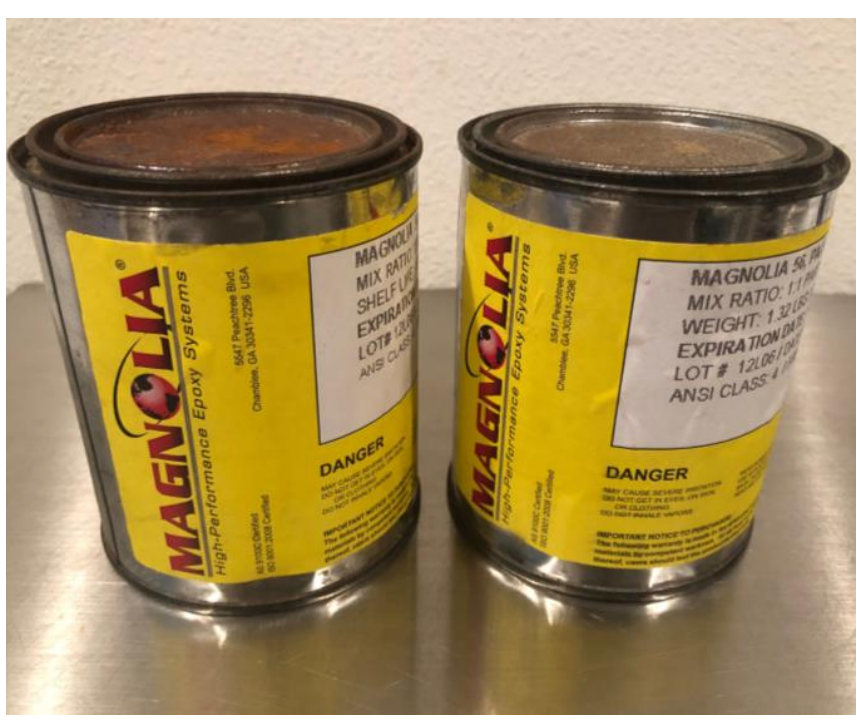

Figure 17: Magnolia 56 Epoxy System

due to debonding by shear of trapezoidal corrugated axial compression test samples. To ensure a secure bond, the epoxy was applied to roughened and cleaned surfaces on both the corrugated sections and the plates, and then applying weight on top of the structure. The surfaces were roughened with sandpaper to increase bonding surface area further. Some important steps in this process were to clean the excess epoxy out from the dips in 
the corrugation and to check to make sure that the stack is straight, and the corrugated peaks line up before carefully putting weight on top of the stack. These weights were left overnight as the epoxy bonded, and the stacks were checked sporadically for the first part of their curing cycle to ensure that the plates had not shifted as the epoxy became distributed and that the epoxy had distributed evenly.

After initial the initial bond had been completed and the epoxy completely dried, the bond was checked for any bubbles or gaps that could be filled by adding more epoxy back into the gaps. This was done to ensure that the debonding failure modes seen in previous studies would be minimized and the failure of the sample would come from solely the material and geometry. The ideal for this would be a single layup with no bonding epoxy needed. This, however, would be very difficult with the desired geometry so extra epoxy, in addition to the very strong epoxy already used, was the next best solution.

The entire fabrication process of the fully corrugated stacks was a multi-day process, being as portions had to be bonded and cured before they could be cut, to ensure proper alignment of the entire structure. Once the complete assembly was properly bonded and cured, the test samples could be cut. In order to ensure experimental validity, ideally, three samples of each case would be cut, for a total of 48 test samples. It was also important to make sure all cuts were flat, and the same amount of flange was left on the outside of each sample so that stress concentrations would not occur due to uneven loading and that the bond strengths could be compared.

Due manufacturing time and material limitations, the maximum number of samples were created with an emphasis on the shorter samples where buckling behavior would be minimized. Since the corrugation was laid up in long single sections, these were cut so that 
all the lengths would have samples but not necessarily the same number of each could be manufactured. The finished samples can be seen below in Fig. 18 .

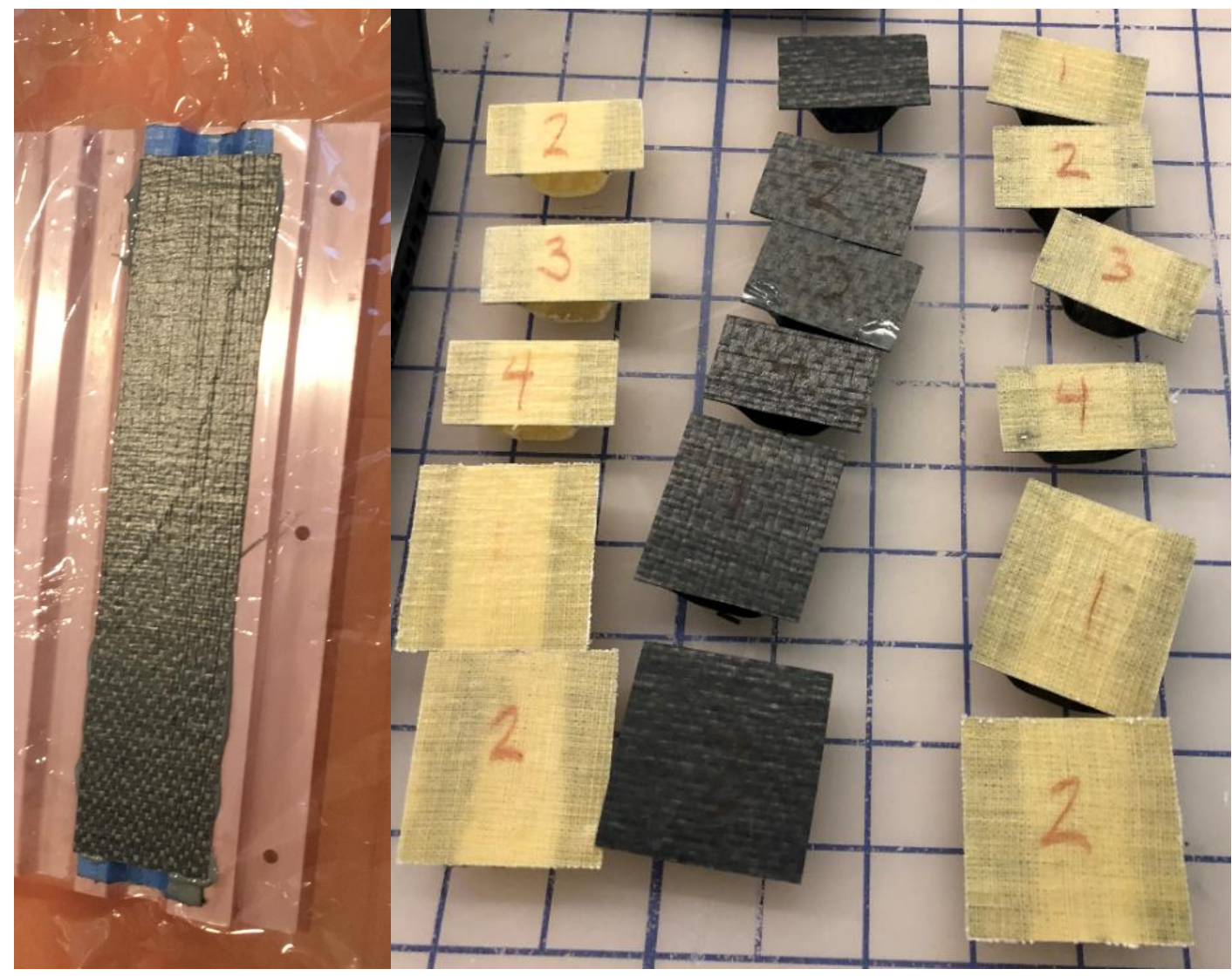

Figure 18: Pre-Cut and Completed 1-Inch and 2-Inch Test Samples 


\section{EXPERIMENTAL SETUP}

\subsection{Axial Edgewise Compression Test}

The experimental process for this experiment revolved around three primary components: preliminary analysis and design, layup and assembly, and component testing. After the layup and assembly phase, the samples and the structure needed to be prepared prior to testing. The testing phase, as the name implies, revolved around performing the axial compression test on each of the samples. The test was performed using an Instron 8801 axial test

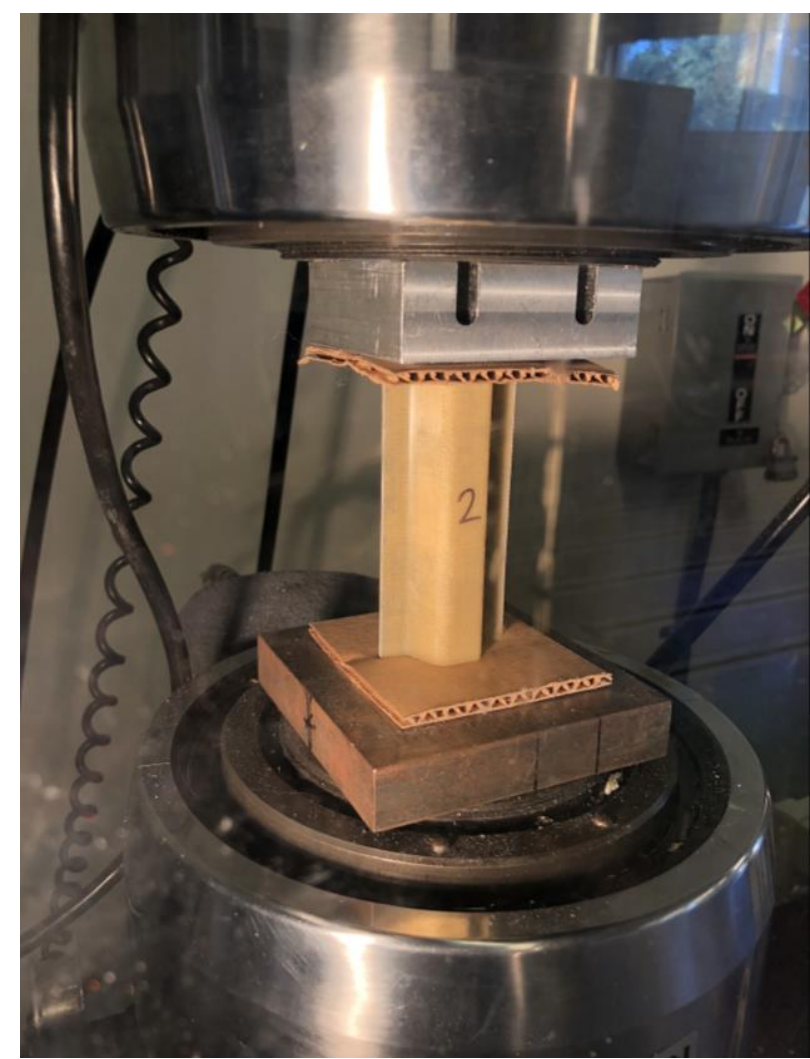

Figure 19: 4-Inch Long Hemp Sample Axial Compression Test

machine using the setup seen in Fig. 19. This setup involved sandwiching the test sample between two flat plates set over the tensile grips and two cardboard pieces, used to further offset unevenness and prevent slipping which would cause eccentric loading and unreliable results. The axial compression test was run at a constant compression speed of .005 inches per second. The test output the location of the head in inches and the force in pounds. 
In order to do this, the proper voltage relationships needed to be established in LabView and it was important to establish a proper and standard output format and naming convention. All the axial location data and converted readings from the force transducer were written to a text file which could be copied into excel for data processing and charting.

The progression of this test can be seen in Fig. 20 as the oneinch hemp sees local buckling to the point of a significantly reduced compression force and the test is stopped after post-yield stresses have dissipated.

Beginning with what was considered the control case in this research, the all-carbon fiber samples, all lengths from one to four inches were tested for each case. Each test was conducted as similarly as possible, taking care to replace the cardboard when it was damaged and to preload each sample.

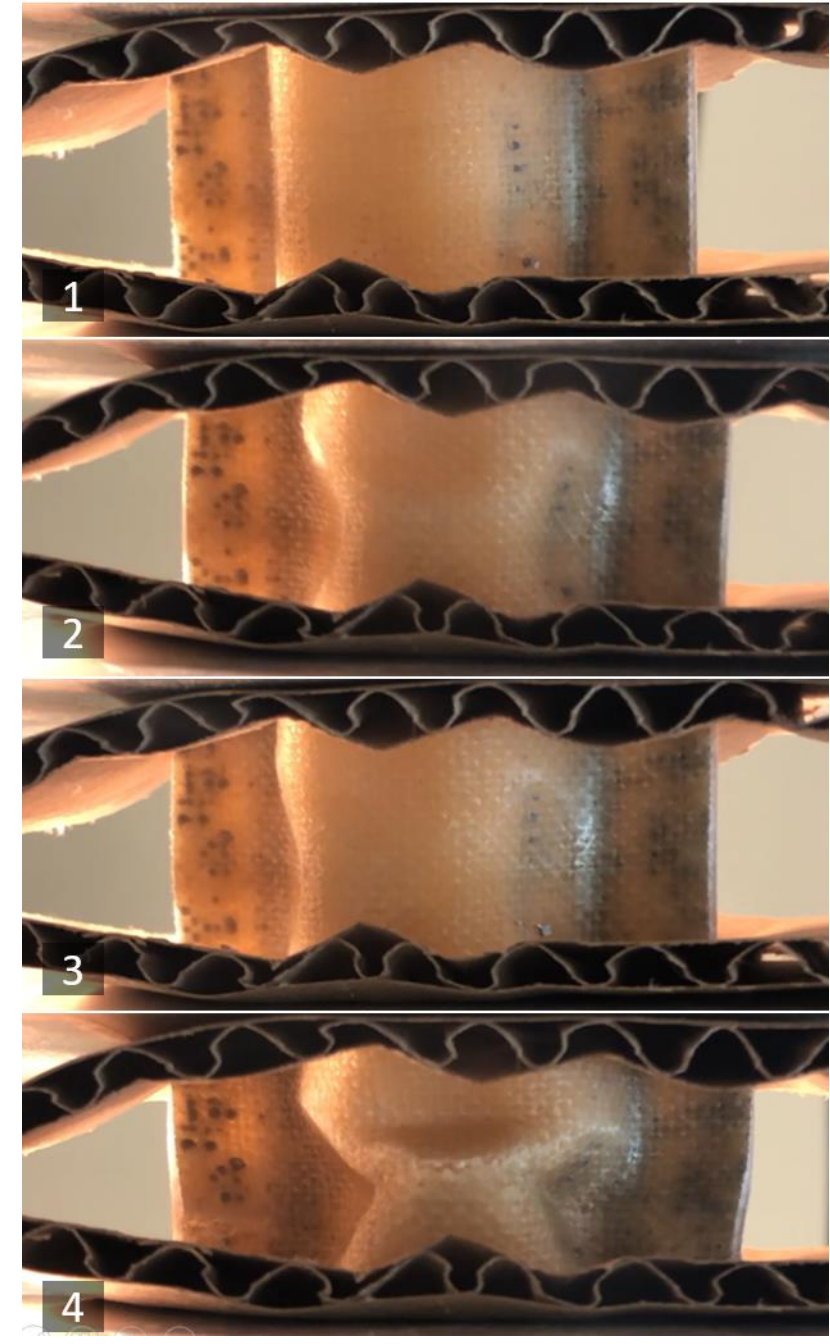

Figure 20: 1-Inch Hemp Compression Test Progression with Cardboard Buffer 


\section{EXPERIMENTAL RESULTS AND DISCUSSION}

\subsection{Carbon Fiber Results}

The first samples that were tested on the Instron 8801 under compression loading were the all-carbon fiber composite samples. These samples were set up as described previously, as straight as possible, using a cardboard buffer, and using a constant rate of compression. With the all-carbon fiber samples, it was expected to see some early failure due to debonding as was seen in previous research. However, the carbon fiber was also expected to be the stiffer and stronger of the two materials, being synthetic fibers compared to the organic fibers of the hemp.

\subsubsection{1-Inch Length}

The 1-inch all-carbon fiber samples were the first to be tested. Table 4 shows the ultimate loads sustained by each of the samples as well as the average ultimate load of 1114 pounds. Table 5 displays the ultimate stress sustained by each sample - calculated using the cross-sectional area - as well as the average ultimate stress of 14527 . This ultimate stress should be taken as an average of the stresses seen by the structure as a whole. 
Table 4: 1-Inch Carbon Fiber Ultimate Loads

\begin{tabular}{|r|r|r|r|r|r|}
\hline \multicolumn{5}{|c|}{ Ultimate Load (lbs) } \\
Sample 1 & Sample 2 & Sample 3 & Sample 4 & AVG & STDEV \\
\hline 1131 & 1072 & 933 & 1321 & 1114 & 160.92 \\
\hline
\end{tabular}

Table 5: 1-Inch Carbon Fiber Ultimate Stresses

\begin{tabular}{|r|r|r|r|r|}
\hline \multicolumn{5}{|c|}{ Ultimate Stress (lbs/in^2) } \\
\hline Sample 1 & Sample 2 & Sample 3 & Sample 4 & AVG \\
\hline 14690 & 13854 & 12139 & 17424 & 14527 \\
\hline
\end{tabular}

Fig. 21 shows the load-displacement curve for each of the 1-inch all-carbon fiber samples. Each of the 1-inch carbon fiber samples failed at roughly the same load force but the post-failure stress behavior varied between each sample. Fig. 22 displays all-carbon fiber sample 2 during the compression test. While in other samples the first failure is clearly shown with the delamination of the flat plates on both sides this sample shows more gradual yielding as the edge splinters and buckles by the fourth picture. This, in addition to the high load sustained post-failure, suggests a bearing stress limit has been reached, due to the miniscule cross-section of the sample edges. 


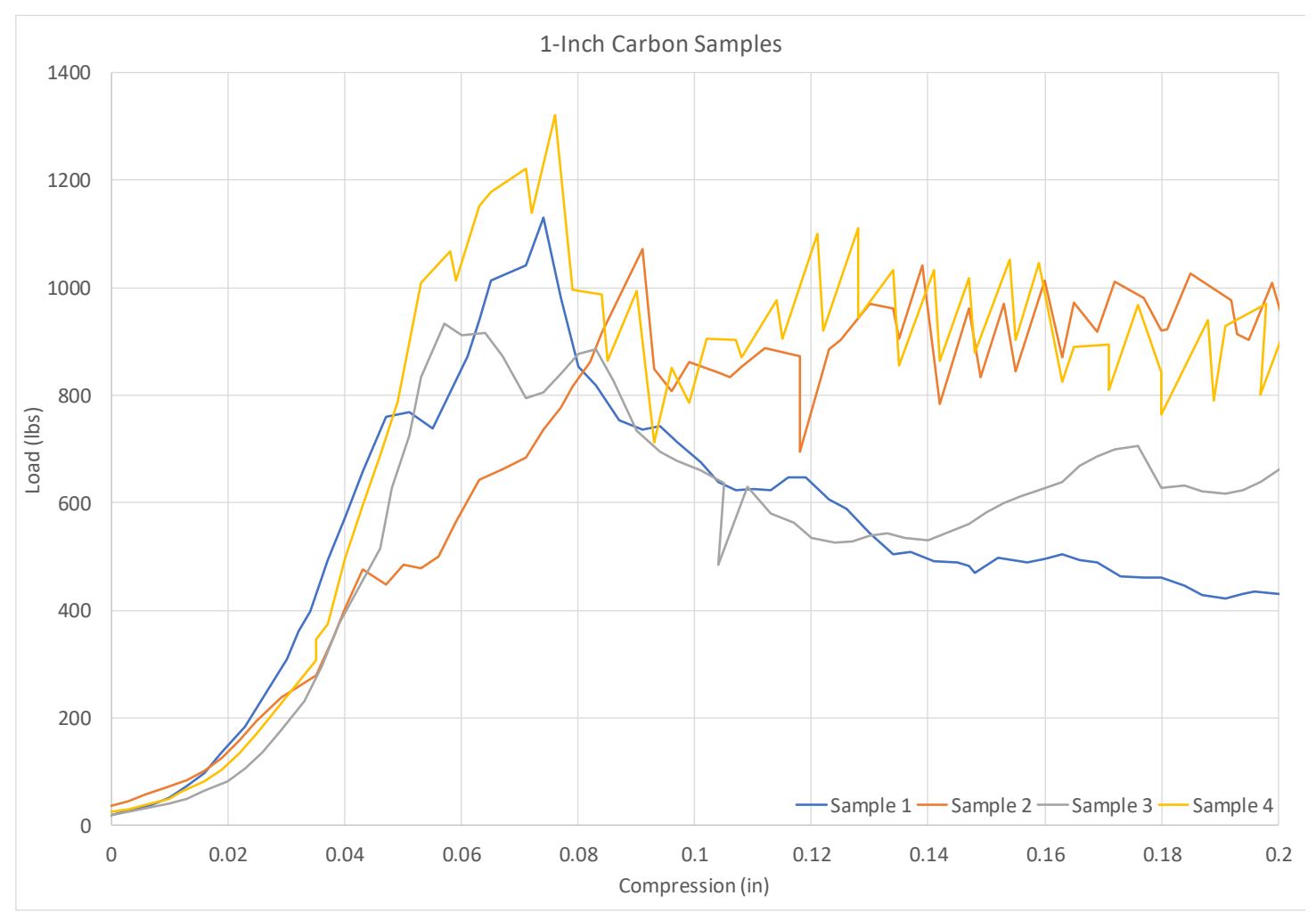

Figure 21: 1-Inch Carbon Fiber Load-Displacement Data

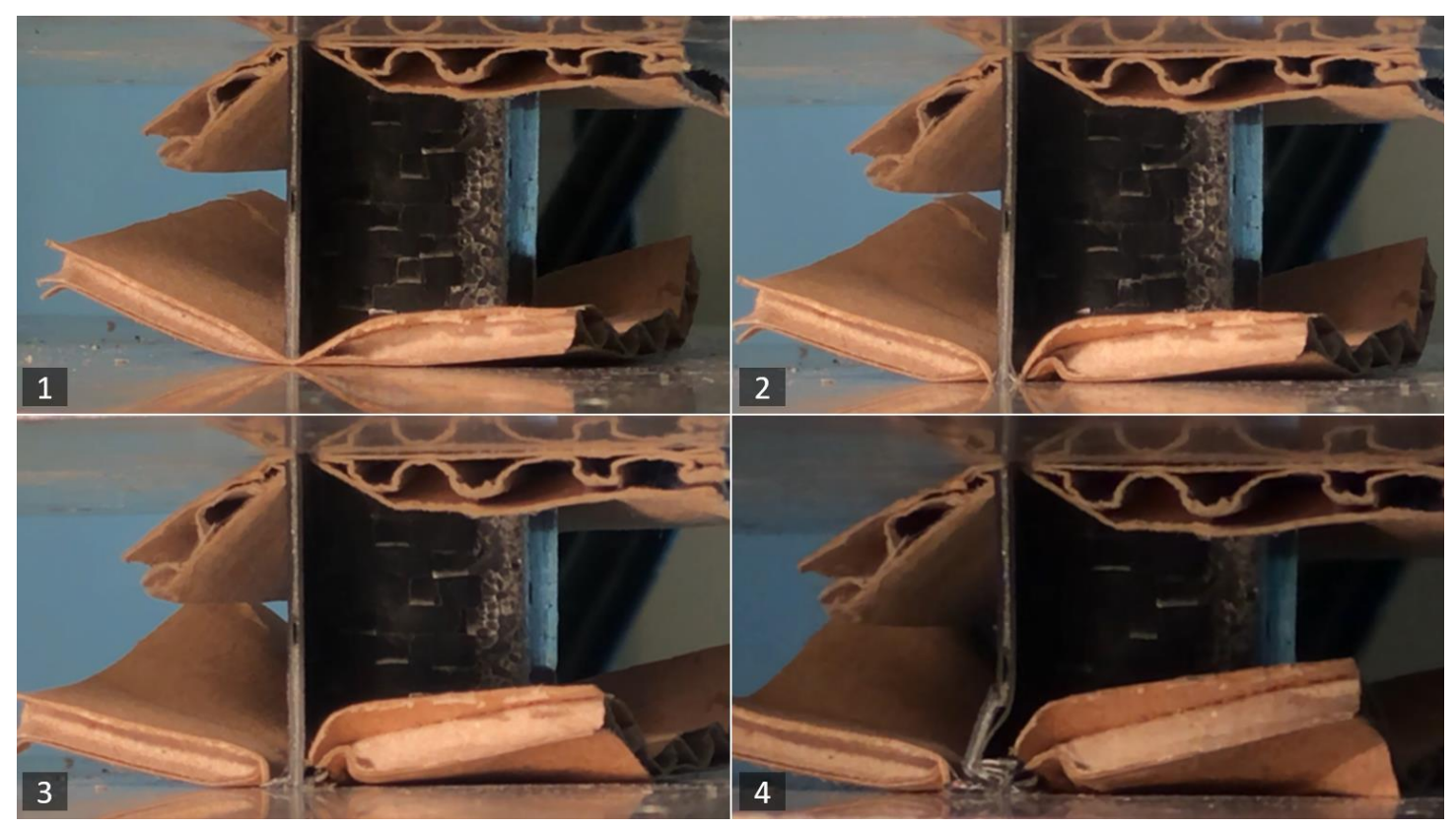

Figure 22: 1-Inch Carbon Fiber Compression Test with Edge Buckling 
All four of the samples fractured at around the same point. To analyze the stiffness of each composite, the linear elastic region of the load-compression graphs of each sample were examined. As it can be seen in Fig. 21, the linear elastic region of each sample occurs around the range of 300 to 800 pounds - roughly around $30 \%$ to $80 \%$ of the ultimate load.

These regions of the load-displacement curves can be seen in Fig. 23 as dotted lines, bounded by diamonds. These portions of the data were extracted and the slopes of points before first failure of each data set were used to calculate the stiffness for each sample. The average stiffness for the 1-inch all-carbon fiber samples was 29418 pounds per inch. As will be seen, this is low for all samples but especially low for the all-carbon fiber samples.

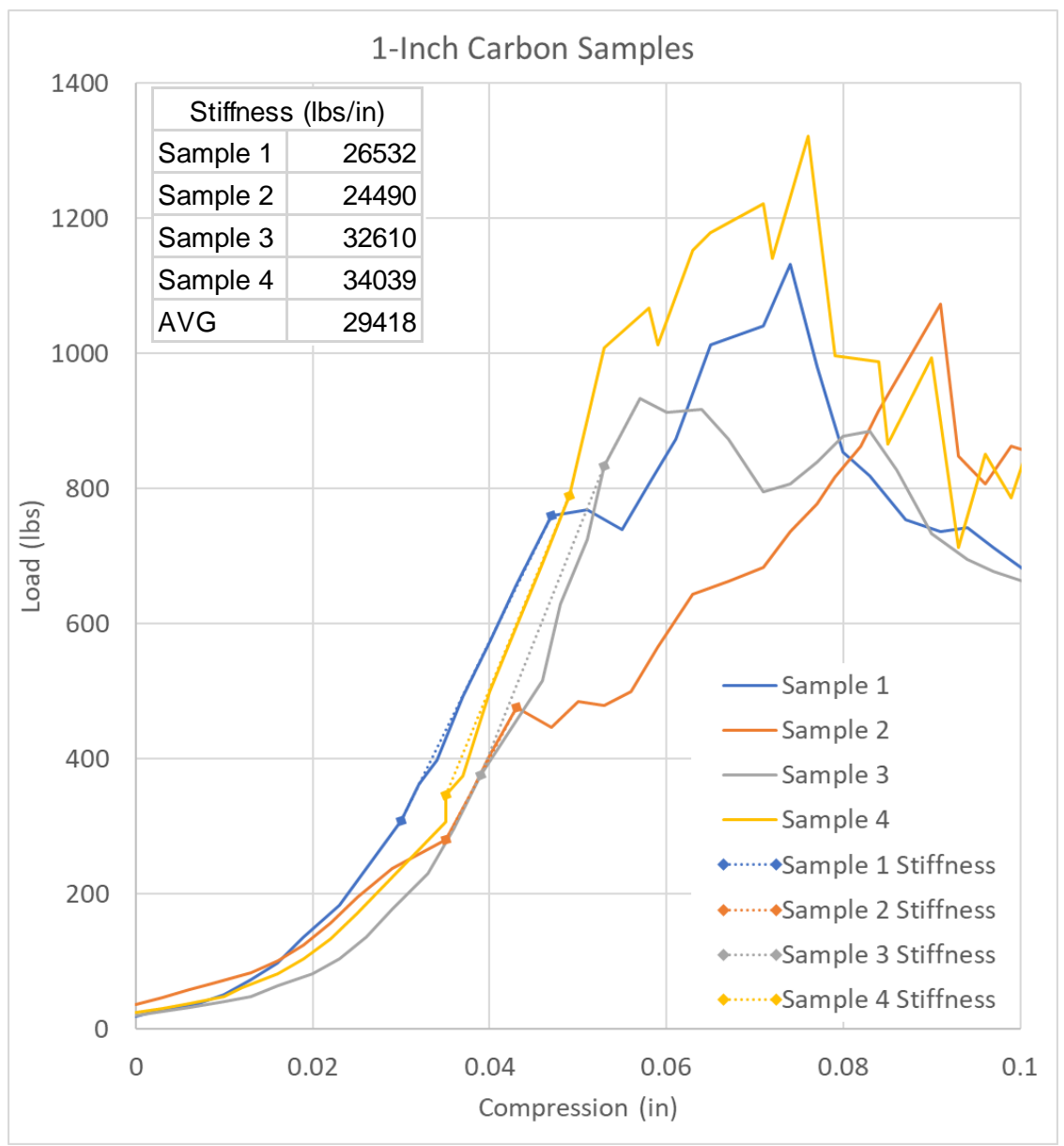

Figure 23: 1-Inch Carbon Fiber Stiffnesses 
Stiffness is used in this case because not only does the material affect the test results, but largely the structure does as well. The stiffness, $\mathrm{S}$, of a structure is represented by the equation below where $\mathrm{F}$ is the load applied and $\Delta \mathrm{L}$ is the change in the length of the sample through compression.

$$
\text { (2) } \quad \mathrm{S}=\mathrm{F} / \Delta \mathrm{L}
$$

\subsubsection{2-Inch Length}

Next, the 2-inch carbon fiber composite samples were tested. Fig. 24 displays the results of the constant compression rate test the 1-inch carbon fiber samples experienced. It displays 2 distinct failure modes for samples 1 but only one failure mode for sample 2 . Again, it is believed that the first sample failed in bonding first before ultimately failing due to the axial stress. The average ultimate load that the samples held was 1504 pounds, seen in Table 6. Based on the cross-sectional area of the sample, Table 7 lists the ultimate stress each sample held before failures and the 2 loads at which each failure occurred. The average ultimate stress that the samples held was 19479 pounds per square inch.

Table 6: 2-Inch Carbon Fiber Ultimate Loads

\begin{tabular}{|c|c|c|c|}
\hline \multicolumn{4}{|c|}{ Ultimate Load (lbs) } \\
Sample 1 & Sample 2 & AVG & STDEV \\
1543 & 1464 & 1504 & 55.6748 \\
\hline
\end{tabular}

Table 7: 2-Inch Carbon Fiber Ultimate Stresses

\begin{tabular}{|r|r|r|}
\hline \multicolumn{3}{|c|}{ Ultimate Stress (lbs/in`2) } \\
Sample 1 & Sample 2 & AVG \\
\hline 20041 & 18917 & 19479 \\
\hline
\end{tabular}




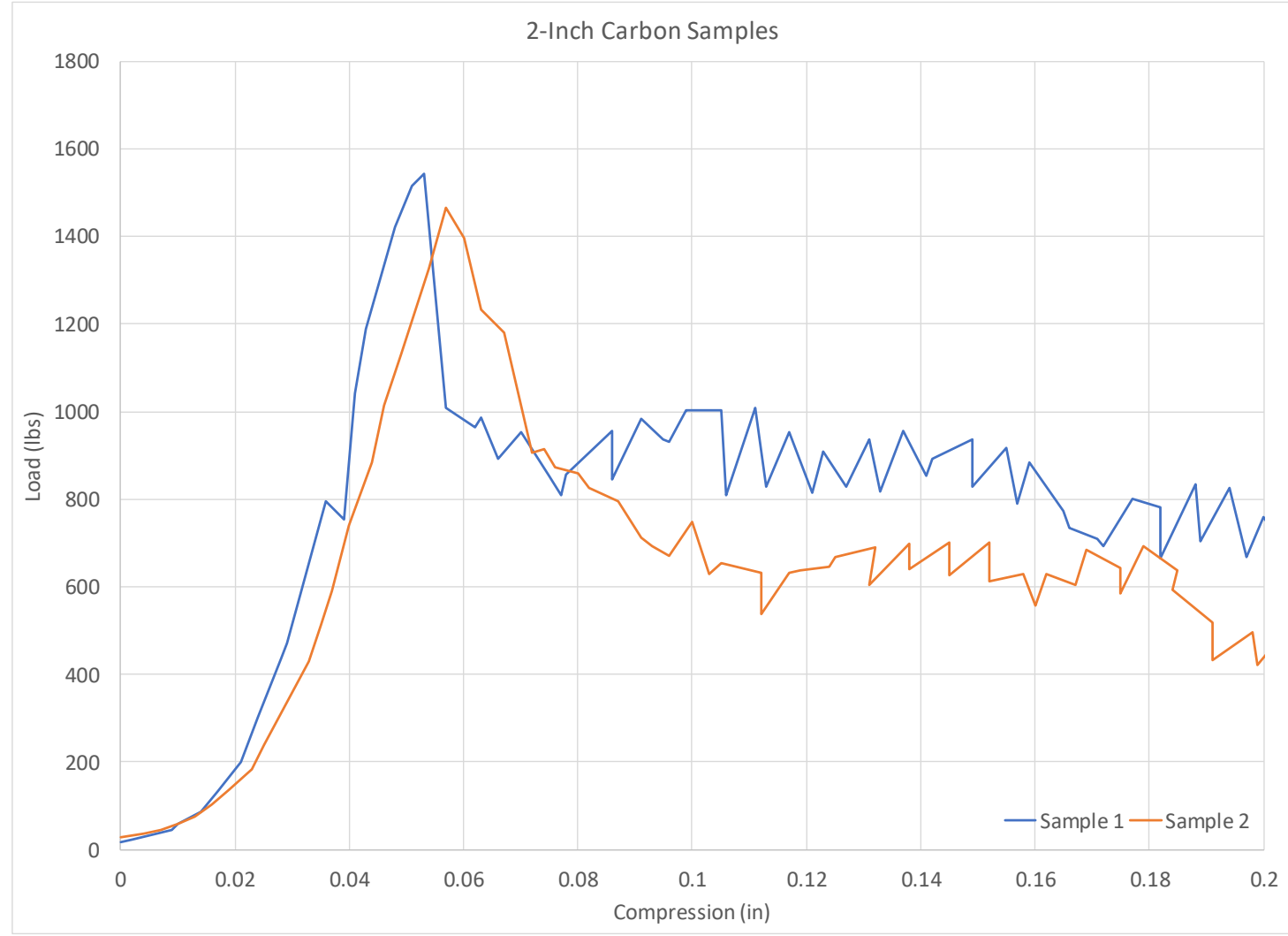

Figure 24: 2-Inch Carbon Fiber Load-Displacement Data

Each of the 2-inch carbon

fiber samples failed at around the same load force with similar post failure behavior. However, Fig. 24 displays differences in failure modes between samples 1 and 2 . Sample 1 had two failure modes while sample 2 only had one. Ultimate failure for both occurred at 1543 and 1464 pounds which were both hundreds of pounds more than

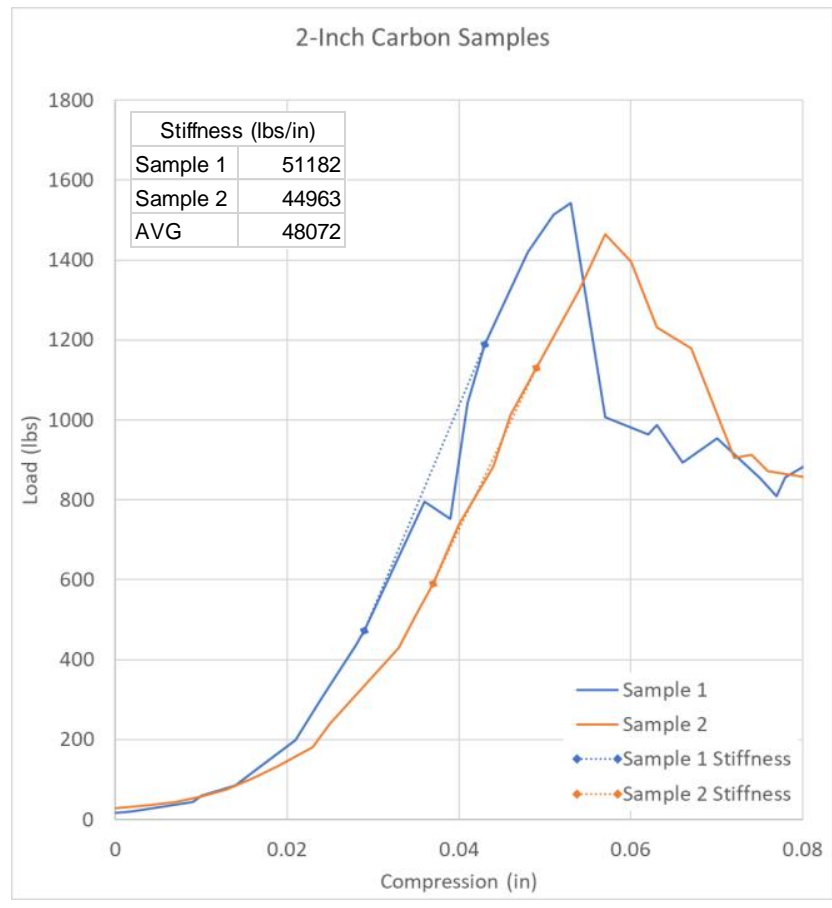

Figure 25: 2-Inch Carbon Fiber Stiffnesses 
the 1-inch carbon fiber samples. This can be attributed to the lack of debonding failure mode in these longer samples.

The stiffness increased greatly as well, with an average of 48072 pounds per inch. Again, one can see the slope of the linear region in Fig. 25 as well as the two calculated slopes for each sample. These slopes were taken after the load had ramped to a linear slope and before any leveling off. The distinctly higher ultimate load and average stiffness suggest that the higher bonding strength of the 2-inch samples allowed a greater yield strength of the overall structure pointing towards low bonding strength as the likely culprit of early yield in the 1-inch samples. The added bonding surface area increased the bond strength, which rose above the axial compressive yield strength.

\subsubsection{3-Inch Length}

Seen in Table 8, the average ultimate load for the 3-inch carbon fiber samples was 1384 pounds. Again, from the cross-sectional area of the sample, the average ultimate yield stress was 17958 pounds per square inch, seen in Table 9. These were both again much higher than the 1-inch samples, although not as high as the 2-inch samples. This could be due to the added length having added material that could contain a flaw or added effects of slight misalignment causing eccentric loading.

Table 8: 3-Inch Carbon Fiber Ultimate Loads

\begin{tabular}{|r|r|r|r|r|}
\hline \multicolumn{5}{|c|}{ Ultimate Load (lbs) } \\
Sample 1 & Sample 2 & Sample 3 & AVG & STDEV \\
1259 & 1437 & 1456 & 1384 & 108.569 \\
\hline
\end{tabular}


Table 9: 3-Inch Carbon Fiber Ultimate Stresses

\begin{tabular}{|r|r|r|r|}
\hline \multicolumn{4}{|c|}{ Ultimate Stress (lbs/in^2) } \\
\hline Sample 1 & Sample 2 & Sample 3 & AVG \\
\hline 16355 & 18570 & 18948 & 17958 \\
\hline
\end{tabular}

Fig. 26, the load-displacement curves for the 3-inch carbon fiber samples, shows several different failure modes among the three samples. This is again thought to be due to the extra length of the material, which allows more combinations of failures to develop. All three samples, however, showed multiple peaks near the ultimate failure of each sample. This indicates a bearing stress limit was reached before the ultimate compressive strength of the structure was reached. This and all other failure modes will be discussed in further detail in a dedicated section later on.

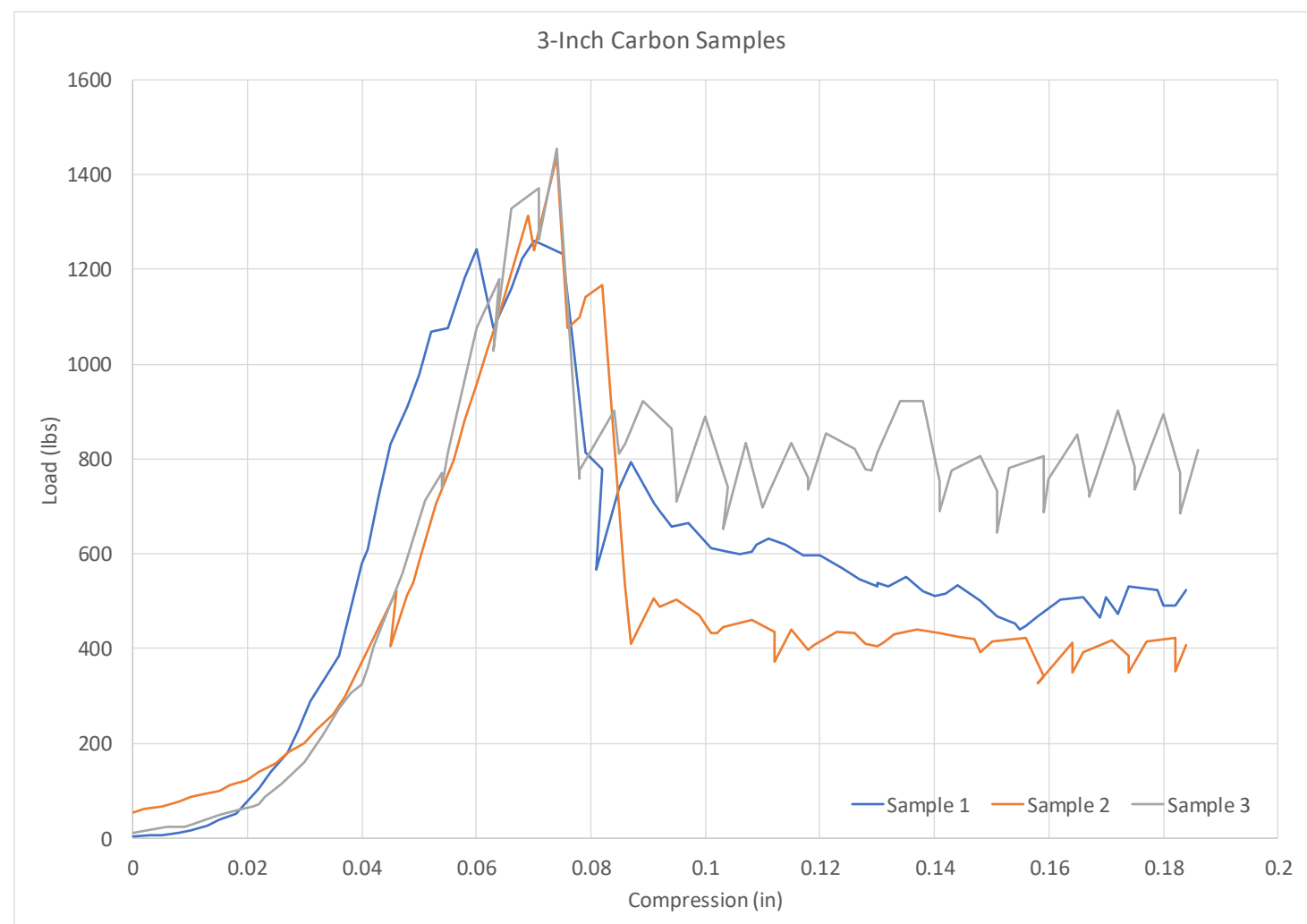

Figure 26: 3-Inch Carbon Fiber Load-Displacement Data 
All three samples failed in similar ways, along the top or the bottom. This can be seen in Fig. 27, where sample 1 failed along the top and samples 2 and 3 failed along the bottom. It is believed that this could be due to a slight angle introduced during the axial compression test by the cardboard used as a surface angle corrector.

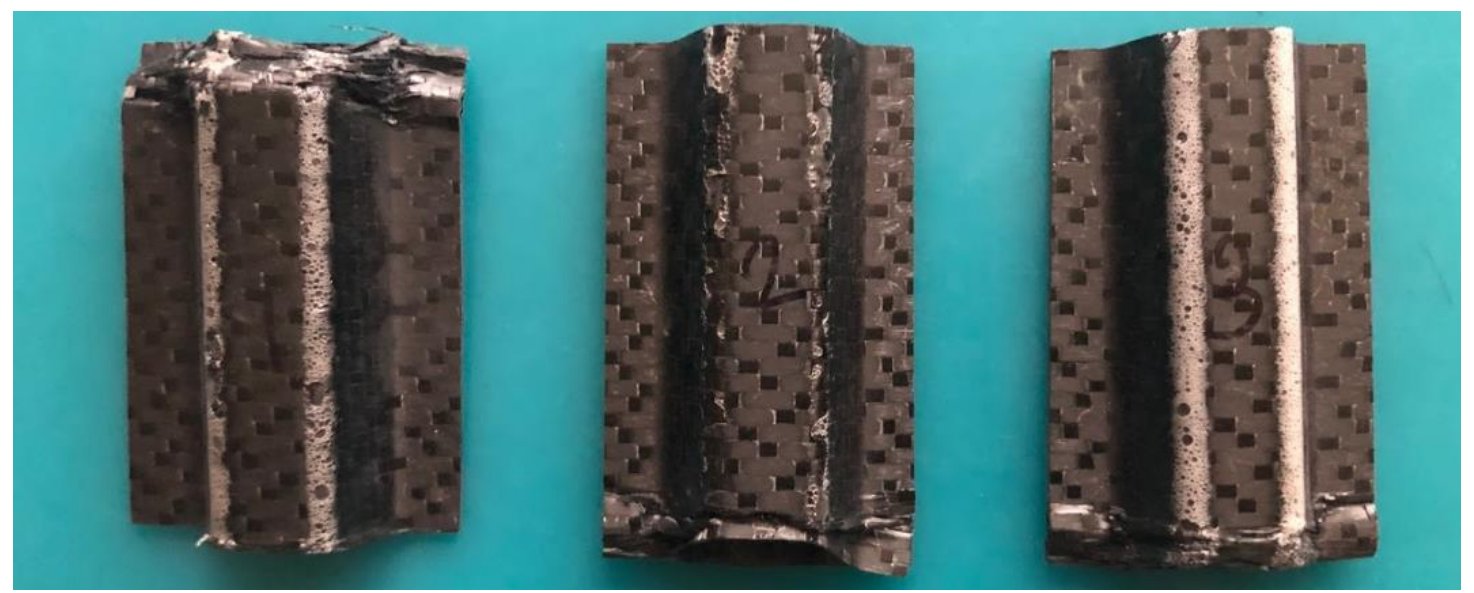

Figure 27: 3-Inch All-Carbon Fiber Compressed Samples

Fig. 28 shows the stiffness of the 3 -inch carbon fiber samples. The average stiffness of 39450 pounds per inch was comparable to that of the 2-inch carbon fiber samples and again much higher than that of the 1 inch samples. This again points to low bonding surface area and thus debonding being the cause of early failure in the 1-inch samples.

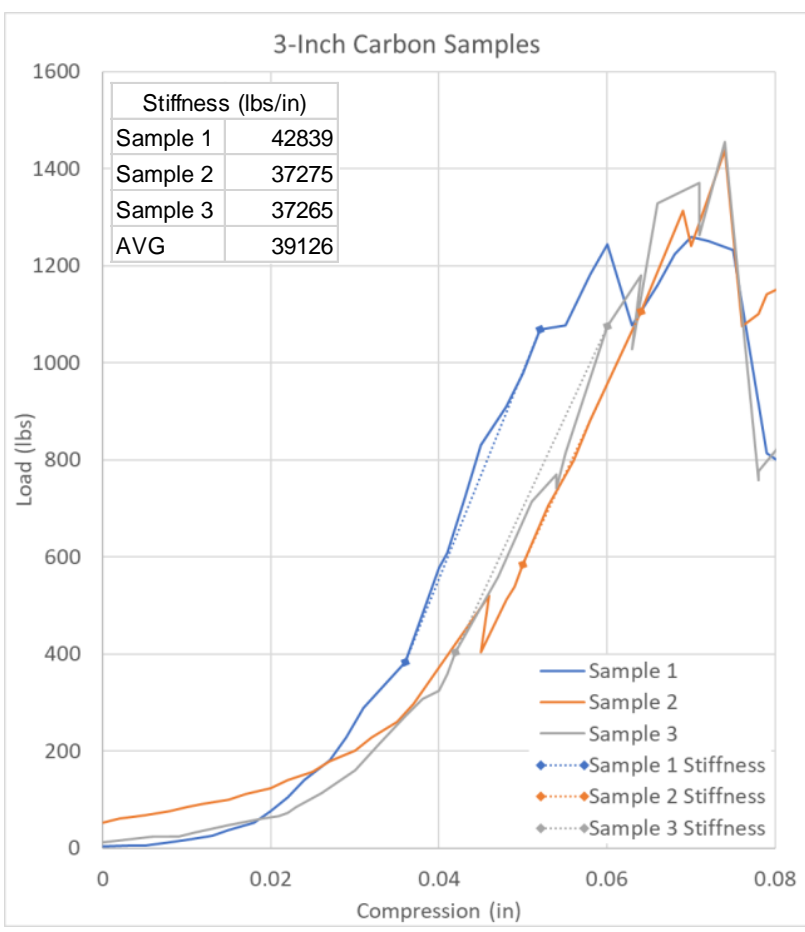

Figure 28: 3-Inch Carbon Fiber Stiffnesses 
Again, these stiffnesses were calculated using slope the linear portion of the loaddisplacement curves. The attempt was made to take these slopes at the section of the curve that was steepest, most linear, and independent of the differing failure modes, some of these slopes are very high and some are even negative. The continuous series of points was taken, however, to preserve experimental integrity.

\subsubsection{4-Inch Length}

Last of the fully carbon fiber samples to be tested were the 4-inch samples. Table 10 lists the ultimate load each sample held before failures. The average ultimate load that the samples held was 1363 pounds. Based on the cross-sectional area of the sample, Table 11 lists the ultimate stress each sample held. The average ultimate stress that the samples held was 17758 pounds per square inch.

Table 10: 4-Inch Carbon Fiber Ultimate Loads

\begin{tabular}{|r|r|r|r|r|r|}
\hline \multicolumn{5}{|c|}{ Ultimate Load (lbs) } & \\
Sample 1 & Sample 2 & Sample 3 & Sample 4 & AVG & STDEV \\
\hline 1585 & 1208 & 1290 & 1368 & 1363 & 162.057 \\
\hline
\end{tabular}

Table 11: 4-Inch Carbon Fiber Ultimate Stresses Ultimate Stress (lbs/in^2)

Sample 1 Sample 2 Sample 3 Sample 4 AVG

\begin{tabular}{ll|l|l|l|}
20588 & 15605 & 16787 & 18052 & 17758 \\
\hline
\end{tabular}




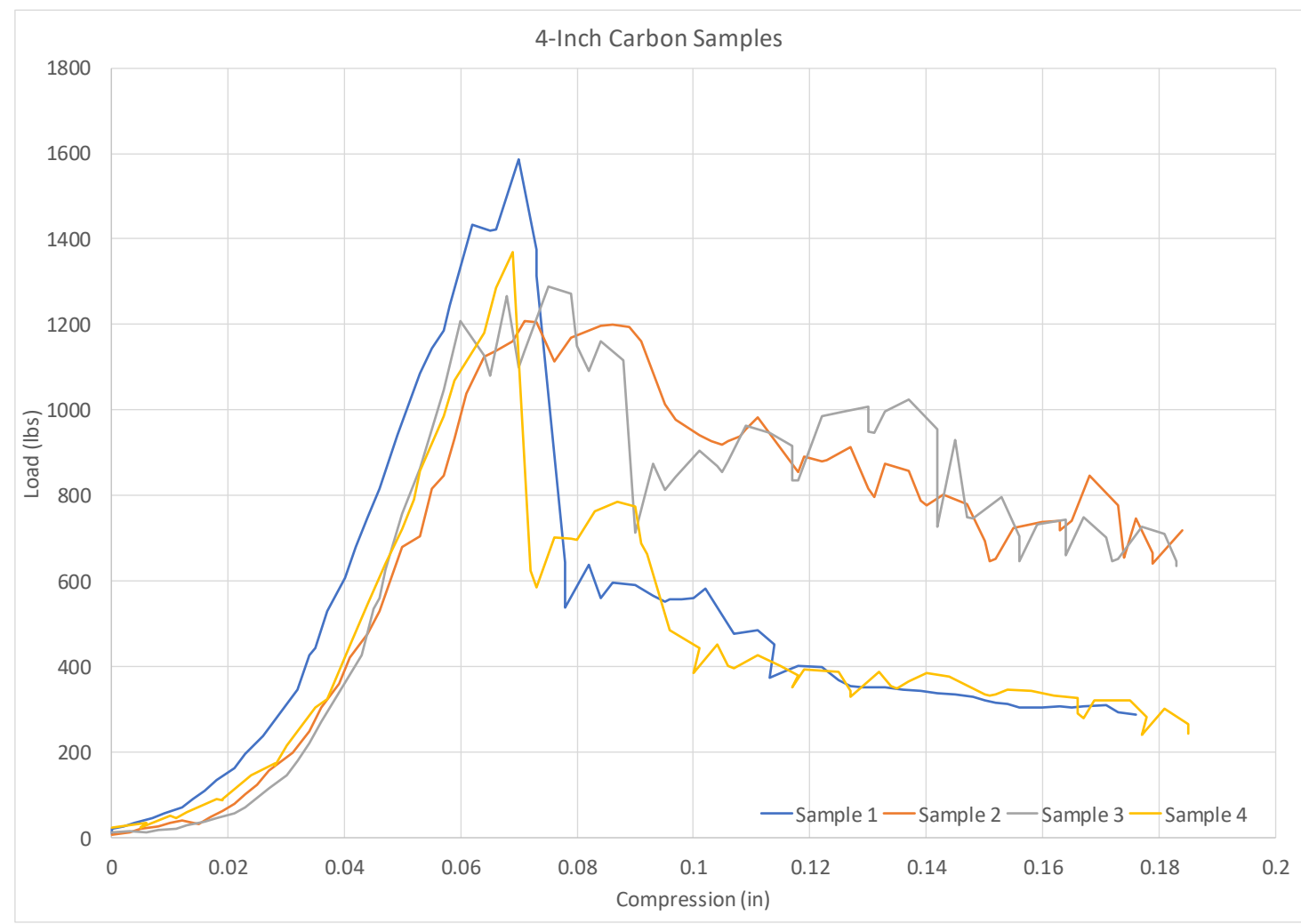

Figure 29: 4-Inch Carbon Fiber Load-Displacement Data

The load-displacement curves for the 4-inch carbon fiber samples, shown above in Fig. 29, display similar failure modes among the four samples through the linear portion of the constant rate axial compression. All samples except sample 4 showed multiple peaks near the ultimate failure of each sample. Again, this is thought to be due to buckling behavior at the top or bottom of the sample as a new contact surface is created and loaded.

Seen in Fig. 30, the first two 4-inch samples failed along the top surface while samples 3 and 4 failed along the bottom edge. During the test, some buckling could be seen which could be attributed to the length of the sample and slight misalignment of the angle of the sample. This manifested itself as the crushing of the front or back of the sample first. 


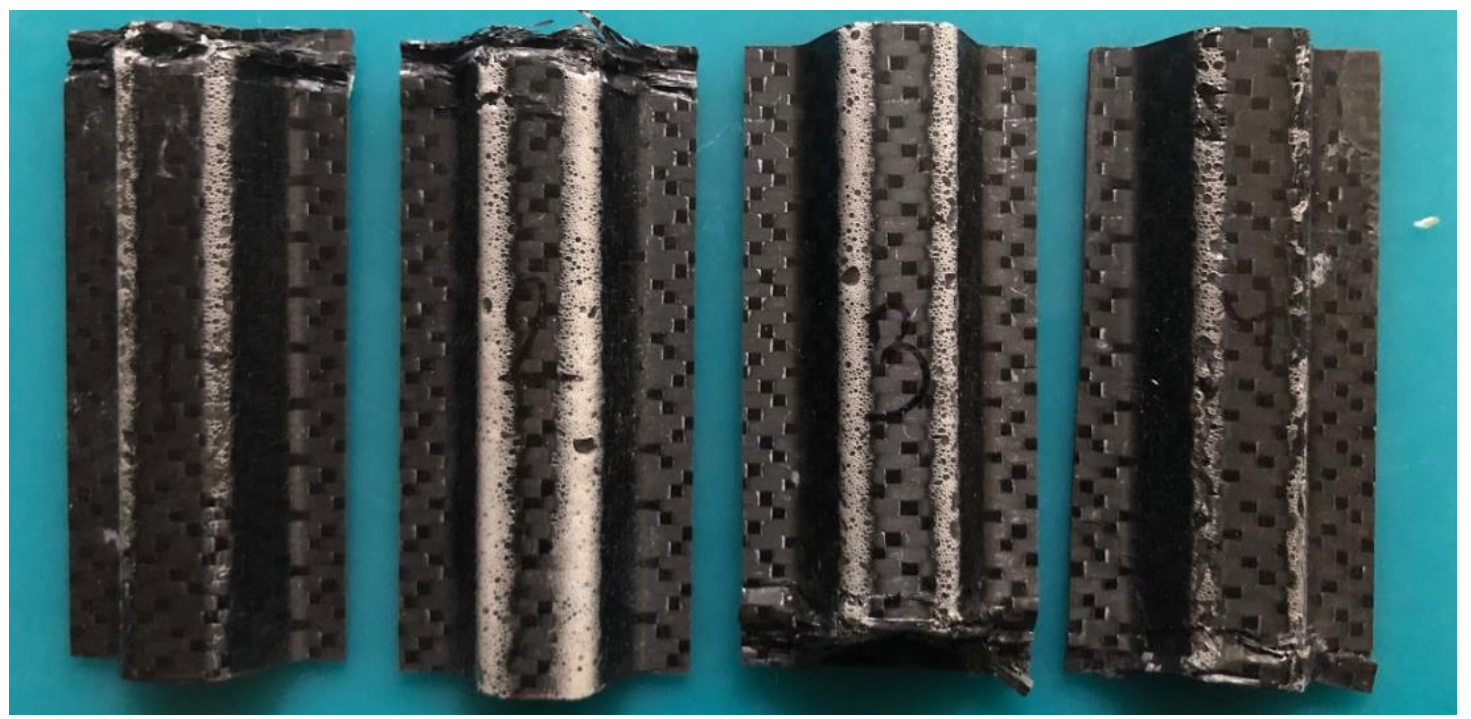

Figure 30: 4-Inch Carbon Fiber Samples after Axial Compression Test

The stiffnesses of the 4inch samples was comparable to that of the 3- and 2-inch samples, further supporting the bonding surface area as the cause for the early failure of the 1-inch samples. The average stiffness of these samples was 37043 pounds per inch, shown in Fig. 31. Buckling behavior is believed to have contributed to the diminished stiffness of the

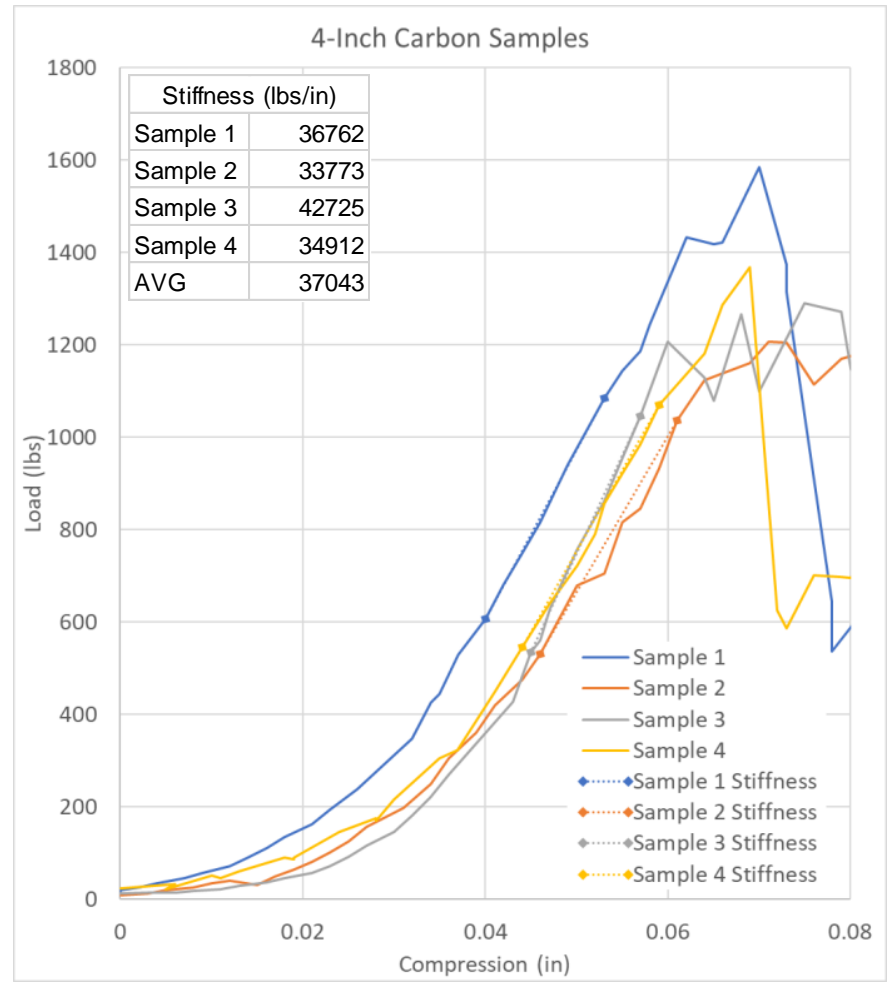

Figure 31: 4-Inch Carbon Fiber Stiffnesses 4-inch and 3-inch samples compared to the 2-inch samples. 
To summarize the all-carbon fiber results, the 1-inch samples had low bonding strength that resulted in the separation of core and skin, which then resulted in the corrugation taking on most of the load. The 3- and 4- inch samples had high bonding strength but succumbed to buckling failure modes, likely due to eccentric loading magnified by the length. The 2-inch samples had the highest mechanical properties due to the balance of high bonding strength and high enough resistance to buckling.

\subsection{Hemp Fiber Results}

The hemp fiber samples were tested using the exact same method as the carbon fiber samples, using the Instron 8801 uniaxial mechanical testing machine. As with the carbon fiber samples it was important to ensure flat surface along the top and bottom of each sample. However, it was also important to clean up the edges of any loose fiber and take care not to fray the edges during the cutting process. Any minimal remaining uneven material could be offset using the cardboard buffers.

\subsubsection{1-Inch Length}

Each of the 1-inch hemp fiber samples failed at around the same load force with very similar loading and post failure curves. Table 12 lists the ultimate load each sample held before failures. The average ultimate load that the samples held was 1344 pounds. Based on the cross-sectional area of the sample, Table 13 lists the ultimate stress each sample held. The average ultimate stress that the samples held was 7635 pounds per square inch. This ultimate stress was noticeably less than the carbon fiber samples due to the thicker cross section of the hemp fibers, despite having the same number of layers. 
Table 12: 1-Inch Hemp Fiber Ultimate Loads Ultimate Load (lbs)

Sample 1 Sample 2 Sample 3 Sample 4 Sample 5 AVG STDEV

\begin{tabular}{l|lllllll}
1425 & 1380 & 1396 & 1298 & 1223 & 1344 & 82.4297 \\
\hline
\end{tabular}

Table 13: 1-Inch Hemp Fiber Ultimate Stresses

\begin{tabular}{|r|rrrrr|}
\hline \multicolumn{7}{|c|}{ Ultimate Stress (lbs/in^2) } \\
\hline Sample 1 & Sample 2 & Sample 3 & Sample 4 & Sample 5 & AVG \\
\hline 8328 & 7945 & 7848 & 7422 & 6630 & 7635 \\
\hline
\end{tabular}

Fig. 32 shows the compression of the 1-inch hemp fiber samples. This is much different than the previously seen carbon fiber samples as the top and bottom surfaces remain aligned as the faces individually buckle. The load-displacement curves for the 1inch hemp fiber samples, seen in Fig. 33, display similar failure modes among the hemp fiber samples through the linear portion of the constant rate axial compression.

Each sample showed consistent stiffness slopes throughout the pseudo-linear region of the curve. This is thought to be due to the lack of debonding failure and the material being less brittle than the carbon fiber. This allows the material to sustain higher strains while warping and buckling instead of debonding. This buckling behavior at the face instead of the edge can be seen in Fig. 32. 

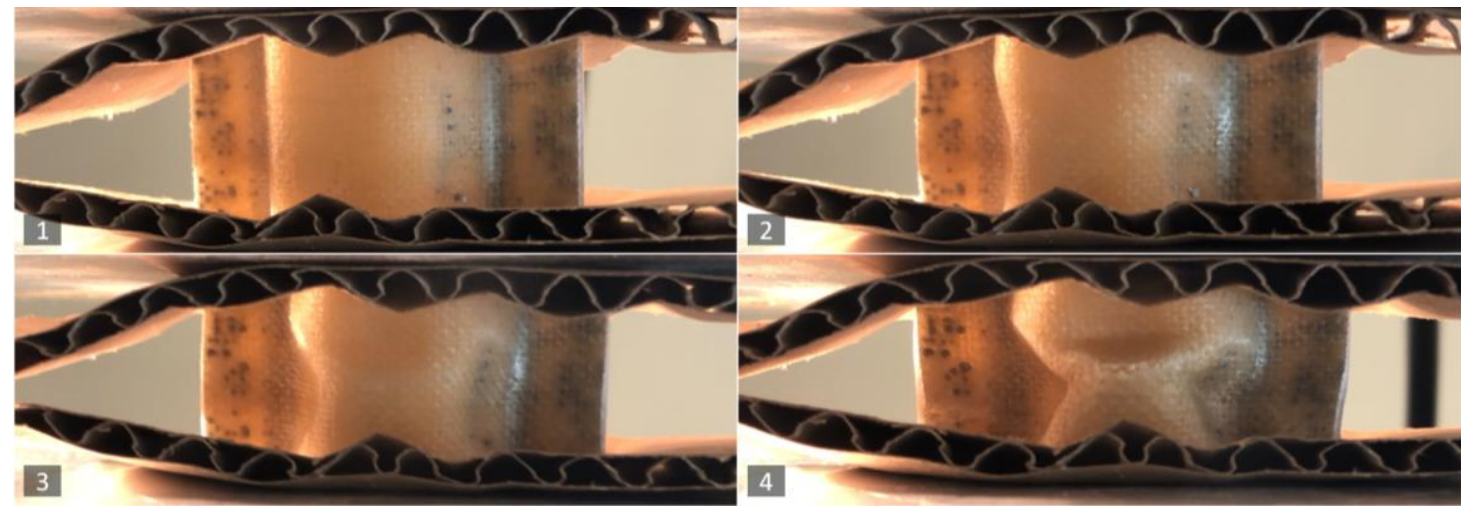

Figure 32: 1-Inch Hemp Fiber Compression Test with Front Face Buckling

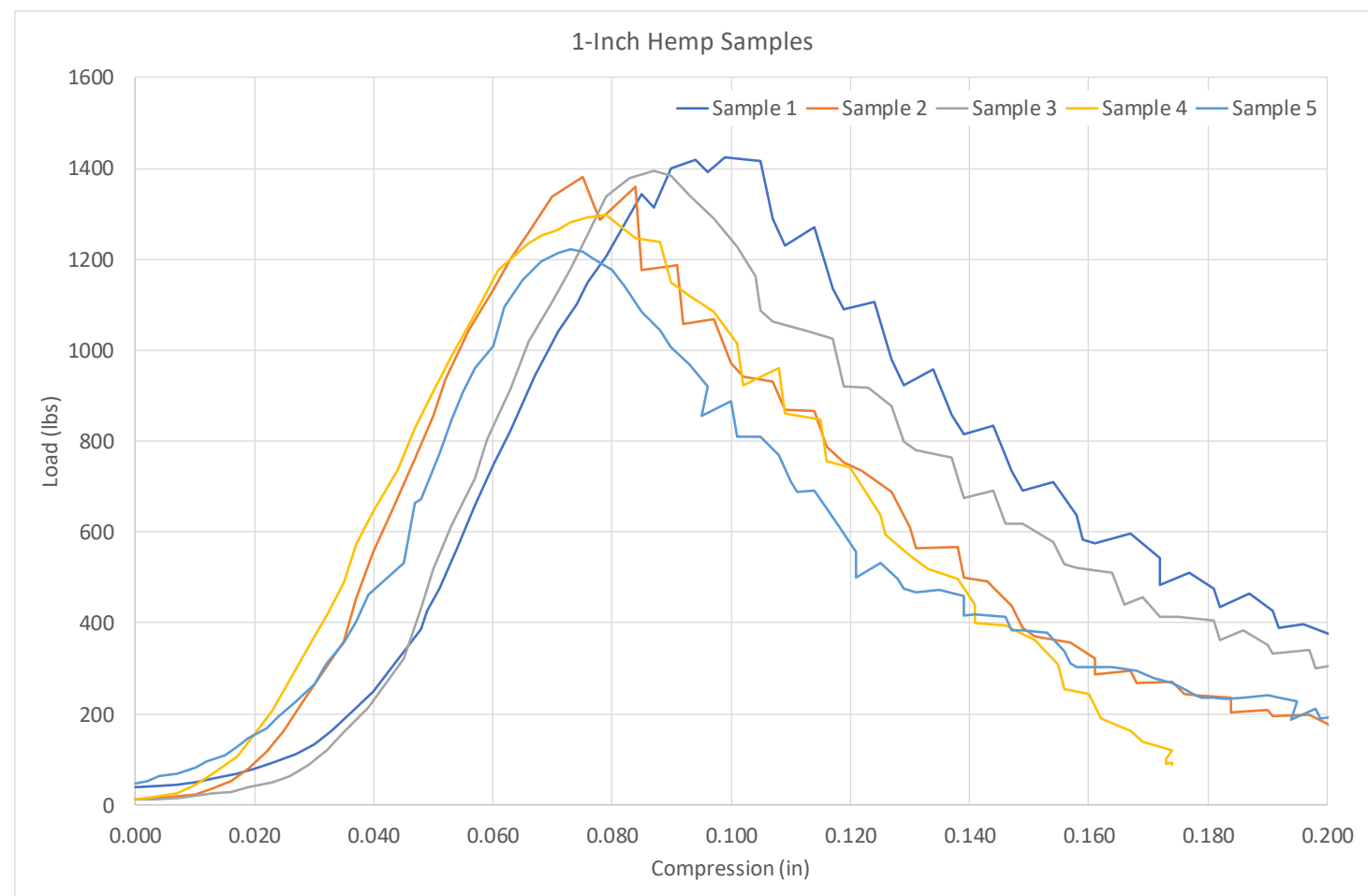

Figure 33: 1-Inch Hemp Fiber Load-Displacement Data 
To analyze the stiffness of each hemp fiber sample the same method of taking a slope from the most linear region of each curve around $30 \%$ to $80 \%$ of the ultimate load. The average stiffness of 28002 pounds per inch was lower than that of any of the all-carbon fiber samples, although not much lower than the 1-inch samples. The hemp material was thought of to be

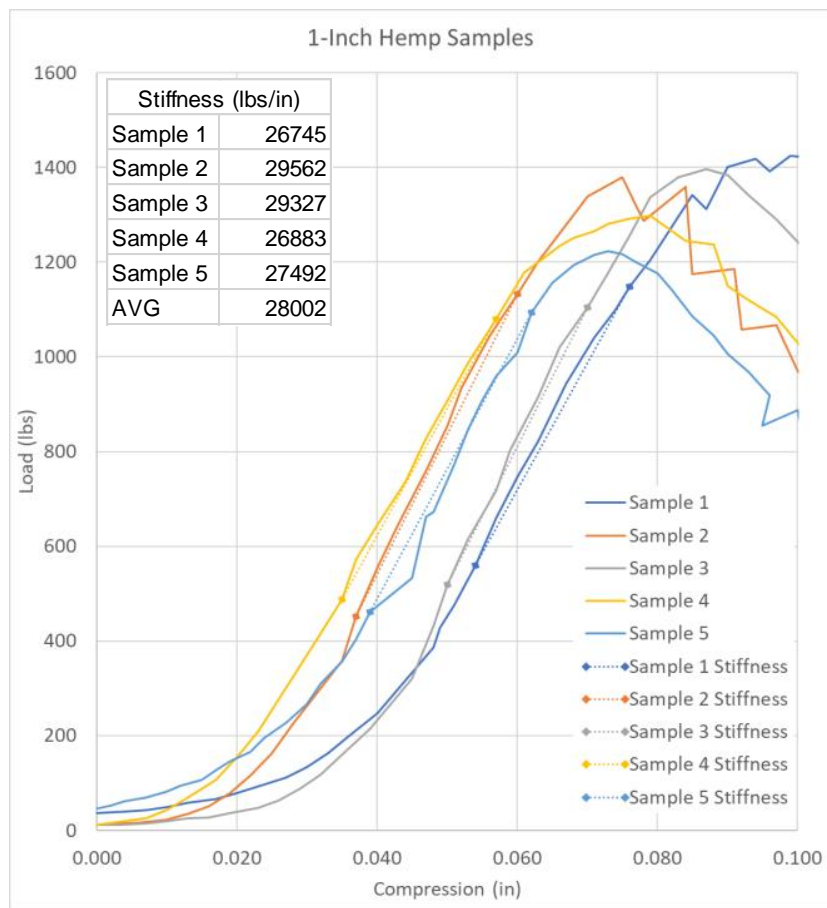

Figure 34: 1-Inch Hemp Fiber Stiffnesses

much less stiff than the carbon fiber. This again points to low bonding surface area being the cause of early failure in the 1 -inch carbon fiber samples. Compared to the 1 -inch carbon fiber stiffnesses, the hemp fiber stiffnesses were more consistent due to the smoother loading curves, seen in Fig. 34. This, again, can be attributed to the more ductile nature of the material.

It should be noted, however, that the stiffness does not account for the weight or density of the material. On average, the hemp and carbon fiber 1-inch samples weighed 3.12 and 1.95 grams respectively. The strength to weight ratio will be discussed further among the results of all testing. 


\subsubsection{2-Inch Length}

Next, the 2-inch hemp fiber composite samples were tested. Table 14 lists the ultimate load each sample held before failures and the 4 loads at which each failure occurred. The average ultimate load that the samples held was 1233 pounds. Based on the cross-sectional area of the sample, Table 15 lists the ultimate stress each sample held before failures and the 2 loads at which each failure occurred. The average ultimate stress that the samples held was 7071 pounds per square inch. These were both slightly less than the 1inch samples, despite the results being very consistent between samples. This could be attributed to the greater amount of material allowing more opportunity for failure. Samples 5 and 6 of this material configuration resulted in data capture error.

Table 14: 2-Inch Hemp Fiber Ultimate Loads

\begin{tabular}{|rrrrrr|}
\hline \multicolumn{5}{|c|}{ Ultimate Load (lbs) } \\
\hline Sample 1 & Sample 2 & Sample 3 & Sample 4 & AVG & STDEV \\
1162 & 1259 & 1284 & 1229 & 1233 & 52.5526 \\
\hline
\end{tabular}

Table 15: 2-Inch Hemp Fiber Ultimate Stresses

\begin{tabular}{|r|rrr|r|}
\hline \multicolumn{5}{|c|}{ Ultimate Stress (lbs/in^2) } \\
\hline Sample 1 & Sample 2 & Sample 3 & Sample 4 & AVG \\
6794 & 7250 & 7217 & 7025 & 7071 \\
\hline
\end{tabular}

The load-displacement curves for the 1-inch hemp fiber samples, seen in Fig. 35, once again display fairly consistent failure modes among the hemp fiber samples through the linear portion of the constant rate axial compression. However, the lower ultimate load strength is diminished. This is believed to be due to greater opportunity for buckling in the longer sample, seen in Fig. 36. 


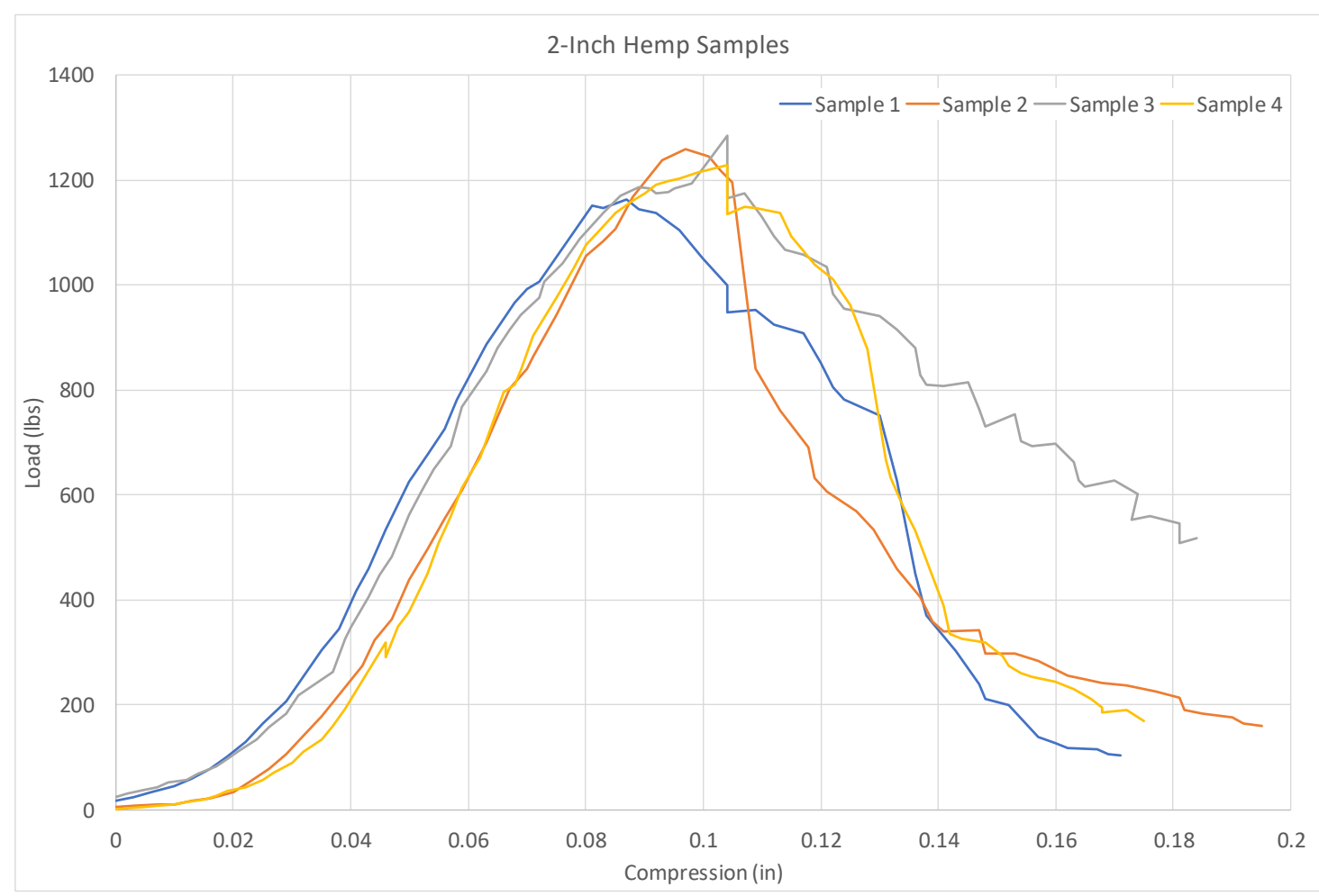

Figure 35: 2-Inch Hemp Fiber Load-Displacement Data

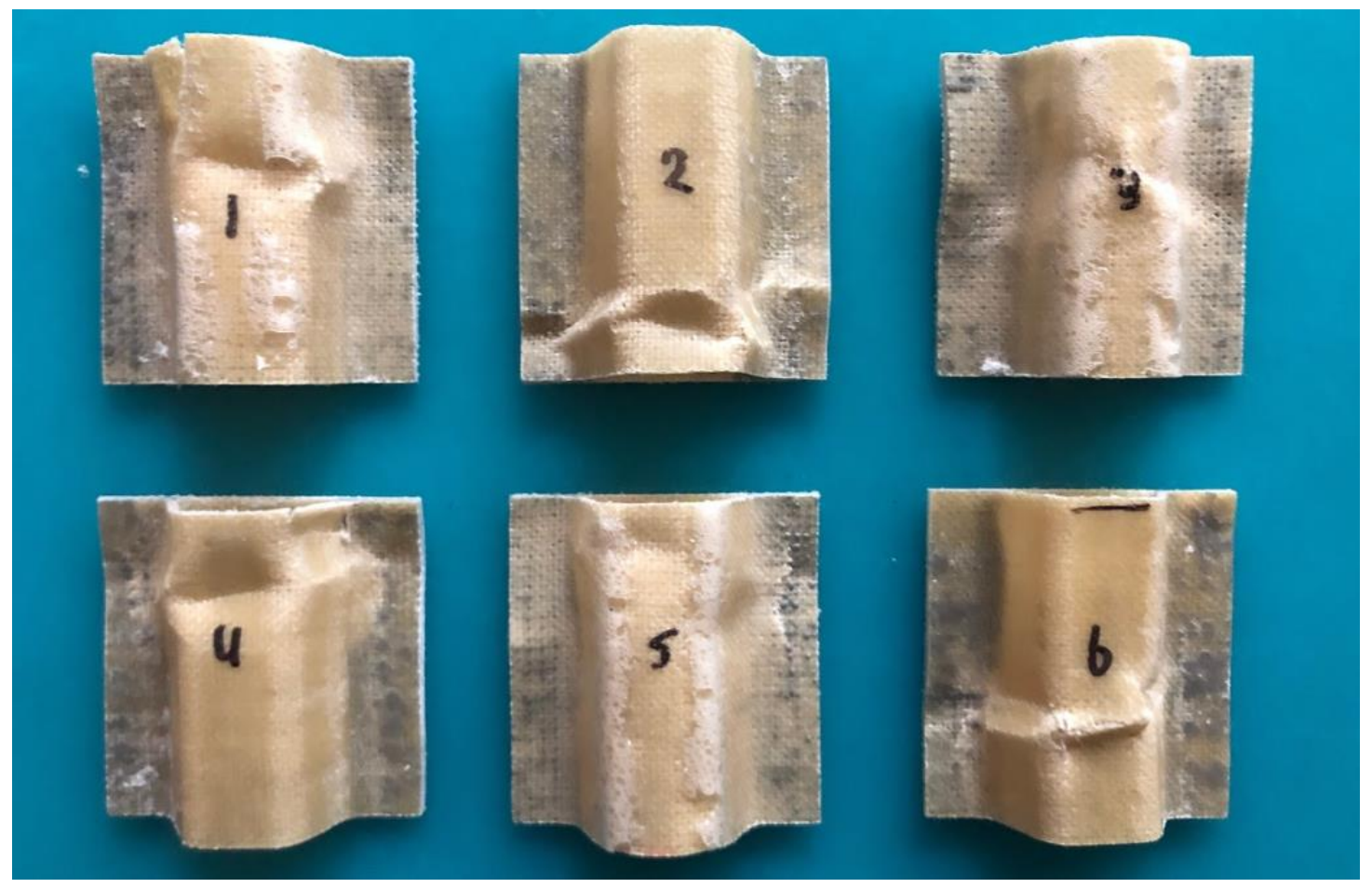

Figure 36: 2-Inch Hemp Fiber Failed Samples 
As seen in Fig. 36, each sample buckled at a different length along the sample face and buckling of the face never occurred along an even plane. This could be due to differences in resin concentration in the material, differences in bonding strength, or unevenness of the top or bottom edge of the sample. All of these will be discussed later as possible sources

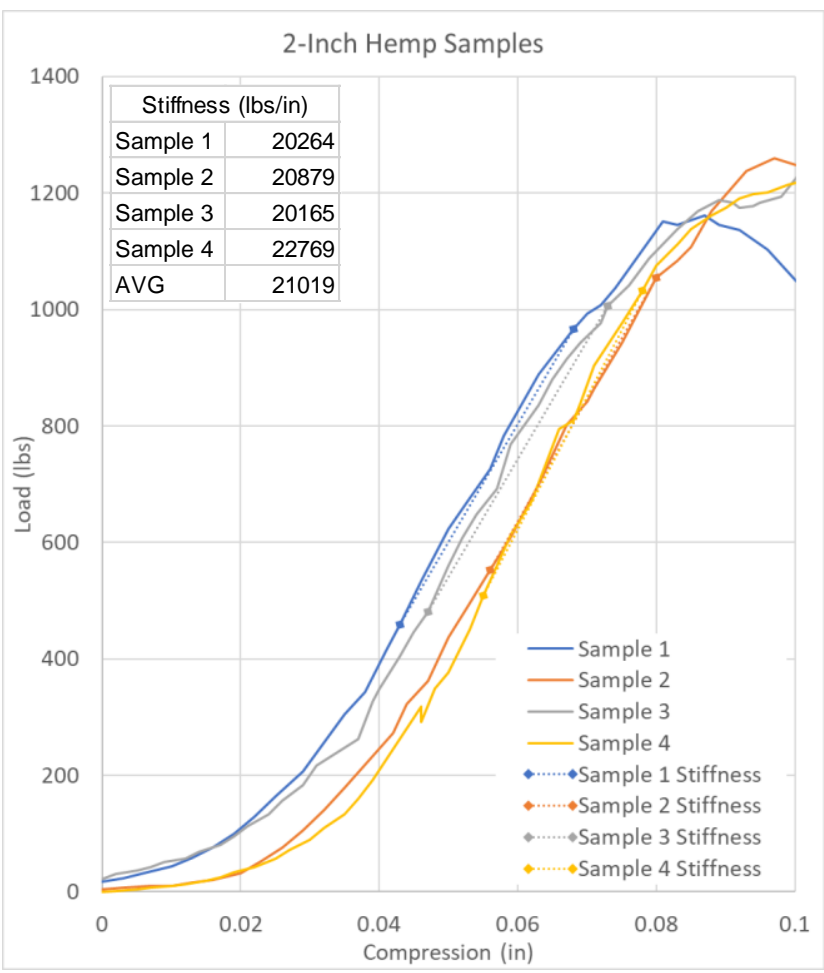

Figure 37: 2-Inch Hemp Fiber Stiffnesses of error. Fig. 37 displays the stiffnesses of the 2-inch hemp fiber samples. The average stiffness of 21019 pounds per inch was much less than that of the 1-inch hemp fiber samples. This directs the cause of the reduced stiffness to the increased length of material - in the same way that the stiffness and length of a spring are inversely proportional.

\subsubsection{3-Inch Length}

Each of the 3-inch hemp fiber samples failed at almost the same load force. Table 16 lists the ultimate load each sample held before failures. The average ultimate load that the samples held was 1202 pounds. Based on the cross-sectional area of the sample, Table 17 lists the ultimate stress each sample held. The average ultimate stress that the samples 
held was 6904 pounds per square inch. These ultimate loads and ultimate stresses were slightly lower than the 2-inch hemp fiber samples.

Table 16: 3-Inch Hemp Fiber Ultimate Loads

\begin{tabular}{|r|rrr|r|}
\hline \multicolumn{5}{|c|}{ Ultimate Load (lbs) } \\
Sample 1 & Sample 2 & Sample 3 AVG & STDEV \\
\hline 1212 & 1183 & 1212 & 1202 & 16.9146 \\
\hline
\end{tabular}

Table 17: 3-Inch Hemp Fiber Ultimate Stresses

\begin{tabular}{|c|c|c|c|}
\hline & ate Stre & Ibs/in' & \\
\hline Sample 1 & nple 2 & pple 3 & \\
\hline 7086 & 6810 & 6815 & 6904 \\
\hline
\end{tabular}

The load-displacement curves for the 3-inch hemp fiber samples, seen in Fig. 38, display similar failure modes among the hemp fiber samples through the linear portion of the constant rate axial compression until post failure behaviors begin to diverge. Again, each sample showed consistent stiffness slopes throughout the pseudo-linear region of the curve which seems to characterize the hemp fiber samples. This is thought to be due to the lack of debonding failure and the material being less brittle than the carbon fiber. This allows the material to sustain higher strains while warping and buckling instead of debonding. This buckling behavior at the face instead of the edge can be seen in Fig. 39 . 


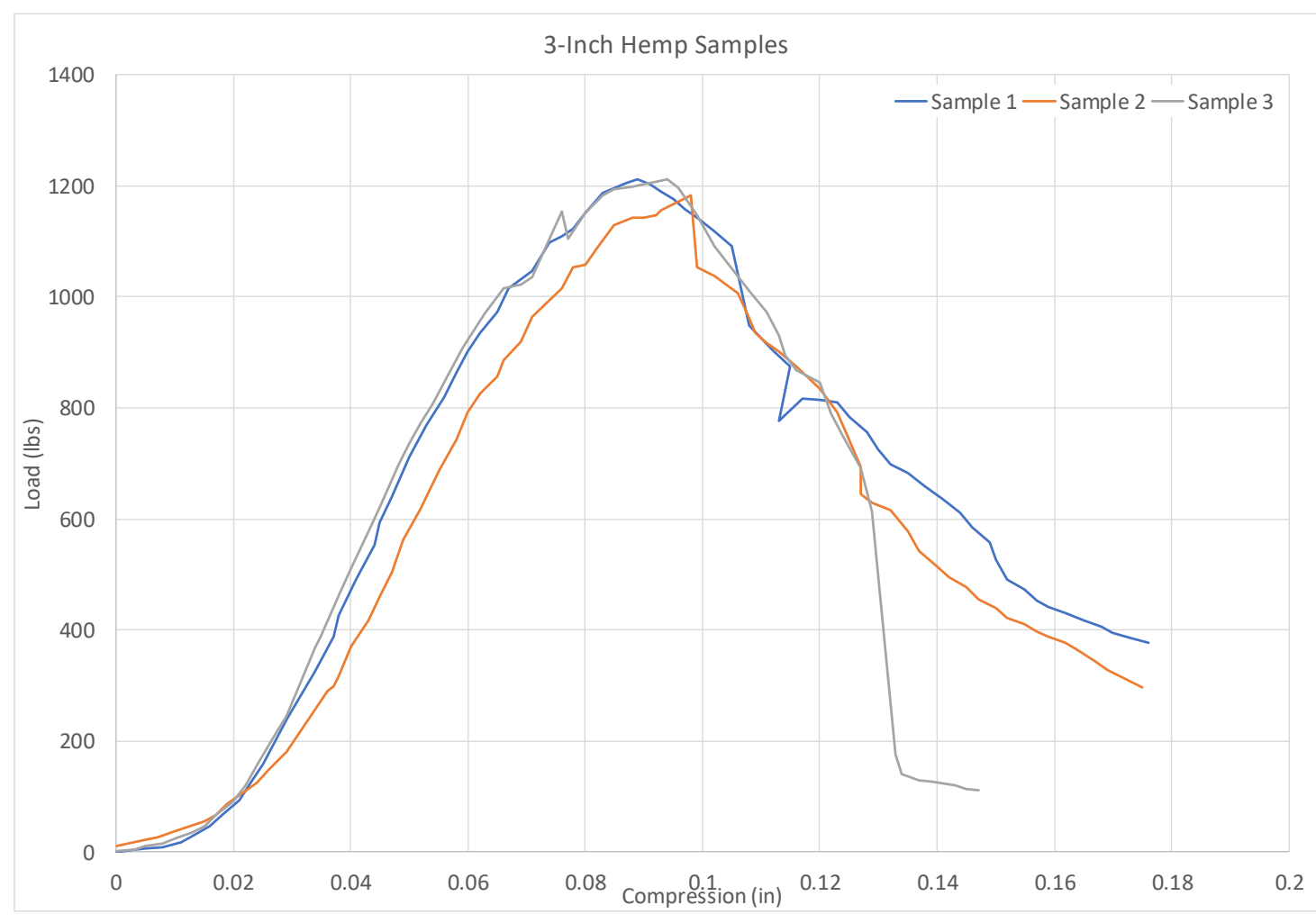

Figure 38: 3-Inch Hemp Fiber Load-Displacement Data

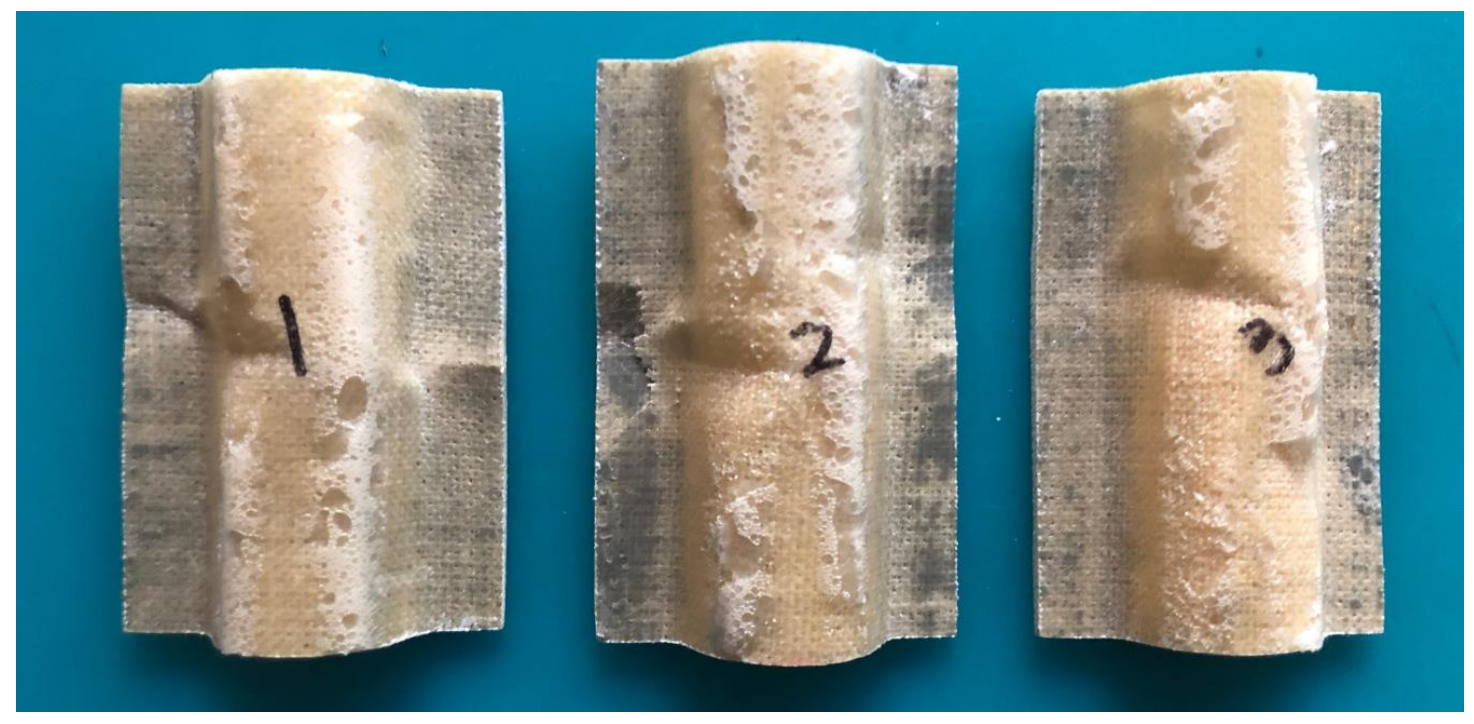

Figure 39: 3-Inch Hemp Fiber Compression Test with Front Face Buckling 
Fig. 40 displays the stiffnesses of the 3-inch hemp fiber samples. The average stiffness of 22090 pounds per inch was less than that of the 1-inch hemp fiber samples but slightly more than that of the 2-inch hemp fiber samples. The trend of decreasing stiffness with increasing length should continue but does not in this case. In the way that springs have

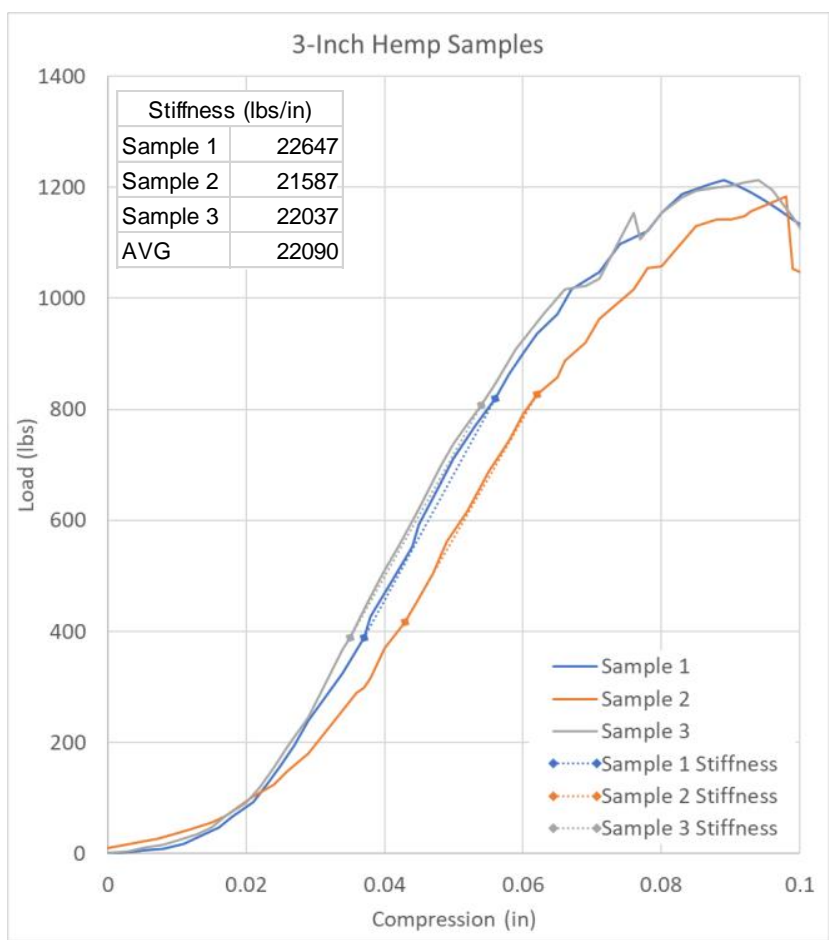

Figure 40: 3-Inch Hemp Fiber Stiffnesses diminished stiffness in series, 3-inch sample can be thought of as three 1-inch samples in series, resulting in diminished stiffness.

As seen in Fig. 39, each sample buckled around the middle of the front face of the sample. This supports the consistent stiffness and ultimate strength seen in this configuration, as well as the near-identical load displacement curves.

\subsubsection{4-Inch Length}

Last of the hemp fiber samples were the 4-inch samples. Table 18 lists the ultimate load each sample held before failures. The ultimate load that the sample held was 1162 pounds. Based on the cross-sectional area of the sample, Table 19 lists the ultimate stress each sample held. The ultimate stress that the sample held was 12527 pounds per square inch. 
Table 18: 4-Inch Hemp Fiber Ultimate

\begin{tabular}{|c|}
\hline Loads \\
\hline Ultimate Load (lbs) \\
\hline Sample 1 \\
\hline
\end{tabular}

Table 19: 4-Inch Hemp Fiber Ultimate Stresses

Ultimate Stress (lbs/in^2) Sample 1

The load-displacement curve for the 4-inch hemp fiber sample, seen in Fig. 41, again displays a consistent stiffness slope throughout the pseudo-linear region of the curve. The ultimate load and ultimate stress for this sample were lower than all the other hemp fiber samples. As seen in Fig. 42, this is due to the ductile nature of the material and the susceptibility of this asymmetric structure to buckling at longer lengths.

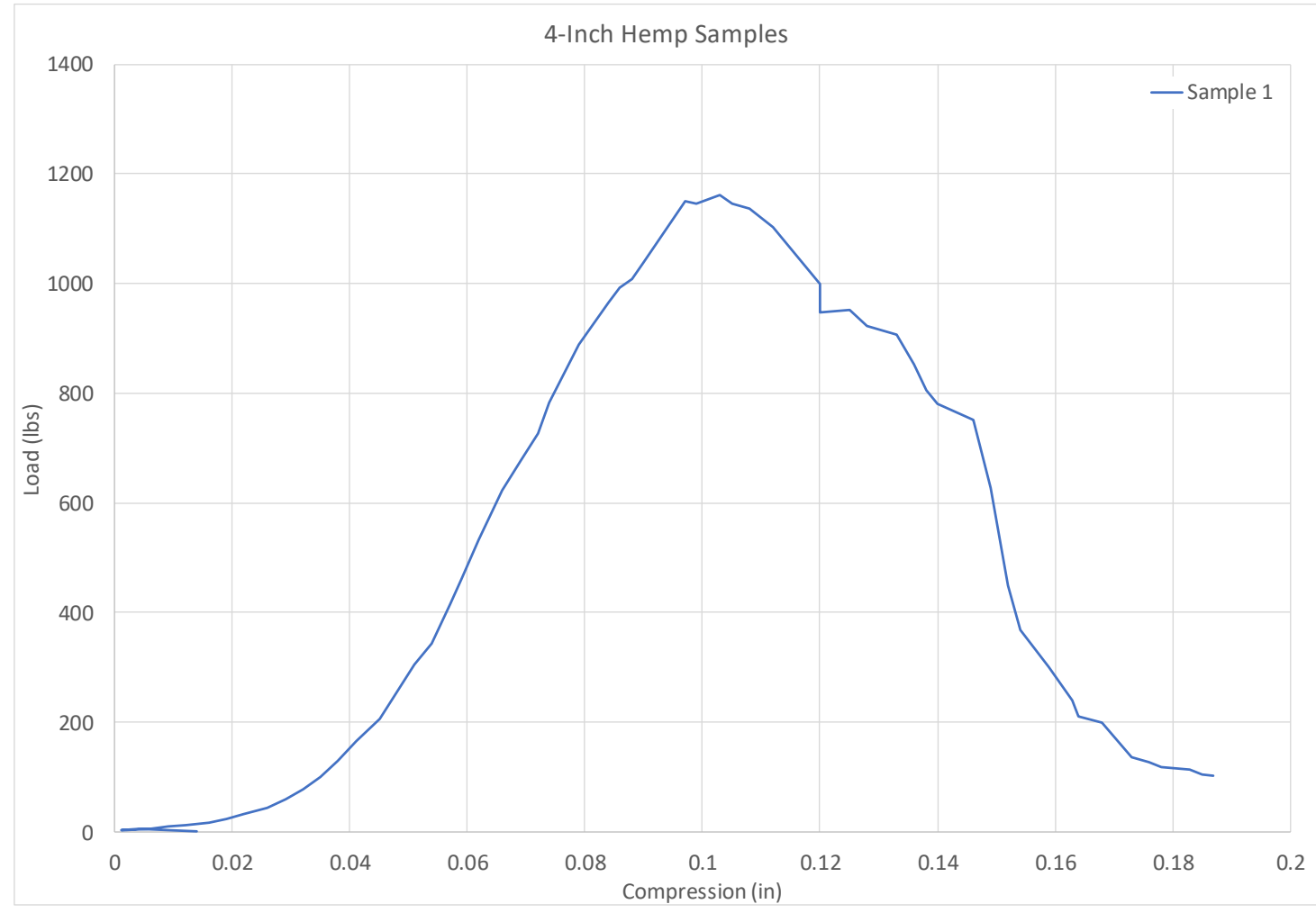

Figure 41: 4-Inch Hemp Fiber Load-Displacement Data 


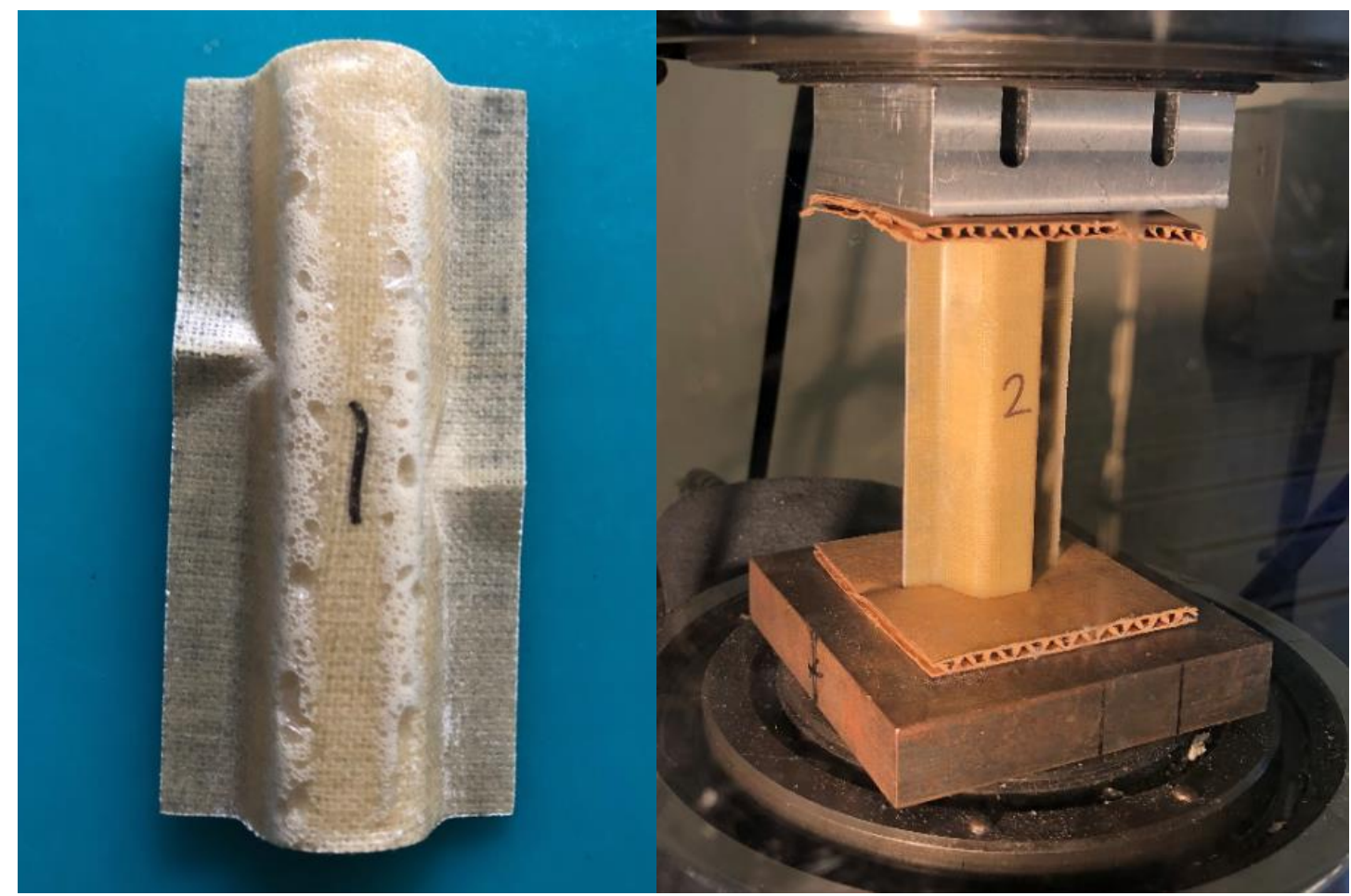

Figure 42: 4-Inch Hemp Fiber Compression Test with Whole Structure Buckling

Fig. 43 displays the stiffnesses

of the 4-inch hemp fiber samples. The average stiffness of 21802 pounds per inch was roughly the same as the hemp fiber samples at the 2- and 3inch length. This is again due to the material and susceptibility to buckling of this cross-sectional shape at longer lengths. It was again expected, however, that the stiffness

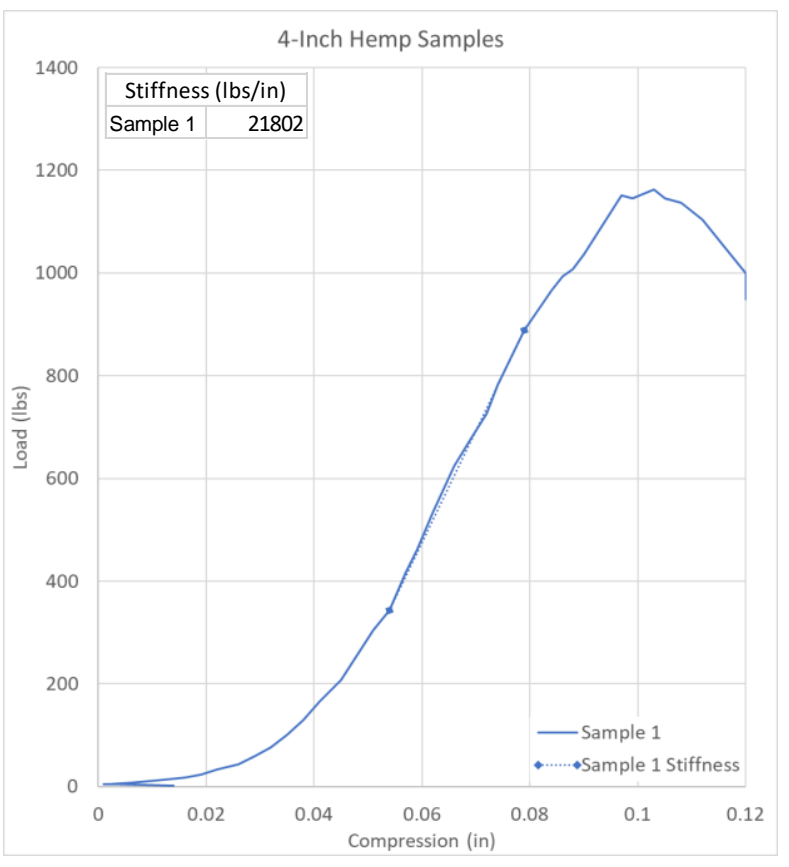

Figure 43: 4-Inch Hemp Fiber Stiffnesses would decrease even further with the increase in length. 
There was only one sample for this test due to data capture error of the other samples and a lack of identical material. This extra susceptibility to buckling causes even the slightest misalignment of the sample to cause drastic outliers, not representative of the sample properties. Even with more samples, buckling would cause unreliability.

\subsection{Carbon Core-Hemp Skin Results}

The carbon core-hemp skin samples were next to be tested. Due to differences in the materials, it was more difficult to ensure that the top and bottom surfaces were flat as each material reacted to sanding differently. It was also expected, however, that the buckling resistance of the structure would be impacted by the differences in stiffness of the two materials. Again, the goal with the mixed material samples was to remedy early debonding and leverage the properties of both materials.

\subsubsection{1-Inch Length}

The first two 1-inch carbon core-hemp skin samples yielded at a load much higher than that of the second two samples. This could be due to the second two samples being slightly shorter than the first two, although all are still very close to one inch. This can be seen in Fig. 44. This will be discussed further along with other error discussion in the results

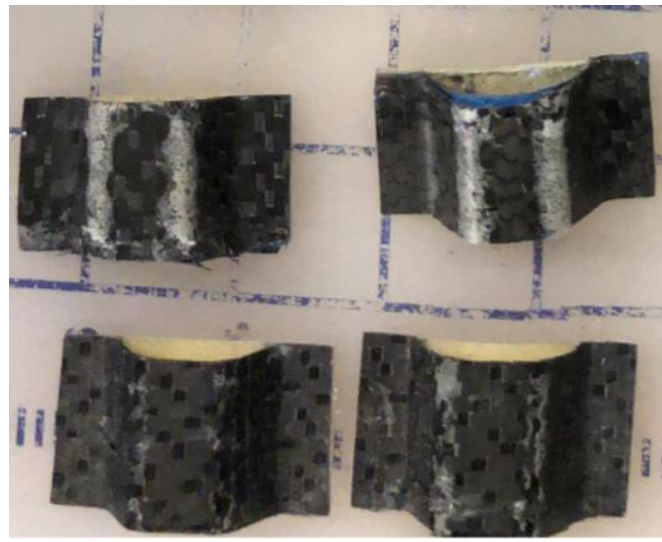

Figure 44: 1-Inch Carbon Core-Hemp Skin Samples (1 and 2 on Bottom, 3 and 4 on Top) and discussion section. 
Table 20 lists the ultimate load each sample held before failures. The average ultimate load that the samples held was 1196 pounds. Based on the cross-sectional area of the sample, Table 21 lists the ultimate stress each sample held. The average ultimate stress that the samples held was 11435 pounds per square inch. This ultimate stress was again derived from the cross-sectional area but, in this case with two different material thicknesses, the total cross-sectional area was in between that of the full hemp and full carbon fiber samples. Despite this, the stresses held by the carbon core-hemp skin samples were much higher than both the all-carbon fiber and the all-hemp fiber samples at the 1inch length.

Table 20: 1-Inch Carbon Core-Hemp Skin Ultimate Loads

\begin{tabular}{|rrrrrrr|}
\hline \multicolumn{7}{|c|}{ Ultimate Load (lbs) } \\
Sample 1 & Sample 2 & Sample 3 & Sample 4 & AVG & STDEV \\
\hline 1368 & 1351 & 990 & 1076 & 1196 & 191.727 \\
\hline
\end{tabular}

Table 21: 1-Inch Carbon Core-Hemp Skin Ultimate Stresses

\begin{tabular}{|c|rrrr|}
\hline \multicolumn{5}{|c|}{ Ultimate Stress (lbs/in^2) } \\
\hline Sample 1 & Sample 2 & Sample 3 & Sample 4 & AVG \\
\hline 11596 & 11555 & 8395 & 14195 & 11435 \\
\hline
\end{tabular}

A very prominent feature of this configuration's compression test was the blocking of buckling behavior towards the carbon fiber corrugation. This can be seen in Fig. X, as the hemp skin to the right is seen slightly bowing out to the right before snapping back to the left and buckling with the carbon fiber. 


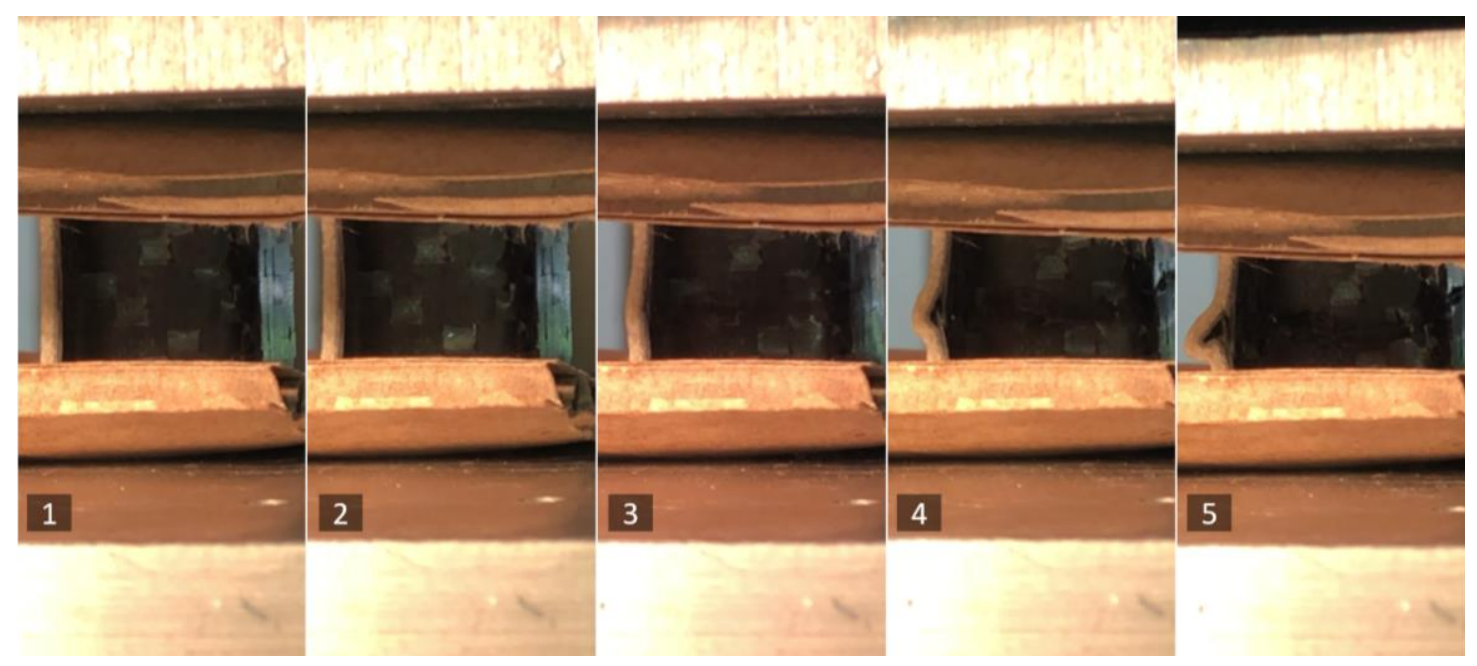

Figure 45: 1-Inch Carbon Core-Hemp Skin Samples Buckling Progression Side View

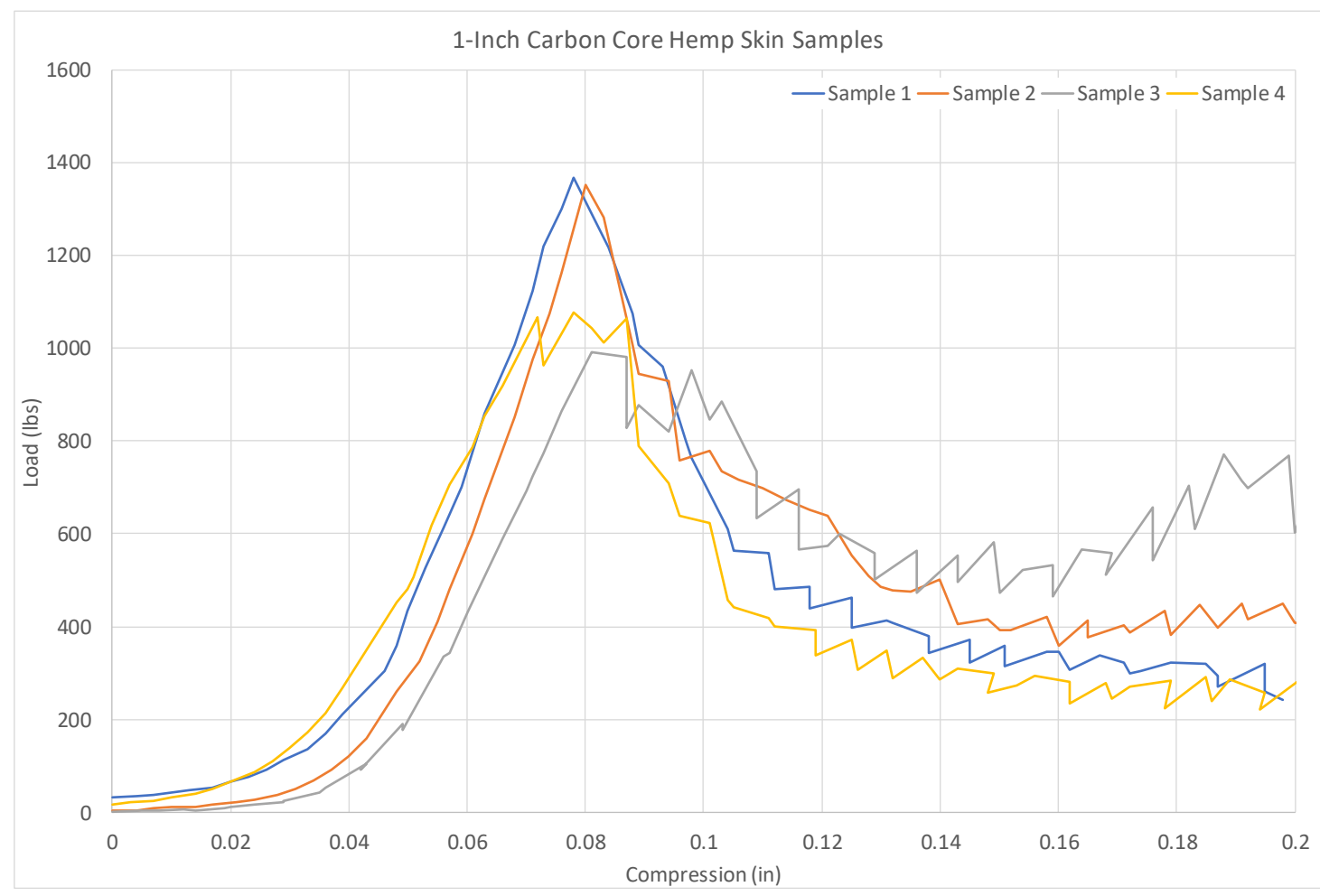

Figure 46: 1-Inch Carbon Core-Hemp Skin Load-Displacement Data

The load-displacement curves for the 1-inch carbon core-hemp skin samples, seen in Fig. 46, display similar failure modes among the first two and second two samples. The first two samples load smoothly up to the ultimate load at which they fail quite drastically. 
The second two samples fail gradually at the ultimate load. This somewhat coincides with the length of the samples as the first two samples were slightly longer than the second two samples. This, however, does not explain why the second two samples failed much more gradually than the first two samples.

The post failure behavior of these samples, especially samples 2 and 3, show that even after failure, the samples continue to hold a significant load. This load reaches near 800 pounds for sample 3 and is a feature of the all-carbon fiber samples as well. This suggests that the carbon fiber core is the cause of this beneficial behavior.

Fig. 47 displays the stiffnesses of each of the carbon core-hemp skin 1-inch samples as well as the linear section used to calculate them. The average stiffness of the four samples was 30664 pounds per inch. This was higher than that of both the all hemp fiber and all carbon fiber samples.

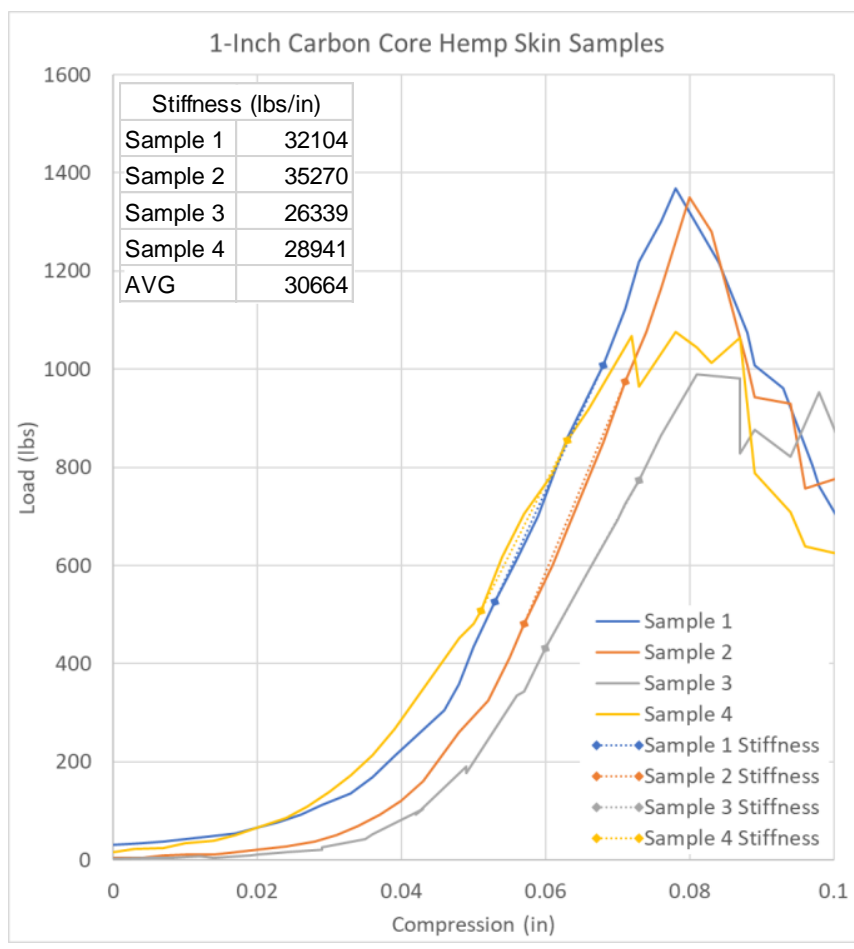

Figure 47: 1-Inch Carbon Core-Hemp Skin Stiffnesses 
This, in addition to the smooth loading curves with a single failure mode, can be attributed to the resistance of the hemp-carbon fiber interface to surface debonding. As can be seen in Fig. X, even though the left side of the structure buckles, at least the left most lamina of the carbon still stays bonded to the hemp skin.

\subsubsection{2-Inch Length}

Each of the 2-inch carbon core-hemp skin samples failed at very different load forces, with sample 3 withstanding much higher loads than the first two samples. Table 22 lists the ultimate load each sample held before failures. The average ultimate load that the samples held was 1293 pounds. Based on the cross-sectional area of the sample, Table 23 lists the ultimate stress each sample held. The average ultimate stress that the samples held was 10660 pounds per square inch. These were both higher than the 1 -inch samples but slightly lower than the 2-inch carbon fiber samples. Sample 3 failed much differently than samples 1 and 2, buckling in the center of the structure as opposed to along the top edge.

Table 22: 2-Inch Carbon Core-Hemp Skin Ultimate Loads

\begin{tabular}{|r|r|r|r|r|}
\hline \multicolumn{5}{|c|}{ Ultimate Load (lbs) } \\
\hline Sample 1 & Sample 2 & Sample 3 & AVG & STDEV \\
\hline 1270 & 1163 & 1447 & 1293 & 143.296 \\
\hline
\end{tabular}

Table 23: 2-Inch Carbon Core-Hemp Skin Ultimate Stresses

\begin{tabular}{|rrrr|}
\hline \multicolumn{4}{|c|}{ Ultimate Stress (lbs/in^2) } \\
\hline Sample 1 & Sample 2 & Sample 3 & AVG \\
\hline 10688 & 9234 & 12058 & 10660 \\
\hline
\end{tabular}




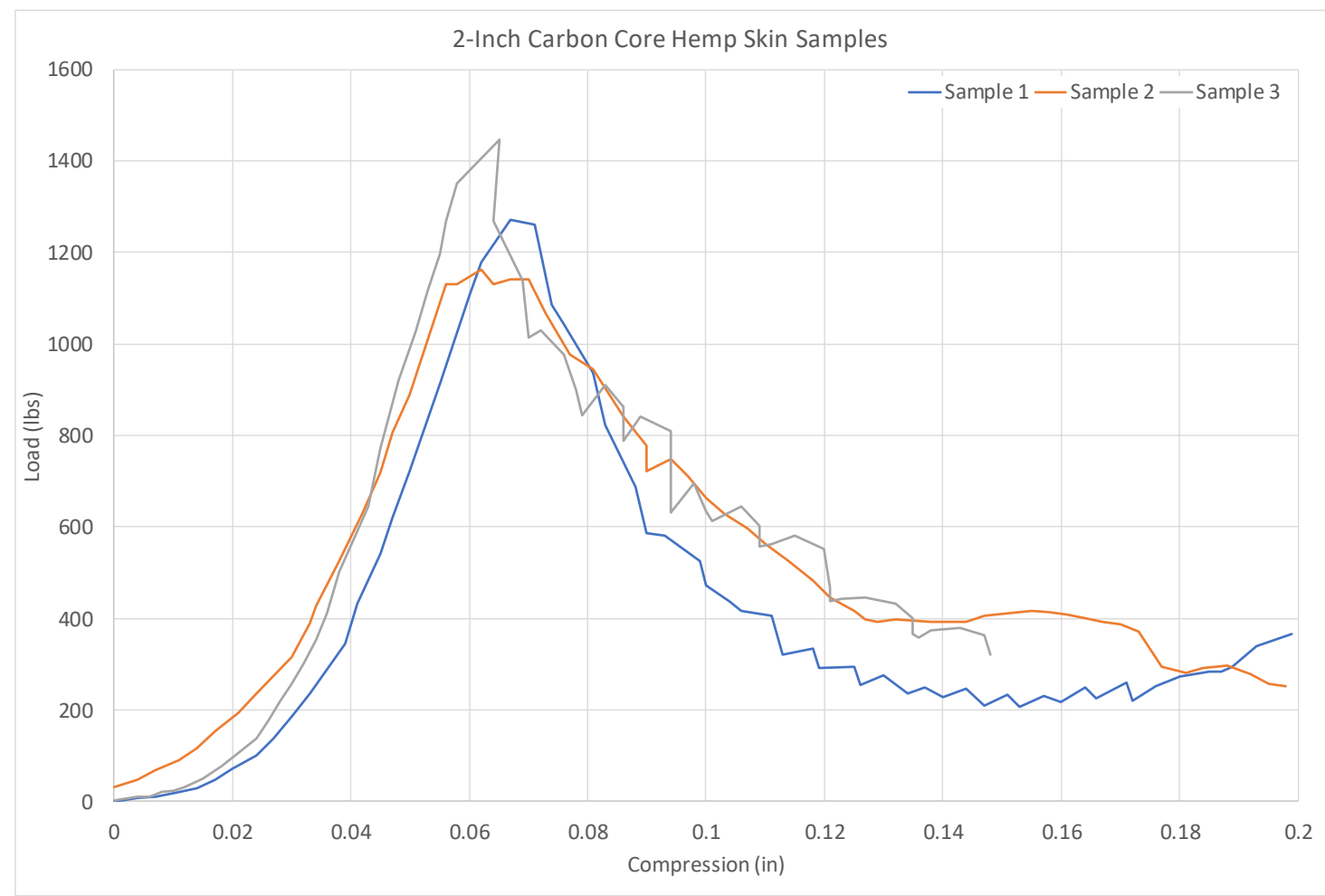

Figure 48: 2-Inch Carbon Core-Hemp Skin Load-Displacement Data

The load-displacement curves for the 2-inch carbon core-hemp skin samples, seen in Fig. 48, display similar stiffness slopes throughout the loading region of the curve. Again, the ability of the hemp to adhere to and buckle with the carbon fiber allows for curves free of multiple failures due to debonding and early buckling.

As mentioned before, the third sample somewhat differently than the first two, seen in Fig. 49 below. It buckled in the middle of the corrugation as opposed to the top of the corrugation. This results in a higher ultimate load and stress as well as a much steeper curve, indicating a much higher stiffness. This supports that the sample was much more stable axially, having less eccentric loading. The sample thus buckles later, to a lesser degree, and withstands a greater load. 


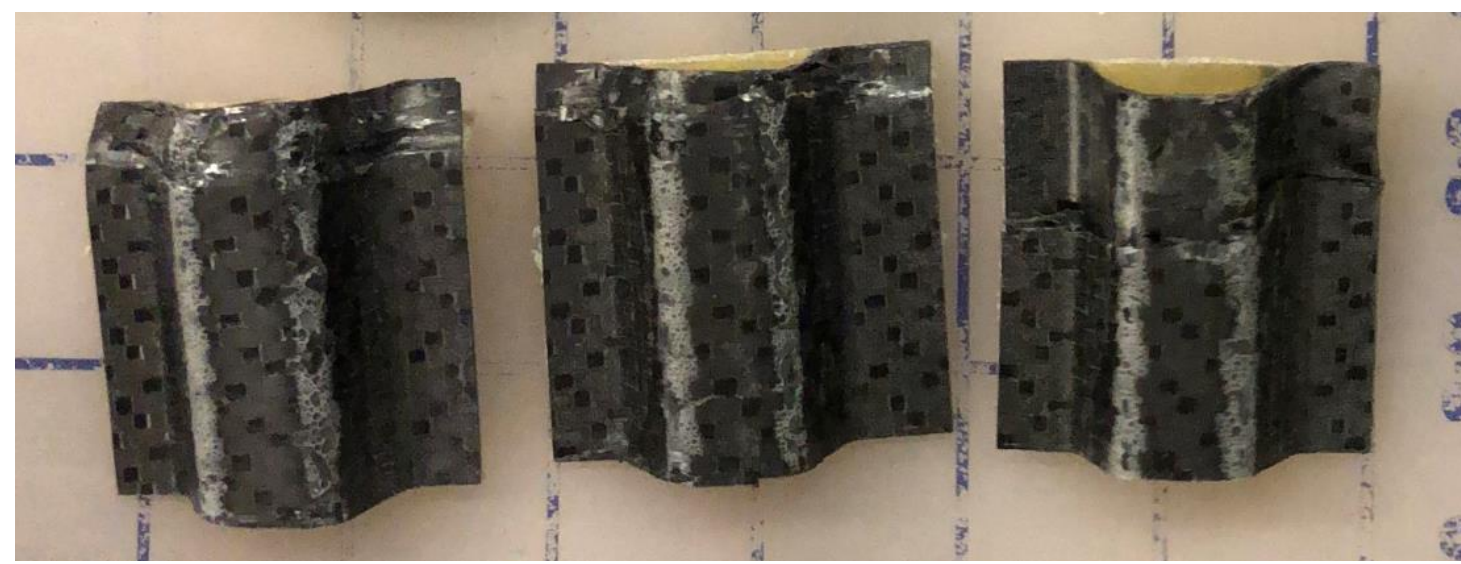

Figure 49: 2-Inch Carbon Core-Hemp Skin Compression Test Failed Samples

The average stiffness of

the 2-inch carbon core-hemp skin samples of 38528 pounds per inch was significantly greater than that of the 1 -inch samples. This increase of stiffness with the increase in length mirrored the trend with the carbon fiber samples but was opposite of the trend shown in the hemp fiber samples. This 2-

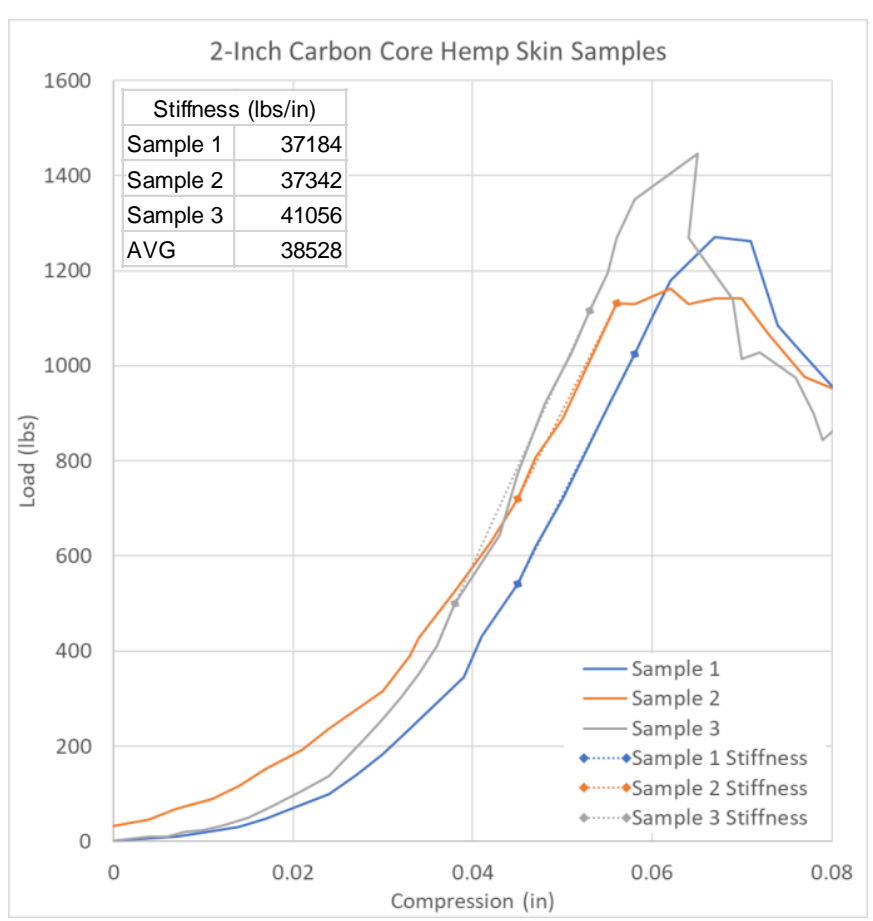

Figure 50: 2-Inch Carbon Core-Hemp Skin Stiffnesses

inch stiffness value was very close to that of the carbon fiber value of 38547 pounds per inch. Sample 3 had a much higher stiffness than 1 or 2, corresponding to the local buckling failure mode in the center, as opposed to the edge buckling mode. This steeper load curve 
leads to a much more drastic failure initially, but loads are still sustained around $50 \%$ of the maximum at two times the failure displacement. This is one major benefit of the corrugated sandwich panel structure.

\subsubsection{3-Inch Length}

The 3-inch carbon core-hemp skin samples both failed at similar loads and with similar curves, both loading and post failure. Table 24 lists the ultimate load each sample held before failures. The average ultimate load that the samples held was 1183 pounds. Based on the cross-sectional area of the sample, Table 25 lists the ultimate stress each sample held. The average ultimate stress that the samples held was 9838 pounds per square inch. These were much lower than the 1-inch and 2-inch samples.

Table 24: 3-Inch Carbon Core-Hemp Skin Ultimate Loads

\begin{tabular}{|rr|rr|}
\hline \multicolumn{4}{|c|}{ Ultimate Load (lbs) } \\
Sample 1 & Sample 2 & AVG & STDEV \\
1238 & 1127 & 1183 & 78.1169 \\
\hline
\end{tabular}

Table 25: 3-Inch Carbon Core-Hemp Skin Ultimate Stresses

\begin{tabular}{|c|c|c|}
\hline \multicolumn{3}{|c|}{ Ultimate Stress (lbs/in^2) } \\
Sample 1 & Sample 2 & AVG \\
\hline 10197 & 9478 & 9838 \\
\hline
\end{tabular}

As stated, the load-displacement curves for the 3-inch carbon core-hemp skin samples, seen in Fig. 51, display very similar stiffness slopes throughout the loading region of the curve. This similarity is supported by the very similar appearance of the samples post-failure, seen in Fig. 52. 


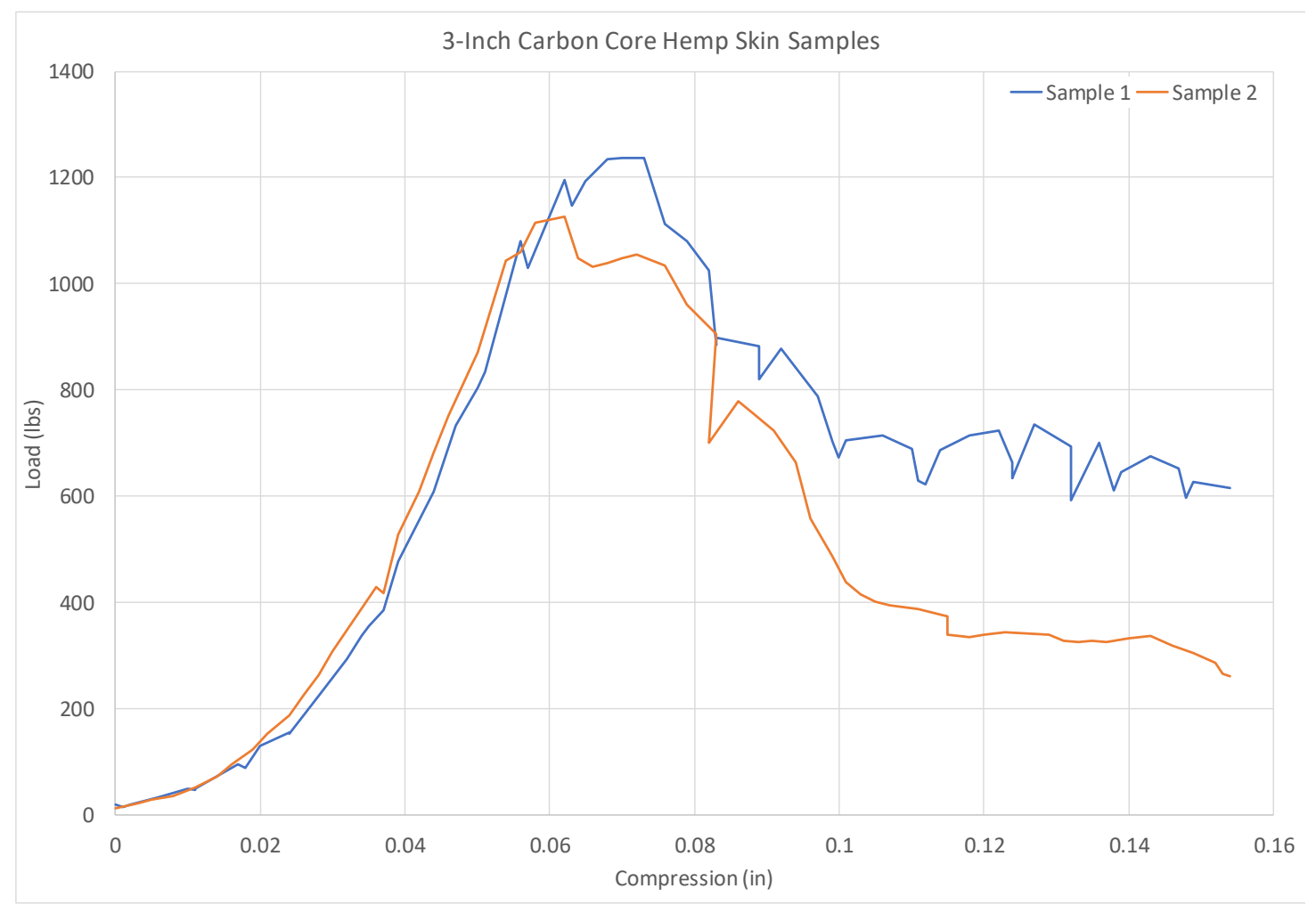

Figure 51: 3-Inch Carbon Core-Hemp Skin Load-Displacement Data

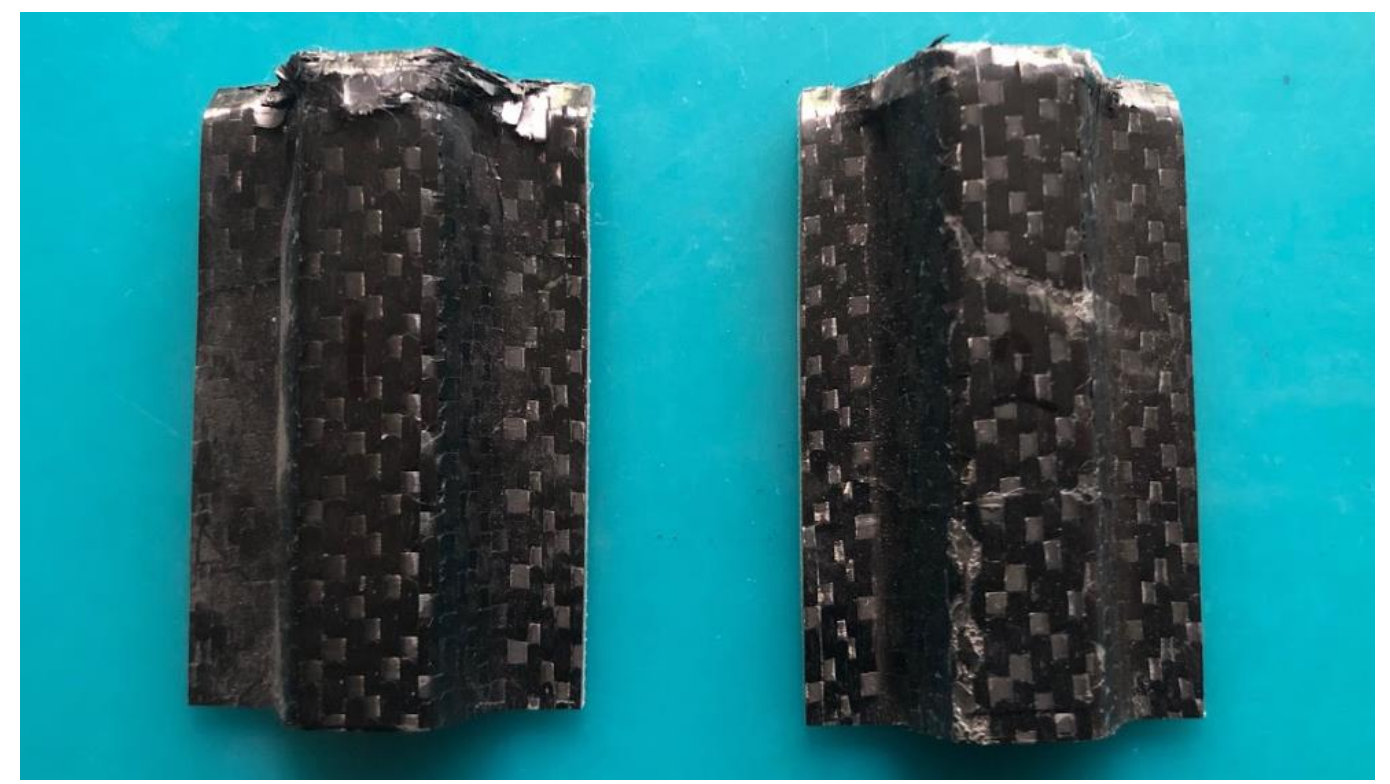

Figure 52: 3-Inch Carbon Core-Hemp Skin Compression Test Failed Samples 
The average stiffness of the 3-inch carbon core-hemp skin samples was 33482 pounds per inch, shown in Fig. 53. This was significantly less than that of both the 1-inch and 2-inch samples. Compared to these other samples, the 3-inch samples both buckled along the top edge. This shifted the top edge of the sample slightly and caused the top and bottom faces to become misaligned, resulting in eccentric loading.

As stated before, the decrease of stiffness can be attributed to the increase in length which, coupled with the edge buckling failure mode, causes more eccentric loading than the shorter samples.

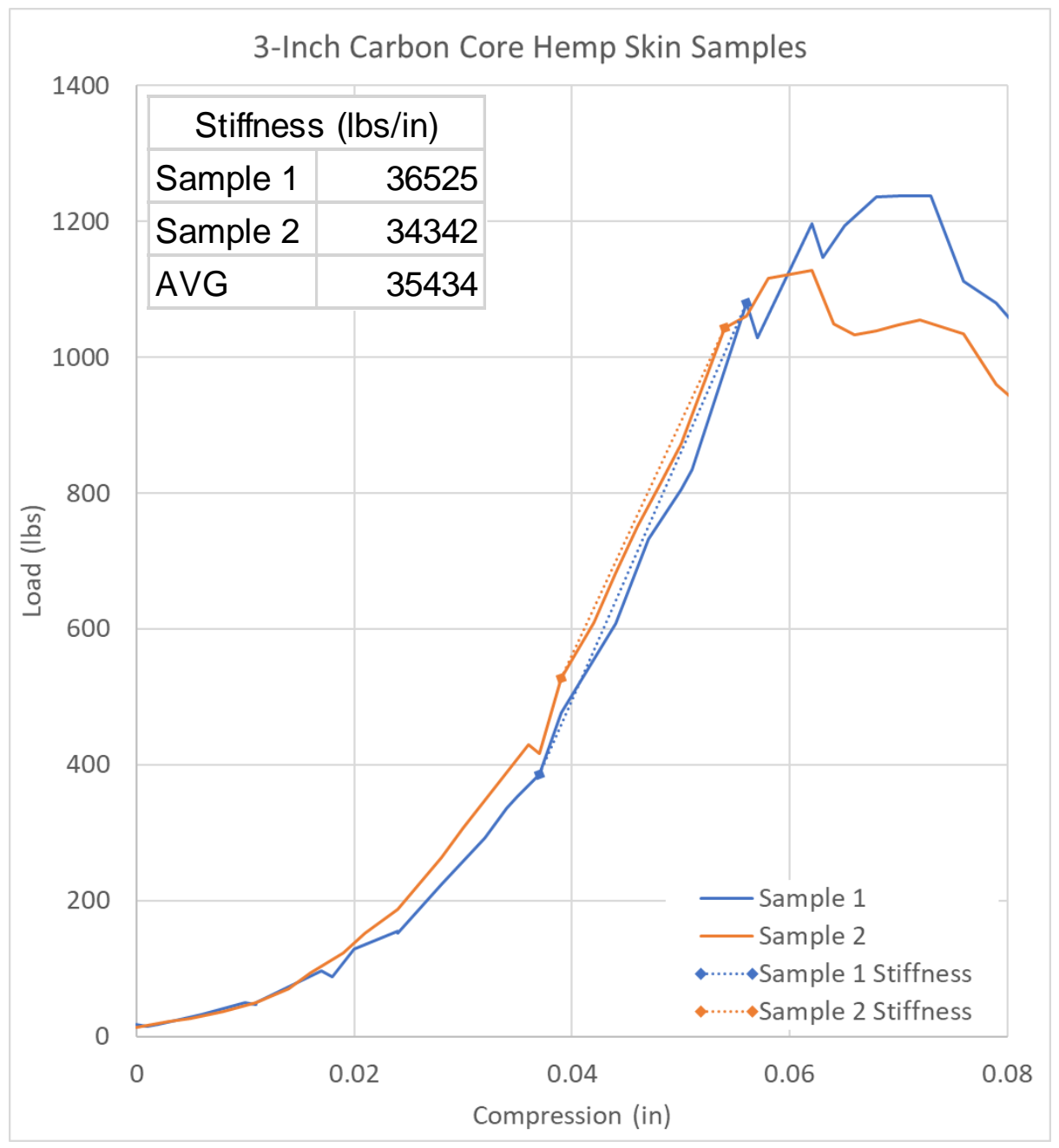

Figure 53: 3-Inch Carbon Core-Hemp Skin Stiffnesses 


\subsubsection{4-Inch Length}

Only one of the 4-inch carbon core-hemp skin samples failed in a viable way. The other was skewed early on so the test was stopped to see if the sample could be realigned. However, the damage was to great and the sample could not be used.

Table 26 lists the ultimate load the sample held before failure. The ultimate load that the viable sample held was 1390 pounds. Based on the cross-sectional area of the sample, Table 27 lists the ultimate stress each sample held. The ultimate stress that the samples held was 11538 pounds per square inch. These were much lower than the 3 -inch samples.

Table 26: 4-Inch Carbon Core-Hemp Skin Ultimate Loads Ultimate Load (lbs) Sample 1

Table 27: 4-Inch Carbon Core-Hemp Skin Ultimate Stresses Ultimate Stress (lbs/in^2) Sample 1

The load-displacement curve for the viable 4-inch carbon core-hemp skin sample was fairly smooth and had a drastic failure after ultimate load was reached, as seen in Fig. 54. This is supported by the post-failure state of the sample, seen in Fig. 55, where the buckling occurred at the middle of the carbon core. 


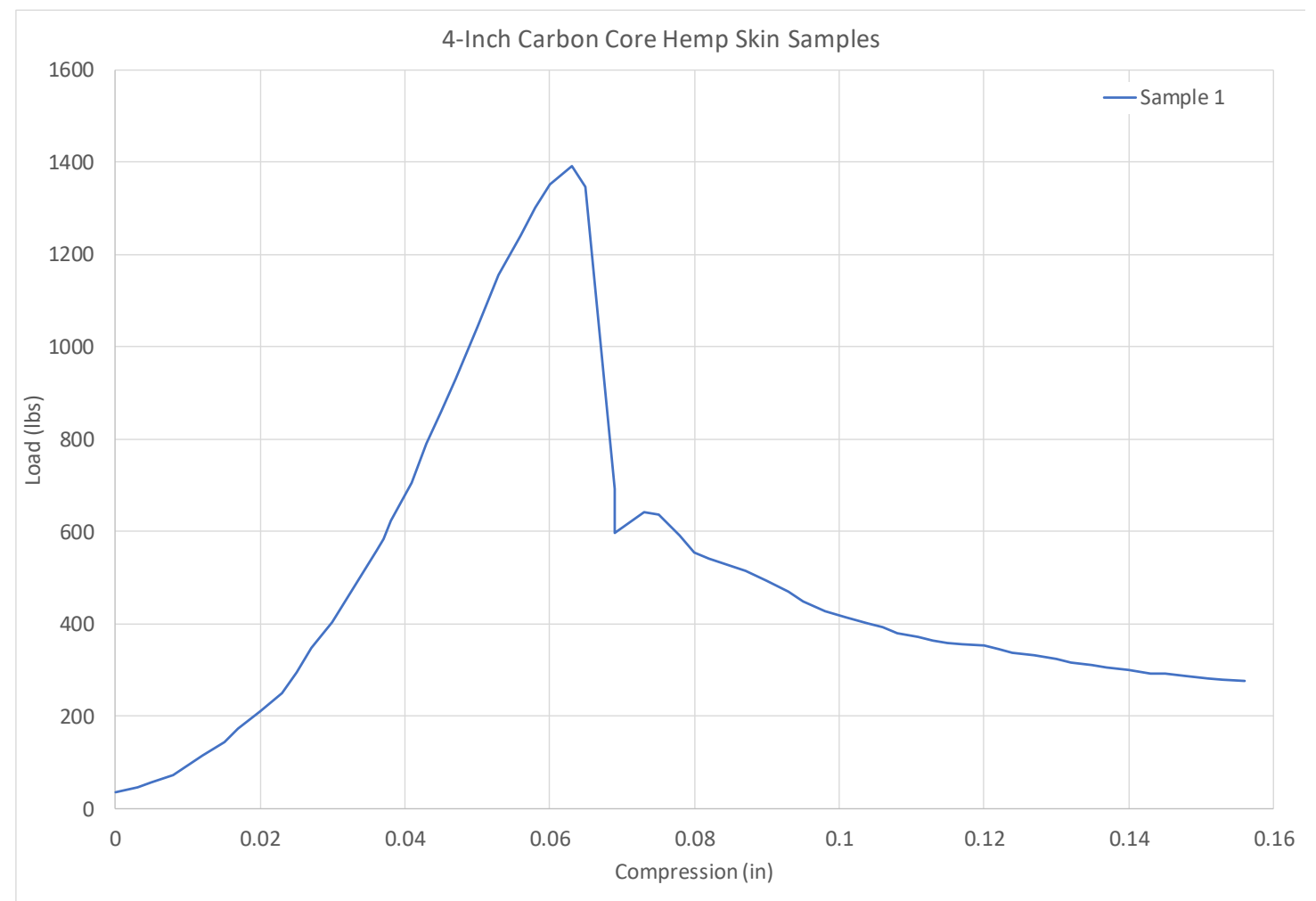

Figure 54: 4-Inch Carbon Core-Hemp Skin Load-Displacement Data

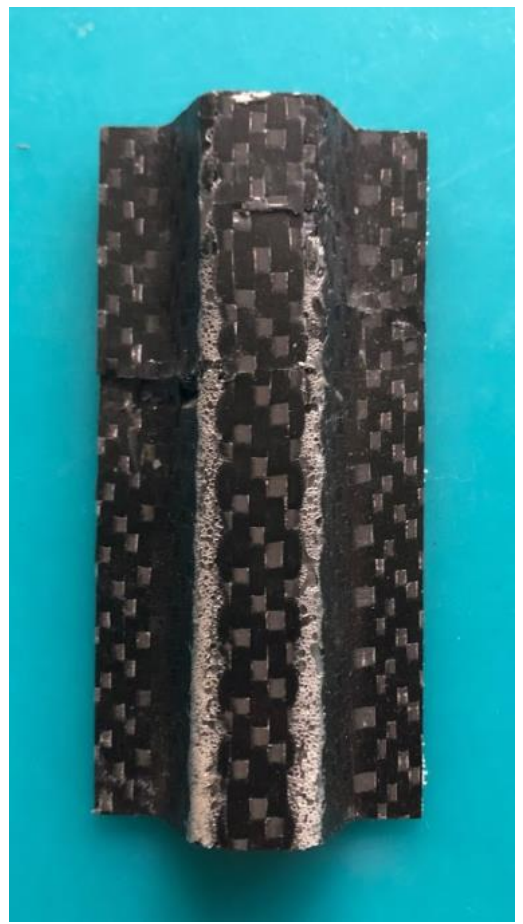

Figure 55: 4-Inch Carbon CoreHemp Skin Failed Sample

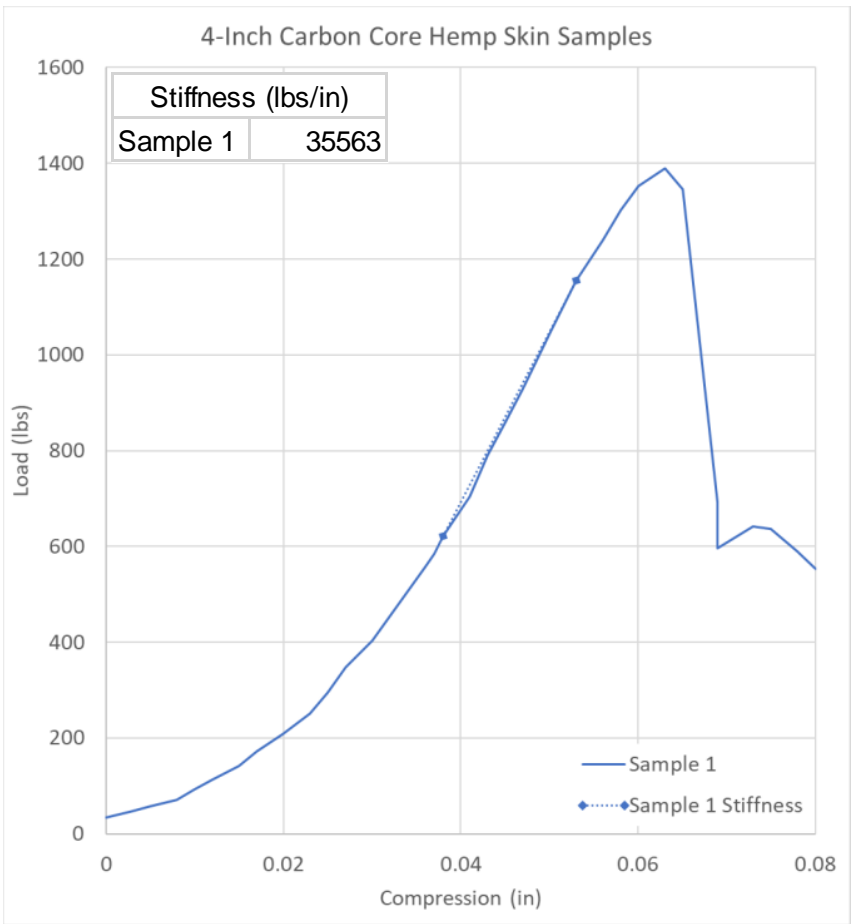

Figure 56: 4-Inch Carbon Core-Hemp Skin Stiffnesses 
The 4-inch carbon core-hemp skin sample had an average stiffness 335563 pounds per inch over the most linear section of the load-displacement curve. This was again more than that of the 3-inch samples and almost identical to that of the 4-inch carbon fiber sample. This can be seen above in Fig. 56, with the chosen linear section of the loaddisplacement curve.

Overall, the trend of the carbon core-hemp skin samples over the increasing length was very similar to the carbon fiber samples while being distinctly different from the hemp fiber samples. While these samples generally showed an increase in physical properties with an increase in length, the hemp samples generally showed a decrease in physical properties with an increase in length.

\subsection{Hemp Core-Carbon Skin Results}

The last set of samples to be tested were the hemp core-carbon skin samples. Again, it was difficult to ensure that the top and bottom surfaces were flat as each material reacted to sanding differently, but with the use of a table belt sander, the best possible surface was prepared. To help with bearing stress failure and stress concentrations that might arise from uneven surfaces, cardboard was again used as a buffer.

\subsubsection{1-Inch Length}

The 1-inch hemp core-carbon skin samples yielded at the highest load and stress of any of the 1-inch samples and the highest of any of the samples except for the 2-inch carbon fiber samples. The ultimate loads and stresses were also very consistent compared to the 
other 1-inch samples. Table 28 lists the ultimate load each sample held before failures. The average ultimate load that the samples held was 1444 pounds. Based on the cross-sectional area of the sample, Table 29 lists the ultimate stress each sample held. The average ultimate stress that the samples held was 10815 pounds per square inch. Again, this sample has a hemp corrugated section and a carbon flat plate skin, so the cross-section needed to be carefully measured to calculate the area used in the conversion from load to stress.

Table 28: 1-Inch Hemp Core-Carbon Skin Ultimate Loads

\begin{tabular}{|r|r|r|r|r|}
\hline \multicolumn{5}{|c|}{ Ultimate Load (lbs) } \\
Sample 1 & Sample 2 & Sample 3 & AVG & STDEV \\
1395 & 1472 & 1466 & 1444 & 42.5511 \\
\hline
\end{tabular}

Table 29: 1-Inch Hemp Core-Carbon Skin Ultimate Stresses

\begin{tabular}{|r|r|r|r|}
\hline \multicolumn{4}{|c|}{ Ultimate Stress (lbs/in^2) } \\
\hline Sample 1 & Sample 2 & Sample 3 & AVG \\
\hline 10579 & 11168 & 10698 & 10815 \\
\hline
\end{tabular}

This configuration's compression test resulted in the buckling of the hemp corrugated section at the center of the sample. This local buckling has been shown to allow a higher ultimate load than buckling at the edge or global buckling. This can be seen in Fig. 57, as the samples are all shown buckled towards the hemp core. Again, these failure modes will later all be discussed in detail in a dedicated section. 


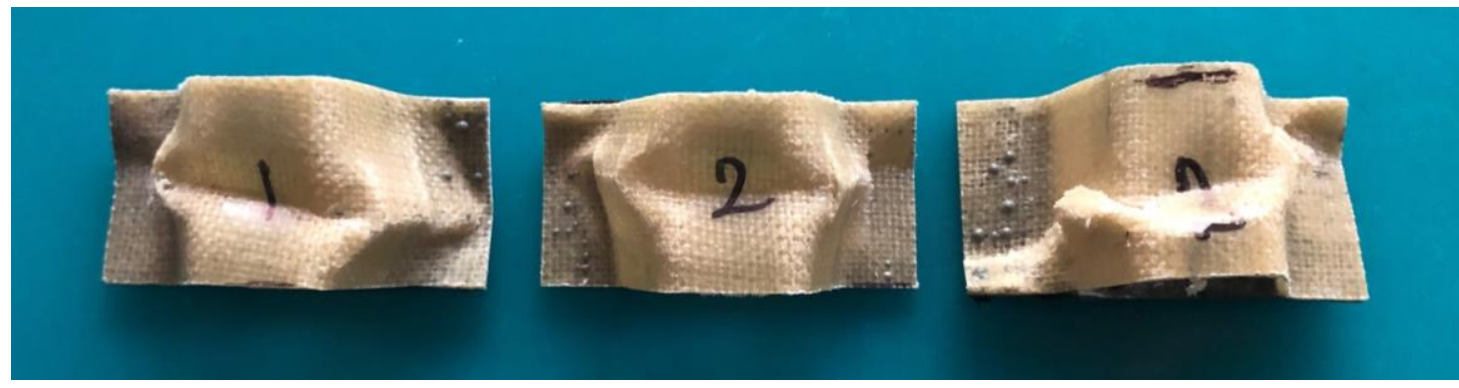

Figure 57: 1-Inch Hemp Core-Carbon Skin Compression Test Failed Samples

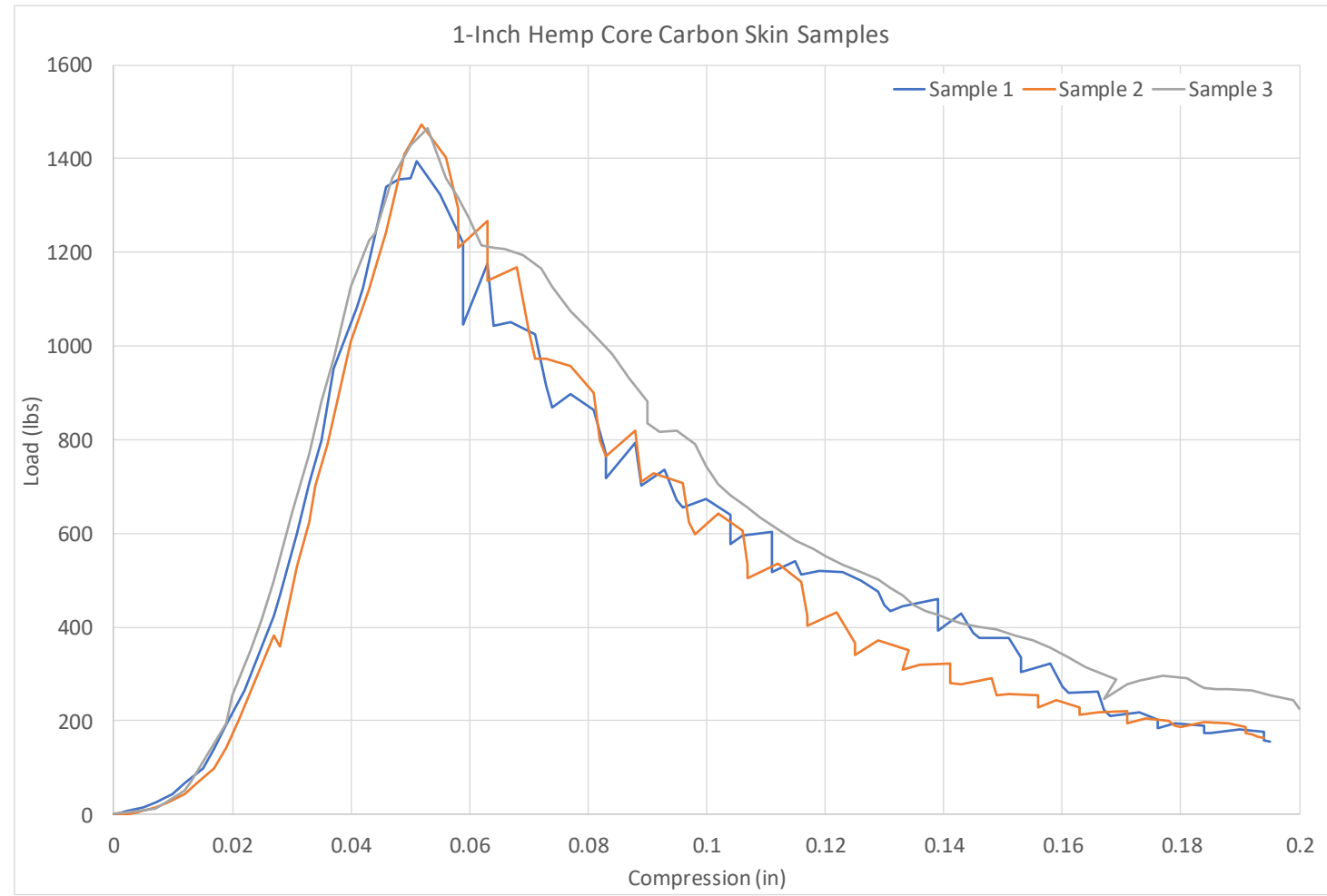

Figure 58: 1-Inch Hemp Core-Carbon Skin Load-Displacement Data

The load-displacement curves for the 1-inch hemp core-carbon skin samples, seen in Fig. 58, display almost identical failure modes among the three samples. This is marked by smooth loading up to around 1400 pounds and gradual and predictable post-failure behavior. Also seen in the curve are almost no significant dips or spikes, indicating a lack of early bonding failure. This was an issue largely prevalent in the 1-inch carbon fiber samples and diminished, but still apparent, in the 1-inch carbon core-hemp skin samples. 
These samples, like all previous samples, show strong post-failure behavior due to the corrugated structure. Even at twice the compression that ultimate load occurs, the samples all still held between 600 and 800 pounds - not an insignificant amount. This is beneficial in comparison to a structure that fails and immediately can only sustain a load that is an order of magnitude smaller than the ultimate load.

Shown in Fig. 59, the average stiffness of the 1-inch hemp core-carbon skin samples was 49510 pounds per inch. This was by far the highest of all previous samples. Again, the smooth loading curves of all samples with a single failure mode can be attributed to the resistance of the hemp-carbon fiber interface to surface debonding. The hemp core seems

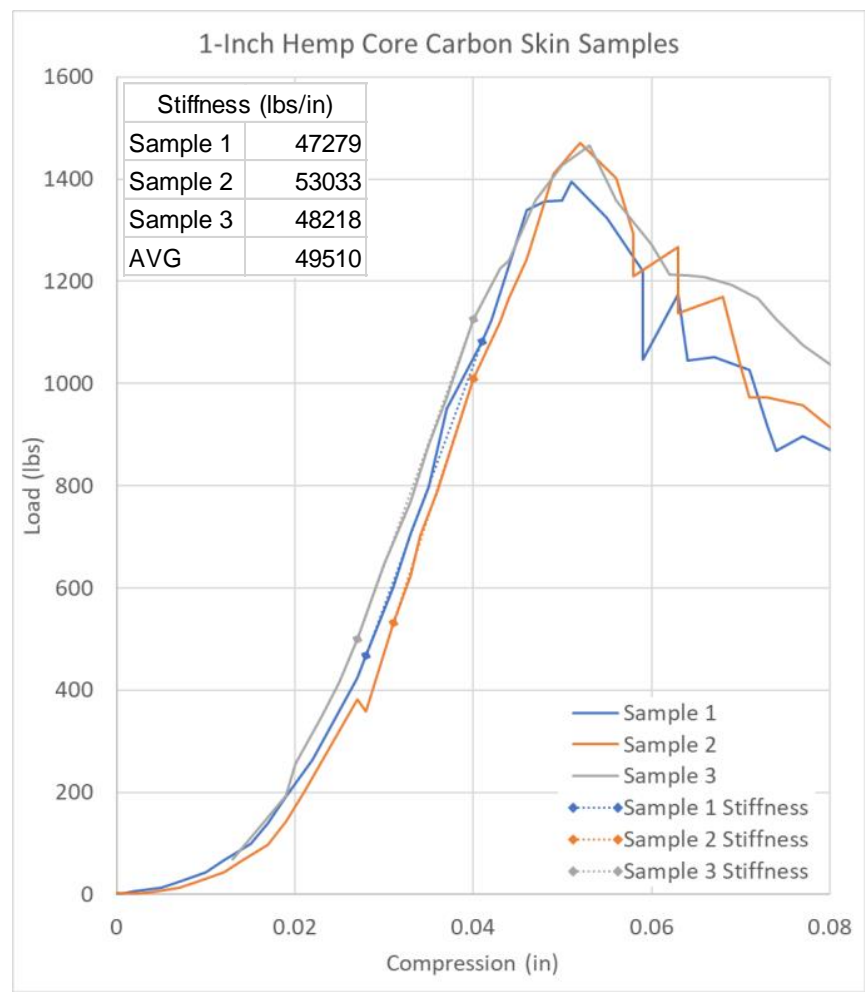

Figure 59: 1-Inch Hemp Core-Carbon Skin Stiffnesses

to adhere to the carbon skin and buckle without debonding, allowing the sample to resist much higher loads. The high performance of the 1-inch hemp core-carbon skin is strong supporting evidence that it is beneficial to have the material properties of the hemp when lower bonding surface areas are being used, as opposed to the fully carbon fiber structures tested previously. 


\subsubsection{2-Inch Length}

The ultimate load and stress of the 2-inch hemp core-carbon skin samples fell a great amount from the 1-inch samples. Compared to the other 2-inch samples, the ultimate loads and stresses of the hemp core-carbon skin samples were also low, though not quite as low as the full hemp fiber samples. Table 30 lists the ultimate load each sample held before failures. The average ultimate load that the samples held was 1274 pounds. Based on the cross-sectional area of the sample, Table 31 lists the ultimate stress each sample held. The average ultimate stress that the samples held was 9474 pounds per square inch.

Table 30: 2-Inch Hemp Core-Carbon Skin Ultimate Loads

\begin{tabular}{|c|c|c|c|}
\hline \multicolumn{4}{|c|}{ Ultimate Load (lbs) } \\
Sample 1 & Sample 2 & AVG & STDEV \\
\hline 1224 & 1324 & 1274 & 70.3486 \\
\hline
\end{tabular}

Table 31: 2-Inch Hemp Core-Carbon Skin Ultimate Stresses

\begin{tabular}{|r|r|r|}
\hline \multicolumn{3}{|c|}{ Ultimate Stress (lbs/in^2) } \\
\hline Sample 1 & Sample 2 & AVG \\
\hline 8979 & 9968 & 9474 \\
\hline
\end{tabular}

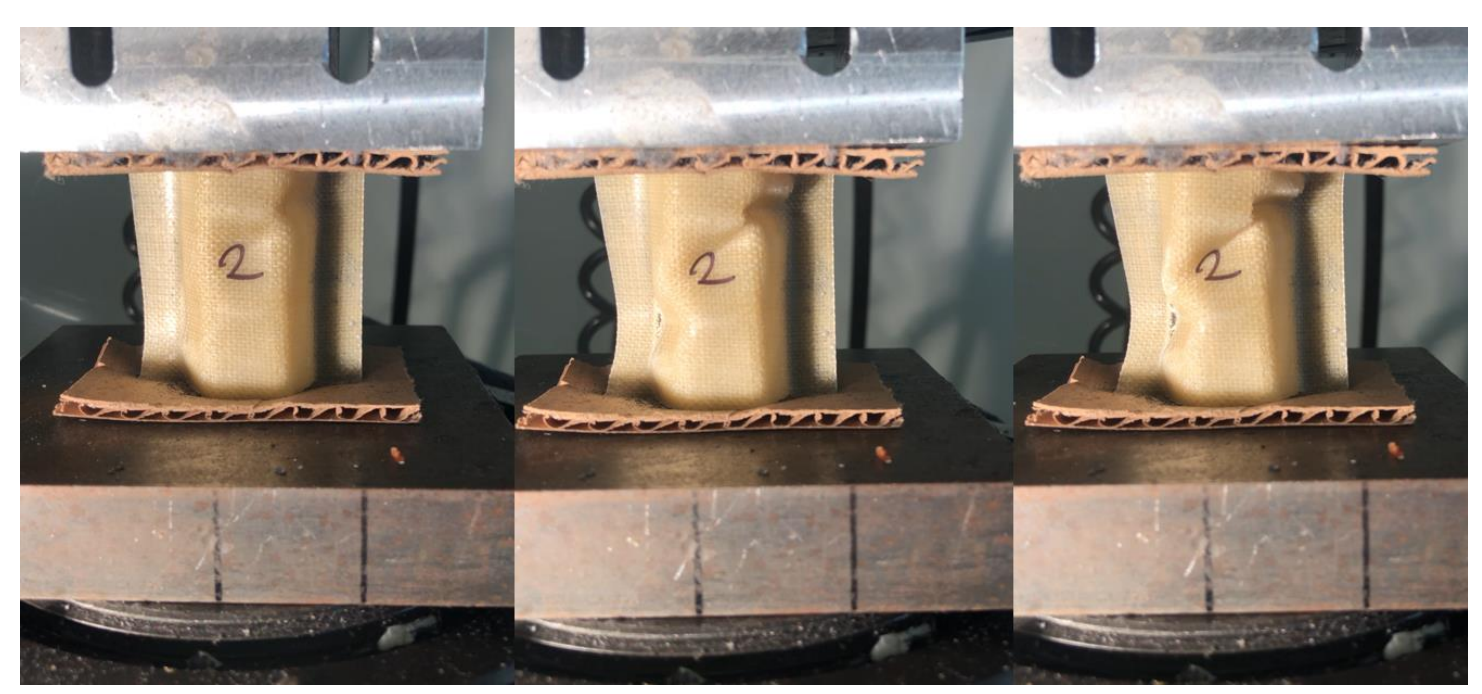

Figure 60: 2-Inch Hemp Core-Carbon Skin Compression Test Failed Samples 
There was a significant difference between the samples in terms of their mechanical properties which corresponded with the way they failed. Seen in Fig. 60, sample 2 of the hemp core-carbon skin buckled laterally, indicating unevenness along the cut. This is different than the buckling of the samples towards the top as seen previously.

Seen in Fig. 61, the load-displacement curves for the 2-inch hemp core-carbon skin samples display very similar curves to each other and to the 1-inch samples. The loading curve is smooth, and the failure does not exhibit a sudden drop in strength. This demonstrates a crumpling or yielding failure behavior as opposed to a fracture as seen with the carbon fiber samples.

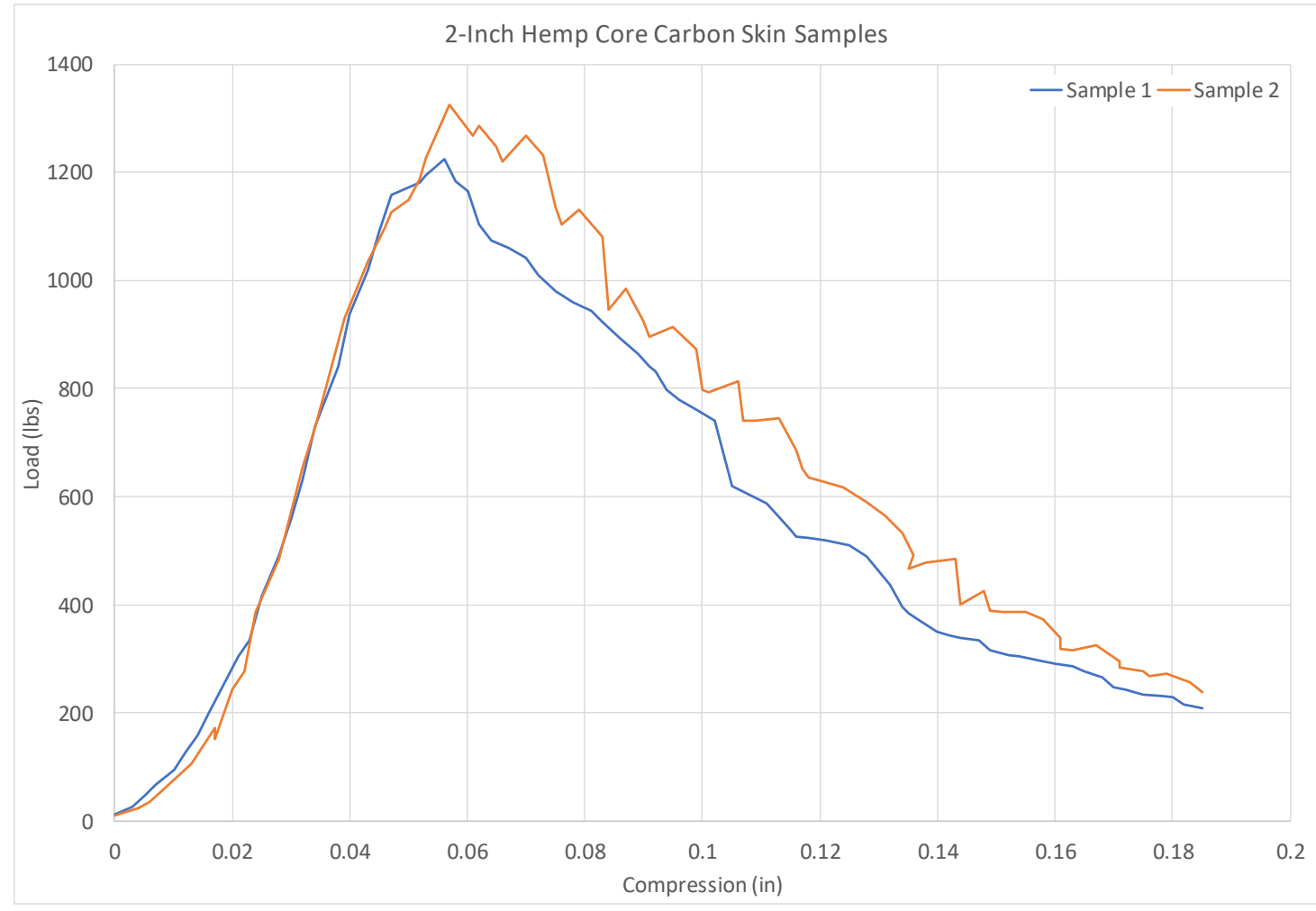

Figure 61: 2-Inch Hemp Core-Carbon Skin Load-Displacement Data 
As mentioned previously, the 2-inch samples did fail in different ways. The first sample buckled evenly toward the hemp corrugation in the upper half of the sample and yielded earlier while the second sample buckled unevenly in the middle but held a higher load. These can be seen in Fig. 62.

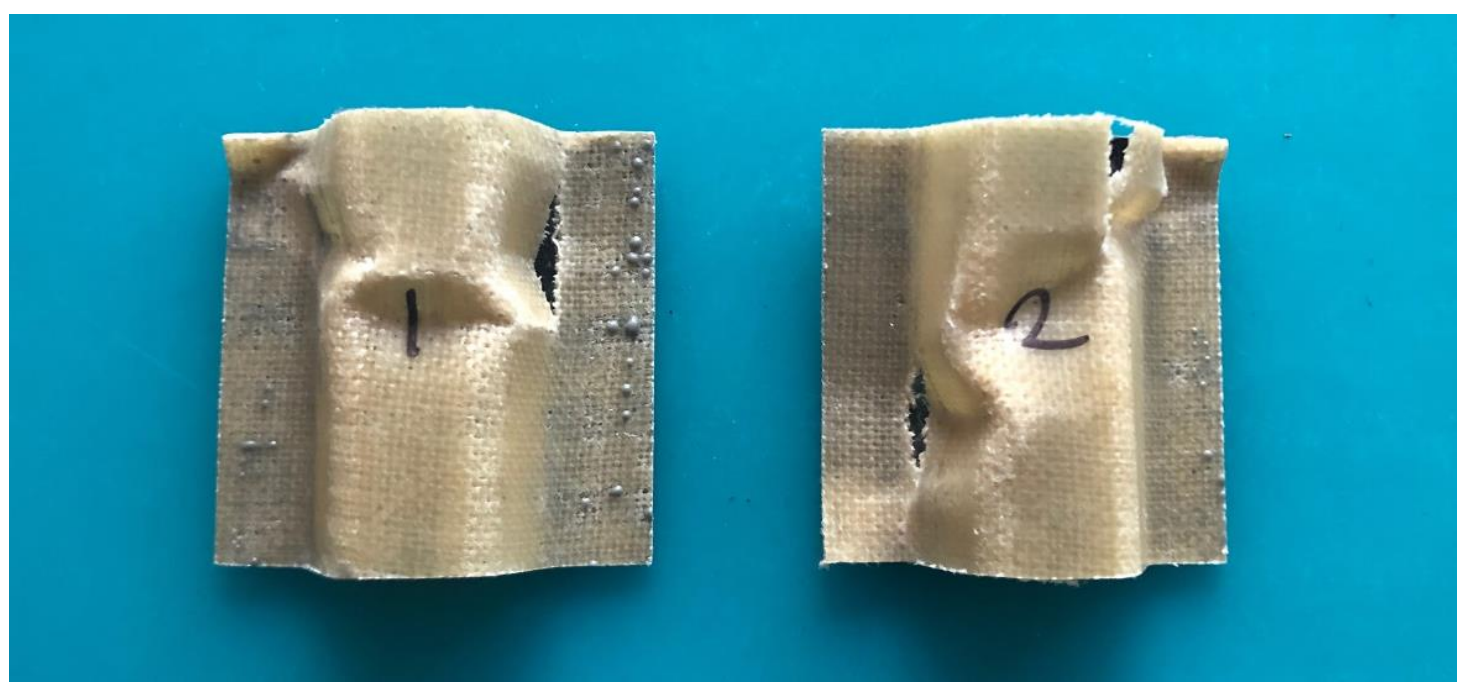

Figure 62: 2-Inch Hemp Core-Carbon Skin Compression Test Failed Samples

While there were
differences in ultimate load and
buckling behavior, these weren't
reflected as drastically in the slopes
of the linear portions of the load
curves - the stiffnesses. The
averages of the two samples were
close, within 400 pounds per inch
of each other, as seen in Fig. 63 .

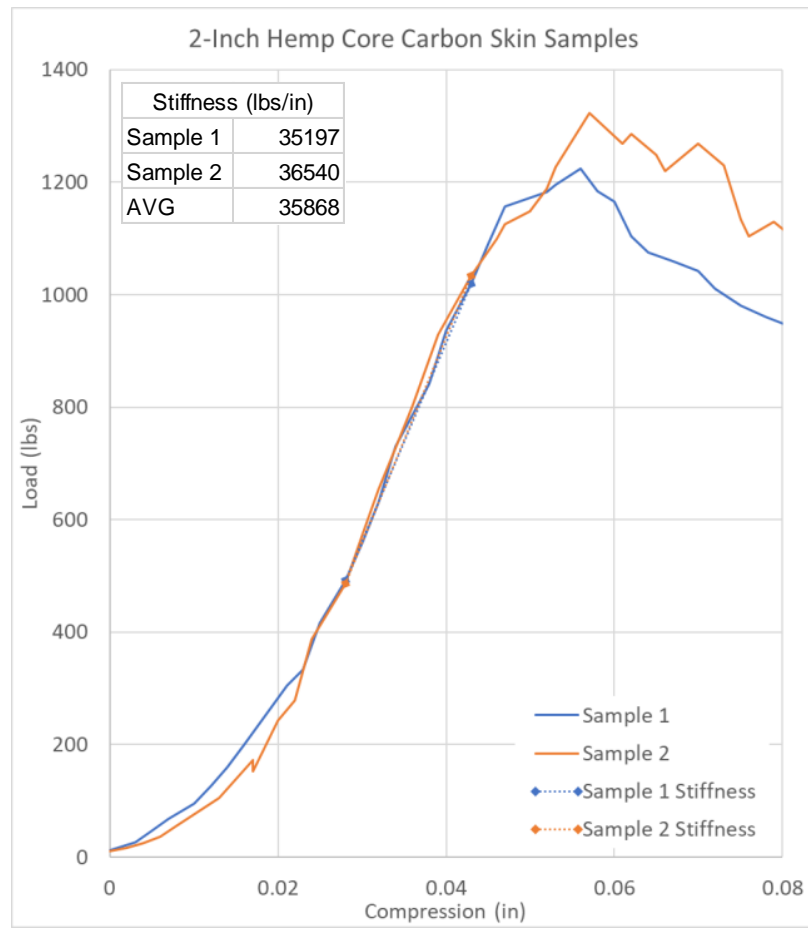

Figure 63: 2-Inch Hemp Core-Carbon Skin Stiffnesses 
The average stiffness of the 2-inch hemp core-carbon skin samples was 40676 pounds per inch. This was a significant drop from the 1 -inch samples. This stiffness was very close to all the other 2 -inch samples besides the 2-inch hemp fiber sample, which expectedly had a much lower stiffness. The 2-inch hemp core-carbon skin samples had similar smooth loading curves to their 1-inch counterparts.

\subsubsection{3-Inch Length}

The 3-inch hemp core-carbon skin samples had some of the largest deviations of load and stress of any of the samples. The average load and stress were also nearly identical to those of the 3-inch carbon core-hemp skin samples. Table 32 lists the ultimate load each sample held before failures. The average ultimate load that the samples held was 1183 pounds. Based on the cross-sectional area of the sample, Table 33 lists the ultimate stress each sample held. The average ultimate stress that the samples held was 8391 pounds per square inch. These both reflected yet another significant drop for the hemp core-carbon skin samples, although the load and stiffness of sample 1 seem like somewhat of an outlier.

Table 32: 3-Inch Hemp Core-Carbon Skin Ultimate Loads

\begin{tabular}{|r|r|r|r|r|}
\hline \multicolumn{5}{|c|}{ Ultimate Load (lbs) } \\
\hline Sample 1 & Sample 2 & Sample 3 & AVG & STDEV \\
\hline 1029 & 1185 & 1335 & 1183 & 152.905 \\
\hline
\end{tabular}

Table 33: 3-Inch Hemp Core-Carbon Skin Ultimate Stresses

\begin{tabular}{|c|c|c|c|}
\hline \multicolumn{4}{|c|}{ Ultimate Stress (lbs/in^2) } \\
\hline Sample 1 & Sample 2 & Sample 3 & AVG \\
\hline 7796 & 8917 & 10081 & 8931 \\
\hline
\end{tabular}




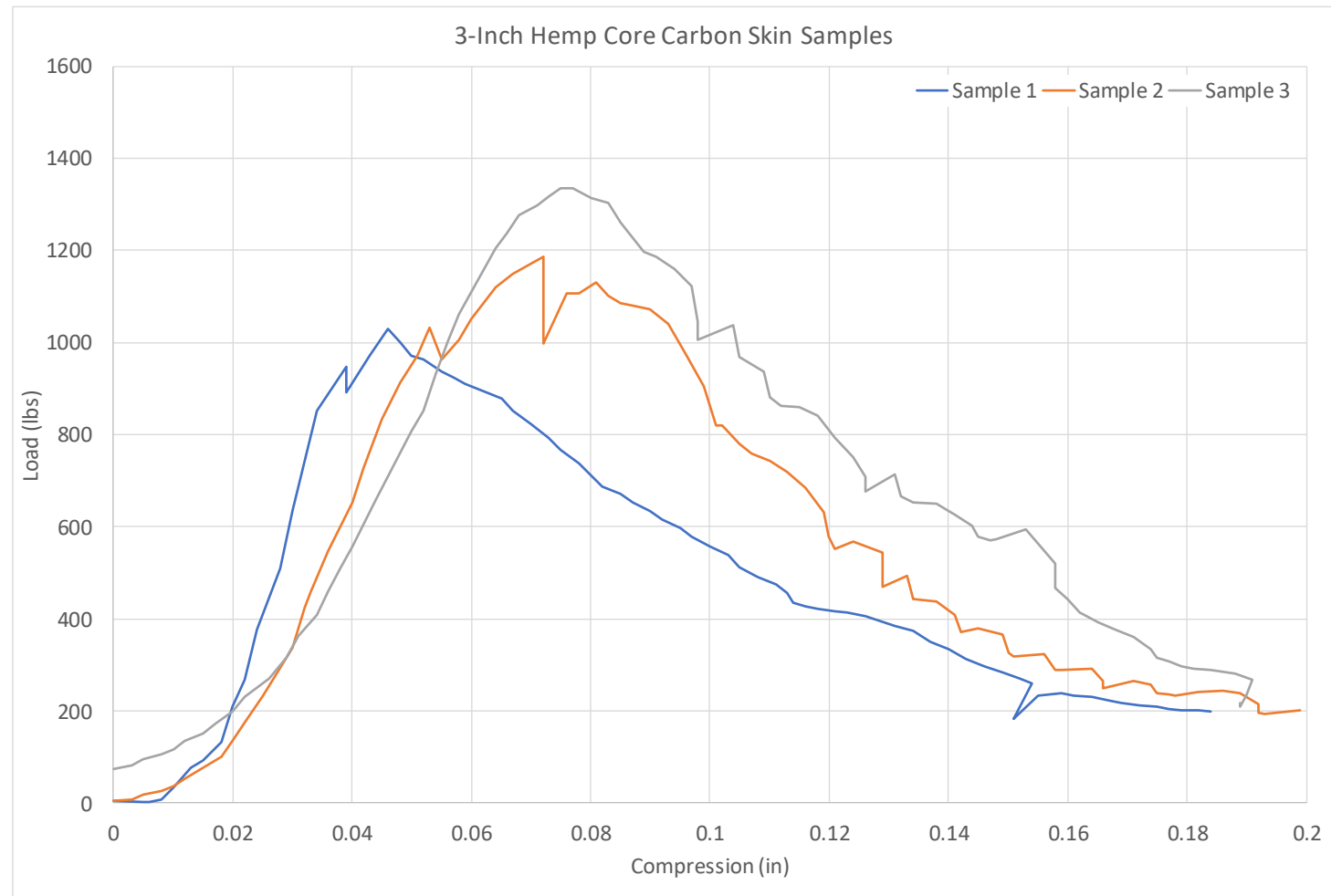

Figure 64: 3-Inch Hemp Core-Carbon Skin Load-Displacement Data

The load-displacement curves for the 3-inch hemp core-carbon skin samples, seen in Fig. 64, display almost very different failure modes between all three samples. Each sample reached a different ultimate load, had a different slope in loading, and had different post-failure behavior. Samples 1 and 2 also had small peaks before reaching ultimate load. The smoothest loading curve and most distinct failure occurred with Sample 3. This expectedly led to the highest ultimate load among the three samples of 1335 pounds.

This can be attributed to the different locations of buckling in each of the three samples, seen in Fig. 65. Sample 1 buckled at the very top, indicating eccentric loading possibly a result of unevenness between the skin and core. Sample 2 buckled towards the bottom, but not quite at the very bottom. It can also be noted that this sample had noticeable debonding between the skin and the core. Sample 3 buckled closet to the center and had the least amount of apparent debonding. These three behaviors, as stated previously, 
corresponded with the lowest to highest ultimate loads. This was opposite of the stiffnesses of each sample, which was unexpected.

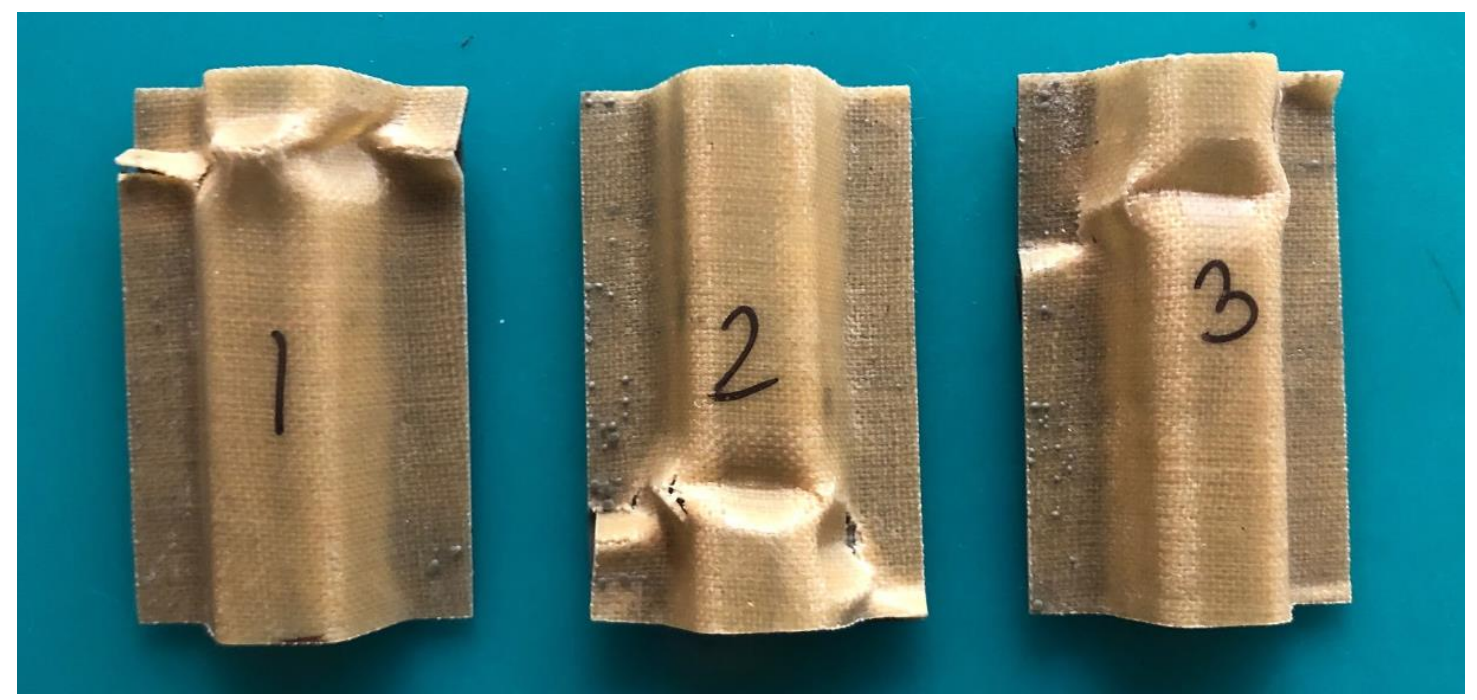

Figure 65: 3-Inch Hemp Core-Carbon Skin Compression Test Failed Samples

The stiffnesses of the three samples were all very different, with Sample 1 having a much higher stiffness than Samples 2 and 3. This is apparent in the slopes of their loading curves.

The average stiffness of the 3-inch hemp core-carbon skin samples was 39481 pounds per inch. This was lower than both the 1-inch and 2-inch samples. This

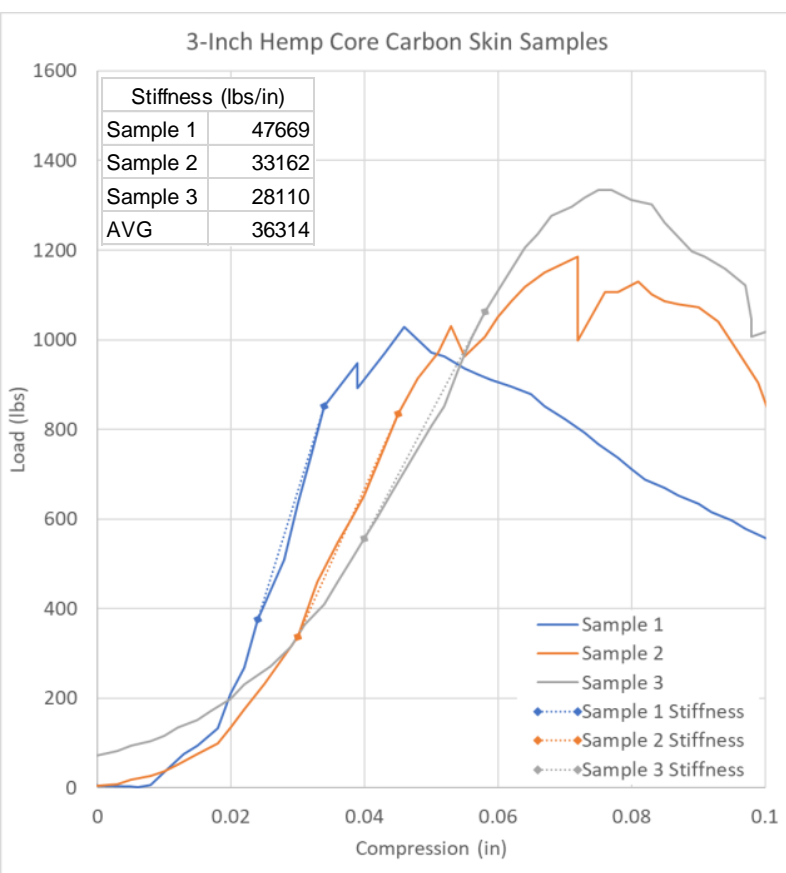

Figure 66: 3-Inch Hemp Core-Carbon Skin Stiffnesses

continued the trend of high stiffness 
for the hemp core-carbon skin samples despite the large amount of variability with this configuration and length of sample. This stiffness was second highest of the 3-inch samples to the carbon fiber samples.

The error associated with the deviation in load and stiffness could possibly be attributed to the dimensions of the samples, as the
Table 34: 3-Inch Hemp Core-Carbon Skin Sample Properties

\begin{tabular}{|l|r|r|r|r|}
\hline Part & Length & \multicolumn{1}{l|}{ Width } & Height & \multicolumn{1}{l|}{ Weight } \\
\hline $3-1$ & 2.948 & 1.805 & 0.557 & 8.7 \\
\hline $3-2$ & 2.96 & 1.821 & 0.557 & 8.6 \\
\hline $3-3$ & 2.97 & 1.812 & 0.557 & 8.3 \\
\hline
\end{tabular}

weight of each sample somewhat follows the trend of stiffness, shown in Table 34. However, most other dimensions are very similar.

\subsubsection{4-Inch Length}

The ultimate loads and stresses of the 4-inch hemp core-carbon skin samples continued the decreasing trend this sample configuration. Table 35 lists the ultimate load each sample held before failures. The average ultimate load that the samples held was 1124 pounds. Based on the cross-sectional area of the sample, Table 36 lists the ultimate stress each sample held. The average ultimate stress that the samples held was 8464 pounds per square inch. These were the lowest numbers of the 4-inch samples. One thing to note, however, was the buckling behavior of the samples during the test. This can be seen in Fig. 67 where Sample 2 is completely buckled in the middle. This was due to the length and unstable combination of the shape and mechanical properties of the sample.

Table 35: 4-Inch Hemp Core-Carbon Skin Ultimate Loads

\begin{tabular}{|r|r|r|r|}
\hline \multicolumn{4}{|c|}{ Ultimate Load (lbs) } \\
Sample 1 & Sample 2 & AVG & STDEV \\
1047 & 1202 & 1124 & 109.622 \\
\hline
\end{tabular}


Table 36: 4-Inch Hemp Core-Carbon Skin Ultimate Stresses

\begin{tabular}{|crr|}
\hline \multicolumn{3}{|c|}{ Ultimate Stress (Ibs/in^2) } \\
Sample 1 & Sample 2 & AVG \\
\hline 7965 & 8963 & 8464 \\
\hline
\end{tabular}

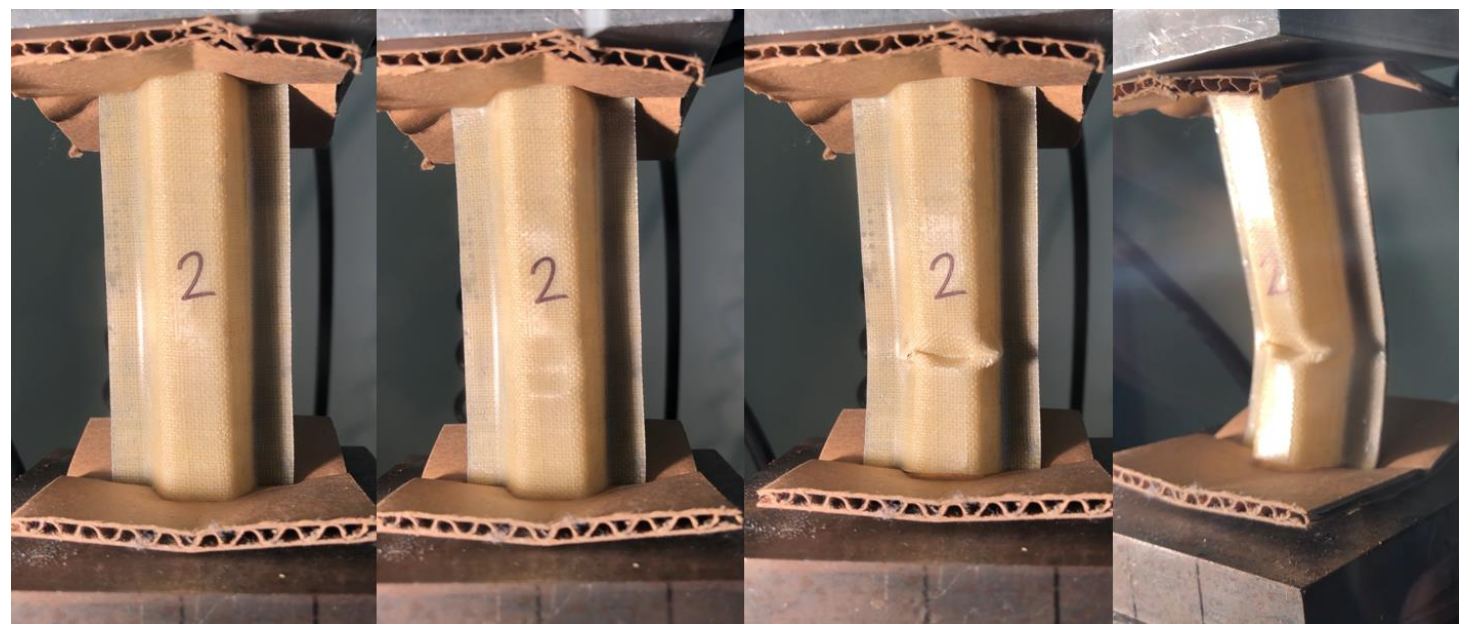

Figure 67: 4-Inch Hemp Core-Carbon Skin Compression Test Failed Samples

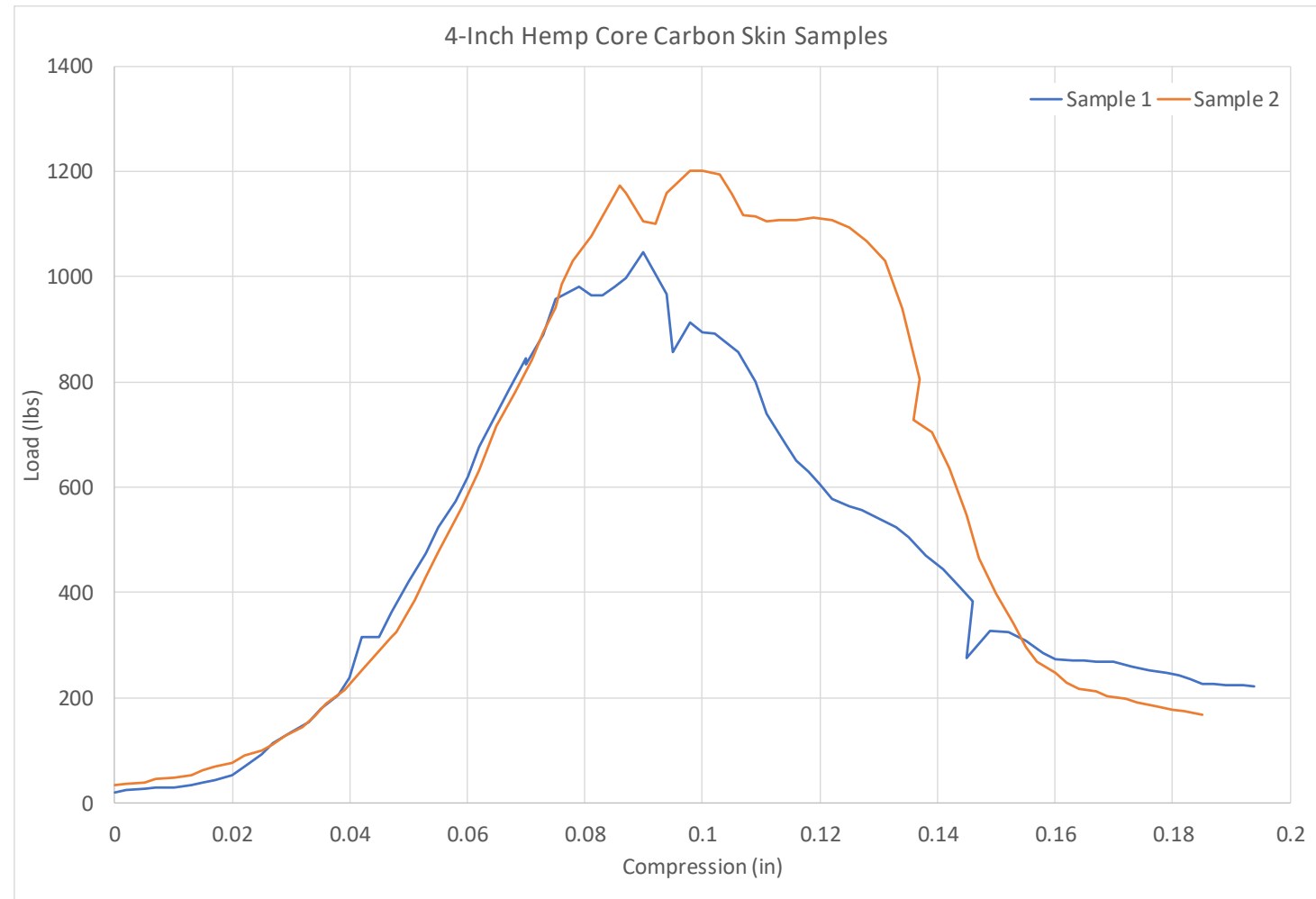

Figure 68: 4-Inch Hemp Core-Carbon Skin Load-Displacement Data 
The load-displacement curves for the 3-inch hemp core-carbon skin samples, seen in Fig. 68, display similar failure modes for the two samples but very different levels and a data capture error for the first sample. The curve seems to be truncated near the top, possibly at the point where buckling begins, and the sample no longer is truly axially loaded. As shown in Fig. 69, Sample 1 has buckled at the top, which resulted in an even lower ultimate load than Sample 2.

It is important to note that the buckling that the sample undergoes early in the test, and with both samples, causes a drastic decrease in both ultimate load and stiffness. It can be seen in the load-displacement graph for both samples that they still hold a high load far past the ultimate load. Sample 2 holds between 1100 and 1200 pounds for nearly .05 inches of compression, over $50 \%$ of the compression experienced before ultimate load.

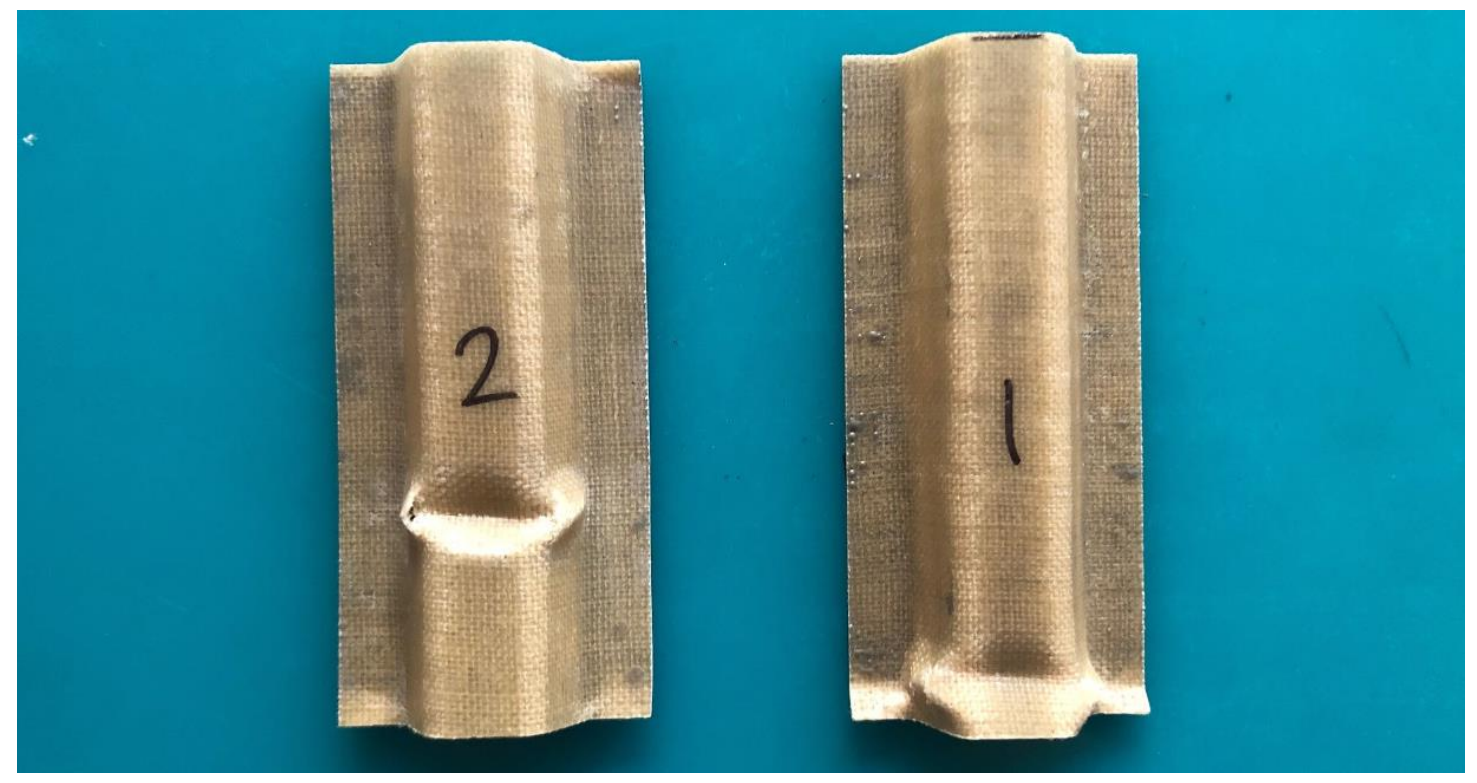

Figure 69: 4-Inch Hemp Core-Carbon Skin Compression Test Failed Samples 
The stiffnesses of the two samples were close, despite Sample 1 and Samples 2 failing in different ways. The two samples had large enough regions of linearity, even with the data capture error and truncation at the ultimate load, as shown in Fig. 70.

The average stiffness of the 4-inch hemp core-carbon skin samples was 25822 pounds per inch. This was a significant drop,

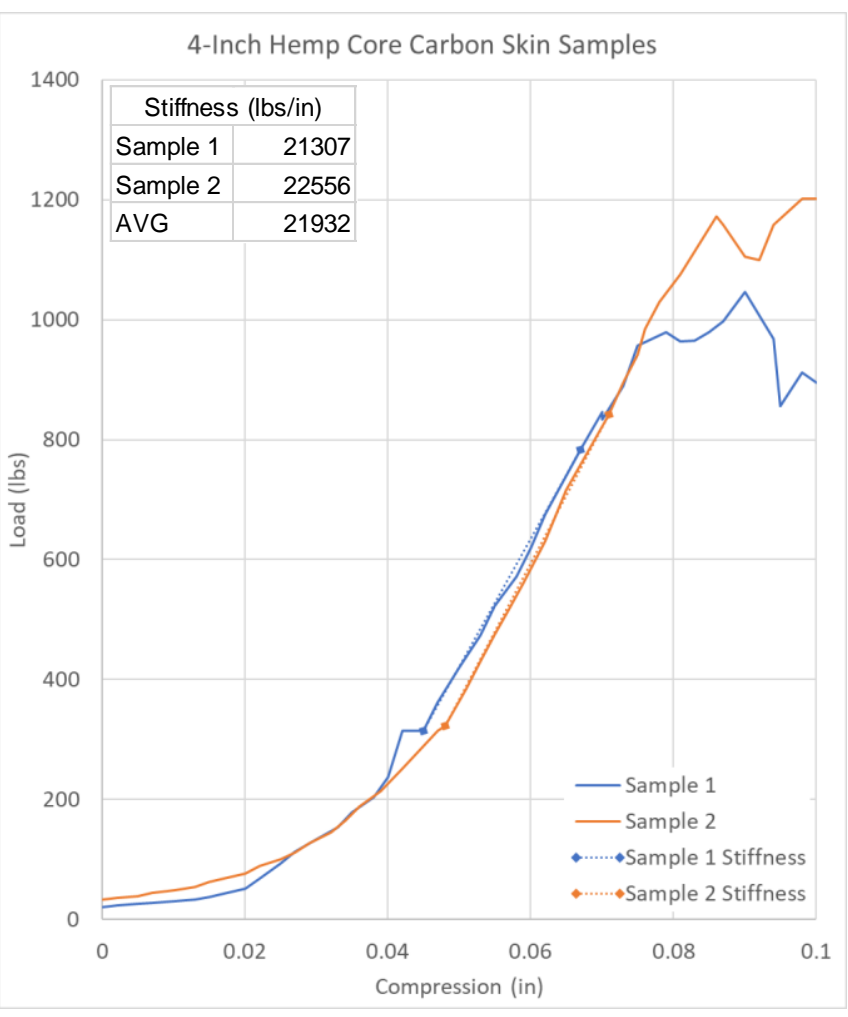

Figure 70: 4-Inch Hemp Core-Carbon Skin Stiffnesses

even from the trend for the hemp

core-carbon skin samples. This stiffness was very close to the 4-inch hemp fiber samples. The very low stiffnesses of these samples were again likely the result of the drastic global buckling behavior seen during the compression test. It is much more likely that the 1- and 2- inch samples are more representative of the stiffness of the structure in context of the application.

\subsection{Results Comparison and Discussion}

The goal of this research is to investigate whether bio-composites have benefits when added to a traditional composite corrugated structure. It has been demonstrated that bio-composites are much cheaper and more environmentally friendly, the degree of which 
can depend on the epoxy matrix used. Their strength in comparison to traditional composites, however, has been questioned. This created the desire to see if the benefits of both materials could be harnessed using this clever corrugated structure.

\subsubsection{Average Ultimate Load}

The investigation of this desire can be approached from several perspectives of the mechanical test results of each length and configuration. The first of which, the average ultimate load of each configuration, can be seen in Table 37. This table shows the configurations - carbon core-carbon skin, hemp core-hemp skin, carbon core-hemp skin, and hemp core-carbon skin - in columns from left to right.

Table 37: Average Ultimate Load of Different Composite Configurations and Lengths

\begin{tabular}{|r|r|r|r|r|r|}
\hline \multicolumn{6}{|c|}{ Average Ultimate Load (lbs) } \\
\hline L (in) & \multicolumn{1}{|c|}{ CC } & HH & \multicolumn{1}{l|}{ CH } & HC \\
\hline & 1 & 1114 & 1344 & 1196 & 1444 \\
\hline 2 & 1504 & 1233 & 1293 & 1274 \\
\hline 3 & 1384 & 1202 & 1183 & 1183 \\
\hline & 4 & 1363 & 1162 & 1390 & 1124 \\
\hline AVG & & $\mathbf{1 3 4 1}$ & $\mathbf{1 2 3 6}$ & $\mathbf{1 2 6 5}$ & $\mathbf{1 2 5 6}$ \\
\hline
\end{tabular}

From this we can see that, in general, the average ultimate load of the mixed samples was closer to that of the all-hemp fiber samples. The length of the samples did not seem to adhere to a trend, although the 1- and 2-inch samples held greater loads than the 3- and 4-inch. It should be noted again that at the 1-inch length, all samples outperformed the all-carbon fiber samples. 
This is thought to be due to the higher propensity of the longer samples to experience more extreme eccentric loading situations. The effect of unevenness after cutting, even when mitigated by the cardboard, is magnified by the length and thus greater moment arm. The shorter samples could experience local buckling and material failure, although the all-carbon samples experienced the lowest average ultimate load, likely due to the early debonding of the core and skin. When comparing to the all-carbon fiber and all-hemp samples, it is much clearer to look at the percentages of the control number, seen below in Tables 38 and 39.

Table 38: Average Ultimate Load Percent Differences Compared to All-Carbon

\begin{tabular}{|c|c|c|c|c|}
\hline \multicolumn{5}{|c|}{ Samples } \\
\hline \multicolumn{5}{|c|}{ Average Ultimate Load (Ibs) Compared to CC } \\
\hline $\mathrm{L}$ (in) & $\mathrm{CC}$ & $\mathrm{HH}$ & $\mathrm{CH}$ & $\mathrm{HC}$ \\
\hline 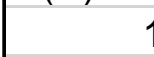 & $100 \%$ & $121 \%$ & $107 \%$ & $130 \%$ \\
\hline 2 & $100 \%$ & $82 \%$ & $86 \%$ & $85 \%$ \\
\hline 3 & $100 \%$ & $87 \%$ & $85 \%$ & $85 \%$ \\
\hline 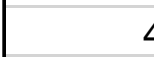 & $100 \%$ & $85 \%$ & $102 \%$ & $82 \%$ \\
\hline AVG & $100 \%$ & $91 \%$ & $93 \%$ & $92 \%$ \\
\hline
\end{tabular}

Table 39: Average Ultimate Load Percent Differences Compared to All-Hemp Samples

\begin{tabular}{|r|r|r|r|r|r|}
\hline \multicolumn{5}{|c|}{ Average Ultimate Load (lbs) Compared to $\mathrm{HH}$} \\
\hline L (in) & CC & HH & CH & HC \\
\hline 1 & $83 \%$ & $100 \%$ & $89 \%$ & $107 \%$ \\
\hline 2 & $122 \%$ & $100 \%$ & $105 \%$ & $103 \%$ \\
\hline 3 & $115 \%$ & $100 \%$ & $98 \%$ & $98 \%$ \\
\hline 4 & $117 \%$ & $100 \%$ & $120 \%$ & $97 \%$ \\
\hline AVG & $\mathbf{1 1 5} \%$ & $\mathbf{1 0 0} \%$ & $\mathbf{1 0 9} \%$ & $\mathbf{1 0 8 \%}$ \\
\hline
\end{tabular}


Of the mixed samples, only the 4-inch carbon core-hemp skin samples outperformed the same length of the all-carbon fiber samples, holding a 2 percent greater load. Overall, the hemp core-carbon skin samples held the second highest loads to the allcarbon fiber samples by just 4 percent.

As can be seen in Fig. 71, the samples with the hemp cores seemed to follow a distinct trend of decreasing average ultimate load while the samples with carbon cores seemed to be erratic. The all-carbon samples are denoted CC, the all-hemp samples are denoted $\mathrm{HH}$, the carbon core-hemp skin samples are denoted $\mathrm{CH}$, and the hemp corecarbon skin samples are denoted HC. Looking solely at the 1-inch samples is an indication of the bonding strength of the epoxy. This bonding strength seems to be exceeded by the forces experienced by the 1-inch samples with carbon cores whereas the samples with hemp cores did not experience bonding failure and held their full load. The 2-inch samples all did not experience bonding failure and seemed to have minimal global buckling. Global buckling, in addition to the local buckling seen in the 1- and 2-inch, had a large effect on the testing of the 3-and 4- inch samples, resulting in the diminished loads seen below. 


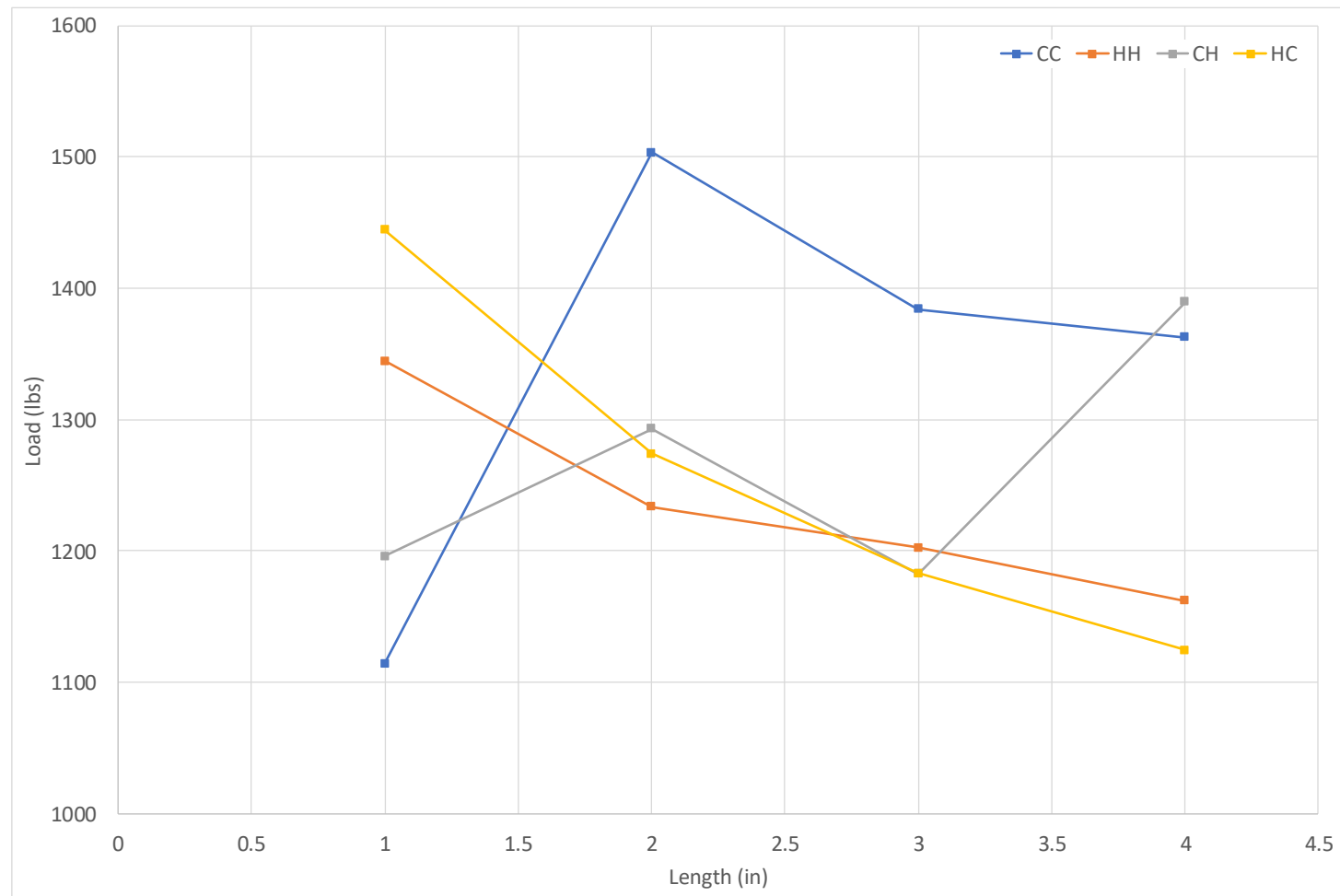

Figure 71: Average Ultimate Load Cross-Material and Cross-Length Comparison Plot

\subsubsection{Average Ultimate Stress}

The second approach to analyzing and comparing the results of each configuration is to look at the average ultimate stress held in each axial compression test. The stress was calculated by measuring and calculating the surface contact area of the sample. This stress was assumed for the structure, though the stress seen in each material would be different. As can be seen in Table 40, the mixed composite samples held nearly the same average ultimate stress as the all-carbon fiber sample, while the all-hemp fiber sample held a much lower stress across all lengths. With a thickness of 0.04 inches, three plies of hemp fiber were much thicker than the three-ply carbon laminate thickness of 0.018 . This resulted in the highest average ultimate stress for the all-carbon samples. 
Due to the bonding failure, however, the hemp core-carbon skin samples held the highest average ultimate stress of the 1-inch samples. This result was key, as the hypothesis that the integration of hemp into the structure prevented debonding was proven for the 1 inch case where debonding was a key flaw of the all-carbon samples. So, despite not reaching as high of an ultimate stress as some of the all-carbon fiber samples, the hemp core-carbon skin samples could reach the ultimate stress of the structure, rather than that of the bond.

Table 40: Average Ultimate Stress of Different Composite Configurations and Lengths

\begin{tabular}{|r|r|r|r|r|r|}
\hline \multicolumn{5}{|c|}{ Average Ultimate Stress (lbs/in^2) } \\
\hline L (in) & \multicolumn{1}{|c|}{ CC } & HH & CH & HC \\
\hline & 1 & 14527 & 7635 & 11435 & 10815 \\
\hline 2 & 19479 & 7071 & 10660 & 9474 \\
\hline 3 & 17958 & 6904 & 9838 & 8931 \\
\hline & 4 & 17758 & 6794 & 11538 & 8464 \\
\hline AVG & & $\mathbf{1 7 4 3 0}$ & $\mathbf{7 1 0 1}$ & $\mathbf{1 0 8 6 8}$ & $\mathbf{9 4 2 1}$ \\
\hline
\end{tabular}

Fig. 72 shows these data points and the disparity between the all-carbon samples and the other three material configurations in terms of stress. This disparity demonstrates the difference in thickness for the hemp fiber and the carbon fiber sections. Again, this stress is an approximation as it does not factor in thickness changes through the specimen, additional epoxy used in bonding, and loading differences due to different material stiffnesses. Although the mixed material stresses seem low, this is due to the thick hempfiber sections that add a large amount of cross-sectional area. While this stress is useful in analyzing the strength versus thickness, it does not account for the density of the material, nor the stress distribution in different parts of the structure. 


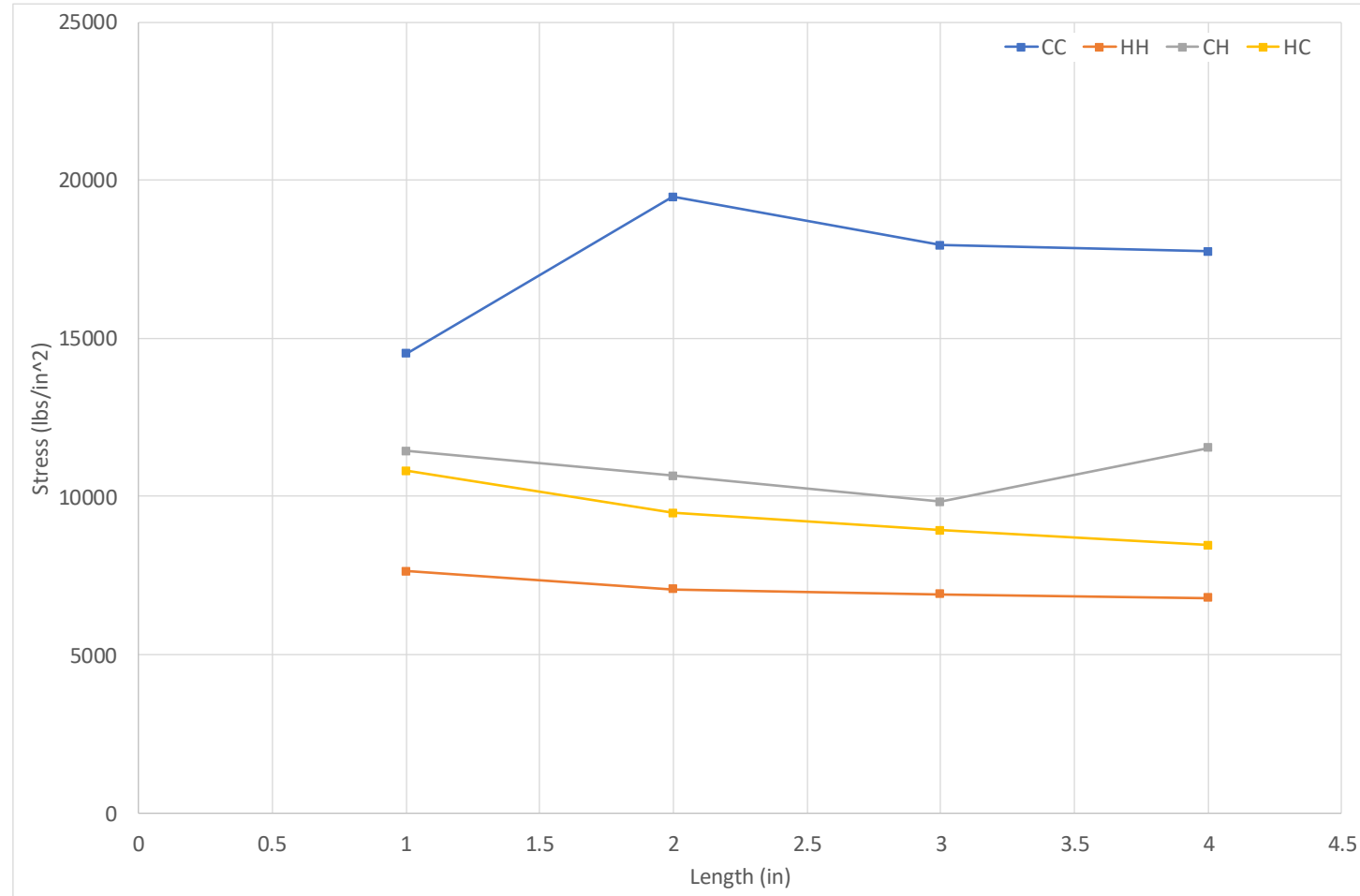

Figure 72: Average Ultimate Stress Cross-Material and Cross-Length Comparison Plot

\subsubsection{Average Stiffness}

The third approach to cross-analyzing the results of all cases and length is approximating the compressive stiffness of each case. On average, the hemp core-carbon skin samples had the highest overall stiffness, with the highest stiffness of all samples being the 1-inch sample of this configuration. Most samples predictably decreased in stiffness as length was increased, with the exception of the all-carbon fiber samples. These samples seemed to increase in stiffness as length was increased, but this was due to the debonding associated with the shorter length samples. The samples that incorporated hemp in some form showed better results as strength reduction due to debonding was mitigated. 
Table 41 shows the cross-material and cross-length comparison for average stiffness. The all-hemp samples had the lowest stiffness by a definitive margin at 25149 pounds per inch, as could be predicted, while the other three configurations all sat at around an average stiffness above 34000 pounds per inch.

Table 41: Average Stiffness of Different Composite Configurations and Lengths

\begin{tabular}{|c|c|c|c|c|}
\hline \multicolumn{5}{|c|}{ Average Stiffness (Ibs/in) } \\
\hline L (in) & $\mathrm{CC}$ & $\mathrm{HH}$ & $\mathrm{CH}$ & $\mathrm{HC}$ \\
\hline & 128810 & 28002 & 30664 & 49510 \\
\hline & 48072 & 21019 & 38528 & 35868 \\
\hline & 39126 & 21860 & 35434 & 36314 \\
\hline & 37043 & 21802 & 35563 & 21932 \\
\hline AVG & 38263 & 23171 & 35047 & 35906 \\
\hline
\end{tabular}

Looking at the percent difference between the all-carbon samples and the other samples, the hemp core-carbon skin samples had just a 3 percent lower stiffness averaged between all lengths and a very significant 72 percent higher stiffness at the 1-inch length. The carbon core-hemp skin and all-hemp samples had a 7 percent lower and 36 percent lower stiffness respectively at all lengths. These can be seen below in Table 42 .

Table 42: Average Stiffness Percent Differences Compared to All-Carbon Samples

\begin{tabular}{|r|r|r|r|r|r|}
\hline \multicolumn{6}{|c|}{ Average Stiffness (lbs/in) Compared to CC } \\
\hline L (in) & CC & HH & \multicolumn{1}{|c|}{ CH } & HC \\
\hline 1 & $100 \%$ & $97 \%$ & $106 \%$ & $172 \%$ \\
\hline 2 & $100 \%$ & $44 \%$ & $80 \%$ & $75 \%$ \\
\hline 3 & $100 \%$ & $56 \%$ & $91 \%$ & $93 \%$ \\
\hline 4 & $100 \%$ & $59 \%$ & $96 \%$ & $59 \%$ \\
\hline AVG & $100 \%$ & $\mathbf{6 3} \%$ & $\mathbf{9 5 \%}$ & $\mathbf{9 7 \%}$ \\
\hline
\end{tabular}


Table 43: Average Stiffness Percent Differences Compared to All-Hemp Samples

\begin{tabular}{|r|r|r|r|r|}
\hline \multicolumn{5}{|c|}{ Average Stiffness (lbs/in) Compared to $\mathrm{HH}$} \\
\hline L (in) & CC & HH & $\mathrm{CH}$ & $\mathrm{HC}$ \\
\hline 1 & $103 \%$ & $100 \%$ & $110 \%$ & $177 \%$ \\
\hline 2 & $229 \%$ & $100 \%$ & $183 \%$ & $171 \%$ \\
\hline 3 & $179 \%$ & $100 \%$ & $162 \%$ & $166 \%$ \\
\hline 4 & $170 \%$ & $100 \%$ & $163 \%$ & $101 \%$ \\
\hline AVG & $\mathbf{1 7 6} \%$ & $\mathbf{1 0 0} \%$ & $\mathbf{1 6 1} \%$ & $\mathbf{1 6 5 \%}$ \\
\hline
\end{tabular}

In comparison to the all-hemp samples, all other material configurations had higher stiffnesses. However, the hemp core-carbon skin samples only had a greater stiffness by 1 percent, indicating a greatly diminished stiffness due to the global buckling failure mode mentioned earlier.

Trends are even easier to see in Fig. 73, which plots these data points and shows the trend of each configuration. The all-carbon fiber samples increased in stiffness until leveling off at the 3-inch samples. The clearest trend in stiffness was held, again, by the hemp core-carbon skin samples, where average stiffness fell sharply but consistently. The carbon core-hemp skin samples, however, held a similar stiffness across all lengths but showed no clear trend. While the all-hemp samples had a comparable stiffness at the 1inch length with the carbon core-hemp skin samples, this stiffness sharply fell and stayed low for the 2- to 4-inch samples. 


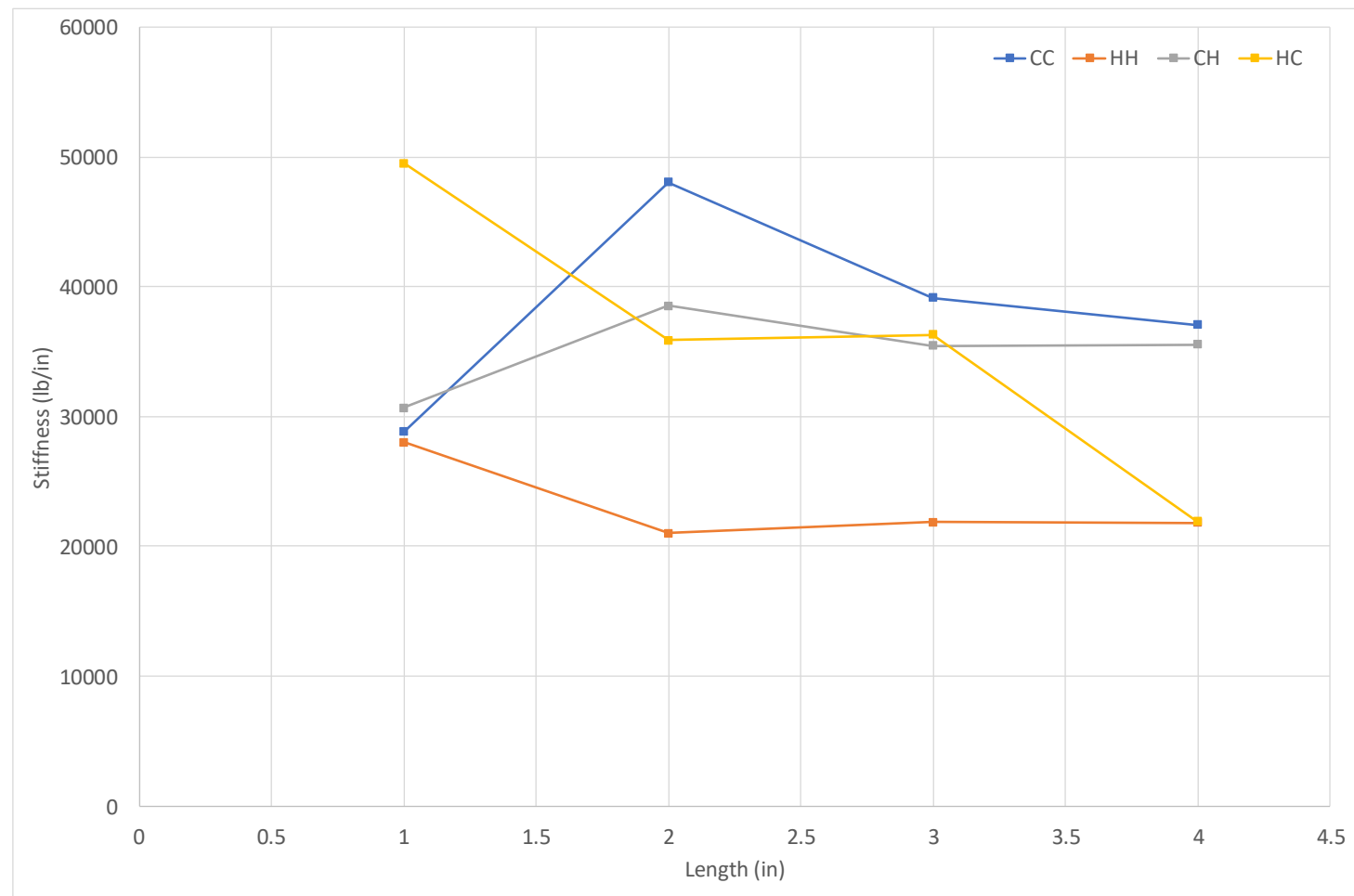

Figure 73: Average Stiffness Cross-Material and Cross-Length Comparison Plot

\subsubsection{Weight Adjusted Average Ultimate Load}

The final way these results were interpreted was by analyzing the average strengthtoo-weight ratio of each sample. This was done by dividing the ultimate load in compression in pounds that the sample withstood by the weight of the sample in pounds. This was done to normalize the samples and compensate for the increased thickness - and likely resin content - of the hemp fiber skins and cores. This is a relevant measure for aerospace applications where weight is the first and foremost concern. Strength to weight ratio is by far the greatest strength of fully carbon fiber parts, especially in corrugated structures and sandwich panels. 
This held true throughout all tested lengths, as the very low weight of the carbon fiber samples resulted in the highest ratios of ultimate load to sample weight. However, the samples with integrated hemp fiber parts held loads somewhat well, especially at the shorter lengths. These values can be seen in Table 44 .

Table 44: Average Ultimate Load-Over-Weight of Composite Configurations and Lengths

\begin{tabular}{|c|c|c|c|c|}
\hline \multicolumn{5}{|c|}{ Average Ultimate Load (Ibs)/Weight of Sample(Ibs) } \\
\hline L (in) & $\mathrm{CC}$ & $\mathrm{HH}$ & $\mathrm{CH}$ & $\mathrm{HC}$ \\
\hline 1 & 259175 & 195446 & 210703 & 2312 \\
\hline 2 & 192119 & 85412 & 113527 & 1032 \\
\hline 3 & 110789 & 56420 & 69213 & 6288 \\
\hline 4 & 82694 & 40548 & 64326 & 45736 \\
\hline AVG & 161194 & 94457 & 114442 & 11076 \\
\hline
\end{tabular}

\subsubsection{Failure Mode Discussion}

When discussing the validity of the comparative results shown previously, it is important to consider the failure modes of each sample configuration and length, outside of the ideal axial crushing mode. These failure modes are numerous and varied in the experimental results of this research. The failure modes that were seen include debonding of the skin from the core, bearing stress resulting in edge splintering, end rolling, local face buckling, and global buckling.

Each of these failure modes will be discussed further in the following sections, along with the consequences each failure mode had on the experiment and the results of that specific configuration. 


\subsubsection{Debonding}

Debonding was one failure mode that was of interest in this experiment specifically in which cases it occurs and how it can be prevented. In this experiment, the only samples where this was obviously and consistently seen were the 1 -inch all-carbon fiber samples. Two of the samples where this is most prevalent, samples 1 and 3, can be seen below in Fig. 74. This was in line with previous research and hypotheses stemming from it. Being that one of the goals of this research is to determine if differing the material properties in the different parts of the corrugated structure can solve this issue, the debonding failure mode was a very valid result. This failure mode always resulted in highly diminished ultimate load and stiffness.

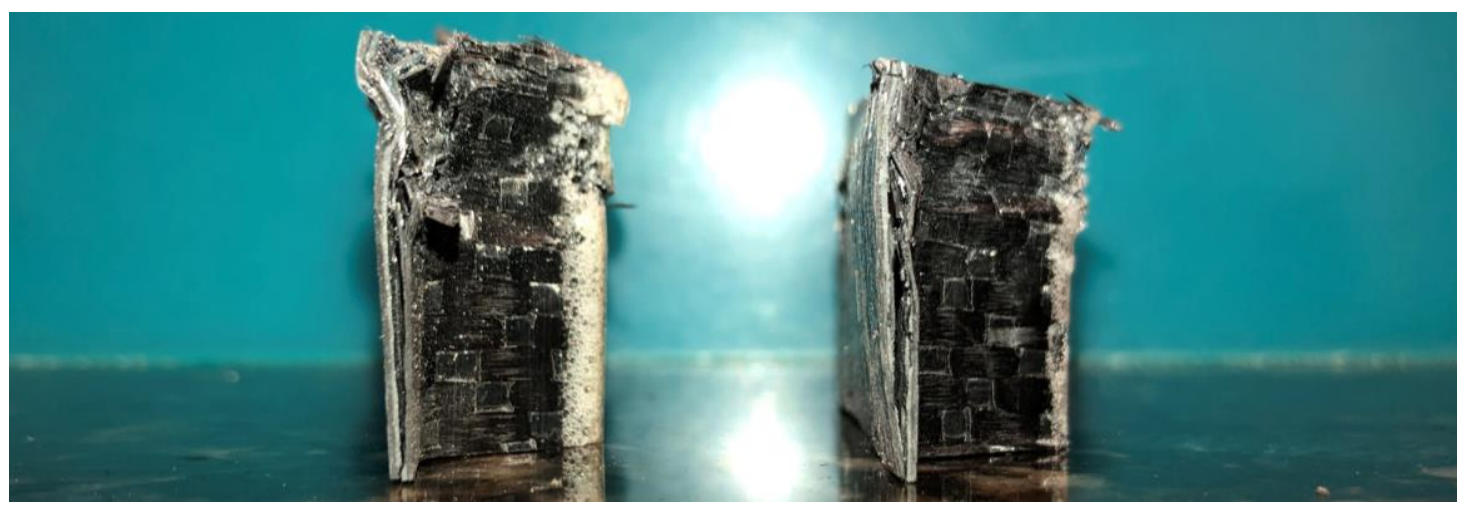

Figure 74: 1-Inch All-Carbon Fiber Samples 1 and 3 with Skin-Core Debonding Failure

The likely cause of debonding was the equal global buckling of both the skin and the core away from each other, as can be clearly seen in sample 1 . The tension imparted by this buckling on the bonding epoxy overcomes the strength of this bond and the two faces separate. The equal stiffnesses of the carbon fiber skin and core likely intensifies this force 
while replacing one with hemp allows the structure to stay adhered. With the bond intact, the structure still leverages the sandwich structure and has a much higher ultimate load and stiffness, although the adhered skin and core flange still buckles. This can be seen in Fig. 75, where samples 1 and 2 of the 1 -inch hemp core-carbon skin configuration can be seen still fully adhered.

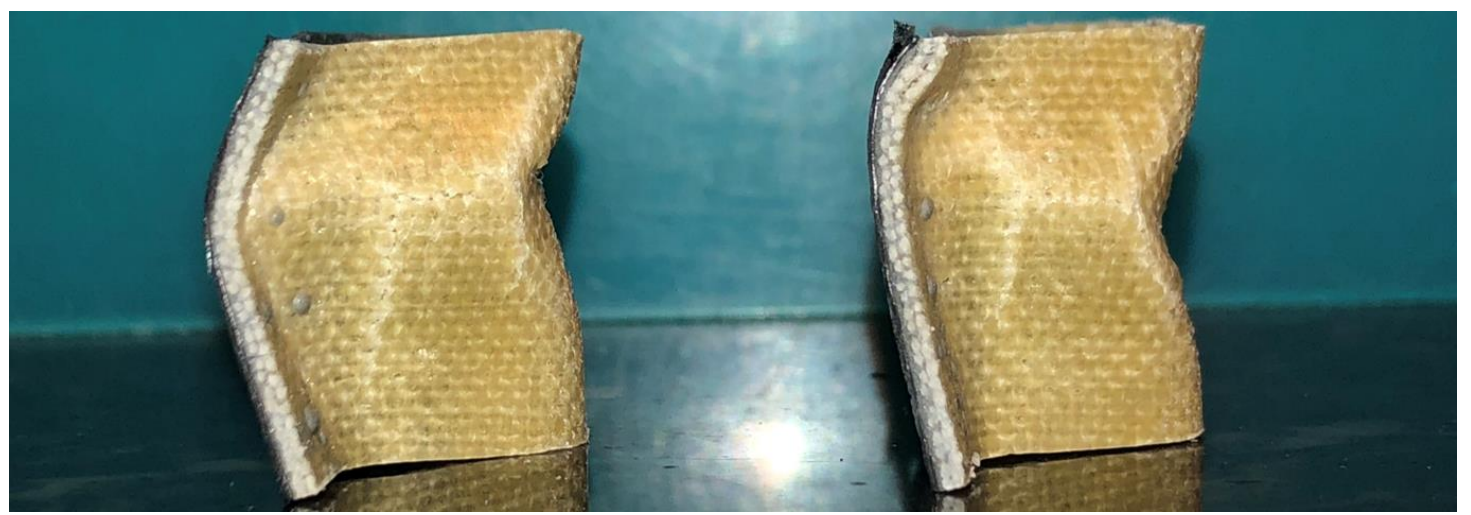

Figure 75: 1-Inch Hemp Core-Carbon Skin Samples 1 and 2 without Skin-Core Debonding

\subsubsection{Bearing Stress}

Bearing stress is defined as the contact pressure between two bodies, in the case of this experiment the two bodies being the sample and the test fixture. The failure mode was seen in many of the samples with a carbon fiber core, as the cross-section of that component was very thin. This resulted in splintering, which can again be seen in the 1 -inch all-carbon samples seen to the in Fig. 76. This failure mode was seen in many of the all-carbon fiber samples as well as many of the carbon core-hemp fiber samples, although to a lesser degree. 
Failure as a result of this stress

did not seem to be a main factor in reduced strength or stiffness. However, this failure mode, in combination with end rolling, was sometimes responsible for global buckling, which had a very large impact on both ultimate load and stiffness.

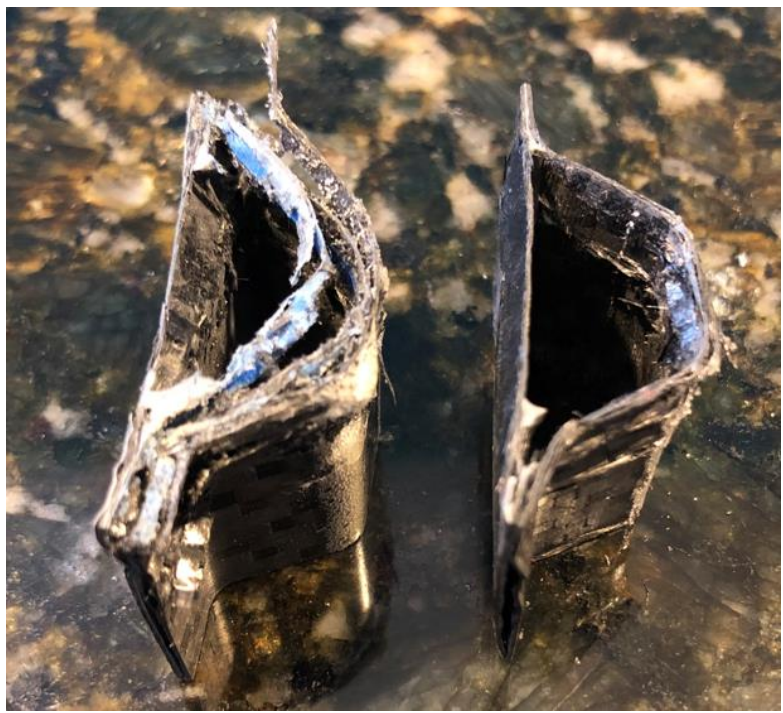

Figure 76: 1-Inch All-Carbon Fiber Samples with Bearing Stress Failure

\subsubsection{Local Buckling}

Of the two types of buckling failure modes seen during this experiment, local buckling can be differentiated as buckling of individual faces of the test samples. This failure mode was often seen in samples with a hemp core like those seen in Fig. 77 and 78. This mode was also primarily seen in the shorter 1- and 2-inch samples. With these samples, bearing stress and debonding problems were solved with the integration of hemp, as discussed before. As a result, the structure itself was pushed to failure, coming to an ultimate load before it was unable to deform axially and was forced to buckle. Due to the area moment of inertia of the cross-section and the short length of the material, these samples were very stable and avoided the unfavorable global buckling failure mode.

Samples failing due to local buckling saw some of the highest loads at their length, making this the most desired failure mode. Samples also held high loads for sustained periods after ultimate load, another desirable characteristic of this failure mode. 


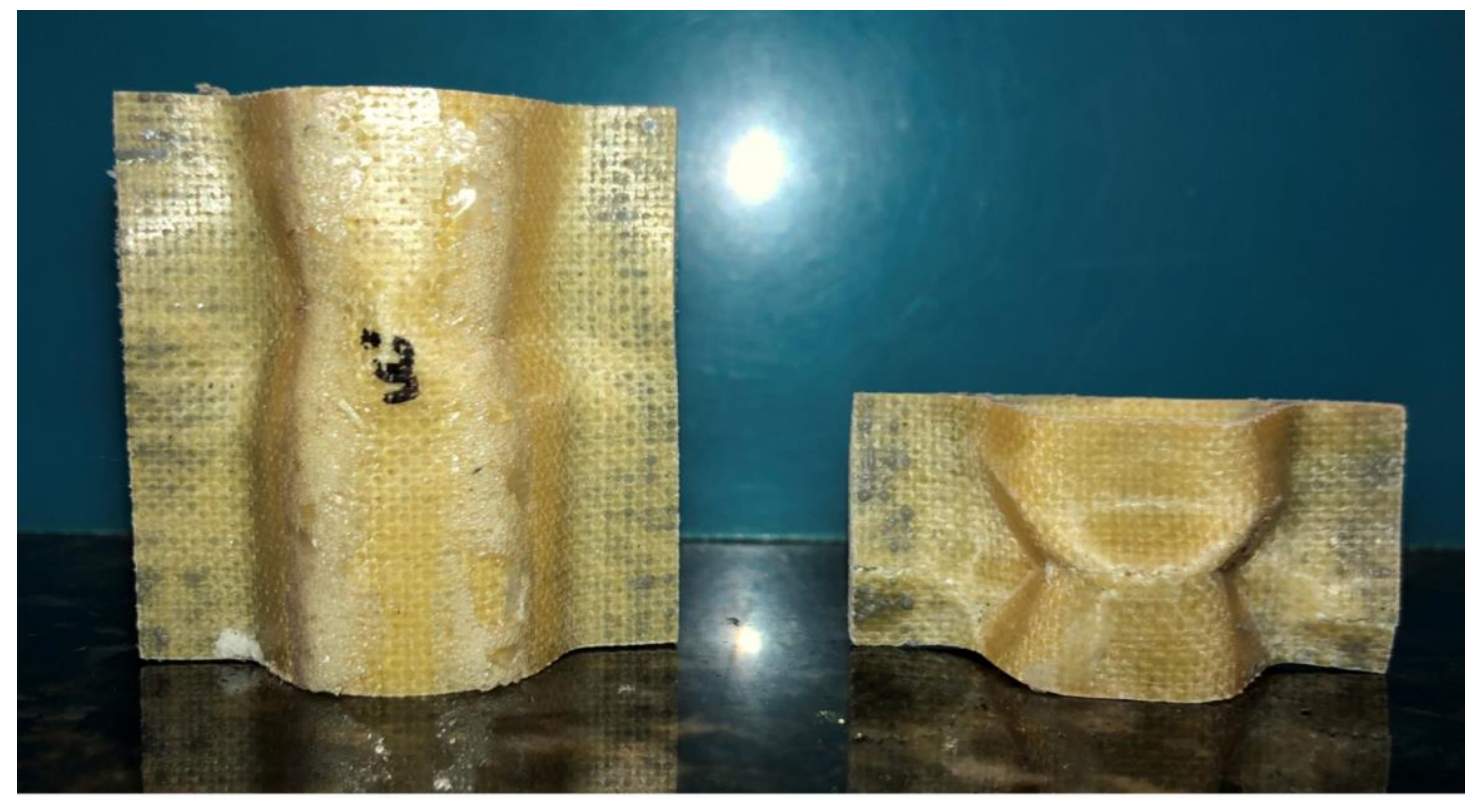

Figure 77: 2-Inch and 1-Inch All-Hemp Samples 3 and 1 With Local Buckling

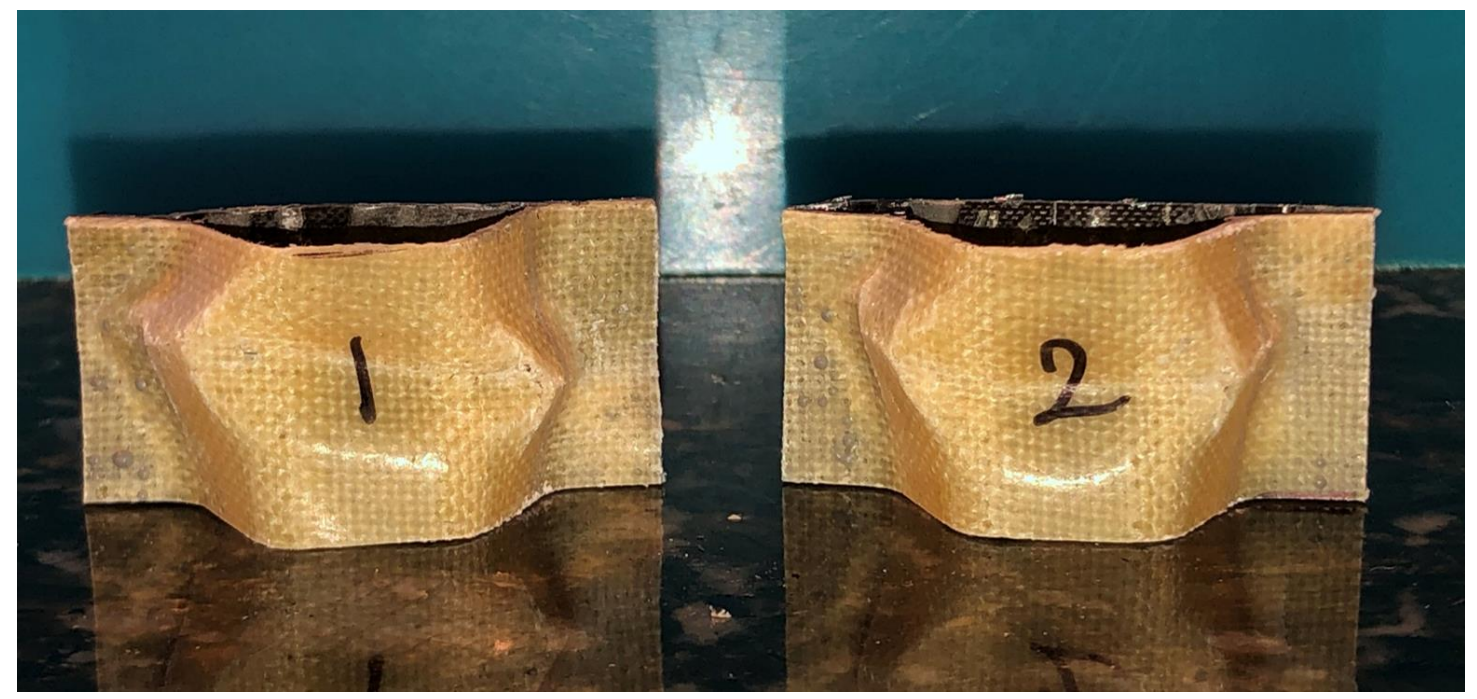

Figure 78: 1-Inch Hemp Core-Carbon Skin Samples 1 and 2 with Local Buckling 
A combination of the bearing stress failure mode and local buckling was seen in many of the longer samples - at 3 and 4 inches where eccentricity was intensified in the loading configuration. This causes the end of the sample to curl over, although the rest of the shape of the structure is mostly preserved. This failure mode was appropriately called end rolling. Samples with this failure mode, like the two carbon core-hemp skin samples seen in Fig.

80, had somewhat diminished

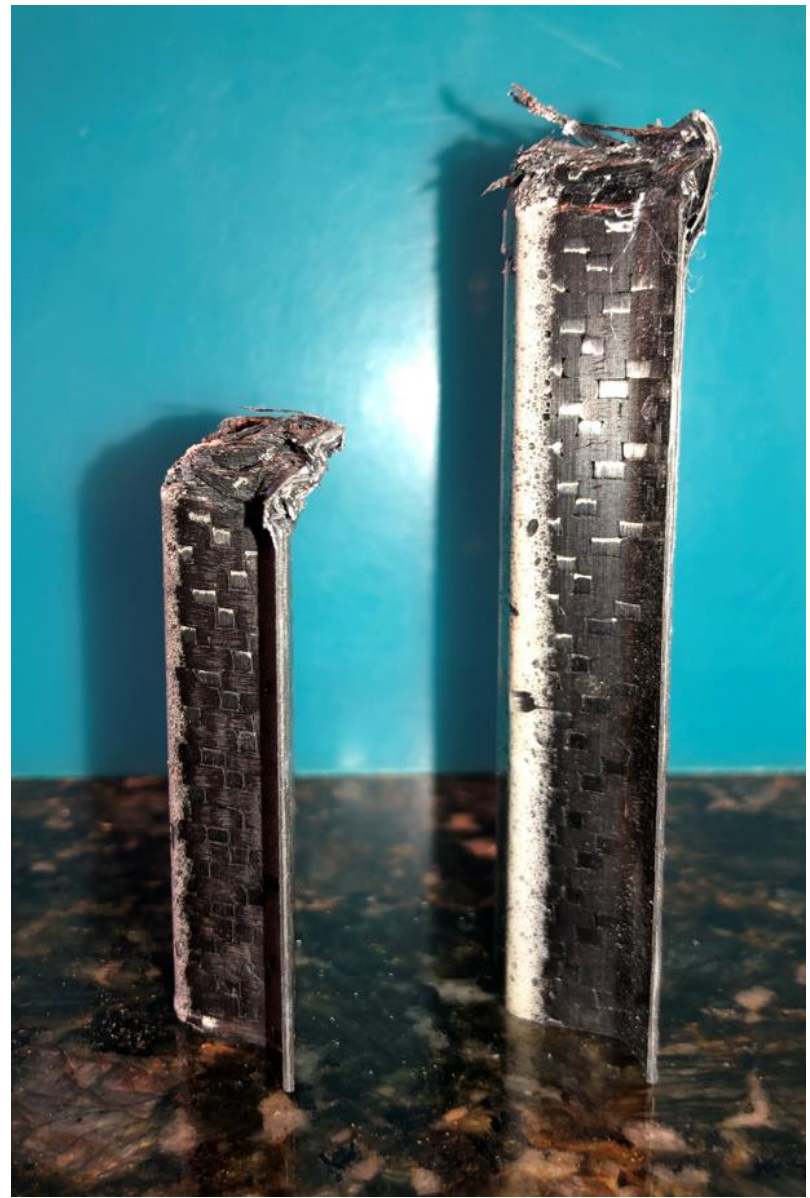

Figure 79: 4- and 3-Inch All-Carbon Samples with Local Buckling and Bearing Stress strength and stiffness.

However, the main impact of this behavior was the post-failure extension of high loads in the load-displacement curve. This is due to the overall structure avoiding damage as only the top or bottom is damaged. This creates a new contact surface for the load to be applied to. This was not a desirable failure mode, although the strength of the material was not impacted as much as in global buckling behavior - the other failure mode widely seen in the 3- and 4-inch samples. 

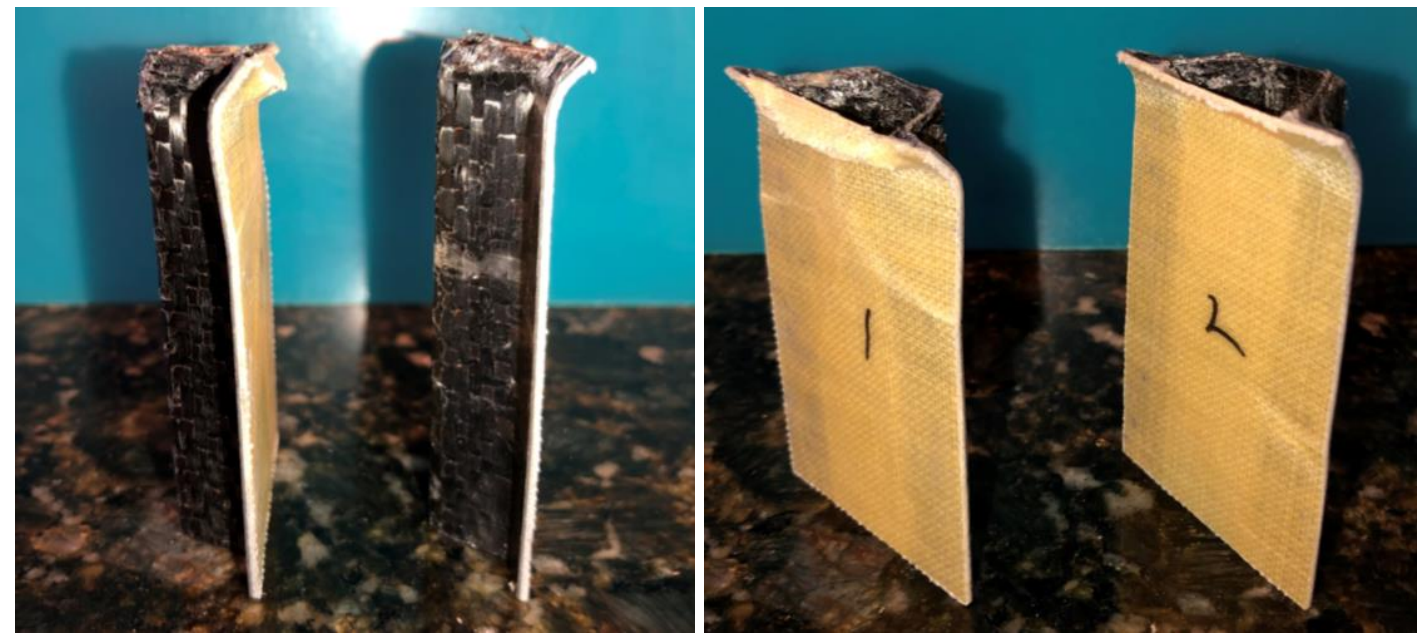

Figure 80: 3-Inch Carbon Core-Hemp Skin Samples with Local Buckling and Bearing Stress

\subsubsection{Global Buckling}

Global buckling was the most prevalent and most problematic of this experiment, although at the onset was expected due to the asymmetrical and unstable structure of the samples. This failure mode can be differentiated from local buckling in that it is a buckling of the entire structure, as opposed to individual faces. This was especially seen in the 4-inch samples with a hemp core, although seen in many of the 3- and 4-inch samples to varying degrees.

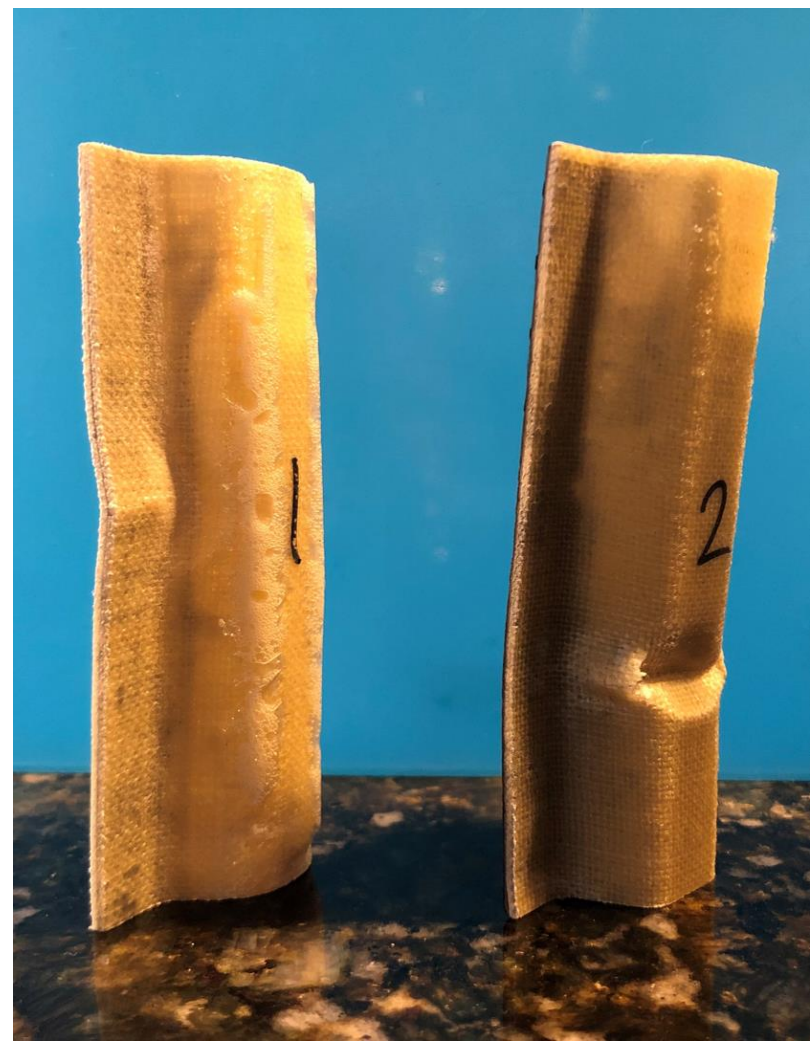

Figure 81: 4-Inch All-Hemp and Hemp CoreCarbon Skin Samples with Global Buckling 
Samples undergoing this failure mode, like those seen in Fig. 81, had both greatly diminished ultimate loads and stiffnesses. This is due to the compromise of the entire structure and the applicable material properties changing. As opposed to axial strength being tested, the bending resistance of the structure is now tested, again often with the corrugation compromised.

While this failure mode was expected in longer lengths, the large impact of global buckling on results for those sample lengths brings with it the discussion of how to crossanalyze these results with those of the shorter samples. The longer samples exaggerate the buckling propensity of these samples but are not as relevant to the real-world application of the sandwich panel as the shorter samples. This is mainly due to the stability of the shorter samples, as in application, corrugated sandwich panels are unlikely to be a singular, long cell. Rather many cells, creating a stable plate. This makes axial compression testing a single cell at a short length a good representative test of the material properties while testing the longer length provides a worst-case buckling scenario.

For this reason, it could be considered more valid to discuss the 1- and 2-inch lengths together as representative of the same kind of edgewise test. This also is due to the similar and relevant failure modes seen in the 1- and 2-inch samples - most notably the lack of global buckling and end rolling. 


\subsubsection{Adjusted Results Tables}

When discussing the validity of the comparative results shown previously, it is important to consider the failure modes of each sample configuration and length. These failure modes are numerous and varied but, after discussing each of them and their position within the scope of this research, it was concluded that a more relevant way of analyzing the final results would be to group lengths that shared similar failure modes.

The shorter lengths, seen below in Tables 45 and 46, the average ultimate load and average stiffness respectively, demonstrated failure modes more in line with pure and stable axial compression testing. The crushing and local buckling behavior seen in these samples allowed an analysis of a representative section of a full size and stable corrugated sandwich panel. This was true even for the 1-inch all-carbon sample. This sample demonstrated that a low bonding surface area and incompatible skin-core stiffness relationship would ultimately result in debonding and a drastically diminished ultimate load and stiffness. Overall, at these shorter lengths, the hemp core-carbon skin samples had highest ultimate load by at least 4 percent, considering the high mass of the all-hemp samples. These samples also had a 10 percent higher stiffness than the all-carbon samples and were much stiffer still than the carbon core-hemp skin and all-hemp samples. 
Table 45: 1- and 2-Inch Ultimate Load of Different Composite Configurations

\begin{tabular}{|l|r|r|r|r|r|}
\hline \multicolumn{5}{|c|}{ Average Ultimate Load (lbs) } \\
\hline L (in) & \multicolumn{1}{|l|}{ CC } & HH & \multicolumn{1}{l|}{ CH } & HC \\
\hline 1 & 1114 & 1344 & 1196 & 1444 \\
\hline & 2 & 1504 & 1233 & 1293 & 1274 \\
\hline AVG & 1309 & 1289 & 1245 & 1359 \\
\hline AVG \% & $\mathbf{1 0 0 \%}$ & $\mathbf{9 8 \%}$ & $\mathbf{9 5 \%}$ & $\mathbf{1 0 4 \%}$ \\
\hline
\end{tabular}

Table 46: 1- and 2-Inch Stiffness of Different Composite Configurations

\begin{tabular}{|c|c|c|c|c|}
\hline \multicolumn{5}{|c|}{ Average Stiffness (lbs/in) } \\
\hline L (in) & $\mathrm{CC}$ & $\mathrm{HH}$ & $\mathrm{CH}$ & $\mathrm{HC}$ \\
\hline 1 & 28810 & 28002 & 30664 & 49510 \\
\hline 2 & 48072 & 21019 & 38528 & 35868 \\
\hline AVG & 38441 & 24511 & 34596 & 42689 \\
\hline AVG \% & $100 \%$ & $64 \%$ & $90 \%$ & $111 \%$ \\
\hline
\end{tabular}

While this method of analysis still has some uncertainties, it is much more valid than comparing all lengths of all samples, as some material combinations underwent much more drastic buckling at longer lengths, severely and misleadingly impacting the overall ultimate load and stiffness averages. 


\section{NUMERICAL ANALYSIS AND RESULTS}

Along with the experimental edgewise compression test, numerical analysis was used to simulate the compression test. This took the form of finite element analysis using ANSYS Workbench 19.2 Academic. Each test case and length were input using DesignModeler, the native CAD program in Workbench, along with material properties and the correct boundary conditions. ANSYS Workbench was the chosen numerical analysis program due to both the composites capabilities of the program, the ease of learning in comparison to other programs, and the availability of the program and suites.

\subsection{ANSYS Composites PrePost Procedure}

The setup of the framework for using ANSYS Composites

PrePost is based off a setup used in a CAE Associates Inc. seminar. In this, a traditional analysis setup is placed between ACP Pre and ACP Post. It is important to note which sections are routed to each other, as seen in Fig. 83. ACP Post has useful ply and thread failure tools but it was not used for this study. After

\begin{tabular}{|c|c|c|c|c|}
\hline \multicolumn{5}{|c|}{ Outline of Schematic A2, D2, E2: Engineering Data } \\
\hline & A & B & c & D \\
\hline 1 & Contents of Engineering Data & 0 & $(x$ & Source \\
\hline 2 & I Material & & & \\
\hline 3 & $\begin{array}{l}\text { Epoxy Carbon Woven (230 GPa) } \\
\text { Prepreg }\end{array}$ & $\nabla$ & $\square$ & 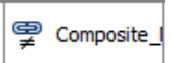 \\
\hline 4 & $\begin{array}{l}\text { Epoxy Carbon Woven (230 GPa) } \\
\text { Wet }\end{array}$ & 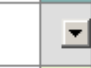 & $\square$ & 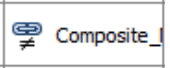 \\
\hline 5 & (1) Jalen's Hemp & $\nabla$ & $\square$ & 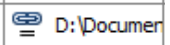 \\
\hline 6 & Jalen's Hemp 2 & 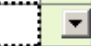 & $\square$ & $\stackrel{\text { ⿷⿱宀八 }}{=} \mathrm{D}:$ Documer \\
\hline 7 & Structural Steel & $\nabla$ & $\square$ & 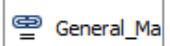 \\
\hline 8 & West System $105 / 206$ & 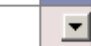 & $\square$ & 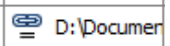 \\
\hline * & Click here to add a new material & & & \\
\hline
\end{tabular}

\begin{tabular}{|c|c|c|}
\hline \multicolumn{3}{|c|}{ Properties of Outline Row 6: Jalen's Hemp 2} \\
\hline & A & B \\
\hline 1 & Property & Value \\
\hline 2 & 7 Material Field Variables & 圈 Table \\
\hline 3 & 7 Density & 0.073985 \\
\hline 4 & 丹踞 Orthotropic Elasticity & \\
\hline 14 & Ð Tsai-Wu Constants & \\
\hline 18 & Ð Ұ Ply Type & \\
\hline
\end{tabular}

Figure 82: Material Properties Datasheet this framework is setup, the first 
step is selecting which materials should be included or created in engineering data. For the hemp, a combination of tested and researched material properties was used.

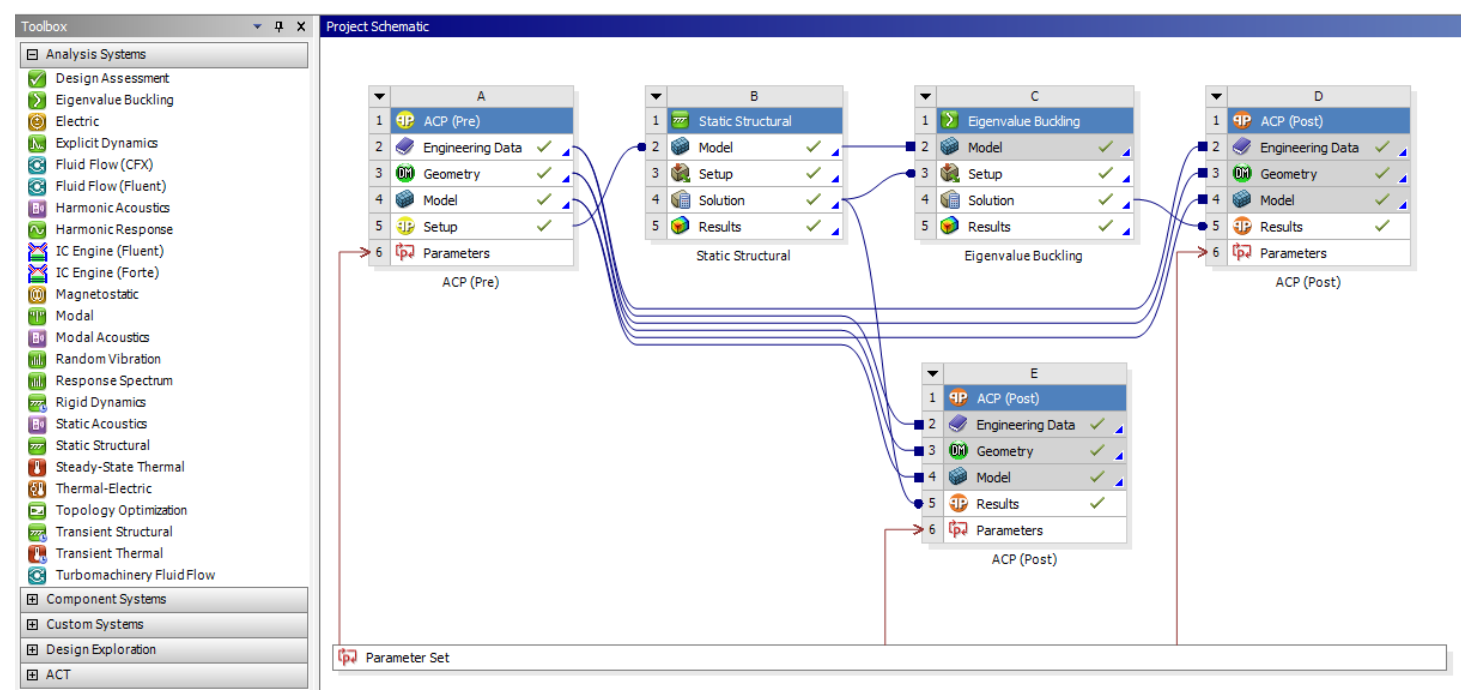

Figure 83: ANSYS Composites PrePost Static Structural and Eigenvalue Buckling Setup

While eigenvalue buckling was intended for use in this research, it was determined that the buckling seen in the experiment was too complex and varied to quantify with limited material properties available. However, this can be used with less difficulty in simpler geometries.

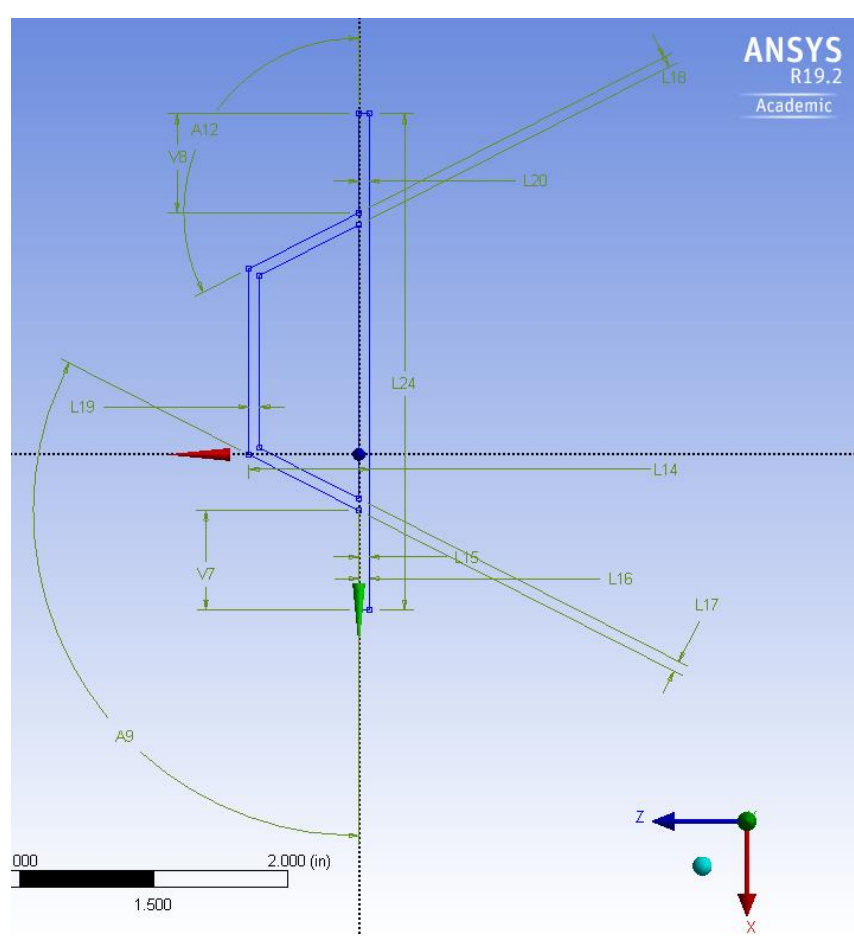

Figure 84: Hemp cross-section DesignModeler sketch view 
The second step in modeling the composite test samples in ANSYS was to step into DesignModeler and sketch the sample cross section. This was done using the measured thicknesses and angles from the physical test samples. This can be seen in Fig. 84. These thicknesses were changed for each different material case. This shape was then extruded to the correct sample length.

After this shape was extruded, the surface/skin tool was used to create a surface where the core and the skin each started. This was done in order to use ANSYS Composites Pre (ACP). This tool uses surfaces to lay up plies and allows for certain failure criteria to be placed. The surfaces can be seen in Fig. 85 .

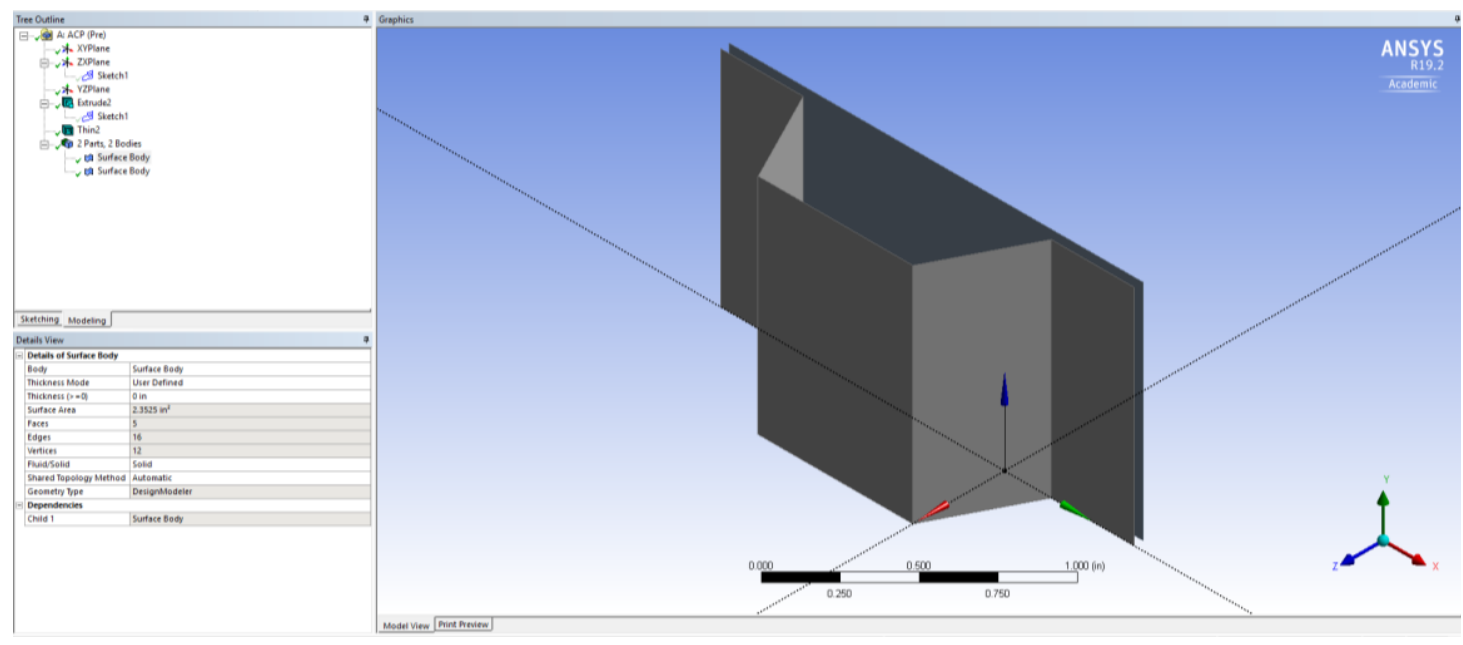

Figure 85: Extrude and Thin of Sketch 


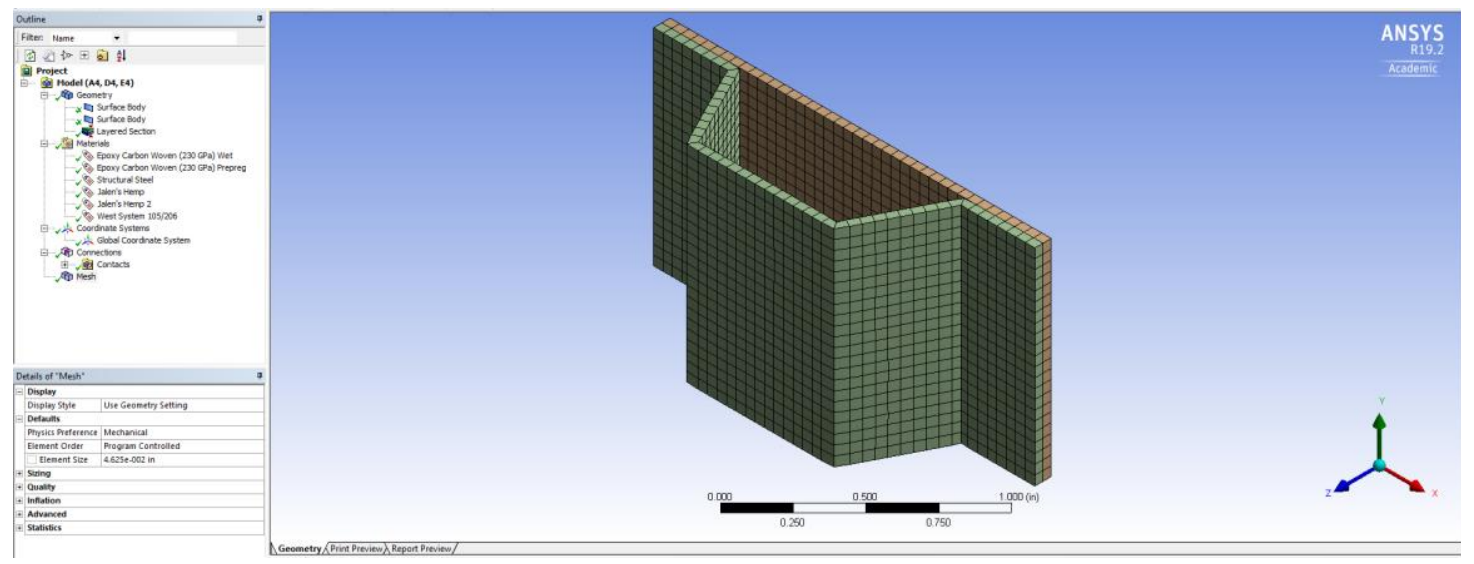

Figure 86: Meshed Surfaces in Model Step of ACP Pre

In the Model step of ACP Pre, a layered section is applied to these surface bodies. The thicknesses of these layered sections should correspond to the thicknesses of the hemp and carbon fiber cores and skins. These layered sections are then meshed, with hand calculations done to ensure that mesh is uniform and that the nodes of the inside trapezoid edges line up with nodes on the skin for node merging. Then this model is pushed into ACP Pre Setup.

Once open, ACP Pre Setup displays the surfaces used in the previous modeling phase. It also shows all of the material data sets. The first step in this phase is to create a fabric using one of the materials and assigning the thickness of one ply. For the all-hemp sample, the hemp material properties and thickness of one ply was used. This can be seen below in Fig. 87. 


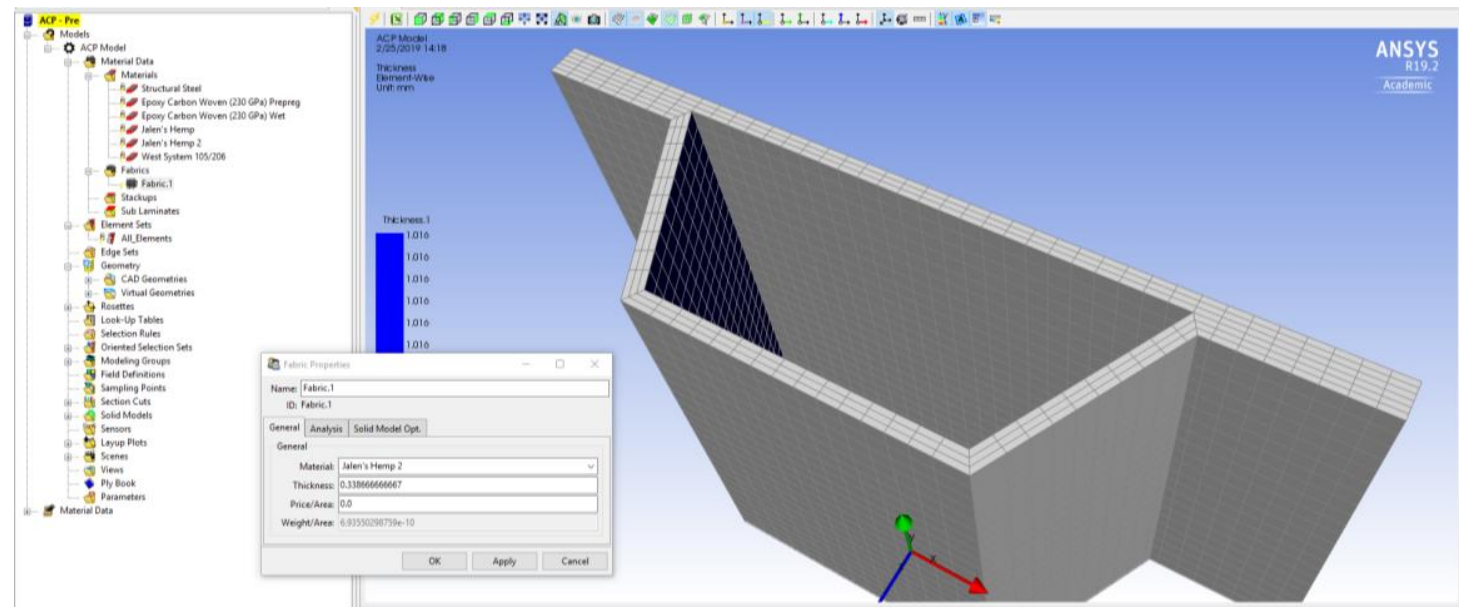

Figure 87: Assigning Material and Thickness to a Fabric in ACP Pre Setup

Then, an element set was created by selecting all elements. This was used to create an oriented selection of all elements, indicating towards which direction the composite plies will be laid up. It is important to note that the orientation requires a reference angle from a rosette, so one was created that uses the same reference directions as the original coordinate axis. These reference directions for ply lay-up can be seen in purple in Fig. 88.
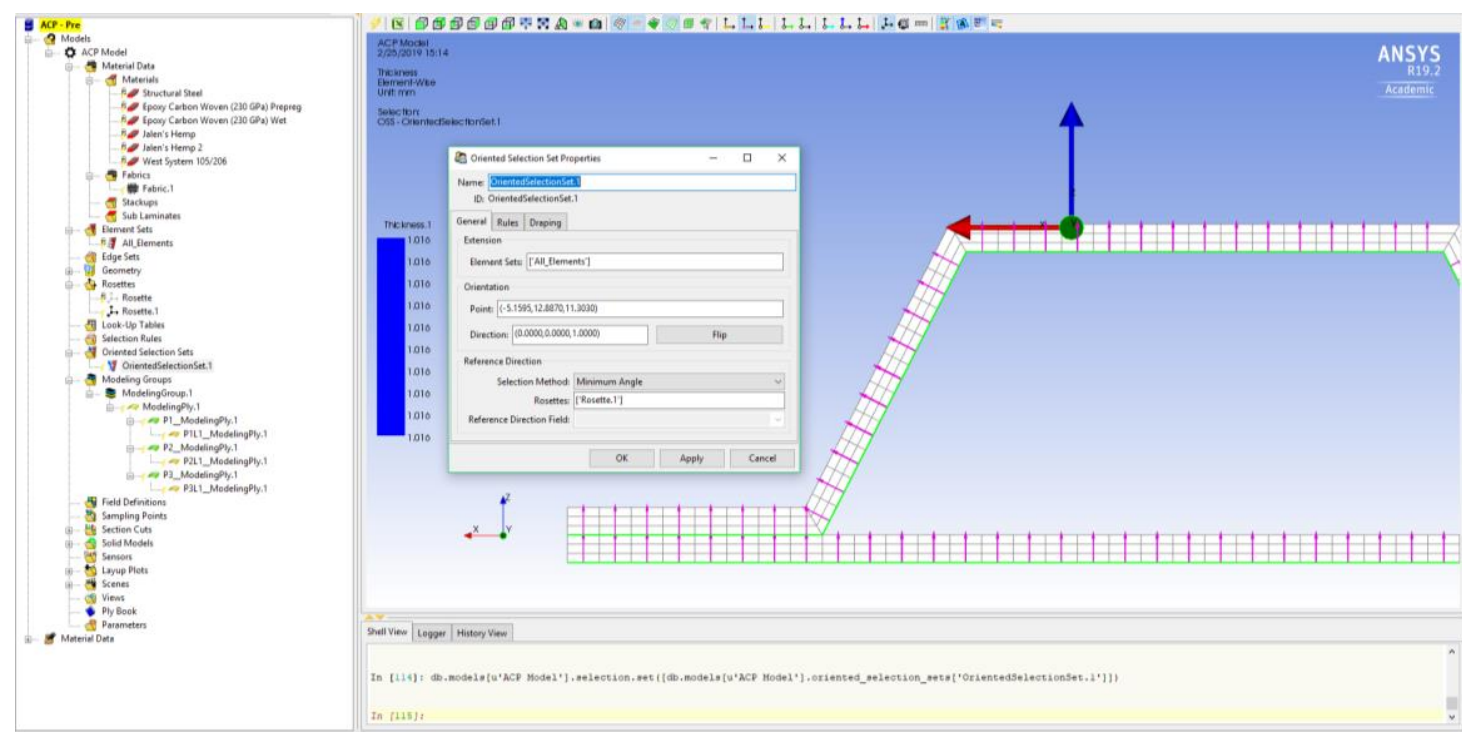

Figure 88: Oriented Selection Set Direction and Three Plies Laid Up from Green Surface 
After this, it was time to make the modeling ply group and then the first modeling ply type. When creating a modeling ply in the modeling group, the modeling ply properties must be set. In this case, the oriented selection set of all elements and the fabric that were created earlier were selected with the correct ply angle and number of layers. As can be seen in Fig. 89, the production ply properties list the correct material and thickness in millimeters per ply. Once the mesh had been updated, the ACP Pre step of this analysis was finished.

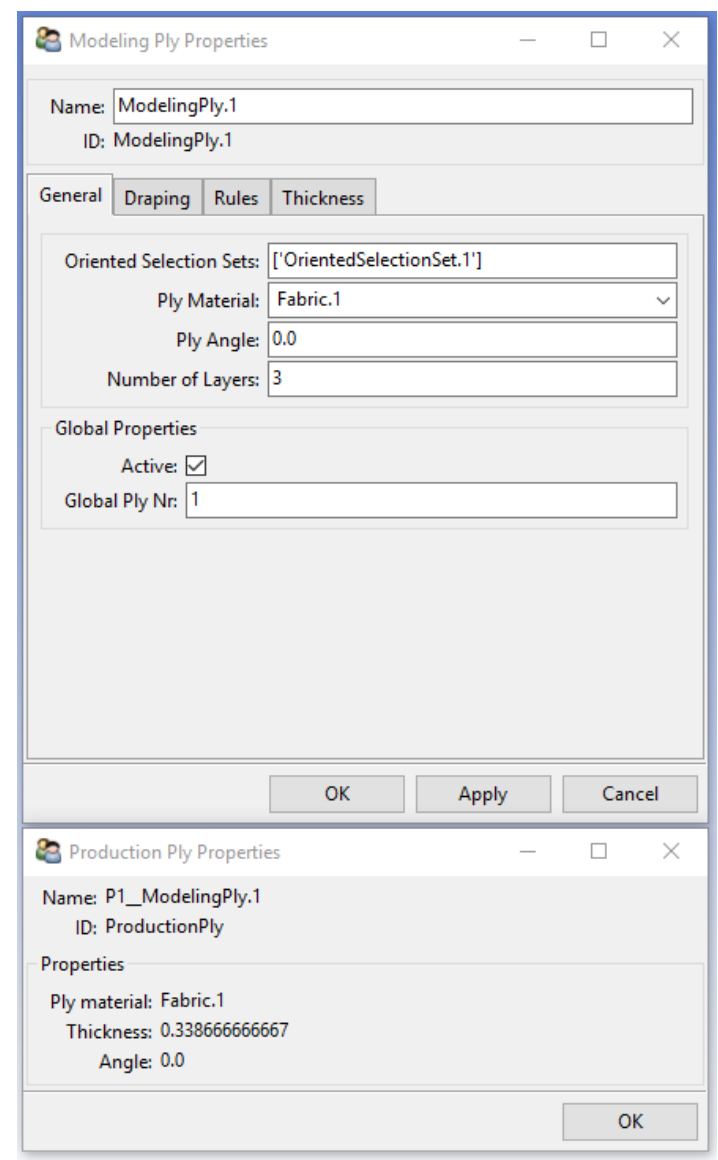

Figure 89: Modeling Ply Configuration in Modeling Group 


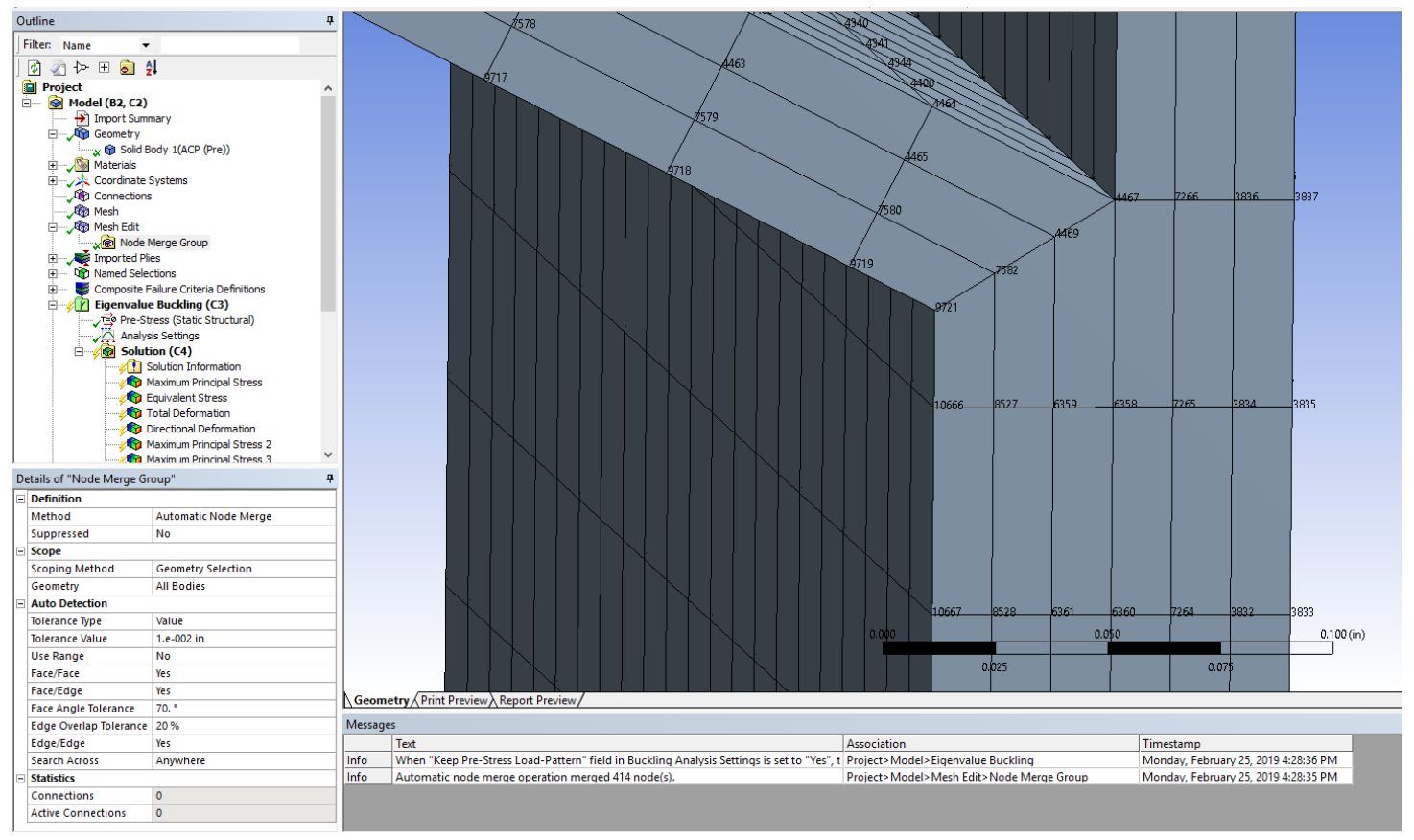

Figure 90: Node Numbers after Node Merge Group

After ACP Setup has been routed to the modeling step of the static structural analysis, the model and mesh from ACP Setup should be carried over. However, the skin and the core were still not bonded. To bond these two together, the node merge group tool was used to merge all overlapping nodes at the core-skin interface. For this, it was important to select a tolerance value that would merge the nodes at this interface without merging nodes on the same element together. To check this, the node numbers were displayed. If the node was not merged correctly, it displayed two node numbers as opposed to one, as seen above in Fig. 90. The correct number of merged nodes was also calculated from the geometry and node size and checked against the number of nodes that were automatically merged by the operation. For the one-inch all-hemp samples, this number was 414 nodes as each of the two flanges had an interface of 23 nodes by 9 nodes. 


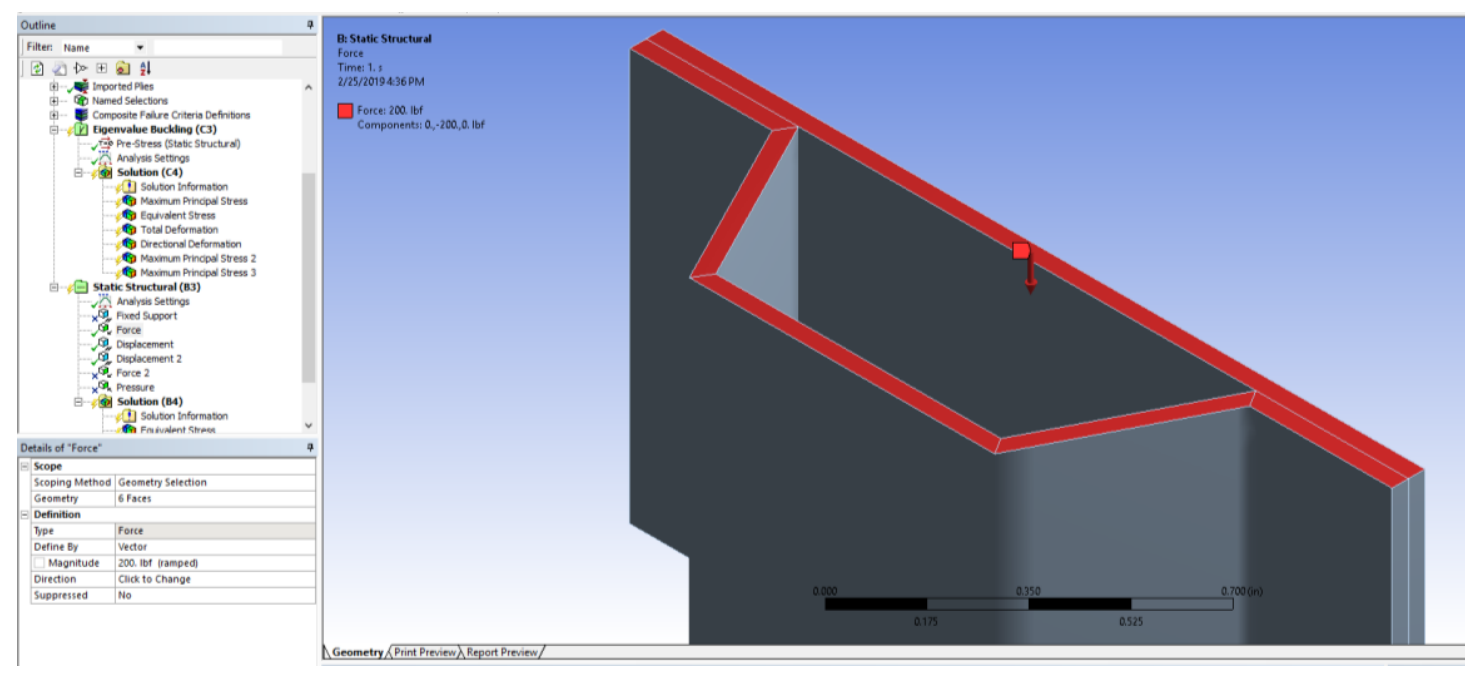

Figure 91: Surface Geometry Selected in Force Application

In the last step before solving, the force and boundary conditions were set. For the force, the entire top surface was selected and a load of 200 pounds was prescribed. This load, seen in Fig. 91, was selected because it was consistent for most samples and occurred just before local buckling in this sample. After this, the boundary conditions were set using displacement constraints. The first constraint allowed nodes on the top surface to only move in the vertical direction. This constraint models the vertical movement of the Instron crosshead and assumes that the friction of the sample holds the surface in place in the lateral directions. The second constraint allowed no movement at all across the bottom surface. This, again, assumes friction holds the surface completely still.

Finally, the test with the desired output parameters is run. In this case, it was the directional displacement on the vertical axis, as the goal was to find the error in displacement between this numerical analysis and experimental results. The results for this case can be seen in Fig. 92. 


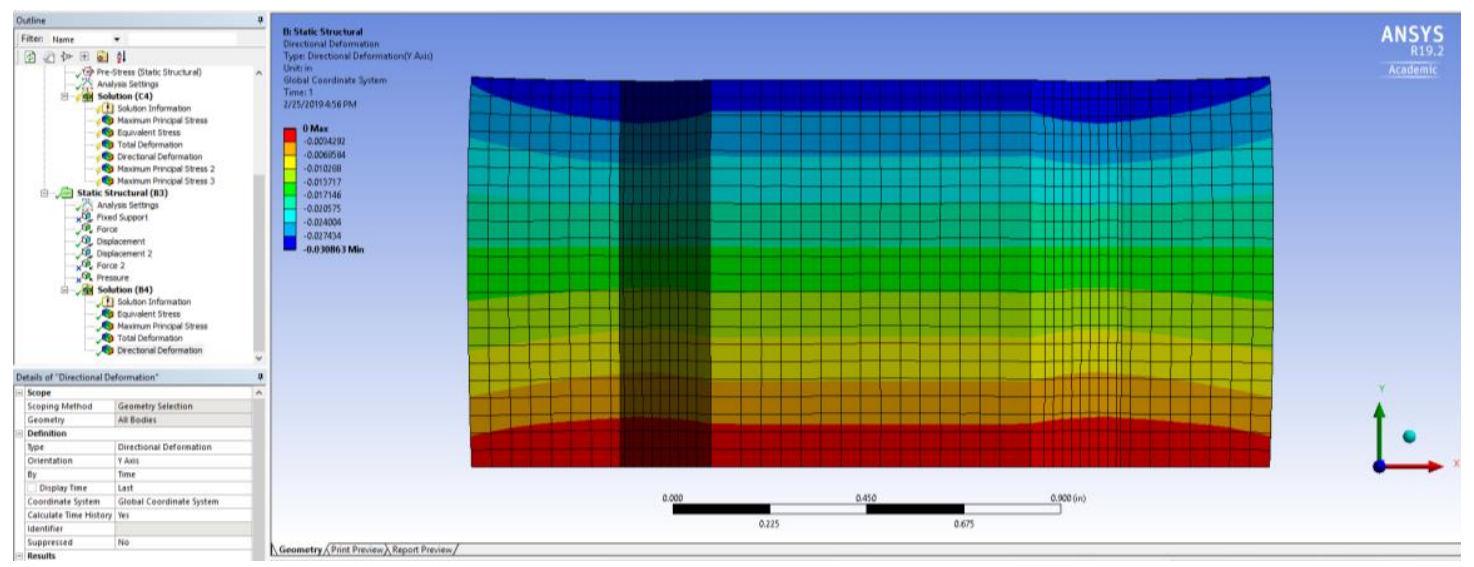

Figure 92: Surface Geometry Selected in Force Application

\subsection{ANSYS Results}

In order to attain relevant numerical results in ANSYS, suitable material properties must be used. For this case, due to the varying failure modes and the difficulty of modeling buckling in finite element programs, convenient material properties were taken from various sources. For the hemp material properties, very short compression tests were performed to get Young's modulus. This can be seen in Fig. 93. The slope of the mean stress-strain curve for this test was taken to get the

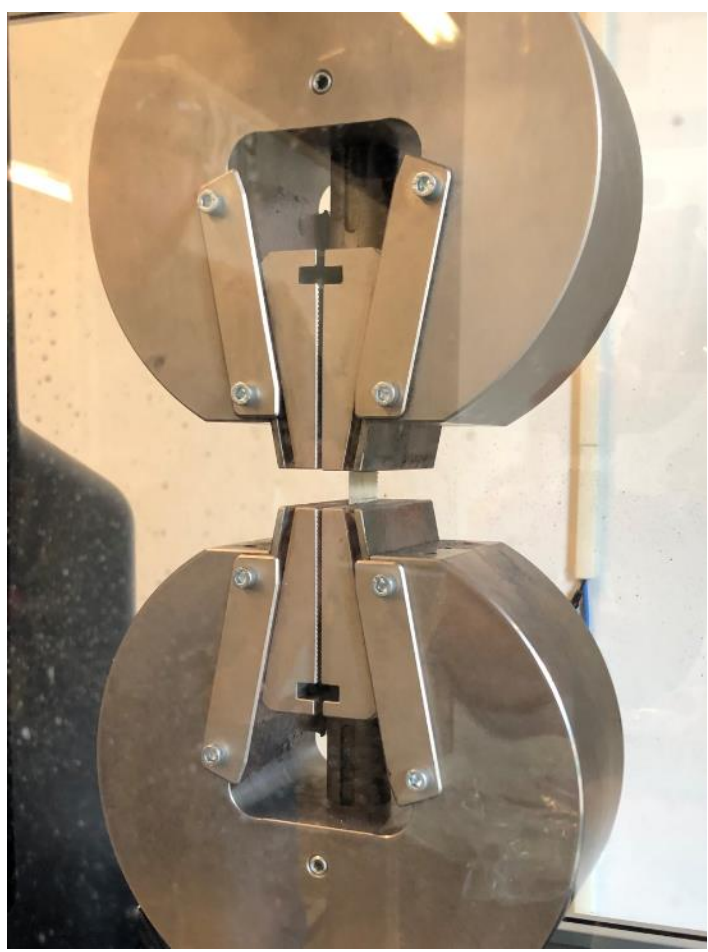

Figure 93: Hemp Material Property Compression Test

Young's Modulus. This sample buckled very early due to the instability of the test sample, so the slope was taken for the region right before buckling occurred, shown in Fig. 94. These slopes gave an average modulus of around 37500 pounds per square inch. 


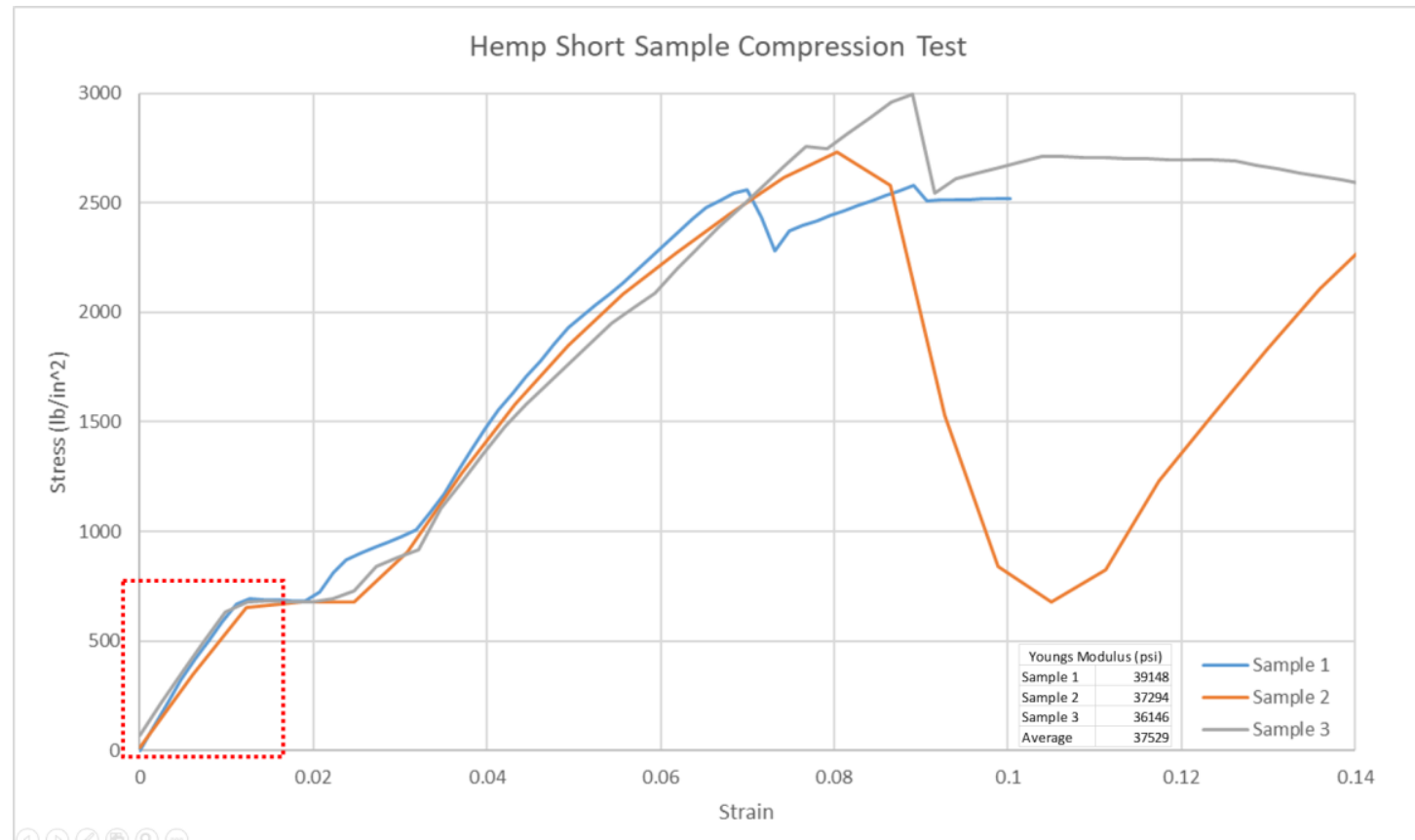

Figure 94: Hemp Compression Test for Young's Modulus (Pre-Buckling Region Outlined in Red)

For Poisson's ratio and shear modulus, values were taken from research done on the material properties of a very similar hemp weave. For the carbon fiber material properties, the HTA $51313 \mathrm{k} /$ Epocast $52 \mathrm{~A} / \mathrm{B}$ plain weave fabric wet layup was used from the Composites Materials Handbook, Volume 2. This material was used because it was one of the few wet layups in the handbook, and the density and ply thickness of $1.46 \mathrm{~g} / \mathrm{cc}$ and .00787 inches were close to the experimental density and ply thickness of $1.447 \mathrm{~g} / \mathrm{cc}$ and .006 inches. A summary of these material properties can be seen in Tables 47 and 48 . While the hemp, under compression, is modeled as an isotropic material for the sake of simplicity, it is important the material properties are input as one would an orthotropic material, for ANSYS Composites Pre. 
Table 47: Hemp ANSYS Material Properties

\begin{tabular}{|c|l|l|l|}
\hline \hline 1 & \multicolumn{1}{|c|}{ Property } & \multicolumn{1}{|c|}{ Value } & \\
\hline 2 & Z Material Field Variables & 圈 Table & \\
\hline 3 & T Density & 0.073985 & lb in^-3 \\
\hline 4 & $\square \quad$ Q Orthotropic Elasticity & & \\
\hline 5 & Young's Modulus X direction & 37500 & $\mathrm{psi}$ \\
\hline 6 & Young's Modulus Y direction & 37500 & $\mathrm{psi}$ \\
\hline 7 & Young's Modulus Z direction & 37500 & $\mathrm{psi}$ \\
\hline 8 & Poisson's Ratio XY & 0.19 & \\
\hline 9 & Poisson's Ratio YZ & 0.19 & \\
\hline 10 & Poisson's Ratio XZ & 0.19 & \\
\hline 11 & Shear Modulus XY & $1.0864 \mathrm{E}+08$ & $\mathrm{psi}$ \\
\hline 12 & Shear Modulus YZ & $1.0864 \mathrm{E}+08$ & $\mathrm{psi}$ \\
\hline 13 & Shear Modulus XZ & $1.0864 \mathrm{E}+08$ & $\mathrm{psi}$ \\
\hline
\end{tabular}

Table 48: Carbon Fiber ANSYS Material Properties

\begin{tabular}{|c|l|l|l|}
\hline 1 & \multicolumn{1}{|c|}{ Property } & \\
\hline 2 & 7 Material Field Variables & 圈 Table & \\
\hline 3 & 7 Density & 0.052259 & lb in^-3 \\
\hline 4 & $\square \quad$ Orthotropic Elasticity & & \\
\hline 5 & Young's Modulus X direction & $8.37 \mathrm{E}+06$ & $\mathrm{psi}$ \\
\hline 6 & Young's Modulus Y direction & $8.37 \mathrm{E}+06$ & $\mathrm{psi}$ \\
\hline 7 & Young's Modulus Z direction & $2.0925 \mathrm{E}+06$ & $\mathrm{psi}$ \\
\hline 8 & Poisson's Ratio XY & 0.04 & \\
\hline 9 & Poisson's Ratio YZ & 0.3 & \\
\hline 10 & Poisson's Ratio XZ & 0.3 & $\mathrm{psi}$ \\
\hline 11 & Shear Modulus XY & $6.74 \mathrm{E}+05$ & $\mathrm{psi}$ \\
\hline 12 & Shear Modulus YZ & $6.74 \mathrm{E}+05$ & $\mathrm{psi}$ \\
\hline 13 & Shear Modulus XZ & $1.04 \mathrm{E}+05$ & \\
\hline
\end{tabular}

From these material properties, the static structural simulation was run using ANSYS Composites Pre, as stepped through in the previous section. Directional displacement readings were taken to compare to the displacement at a certain applied force - in this case 200 pounds force - where consistent, linear behavior is still occurring and none of the varied failure modes have affected the load-displacement curve yet. 
At this point, the average value for displacement at 200 pounds was taken from each of the samples and compared to the ANSYS result for maximum directional deformation, seen above in Fig. 95. For the boundary conditions, again, the bottom surface was fixed in all directions and the top surface was fixed in the lateral directions, with nodes only able to move vertically. To find the displacement at 200 pounds in the experimental results, linear interpolation was used with the load-displacement data.

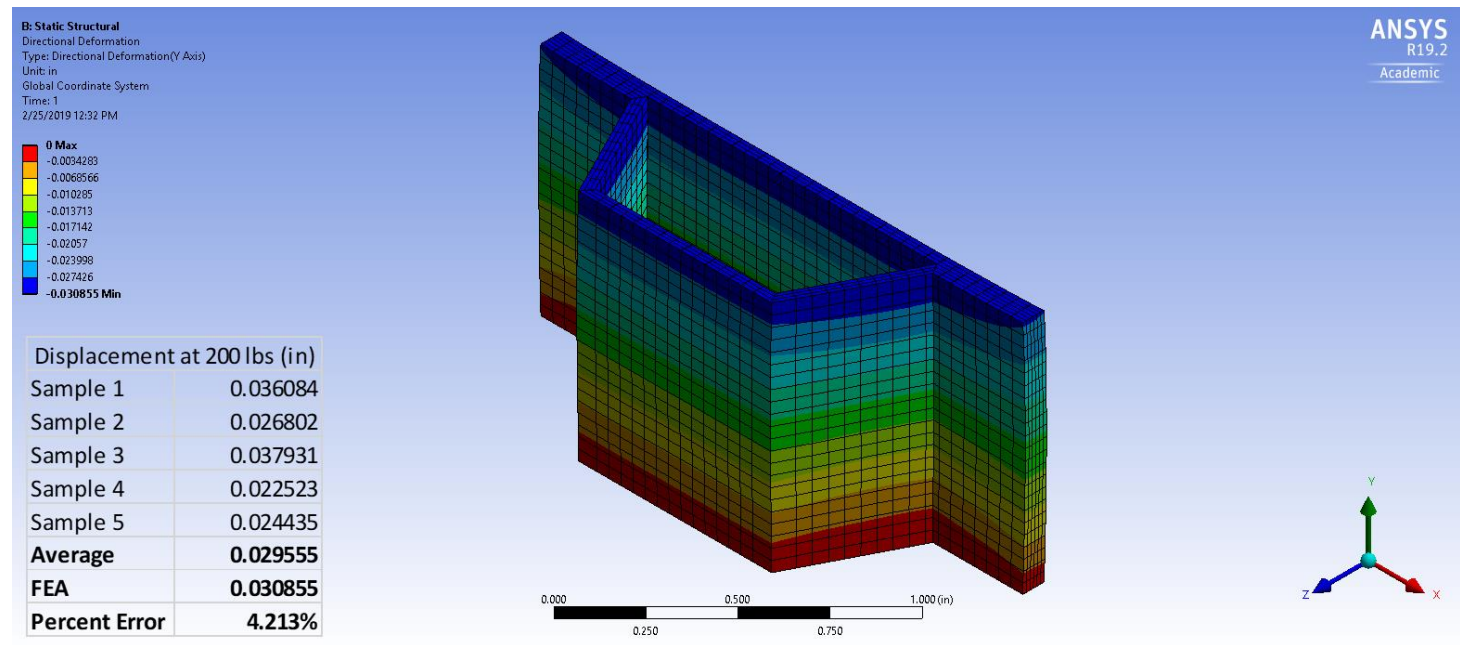

Figure 95: 1-Inch Hemp ANSYS and Experimental Results

The first sample analyzed was the 1-inch all-hemp sample, seen above in Fig. 95. As can be seen, with the static structural test and the tested and researched material properties for the hemp, the numerical displacement was 0.030855 inches, while the average displacement among the 5 samples at 200 pounds was 0.029555 inches. This resulted in a very low 4.213 percent error for the experimental compared to the numerical. While arriving at this low of an error percent is good with the mix of tested and researched material properties, it is important to note that a small difference in a single material 
property could change this deformation result greatly. Another source of error is the cardboard which was placed above and below the sample, causing the gradual increase of the load-displacement curve at the beginning. Despite this error, it is useful to know that the finite element analysis method is viable and should give ideal results with exact material properties and ideal failure behavior.

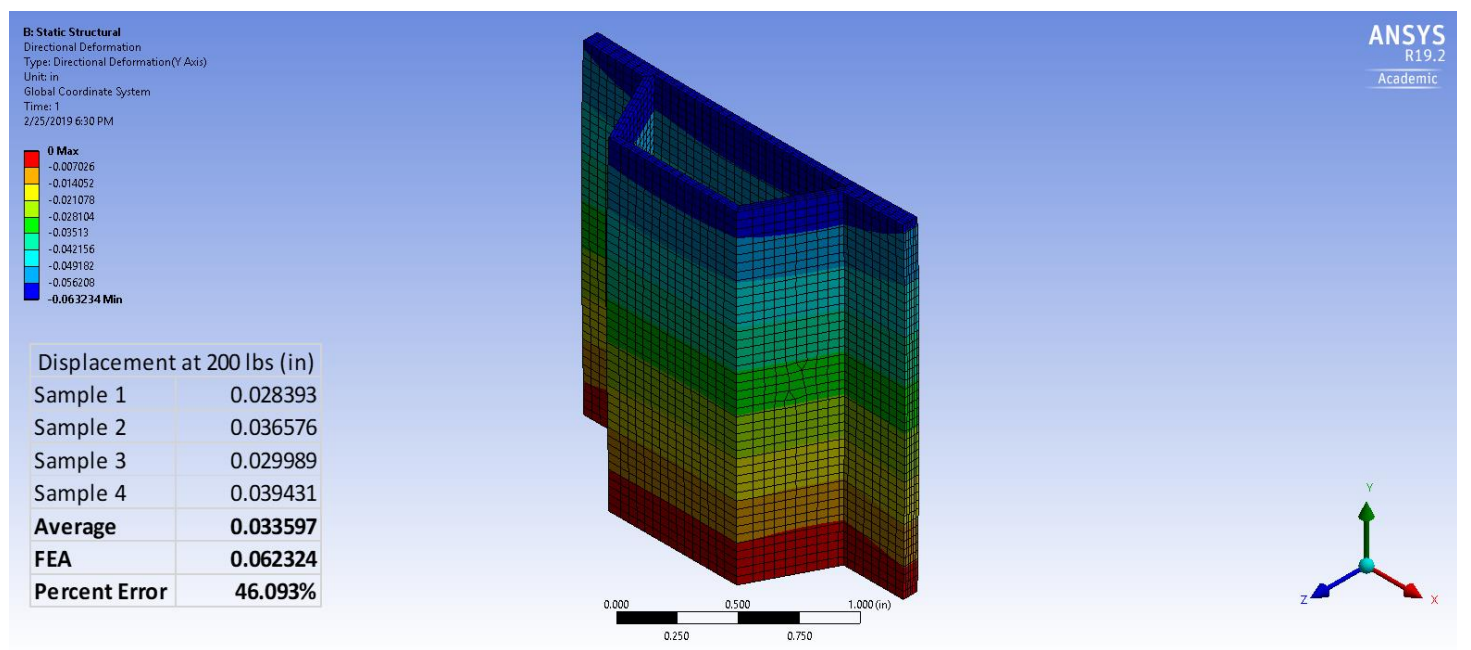

Figure 96: 2-Inch Hemp ANSYS and Experimental Results

Next, the model was altered, and the 2-inch sample was modeled in ANSYS, seen in Fig. 96. To make this alteration, the model was just extruded to two inches instead of one and the rest of the downstream steps were updated. It is important to make sure that displacement conditions and forces are assigned to the correct surfaces.

As the length doubles, it is expected that the vertical displacement would do the same as the overall stiffness should be halved. This two-inch sample should be like two one-inch samples stacked, in theory. This is much like the stiffness of two identical springs in series would be half of the stiffness of one of the springs. Despite this, the experimental 
displacement only increased slightly and, as discussed earlier, the stiffness of the sample only decreased slightly. This results in a much larger $46.1 \%$ error when compared to the finite element analysis results. However, the displacement and stiffness do have a correlation, even if it is slight.

Again, it is difficult to differentiate at what displacement and load the cardboard stops compressing and the sample starts to compress. For most cases, the data was graphed when the load increased past 10 pounds force, as this was determined to be enough to crush the cardboard and to start to compress the samples. However, some samples sat at a larger load around 50 pounds for a very long period of time, and data was graphed when this increased a noticeable amount. In an ideal study, test fixtures could be specially made for these samples, the samples would be perfectly levelled, and cardboard would not have been needed for grip and load distribution. Though, with the test fixtures and sander used, cardboard was the most viable solution after testing other buffer materials and methods. 


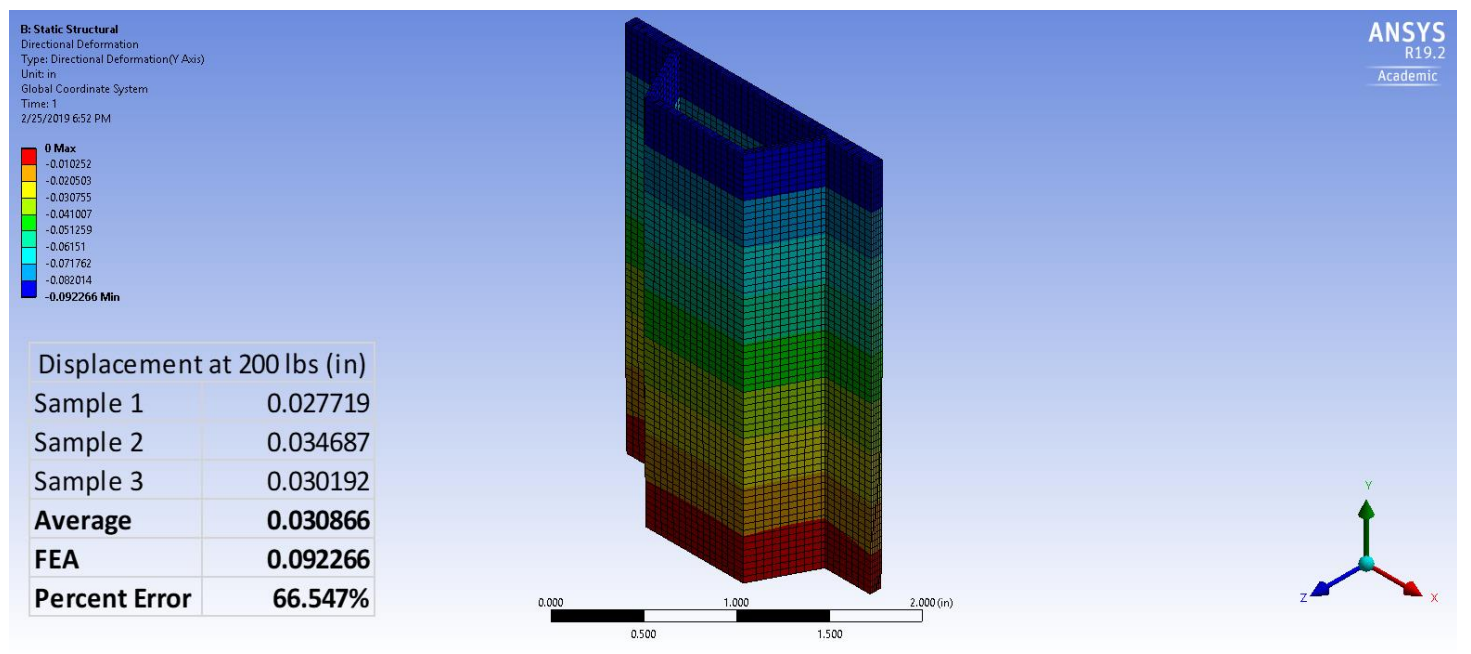

Figure 97: 3-Inch Hemp ANSYS and Experimental Results

The 3-inch hemp model had again larger percent error when comparing experimental data to the finite element data. As can be seen from Fig. 97, the finite element analysis put the compression displacement at around three times that of the 1-inch model, as expected. However, even at 200 pounds the experimental displacement stayed around the same as the previous two lengths. With the experimental displacement at 0.0309 inches - slightly lower than that of the 2-inch sample - and the numerical displacement at 0.0923 inches, the error increased by $20 \%$ to $66.547 \%$. While this unchanging displacement is likely affected by the cardboard buffer, despite attempts to mitigate this effect, it is also important to note the lack of significant stiffness change between the various all-hemp samples.

It was hypothesized that a linear extrapolation of the stiffness value - again derived from the slope of the linear section of the load-displacement curve - would provide another possible experimental displacement when extrapolated from the origin to 200 pounds force. This, hypothetically, would be the sample in an ideal compression test with no buckling, 
eccentric loading, uneven edges, or cardboard buffer. However, when extrapolated, the hemp samples showed much greater error overall and still had the issue of having similar stiffnesses, and therefore similar displacements at 200 pounds force.

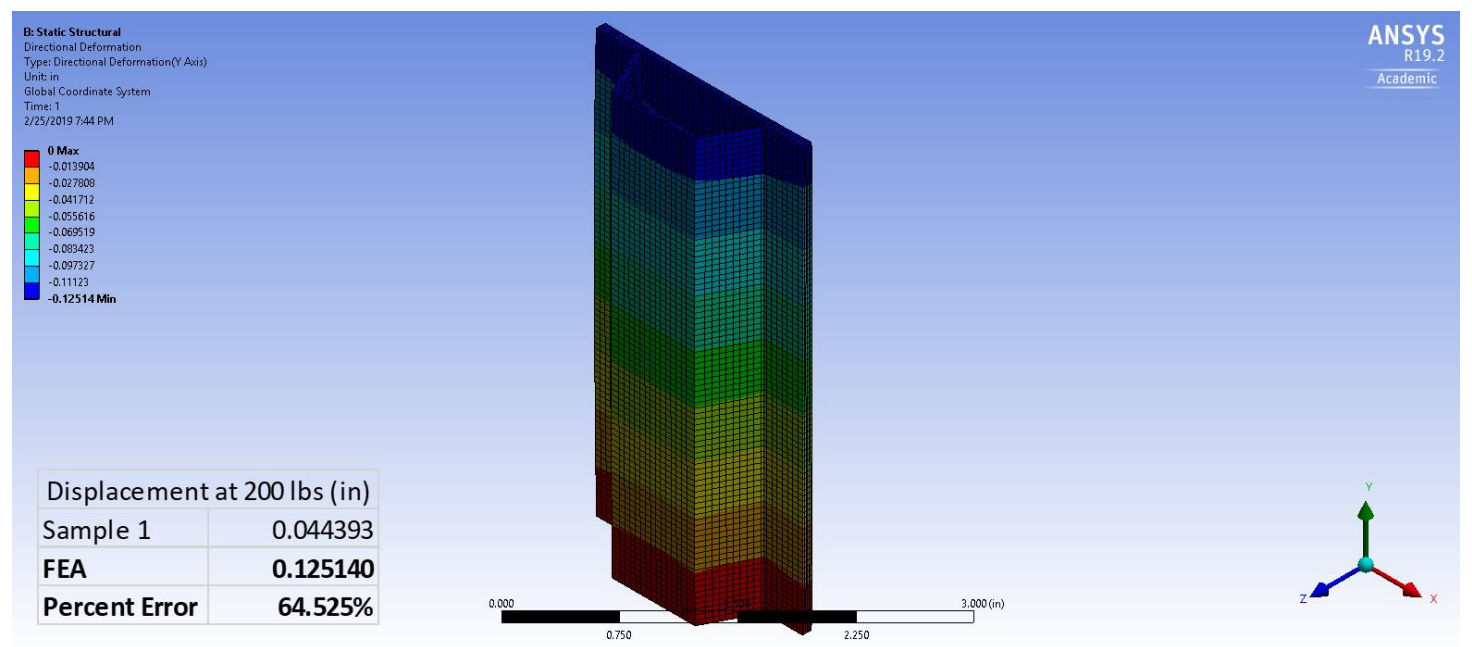

Figure 98: 4-Inch Hemp ANSYS and Experimental Results

The 4-inch hemp sample had a numerical 200 pounds force displacement value of 0.125 inches but an experimental displacement of 0.0444 inches. This resulted in a percent error of $64.525 \%$, slightly less than that of the 3 -inch samples. While this displacement was an increase from the previous three lengths, the percent error was still large. This was, curiously, while the stiffness again stayed very much the same. The main visual difference from this load-displacement cure to the previous load displacement curves was the much slower increase in slope from the origin.

Overall, results between all the different hemp finite element analysis had reasonable error margins when using somewhat mixed material properties and the static structural test. Because this was just the static structural test, the various strength- 
diminishing failure modes, like buckling, were not accounted for and are very difficult to simulate in finite element models, especially when dealing with complex composite materials and limited material properties. Because this was a static structural test, the results are largely based on cross-sectional area and material properties. This can be demonstrated by the 1-inch panel with the same cross-sectional area and hemp material properties seen in Fig. 99. This model, when placed under a 200 pound edgewise load, has a displacement of 0.03006 inches, almost identical to the 1-inch hemp corrugated sample.

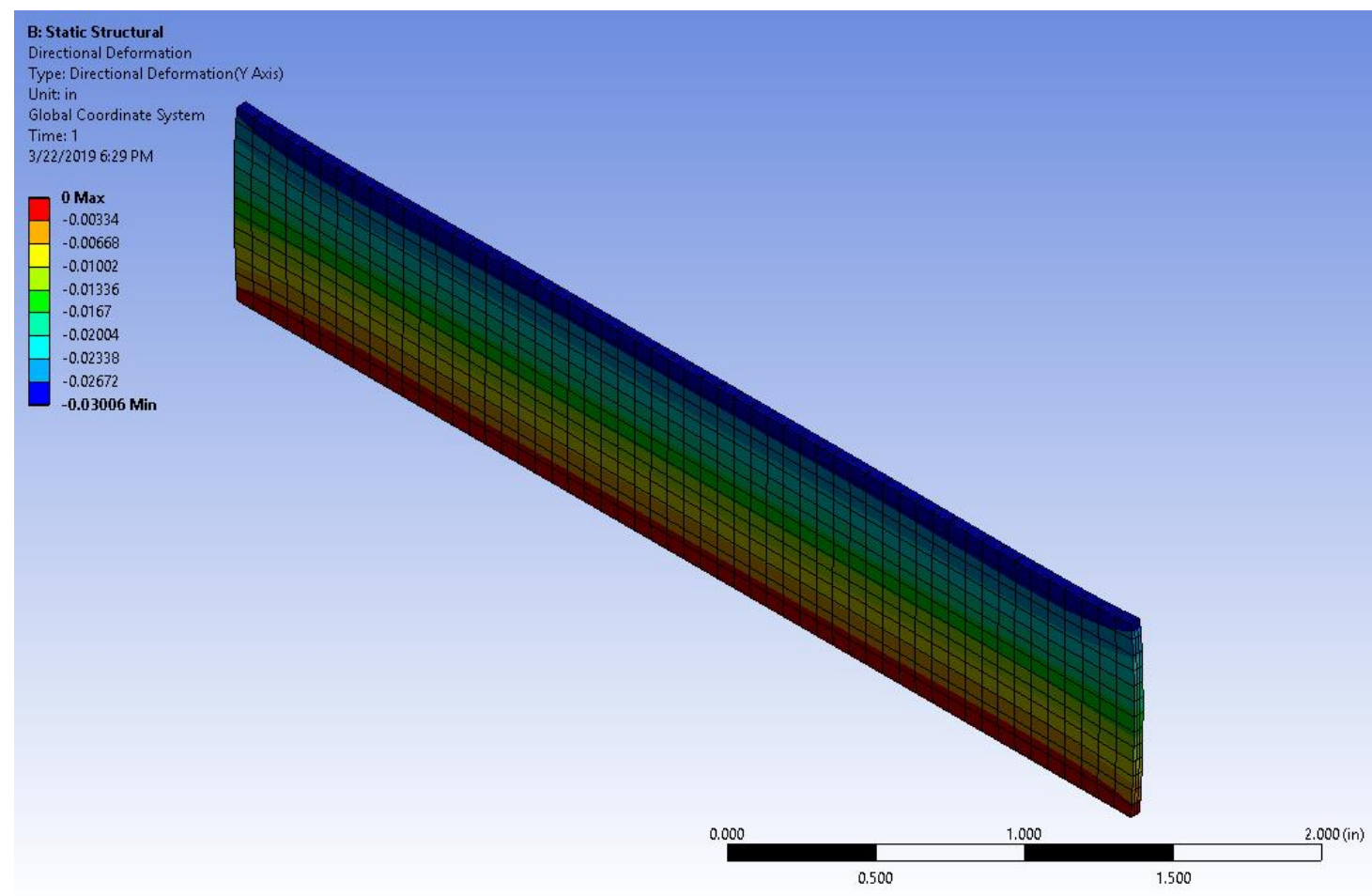

Figure 99: 1-Inch Hemp Panel ANSYS Results 


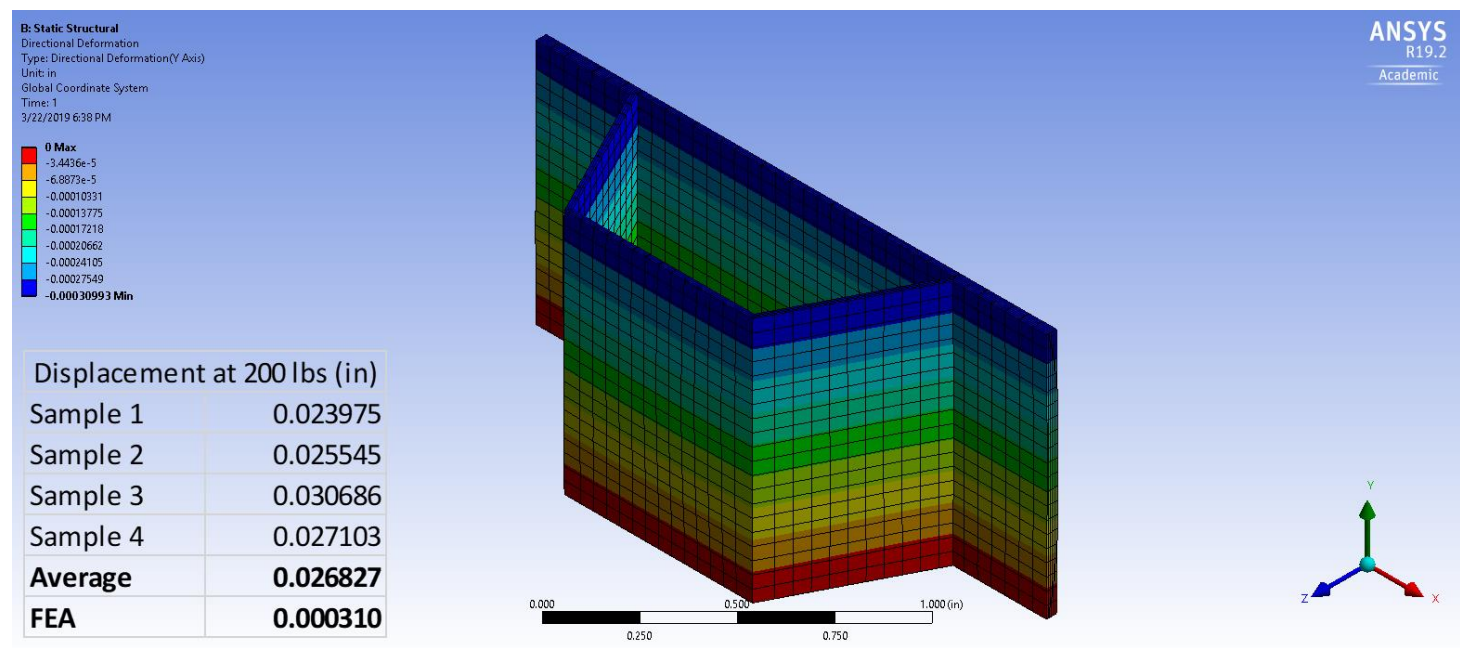

Figure 100: 1-Inch Carbon Fiber ANSYS and Experimental Results

Of the all-carbon fiber samples, the 1-inch sample was first to be modelled in ANSYS. Again, this model was set up using the ANSYS Composites Pre procedure discussed earlier. However, the material properties for the carbon fiber sample were researched and a fabric from the Composites Material Handbook, Volume 2, with similar weave, density, and ply thickness was used. These material properties, listed earlier, led to a displacement of 0.000310 inches at a 200 pounds load after the static structural test was done, as seen above in Fig. 100. This was very far from the experimental average of 0.026827 inches, which was much closer to the performance of the all-hemp samples. 
This difference can be explained qualitatively by the various failure modes that ANSYS does not account for in the static structural test and the thinness and brittleness of the carbon fiber samples. These samples measured 0.018 inches for three plies, less than half the thickness of the hemp laminate. This, combined with the brittleness of the carbon fiber, led to bearing stress failure at the edges which diminished the stiffness of the carbon fiber samples from the very start, even before early failure occurred due to bond failure. This highlights one of the main weaknesses of using finite element analysis to simulate thin, brittle structures. The ideal static structural test would be evaluating only the compressive strength of the structure and material, with the material undergoing no other failure modes. This would essentially be applying a force to an ideal column of the material. Reality, however, is much different with various bearing stress, buckling, and debonding failures. The goal of combining the hemp and carbon would be to stay as close to the ideal as possible for as long as possible during the compression test.

In addition, the material properties may not be quite right, since the values used come from a documented similar material. This, however, would not cause this large of a percent difference and should be noted as an additional, but minor, source of error. 


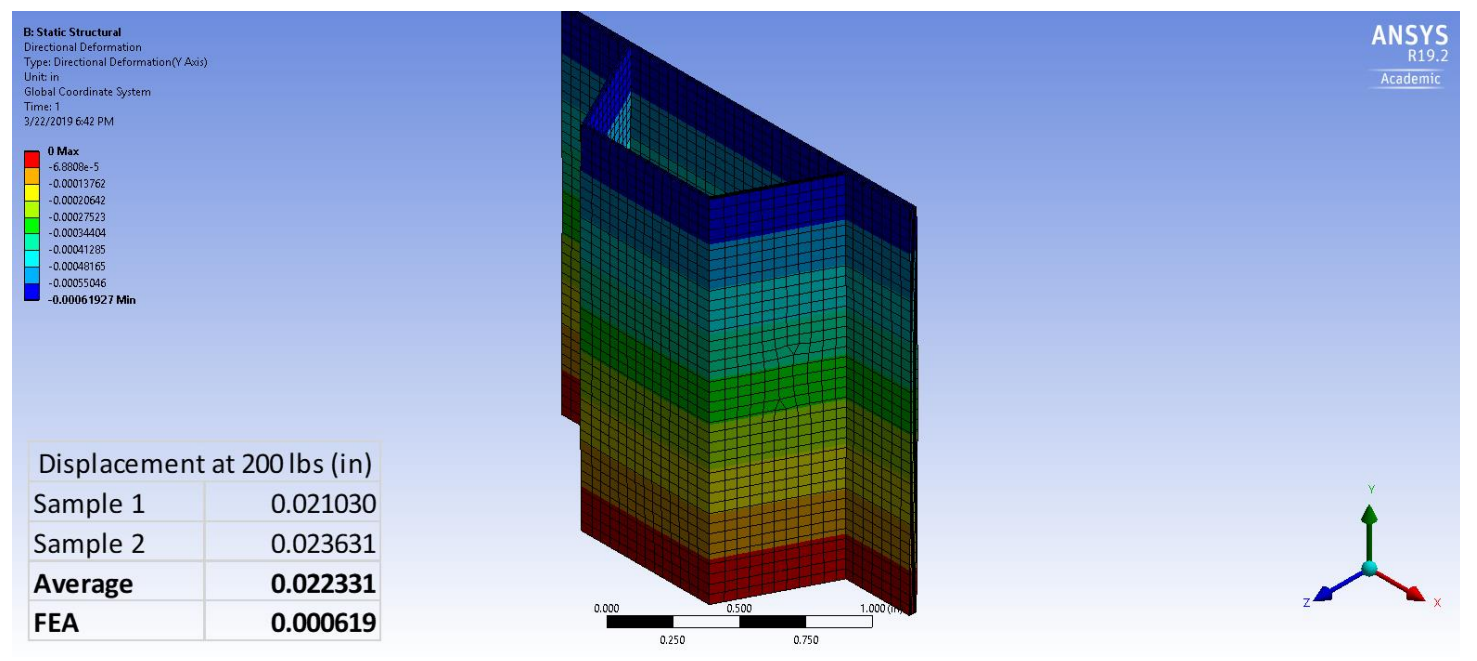

Figure 101: 2-Inch Carbon Fiber ANSYS and Experimental Results

The 2-inch all-carbon fiber sample had a similar outcome when comparing experimental and finite element analysis results. The 2-inch samples had a significantly lower experimental displacement of 0.022331 inches to go with a significantly higher stiffness of 48072 pounds per inch. This was compared to the experimental displacement and stiffness of 0.026827 inches and 28810 pounds per inch of the 1-inch samples. However, this still did not come close to the numerical displacement of 0.000619 inches, seen in Fig. 101. This is likely due to the same reasons listed above for the 1 -inch samples. There is a difference though - the 1-inch samples had consistent debonding between the skin and the core, causing the much more diminished mechanical properties, while the 2inch samples had low amounts of debonding due to the greater bonding surface area. This likely caused the great gain in mechanical properties from one inch to two inches in length. 


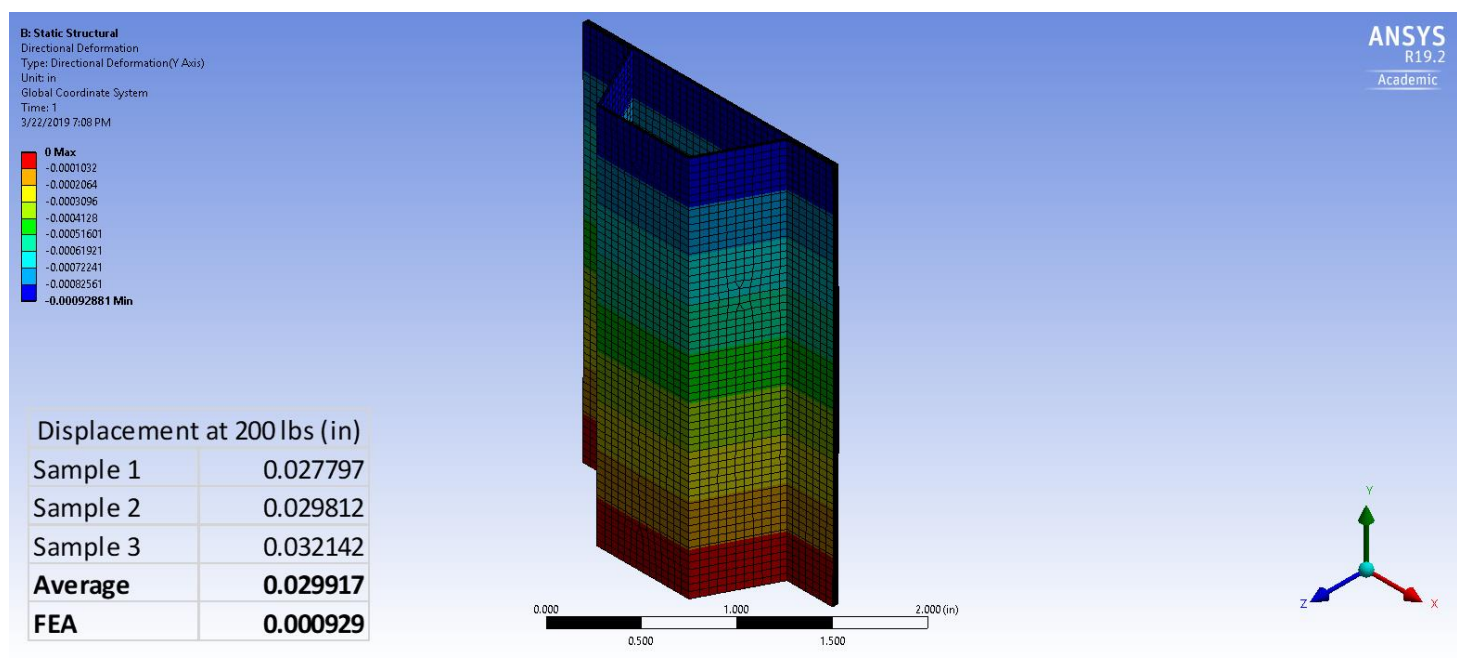

Figure 102: 3-Inch Carbon Fiber ANSYS and Experimental Results

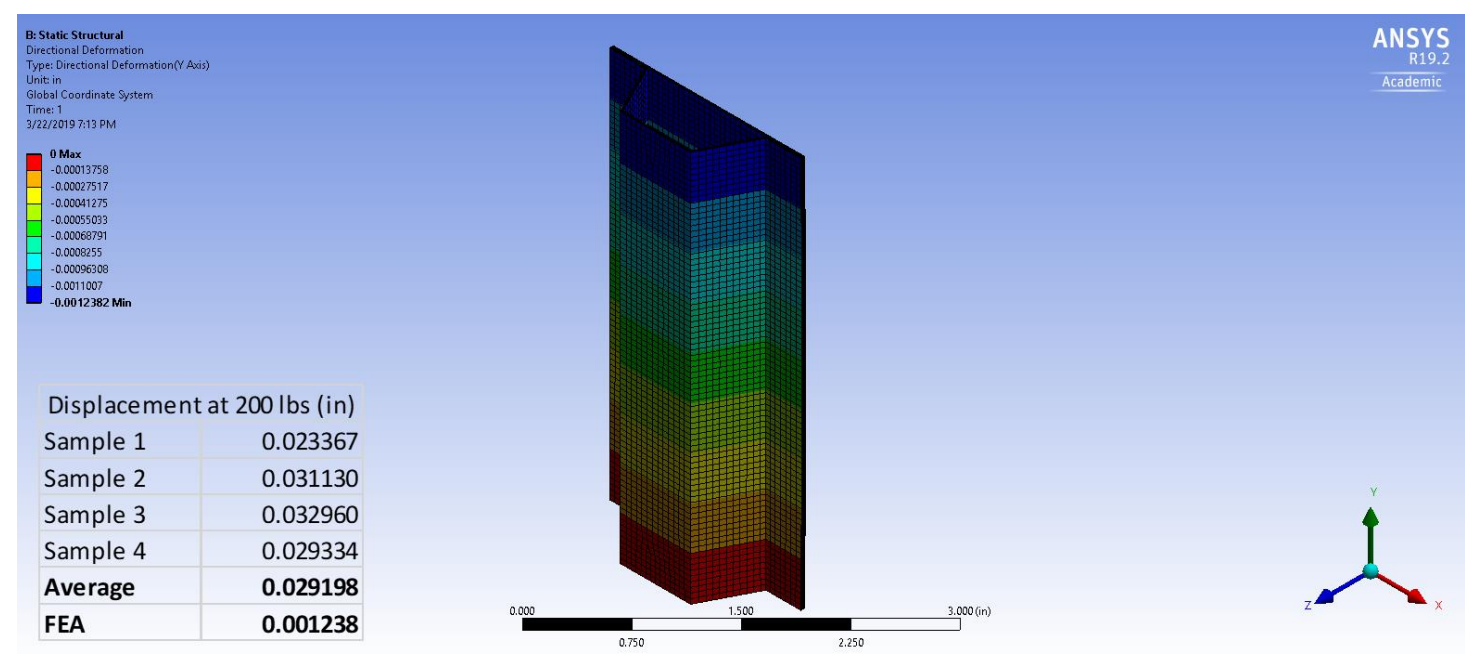

Figure 103: 4-Inch Carbon Fiber ANSYS and Experimental Results

The 3- and 4-inch carbon fiber models followed the same, almost linear trend when a 200-pound force was applied on the top surface, ending up with displacements of 0.000929 and 0.001238 inches respectively. These can be seen in Fig. 102 and 103. Again, the experimental displacements were very far from the ANSYS results due to the failure modes listed above and new failures introduced by the additional length - namely the global buckling and end rolling behaviors. It is important to note, however, that the 
displacement trend of the all-carbon fiber samples again follow the stiffness trend as did that of the all-hemp samples. Not only this, but the all-carbon fiber samples somewhat followed a decreasing stiffness and increasing displacement trend as length increased, not including the 1-inch sample with debonding.

This suggests that, if modeled correctly and with the proper boundary conditions, failure modes, and material properties, the carbon fiber, hemp, and hybrid corrugated structures can be modeled in finite element analysis. This, however, is very difficult. Not only is it difficult to model the various buckling, bearing stress, and end rolling failure modes with an isotropic material like steel, but to model these with an orthotropic, multiply, multi-material composite asymmetric structure is far outside the scope of this testing focused thesis. While the finite element analysis used is relevant for the hemp samples, which are more ductile, thicker, and behave somewhat like an isotropic material in compression, the static structural test is far too simplified for the complex behavior seen during the all-carbon fiber tests.

Eigenvalue buckling was attempted with the well-established carbon fiber material properties built in to ANSYS and, many of the local buckling failure modes seen in the finite element solution were also seen in experimental results, albeit with different numerical solutions. A very good example of this is seen in Fig. 103 and 104. In the first figure, the third buckling mode for the 1-inch carbon fiber finite element solution is shown, which is nearly identical to the experimental result one of the 1-inch hemp samples, shown in the Fig. 104. 


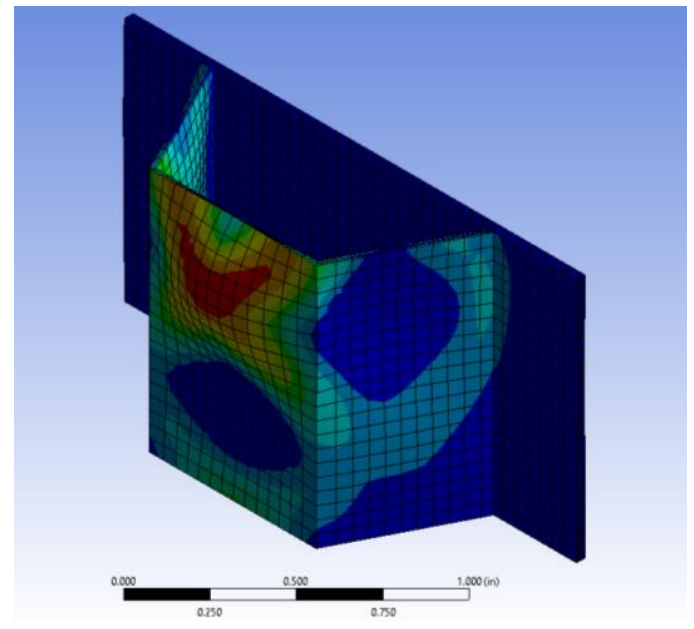

Figure 104: 1-Inch Carbon Fiber ANSYS Buckling Mode 3

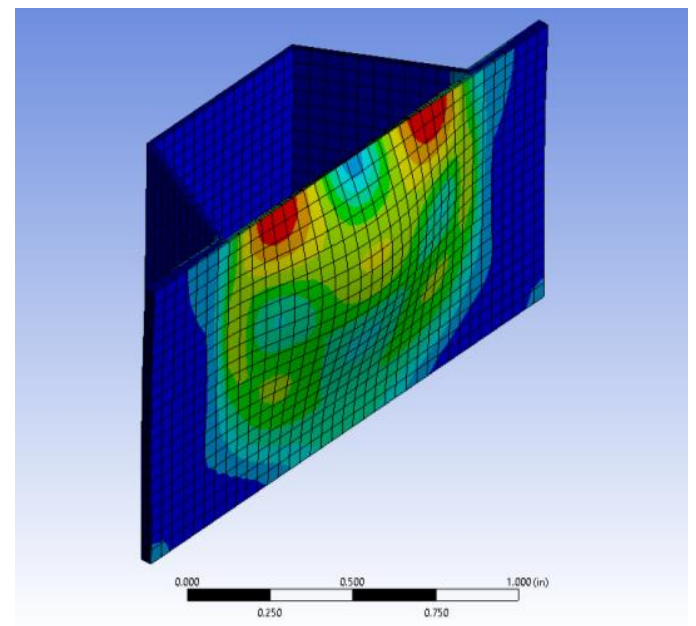

Figure 106: 1-Inch Carbon Fiber ANSYS Buckling Mode 1

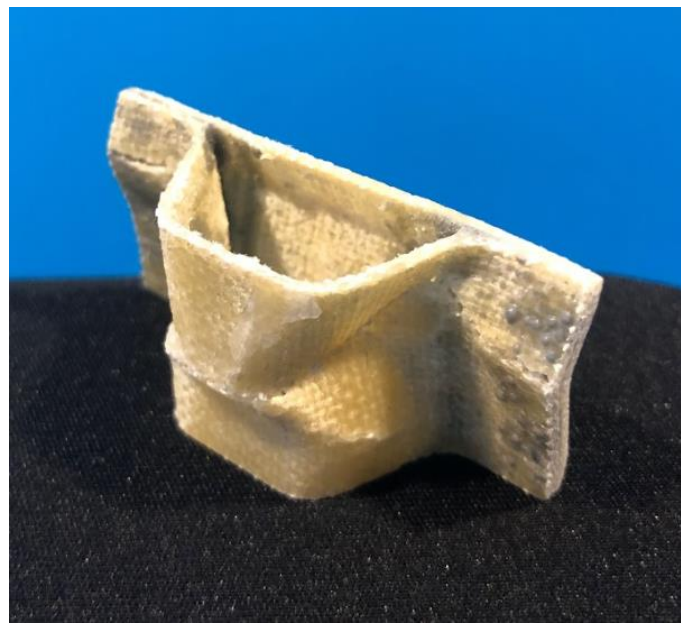

Figure 105: 1-Inch Hemp Experimental Local Buckling Results

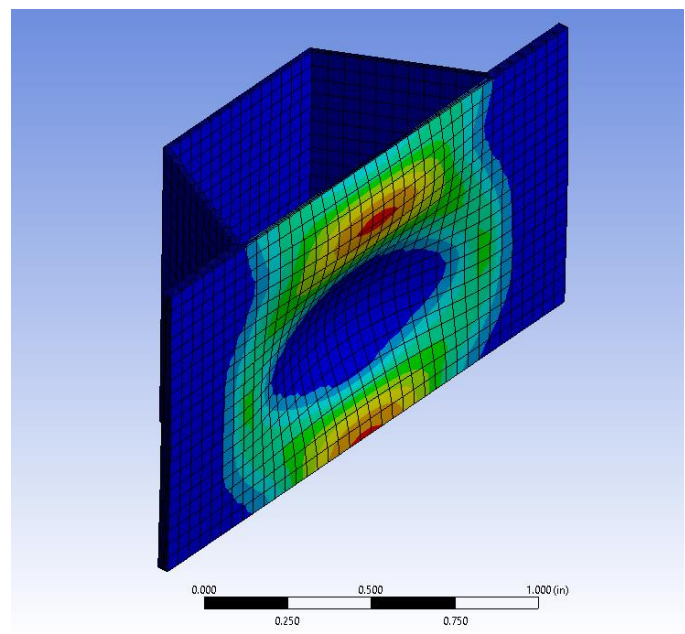

Figure 107: 1-Inch Carbon Fiber ANSYS Buckling Mode 2

Buckling modes 1 and 2, seen in Fig. 105 and 106 show the propensity of the skin to buckle. This causes the debonding failure mode that was discussed earlier and could be why, in addition to bearing stress, the carbon fiber samples never encountered local buckling. These lower buckling modes occur at lower load multipliers. The same can be said for the 2-inch samples, for which buckling mode 6 provided the corresponding local buckling mode to the experimental results, seen below in Fig. 107 and 108. 


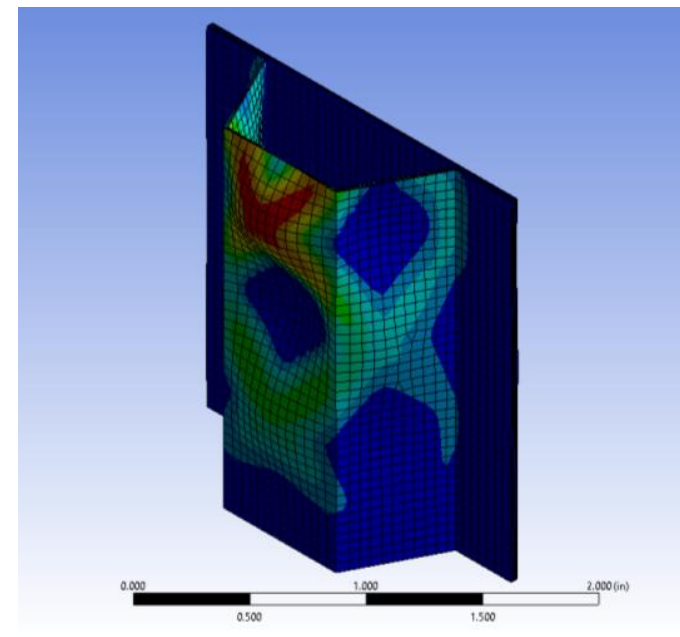

Figure 108: 2-Inch Carbon Fiber ANSYS Buckling Mode 6

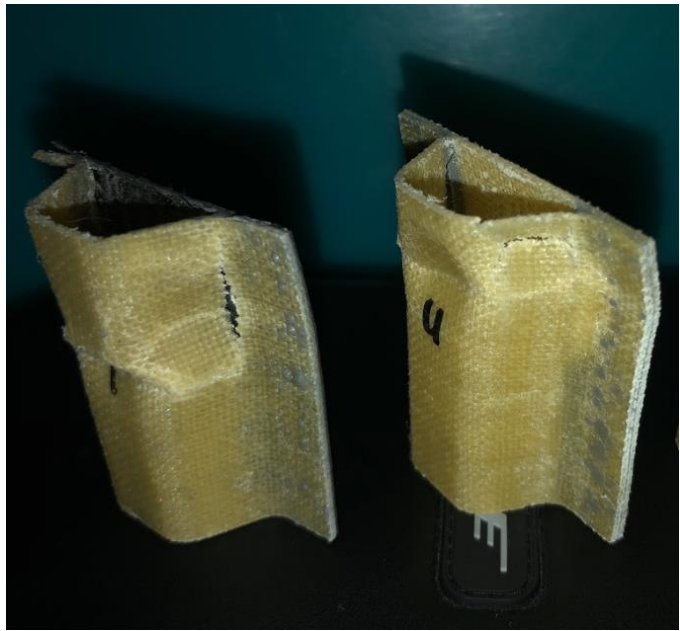

Figure 109: 2-Inch Hemp Core-Carbon Skin and All-Hemp Samples with Local Buckling

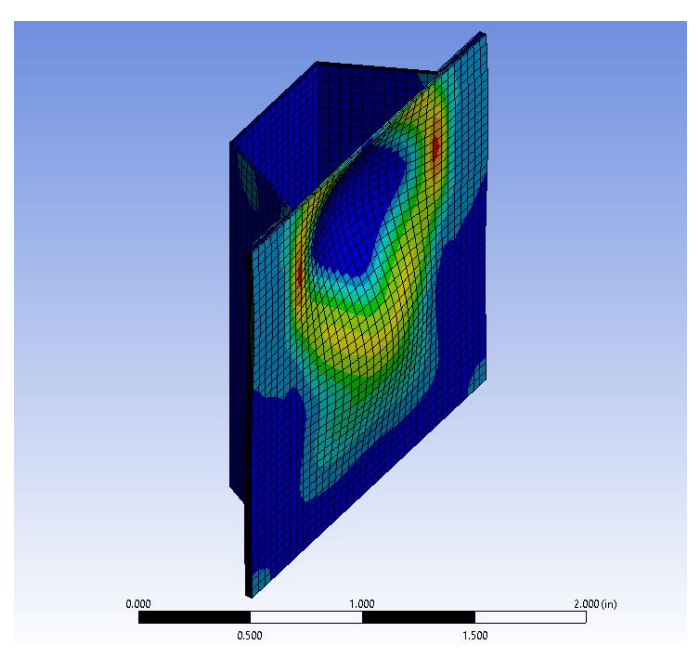

Figure 110: 2-Inch Carbon Fiber ANSYS Buckling Mode 1

could be a partial cause of the global

buckling failure. Buckling in this way, along with the flanges buckling, would cause global buckling at that location. This was identical with the 3- and 4-inch finite element results, where some buckling mode occurred at the location where the actual sample experienced global buckling. 


\section{CONCLUSIONS AND FURTHER RESEARCH}

Overall, the integrated hemp composite corrugated sandwich panel samples had mechanical properties much closer to the all-carbon fiber samples than the all-hemp samples, even outperforming the carbon fiber samples in some cases. In these cases, the equal stiffnesses of the carbon fiber skin and core, combined with local buckling, caused debonding between the core and skin. This prevented the carbon fiber samples from bearing their full load. Meanwhile, the mixed samples were able to bear their full load due to the different, but mutually beneficial, material properties of the hemp and carbon fiber.

Looking just at the 1-inch samples, where global buckling was minimally seen, the all-carbon fiber samples were outperformed in ultimate load by all the other samples. The all-carbon fiber samples had an average ultimate load of 1114 pounds with a standard deviation of 160.92 pounds. On average, the hemp core-carbon skin sustained a 30\% higher ultimate load than the all-carbon fiber samples with a standard deviation of 42.55 pounds, the lowest of the 1-inch samples. The all-hemp fiber samples sustained a $21 \%$ higher ultimate load with a standard deviation of 82.43 pounds. Lastly, the carbon core-hemp fiber had a 7\% higher ultimate load than that of the all-carbon fiber samples but had the highest deviation between samples with a standard deviation of 191.73 pounds.

The average stiffness of the 1-inch samples followed a similar trend. While the allhemp samples expectedly had the lowest stiffness of 28002 , the all-carbon fiber samples were very close with a stiffness of 28810 pounds per inch. This is because of the multiple failure modes of the load-displacement curve, likely due to the splintering and debonding of the sample skins and cores. The average stiffness of the hemp core-carbon skin samples 
was the highest $-72 \%$ higher than that of the all-carbon fiber samples at 49510 pounds per inch. The ability of the hemp to adhere to the carbon fiber allowed the corrugated sandwich structure to leverage the stiffness of the carbon fiber without debonding into two separate and weaker structures. The stiffness of the carbon core-hemp skin samples was $6 \%$ higher than that of the all-carbon fiber samples at 30664 pounds per inch. While the hemp corecarbon skin samples had the highest ultimate load, the margin by which they had the highest stiffness was drastic.

For the 2-inch samples, the all-carbon fiber samples had the highest average ultimate load and stiffness of 1504 pounds and 48072 pounds per inch while the all-hemp samples had the lowest of each at 1233 pounds and 21019 pounds per inch. The carbon core-hemp skin and hemp core-carbon skin samples, meanwhile, had average ultimate loads of 1293 and 1274, slightly higher than that of the all-hemp samples. Their stiffnesses were more towards that of the all-carbon fiber samples though, at 38528 and 35868 pounds per inch respectively. At this length, although the samples aren't long enough to become significantly unstable, asymmetric cross-section and the hemp having a lower stiffness than the carbon fiber causes some global buckling to occur in some portions of the mixed sample tests. This diminished the ultimate load and stiffness. Meanwhile, the all-carbon fiber sample was stiff throughout, not buckling and having enough bonding surface area to remain intact and hold some of the highest mechanical properties seen in all configurations. Moving from the 2-inch samples, the 3- and 4-inch samples for each configuration had less definitive results, likely stemming from the widely varied failure modes occurring in the tests of these lengths. These failure modes and varying levels of impact on the results of these tests and the mechanical properties of each configuration were diminished to various 
degrees as a result. While this did not invalidate the results, they were indeed found to be less conclusive due to the various dimensions that the many different failure modes added. This made comparative analysis between these lengths and the shorter lengths much less relevant. Not only did comparing the results of these tests become difficult due to the type of failure being different, but they quickly were shown to be very difficult to model in finite element analysis.

To conclude, while the all-carbon fiber samples showed high mechanical properties at 2, 3, and 4 inches, the 1 -inch samples had very early failure due to the debonding of skin from core. This was consistent with previous research and was a severe disadvantage as the structure separates into multiple pieces, losing the advantages of a sandwich panel. This was remedied by using a hemp core, allowing the sample to reach its full ultimate edgewise compressive strength. Although the mechanical properties dropped as length was increased, this was thought to be a result of an asymmetric cross-section causing buckling behaviors - an expected yet underestimated consequence. Despite this, the hemp fibers showed promise having usage in hybrid composite structures. This was due not only to their comparable strength but also their ability to reach a high ultimate load and fail gradually, rather than drastically. Replacing portions of traditional composites with hemp fibers, as discussed, also has a myriad of benefits, from being more ecologically friendly and sustainable to being much less costly. These hybrid composite structures could replace secondary structures in the construction, automotive, and aerospace industries, though additional testing would need to be done to determine the ratio of hemp fiber to carbon fiber, temperature effects, and moisture absorption. 
Such additional testing should also include a narrowing of focus down to a single dimension, or single mode of failure. This would mean not only focusing just on global buckling or using a symmetric structure to focus just on axial compression, but also increasing resolution and exploring and optimizing layup technique, cure cycle, and bonding adhesive. Layup technique could be explored using pre-impregnated composites, debulk cycles, and number of plies, as this research focused on 3-ply wet layups. Cure cycle could also be optimized, as this research used a room-temperature vacuum layup, although it was beneficial to use the same epoxy system for both the hemp and carbon fiber. The bonding adhesive is a very important factor and could be explored using different adhesives, either focusing on axial strength or shear strength. The Magnolia epoxy used in this research was very strong in both, and the strongest that was on hand, but adhesives used in the proposed application of these structures would need to be tested. 


\section{REFERENCES}

[1] Liu, Tao. "Dynamic compressive response of wrapped carbon fibre composite corrugated cores." Elsevier (n.d.): n. pag. Web. 24 Jan. 2017.

[2] Abdewi, Elfetori F., S. Sulaiman, A.M.S. Hamouda, and E. Mahdi. "Effect pf geometry on the crushing behaviour of laminated corrugated composite tubes." Journal of Materials Processing Technology (2005): n. pag. Web. 25 Jan. 2017.

[3] Thill, Cristophe. "Investigation of trapezoidal corrugated aramid/epoxy laminates under large tensile displacements transverse to the corrugation direction." Composites (n.d.): n. pag. Web. 27 Jan. 2017.

[4] Lurie, S.a., Yu.o. Solyaev, D.b. Volkov-Bogorodskiy, V.m. Bouznik, and A.a. Koshurina. "Design of the corrugated-core sandwich panel for the arctic rescue vehicle." Composite Structures 160 (2017): 1007-019. Web. 29 Jan. 2017.

[5] Han, Bin, Keke Qin, Bo Yu, Bo Wang, Qiancheng Zhang, and Tian Jian Lu. "Honeycomb-corrugation hybrid as a novel sandwich core for significantly enhanced compressive performance." Materials \& Design 93 (2016): 271-82. Web. 8 Feb. 2017. 
[6] Shaw, A.d., I. Dayyani, and M.i. Friswell. "Optimisation of composite corrugated skins for buckling in morphing aircraft." Composite Structures 119 (2015): 227-37. Web. 13 Apr. 2017.

[7] Winkler, M., and G. Kress. "Influence of corrugation geometry on the substitute stiffness matrix of corrugated laminates." Composite Structures 94 (2012) 28272833 (2012): n. pag. Web. 10 Feb. 2017.

[8] He, Wentao, Jingxi Liu, Bo Tao, De Xie, Jiayi Liu, and Min Zhang. "Experimental and numerical research on the low velocity impact behavior of hybrid corrugated core sandwich structures." Composite Structures 158 (2016): 30-43. Web. 1 Apr 2017.

[9] Dhakal, H.n., and Z. Zhang. "The Use of Hemp Fibres as Reinforcements in Composites." Biofiber Reinforcements in Composite Materials, 2015, pp. 86-103., doi:10.1533/9781782421276.1.86. Web. 4 June 2018.

[10] “Nonwood Bio-Based Materials.” Performance of Bio-Based Building Materials, 2017, pp. 97-186., doi:10.1016/b978-0-08-100982-6.00003-3. Web. 4 Feb 2019. 
[11] Tawfik, Basem E., et al. "Weight Reduction and Strengthening of Marine Hatch Covers by Using Composite Materials." International Journal of Naval Architecture and Ocean Engineering, vol. 9, no. 2, 2017, pp. 185-198., doi:10.1016/j.ijnaoe.2016.09.005.

[12] Canadian Hemp Trade Alliance » Products » CHTA, www.hemptrade.ca/eguide/fibre-production/fibre-processing.

[13] Christian, Sarah, and Sarah Billington. "Sustainable Biocomposites for Construction.” COMPOSITES \& POLYCON 2009, 15 Jan. 2009.

[14] Bhat, G., and H. Rong. "Biodegradable Nonwovens." Biodegradable and Sustainable Fibres, 2005, pp. 310-342., doi:10.1533/9781845690991.310.

[15] “HempTraders.com.” Hemp Traders: Suppliers of Fine Hemp Products, www.hemptraders.com/Hemp-Fiber-s/1828.htm.

[16] "Industrial Hemp." How Products Are Made, www.madehow.com/Volume6/Industrial-Hemp.html.

[17] "Honeycomb Panels, Honeycomb Cores." Plascore, www.plascore.com/honeycomb/honeycomb-panels/. 
[18] Sweeney, Benjamin A. "The Effect of Biocomposite Material In A Composite Structure Under Compression Loading.” Feb. 2017.

[19] Miles, David. "History of Corrugated Iron." World Archaeology, no. 28, 7 Mar. 2008.

[20] “Corrugated Iron Supplies Melbourne | Corrugated Iron 'ROLEX' Roofing.” Robot Building Supplies, robotbuildingsupplies.com.au/product/corrugated-iron-rolexcolour-steel-roofing/.

[21] Hou, Shujuan, et al. "Crashworthiness Optimization of Corrugated Sandwich Panels.” Materials \& Design, vol. 51, 2013, pp. 1071-1084., doi:10.1016/j.matdes.2013.04.086.

[22] Tawfik, Basem E., et al. "Weight Reduction and Strengthening of Marine Hatch Covers by Using Composite Materials." International Journal of Naval Architecture and Ocean Engineering, vol. 9, no. 2, 2017, pp. 185-198., doi:10.1016/j.ijnaoe.2016.09.005.

[23] Das, Sujit. "Life Cycle Assessment of Carbon Fiber-Reinforced Polymer Composites." The International Journal of Life Cycle Assessment, vol. 16, no. 3, 2011, pp. 268-282., doi:10.1007/s11367-011-0264-z. 